\title{
Processing referential expressions in German Sign Language
}

\author{
Dissertation \\ zur Erlangung des philosophischen Doktorgrades \\ an der Philosophischen Fakultät der Georg-August-Universität Göttingen
}

vorgelegt von

Anne Wienholz

aus Schwedt/Oder

Göttingen 2018 



\section{Acknowledgments}

The research presented in this dissertation was funded by the Deutsche Forschungsgemeinschaft (DFG, German Research Foundation) - Priority Program (SPP 1727) Xprag.de: New Pragmatic Theories based on Experimental Evidence, research project Discourse Referents in Space - Anaphora Resolution in German Sign Language.

First and foremost, I want to thank my supervisors Prof. Dr. Nivedita Mani and Prof. Dr. Markus Steinbach for giving me the opportunity to conduct this research even if I had no clue about sign language linguistics and did not know any sign language in the beginning. This already shows that both are always open to strike new paths without knowing where this path leads to, which is essential when you enter new research areas. I could not imagine a better combination of supervisors: with Markus being an expert of sign language linguistics and with Nivi as an expert of experimental psycholinguistic research, I had the best support for developing myself as a professional researcher and a private person. I am enormously grateful that I had them by my side all the time.

In addition, I am exceedingly grateful to be part of two research groups, which always allowed me to look at my research from various angles. On the one hand, there are the current and former members of the SignTeam at the University of Goettingen. A special thank you goes to Annika Herrmann for always finding time to discuss my questions and motivating me to keep going. For fruitful discussions on various topics, I want to thank the rest of the SignTeam: Cornelia Loos, Elisabeth Volk, Jana Hosemann, Nina-Kristin Pendzich, Sina Proske, Sukie Brinkmann, Tanja Recke, Thomas Finkbeiner, but most of all Jens-Michael Cramer and Liona Paulus for rescuing me from getting lost in the jungle of German Sign 
Language grammar and vocabularies. Additionally, thank you to my colleagues at the PsychLab Heinke Syring, Katja Suckow and Thomas Weskott for their support in different situations. On the other hand, there are my colleagues at the WortSchatzInsel at the Psychology of Language research group. Being part of your team and your journal club meetings enabled me to learn a lot about different topics that, otherwise, I would not have thought about. Therefore, thank you to: Daniela Ávila, Julia Brase, Lena Ackermann, Melanie Schreiner, Sarah Eiteljörge and Vivien Radtke.

Furthermore, many thanks also to Konstantin Grin for introducing me to the secrets of German Sign Language during our weekend-language-course-sessions. Moreover, thank you to Konstantin, Danny Canal and Jens-Michael for being that patient when we had to record millions of stimulus sentences (at least it felt like a million) for all experiments. Additionally, I am glad about the support of various student assistants in recording and cutting all the videos. A special thank you to Alexander Syring for his excellent technical support at any time. Without all of you, it would not have been possible to run the studies.

Fortunately, I was able to present my work at international conferences, which offered the possibility to get feedback by internally renowned researchers. These conference and workshop visits were supported by the Leibniz ScienceCampus, the Research Training Group 2070 Understanding Social Relations and the Graduate School of Humanities Göttingen.

However, the whole way leading to this dissertation would have been much harder and less fun without my office mate and friend Derya Nuhbalaoglu. We both started our dissertation-adventure together without knowing how to manage all the new things that we were encountered with. Together, we discovered the German culture, including Christmas cookies and Federweißer, and we traveled to many parts of the world where we learned about 
crazy Bavarian kings, met Australian wild animals and enjoyed the most relaxing Icelandic bath. Our Humboldtallee-squad with Simone Gerle always took care of enough breaks filled with coffee, cake and conversations. Thank you for these moments!

Finally, I am exceedingly grateful for the love of my family as well as for their endorsement and support at any time. 



\begin{abstract}
In sign languages, physically absent discourse referents are assigned manually and/or nonmanually to referential locations on the horizontal plane of the signing space. These locations can be used to refer back to the associated referent in the subsequent discourse by establishing a referential relation between the referent and a referential expression that can take various forms. This dissertation examines the processing of referential expressions in German Sign Language and the factors influencing these processing mechanisms by using two different experimental techniques. Chapter 1 investigated the presence of a default pattern during the assignment of discourse referents to referential locations applying in the absence of overt localization cues. This pattern suggests that right-handed signers associate the first mentioned referent with the ipsilateral (right) while assigning the second referent to the contralateral (left) area of the signing space. Event-related potential (ERP) data provide evidence for the proposed pattern and supports the claim that signers assign distinct and contrastive referential locations to two different discourse referents. Additionally, ERP data presented in Chapter 2 suggest a first mention (subject) preference used to resolve the reference relations of pronominal pointing signs. Moreover, these data support the first mention (subject) preference to be a modalityindependent mechanism. In an eye tracking study using the Visual World Paradigm, Chapter 3 investigates whether overt localization of a discourse referent leads to facilitation during the processing of a co-referential expression, i.e., a bare noun in this case, in subsequent discourse. Results indicate that localization increases the prominence and hence the accessibility of a referent leading to easier processing of its co-referential expression, but only if this referent occurs in the subject position. This suggests that the effects of overt localization and a first mention (subject) preference interact during the processing of referential expressions. Since the eye tracking method in combination with the Visual World Paradigm was only used rarely in
\end{abstract}


previous studies in sign languages, Chapter 4 presents another eye tracking study testing the appropriateness of the experimental setting during the presentation of naturally signed sentences. Therefore, the study examines whether a phonological priming effect can be observed in natural sentences using this setting and whether effects differ based on the phonological relation of prime-target sign pairs embedded in the sentences. Results provide evidence that phonological parameters affect processing differently such that sign recognition is facilitated for signs sharing the same handshape parameter, but is slowed down when sharing the same location parameter. This study suggests that sub-lexical features influence sign recognition, but more importantly, it proves that this experimental setting can be used to investigate sign language processing.

\section{Zusammenfassung}

In Gebärdensprachen werden physisch abwesende Diskursreferenten referentiellen Bereichen in der horizontalen Eben des Gebärdenraums zugeordnet. Diese können dazu verwendet werden, um auf Referenten, die mit diesem Bereich assoziiert wurden, im nachfolgenden Diskurs zu verweisen. Somit wird eine referentielle Beziehung zwischen Referent und einem referentiellen Ausdruck, der viele Formen haben kann, hergestellt. Die vorliegende Dissertation untersucht mit Hilfe verschiedener experimenteller Methoden die Verarbeitung verschiedener Formen von referentiellen Ausdrücken in Deutscher Gebärdensprache und welche Faktoren die zugrundeliegenden Verarbeitungsmechanismen beeinflussen. Kapitel 1 erforscht die Verwendung einer Standardvorgehensweise bei der Zuordnung von Diskursreferenten zu referentiellen Bereichen, wenn keine offensichtliche Verortung stattfindet. Bei dieser Vorgehensweise assoziieren rechtshändige Signer (Personen, die mit Hilfe einer Gebärdensprache kommunizieren) zuerstgenannte Referenten mit der rechten (ipsilateralen) 
Seite des Gebärdenraums während sie zweitgenannte Referenten der linken (kontralateralen) Seite zuordnen. Die ereigniskorrelierten Potentiale (EKP) der Studie liefern Beweise für die vorgeschlagene Vorgehensweise und unterstützen die Annahme, dass Signer unterschiedliche und gegensätzliche referentielle Bereiche für die Verortung von Referenten verwenden. Außerdem zeigen die EKP-Daten in Kapitel 2, dass es eine Subjektpräferenz bei der Verarbeitung von pronominalen Zeigegebärden gibt, d.h. dass Pronomen als ko-referentiell mit einem vorangegangenen Subjekt interpretiert werden. Das lässt vermuten, dass die Subjektpräferenz ein modalitätsunabhängiger Mechanismus ist, da dieser auch in vielen Lautsprachen nachgewiesen wurde. In einer Eye Tracking-Studie unter der Verwendung des Visual World Paradigma untersucht Kapitel 3, ob die offensichtliche Verortung eines Diskursreferenten die Verarbeitung eines mit diesem ko-referentiellen Ausdrucks erleichtert. Die Ergebnisse weisen darauf hin, dass offensichtliche Verortung die Wichtigkeit und somit die Erreichbarkeit eines Referenten in der mentalen Repräsentation des aktuellen Diskurses erhöht und somit zu schnellerem Verstehen eines ko-referentiellen Ausdrucks führt. Das funktioniert allerdings nur, wenn der Referent vorher in der Subjektposition im Satz aufgetreten ist. Das lässt vermuten, dass die Einflüsse von Subjektpräferenz und offensichtlicher Verortung während der Verarbeitung ko-referentieller Ausdrücke interagieren. Da der in Kapitel 3 verwendete experimentelle Aufbau in früheren Studien zu Gebärdensprachen bisher nur selten und zur Untersuchung ganzer Sätze noch gar nicht verwendet wurde, präsentiert Kapitel 4 eine Studie, die die Eignung des Aufbaus für die Untersuchung natürlicher gebärdeter Sätze testet. Dafür erkundet die Studie, ob ein phonologischer Primingeffekt auf Satzebene mit Hilfe des beschriebenen experimentellen Aufbaus nachgewiesen werden kann. Außerdem wird untersucht, ob sich dieser Effekt unterscheidet basierend auf der Variation der phonologischen Zusammenhänge der getesteten Gebärdenpaare. Die Ergebnisse zeigen, dass die phonologischen Eigenschaften einer Gebärde die Verarbeitung dieser beeinflusst. Die Identifizierung einer Gebärde wird erleichtert, wenn Gebärden die gleiche Handform haben, 
aber die Erkennung wird erschwert bei Gebärden mit der gleichen Ausführungsstelle. Diese Studie zeigt, dass sub-lexikale Eigenschaften die Verarbeitung einer Gebärde beeinflussen. Doch viel wichtiger, die Studie beweist, dass der experimentelle Aufbau verwendet werden kann, um die Verarbeitung von Sätzen in Gebärdensprachen zu untersuchen. 


\section{Table of contents}

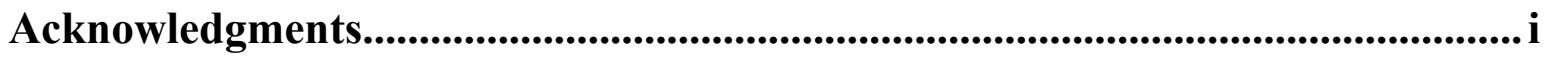

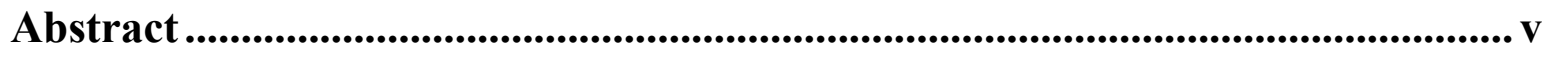

Zusammenfassung..................................................................................................vi

Table of contents............................................................................................................. ix

General introduction ............................................................................................................ 1

1 Pointing to the Right Side? An ERP Study on Anaphora Resolution in

German Sign Language ........................................................................... 13

1.1 Introduction ................................................................................................ 14

1.1.1 Discourse referents and referential locations ..................................... 15

1.1.2 Localizing discourse referents in signing space ................................... 16

1.1.3 Antecedent reactivation in spoken and sign languages .......................... 17

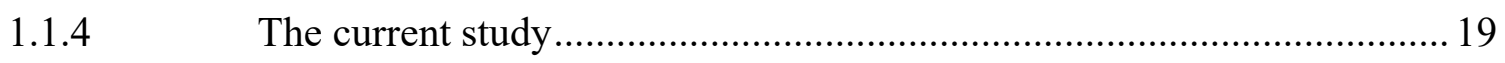

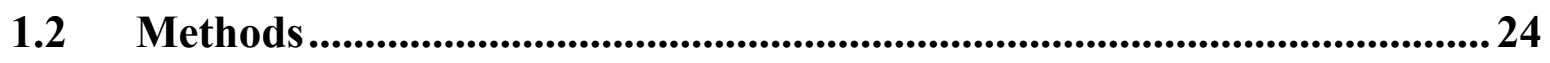

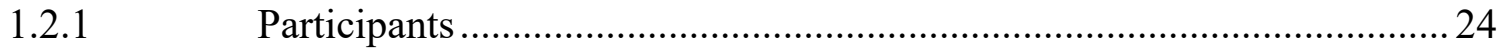

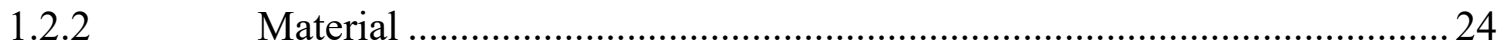

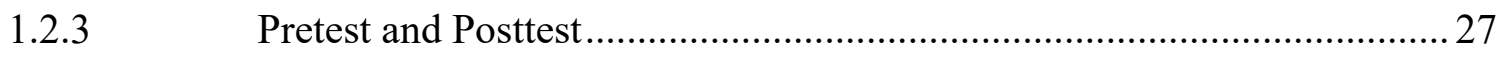

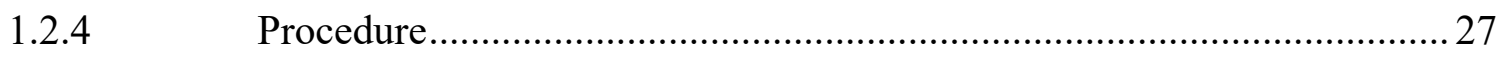

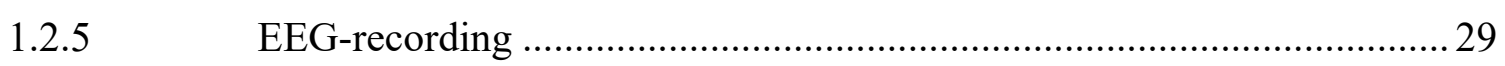

1.3 Results ........................................................................................................... 31

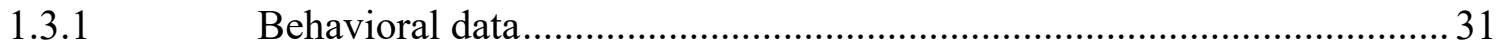

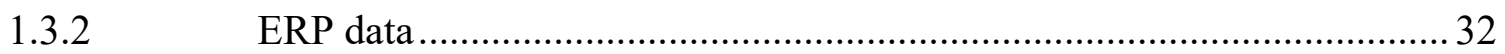

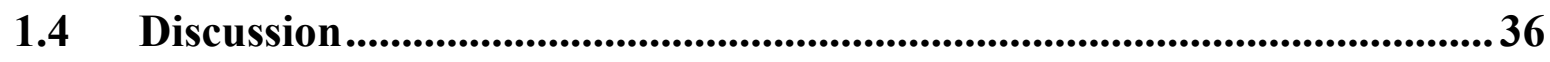

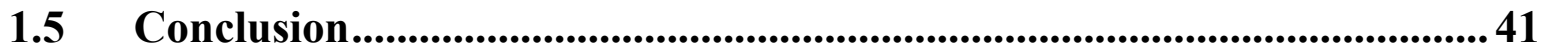

2 Processing pronominal pointing signs in German Sign Language:

Neurophysiological evidence for the first mention effect ................................43

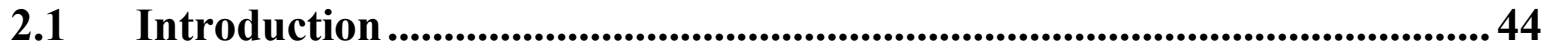

2.1.1 First mention effects in spoken languages ....................................... 45

2.1.2 Pronoun resolution strategies in sign languages ...................................... 46 
2.1.3 First mention effects and subject preference in sign languages .............. 49

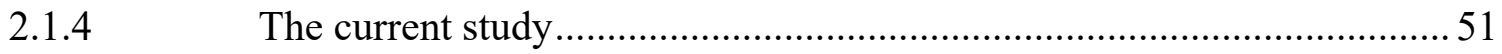

2.2 Methods ..................................................................................................55

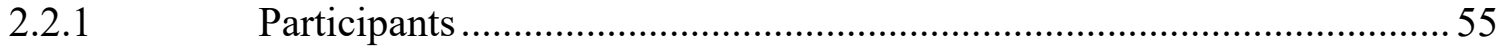

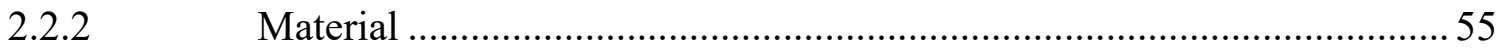

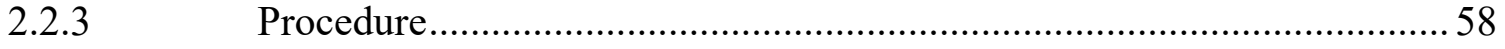

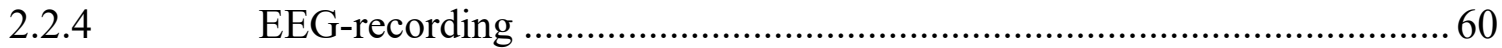

2.3 Results ..................................................................................................61

2.4 Discussion.................................................................................................62 62

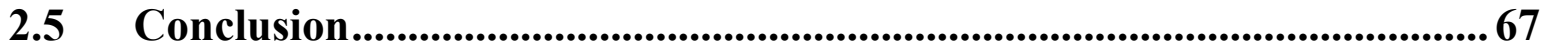

3 The influence of overt localization on the processing of referential expressions in German Sign Language ................................................................69

3.1 Introduction ............................................................................................. 70

3.1.1 Prominence and its effects on processing in spoken languages .............. 70

3.1.2 Prominence and its effects on processing in sign languages................... 73

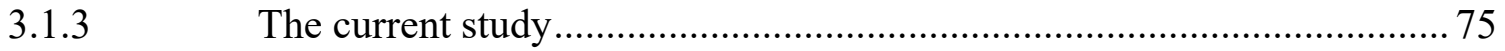

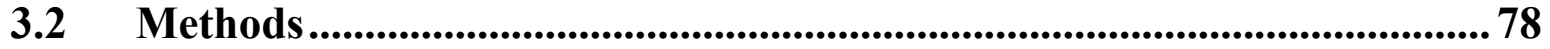

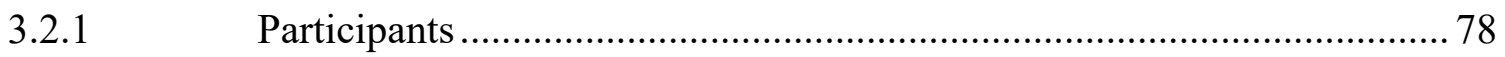

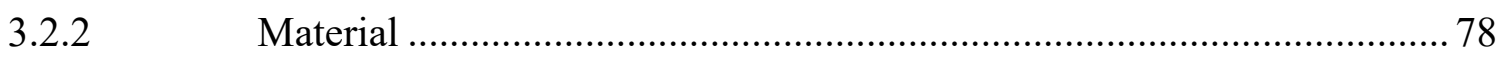

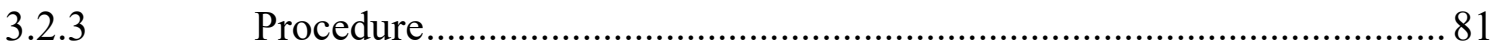

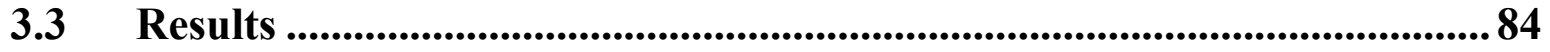

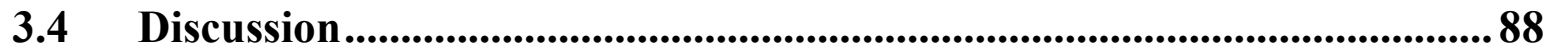

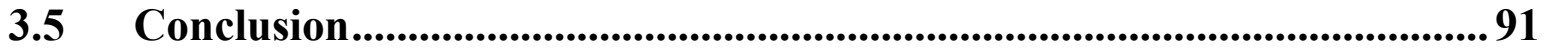

4 Phonological priming in German Sign Language: An eye tracking study using the Visual World Paradigm ...........................................................93

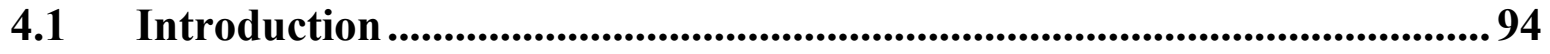

4.1.1 Effects of phonological parameters ..................................................... 95

Eye tracking studies in sign languages................................................. 99

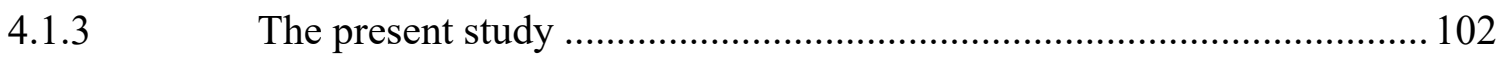

4.2 Methods .......................................................................................................... 104

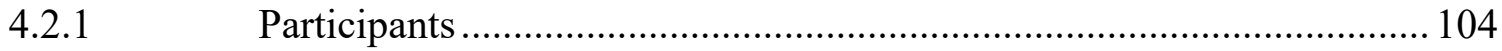

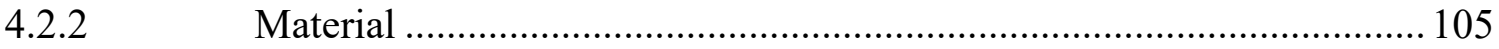




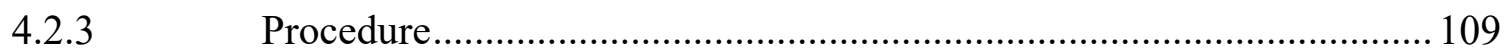

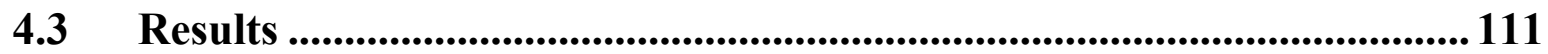

4.4 Discussion .............................................................................................. 114

4.5 Conclusion.................................................................................................... 119

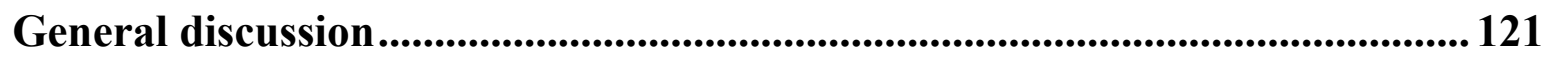

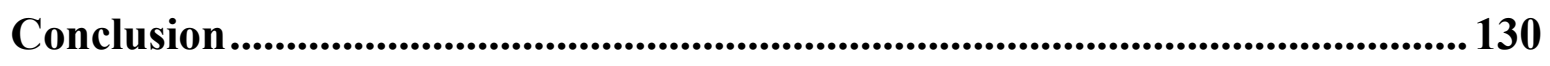

References ....................................................................................................................I

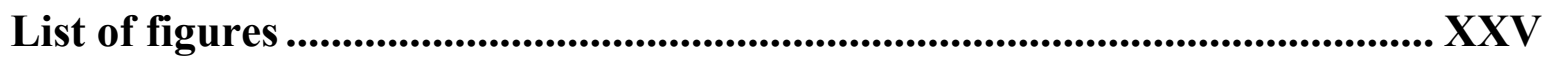

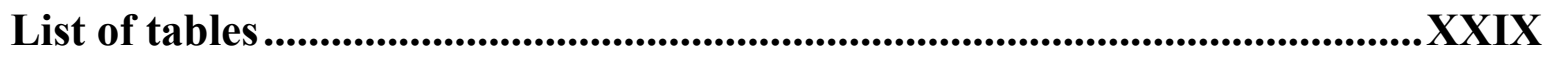

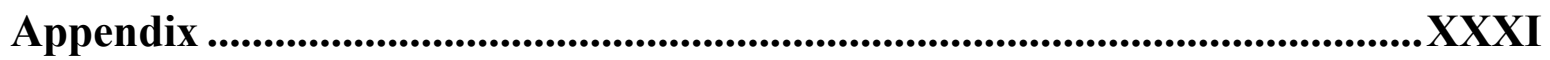

Selbstständigkeitserklärung ...............................................................................LXVII 



\section{General introduction}

Sign language research has its origin in the 1960's in the work of William C. Stokoe on American Sign Language (Stokoe, 1960). Ever since, the striking question is whether the modality of a language, i.e., visual-manual compared to auditory-articulatory, affects its processing, and if so, in what respect. Therefore, psycholinguistic sign language research aims to determine where the similarities and the differences between sign and spoken language processing can be seen. So far, our understanding of language processing in general and accounts modelling these processes are based on spoken language data. Investigating languages using a different modality allows us to examine the universality of mechanisms underlying language processing. Moreover, models accounting for spoken language processing phenomena can be tested regarding their validity by examining their application to sign language data. But what is so special about the sign language modality that makes it increasingly more attractive for (psycho-)linguistic research?

The primary focus of this thesis is how the usage of the signing space can realize various phonological and morphosyntactic as well as semantic and pragmatic functions. The signing space denotes the space in front of a signer, including the head and the upper part of the body (see Figure 1 in Chapter 1), and consists of three different planes: (i) frontal, (ii) midsagittal, and (iii) horizontal. The frontal or ventral plane indicates hierarchical relations (Schlenker et al., 2013) and information structure (Wilbur, 2012; Wilbur \& Patschke, 1998), expresses specificity (Barberà, 2012) and is used for role shift (Herrmann \& Steinbach, 2007; Lillo-Martin, 2012). The midsagittal plane expresses distance of entities of situations and temporal reference (Emmorey, 2002; Sandler, 1989). Finally, the horizontal, or transverse, plane is used to mark agreement (Padden, 1990), plurality (Pfau \& Steinbach, 2006), 
reciprocal relations (Pfau \& Steinbach, 2003) and contrast (van der Kooij et al., 2006), to express temporal reference and topographic relations, to realize various morphosyntactic, semantic and pragmatic functions (Emmorey, 2002), express role shift and to link discourse referents (Lillo-Martin \& Klima, 1990).

The distinction between the topographic and the abstract (also referred to as syntactic or referential) function of the space (Klima \& Bellugi, 1979; Liddell, 1990, 1995; Poizner et al., 1987) is a subject of ongoing debate. The topographic function indicates spatial relations among known entities, therefore the locations in the signing space represent actual physical locations. In contrast, the abstract function reflects an abstract use of the signing space where entities are assigned to arbitrary locations that derive their meaning from the current discourse (Emmorey et al., 1995; Poizner et al., 1987). This view, that such a distinction applies, is supported by various studies. Data obtained from patients with brain lesions show a double dissociation regarding the two functions. While right-lesioned patients have problems describing spatial relations of objects, they show correct use of the abstract space, and where left-lesioned patients succeed in correctly using spatial descriptions, they have difficulty using the abstract space (Poizner et al., 1987). Additional psycholinguistic studies have shown that each respective use of the space is processed differently, and this is reflected by the differences in accuracy and the reaction times of the answers provided (Emmorey et al., 1995). Moreover, referential expressions activate their antecedents, but not the spatial location (Emmorey et al., 1995; Emmorey \& Falgier, 2004). However, locations on the horizontal plane can take both functions at the same time suggesting that these themselves are not mutually exclusive.

For physically present discourse referents, signers can refer to them by pointing directly to their actual physical locations. In contrast, referential locations (henceforth R-loci; 
Liddell, 1990; Lillo-Martin \& Klima, 1990) need to be established for referents that are not physically present to refer to them in subsequent discourse. Even if there is an infinite number of possible R-loci, only two to three different locations are used at a time due to cognitive limitations (Lillo-Martin \& Meier, 2011). Theoretical frameworks analyze R-loci and their functions from different persepctives (for an overview, see Sandler \& Lillo-Martin (2006)). Syntactic framworks define locations used as syntactic argument marking to distinguish between subject and object. Thus, referential indices carried by referential expressions and their antecedents are copied during syntactic agreement and licensed under syntactic conditions and morphologically realized as a part of verbal agreement (Aronoff et al., 2005). Feature-based accounts suggest R-loci to represent markings of person phi-features or pronominal features (Klima \& Bellugi, 1979; Lillo-Martin \& Klima, 1990; Neidle et al., 2000). A semantic approach is provided by Steinbach \& Onea (2016). The authors suggest an analysis of the grammatical anaphoric space based on a system of recursive features integrated in the framework of the discourse representation theory (Kamp \& Reyle, 1993) that allows for an indefinite number of R-loci. These features account for the geometrical properties of the signing space used for the implementation of referential relations. Steinbach \& Onea (2016) assume each discourse referent is assigned to a unique R-loci, a linguistic entity interpreted in discourse semantics. While semantic and pragmatic principles govern the assignment of referents to an R-loci, the principle of opposition governs the distribution of Rloci in the horizontal plane following the right-left default pattern described below.

Discourse referents are assigned to an arbitrary location using manual and nonmanual strategies either separately or in combinations. Manual strategies include classifier predicates (Liddell, 1990), verbal agreement (Winston, 1996), phonological adaptation of non bodyanchored signs, i.e., producing a sign directly at the locus in the space (Lillo-Martin, 1986), or pointing with the index finger (INDEX sign) (Liddell, 1990; Lillo-Martin, 1986; Winston, 
1996). Nonmanual strategies use body leans (Winston, 1996) or eye gaze movement directed towards a locus (Lillo-Martin, 1986; Winston, 1996). Moreover, discourse referents can be assigned to an R-locus using an implicit strategy that does not require any spatial device. This implicit assignment follows a particular pattern using contrastive locations as suggested by Steinbach \& Onea (2016). They propose the so-called right-left default pattern that associates the first mentioned referent with the right (ipsilateral) and the second referent with the left (contralateral) side of the signing space for right-handed signers. Other researchers have reported a similar pattern for various sign languages before. For Catalan Sign Language (LSC), Barberà (2012) observes a tendency to link the first referent to the ipsilateral side. However, the ipsilateral side refers to the side that is the closest to the dominant hand of a signer. Thus, right-handed signers would use the side to their right while left-handed signers would choose the side to their left for localizing the first discourse referent. According to Geraci (2014), signers of Italian Sign Language (LIS) assign the subject of a sentence, i.e., the first mentioned referent since it is an SOV language, on the side of their dominant hand, which is in parallel to LSC signers. Additionally, Herrmann \& Steinbach (2007) mention that right-handed signers of German Sign Language (DGS) usually place the first referent on their right side as well. For further sign languages, the use of contrastive locations using ipsi- and contralateral areas was described as well without specifiying an order of referent assignment (American Sign Language (ASL) (Sandler \& Lillo-Martin, 2006; Schlenker, 2013), British Sign Language (BSL) (Sutton-Spence \& Woll, 1999), Israeli Sign Language (ISL) (Meir \& Sandler, 2008) and Danish Sign Language (DSL) (Engberg-Pedersen, 1993)).

Referential expressions in sign languages take various forms and their use varies depending on the referential context they occurr in (McKee et al., 2011; Wulf et al., 2002). During production, the use of referential expression overall follows the referential hierarchy proposed for spoken languages (Ariel, 1988; Gundel et al., 1993; among others), nevertheless, 
showing modality-specific modifications especially regarding the use of zero forms or classifiers (Frederiksen \& Mayberry, 2016). Overt lexical pronouns are pointings using the index finger (INDEX $\operatorname{sign}^{1}$ ) directed either to the referent or to previously established R-loci to refer to a third person referent ${ }^{2}$ (Lillo-Martin \& Klima, 1990; for an overview, see Cormier, 2012). They are assumed to be unambiguous in their singular form, but in certain contexts it is possible that a direct relation of pronoun and referent is not given resulting in ambiguity (McBurney, 2002). Number on pronouns is expressed by additional movements, e.g., an arcshaped movement, or by replacing the index finger handshape with a numeral handshape. Additionally, there are reports about distinction of inclusive and exclusive in the first person in ASL and BSL (Cormier, 2005, 2007; McBurney, 2002). Gender marking on pronouns is rarely observed and was only suggested for Asian Sign Languages (for an overview, see McBurney, 2002). Analyzing corpus data, overt pronominal marking is most likely to occur when reference switches, i.e., when the pronoun is not co-referent with the subject of the previous sentence (McKee et al., 2011; Wulf et al., 2002). For DGS, overt pronouns are more likely to occur during referent maintenance (P. M. Perniss \& Özyürek, 2015). In contrast, bare nouns, i.e., not combined with another sign to form a complex noun phrase, are mostly used to introduce referents to set the context (Frederiksen \& Mayberry, 2016; Sandler \& LilloMartin, 2006).

\footnotetext{
${ }^{1}$ Pointing signs function as determiners as well when co-occurring with a noun (MacLaughlin, 1997; Sandler \& Lillo-Martin, 2006).

${ }^{2}$ First person is expressed by directing a pronoun toward the signer's chest, while direction towards the addressee's chest indicates second person (for an overview, see (McBurney, 2002)). It is an ongoing debate whether pronominal systems show a three-way, i.e., first, second and third person, or a two-way, i.e., first and non-first person, distinction (Engberg-Pedersen, 1993; Friedman, 1975; Meier, 1990).
} 
Null arguments licensed by the spatial modification of verbal agreement ${ }^{3}$ (LilloMartin, 1986; Neidle et al., 2000) are the most frequently used. Across different sign languages, null forms occurr mostly in the context of maintaining and re-introducing referents (Frederiksen \& Mayberry, 2016; McKee et al., 2011; P. M. Perniss \& Özyürek, 2015; Wulf et al., 2002). This kind of implicit reference could be due to agreement features of the verb that identify subject and object by marking the starting and endpoint, either single or both, of a movement, which depends on the specific agreement type, i.e., double or single agreement. Thus, when a referent has been established at a specific location, no additional explicit reference is necessary in the context of an agreement verb (Friedman, 1975). The same function can be fulfilled by classifiers, i.e., handshapes representing a referent itself or how this referent is handled, or by classifier predicates, i.e., combining a classifier with a movement or location in space representing specific information about the referent (Boyes Braem, 1995; P. M. Perniss, 2007).

A vast literature on spoken languages discusses the influence of various factors on the processing of referential expressions (for an overview, see Almor \& Nair, 2007). Factors such as word order, verb semantics or gender marking modulate the functionality of referential expressions and thus might cause increasing processing costs due to additional involvement of the working memory (Arnold et al., 2000; McKoon et al., 1996; van Berkum et al., 2007). Moreover, properties of the antecedent such as salience and information structural status affect the resolution of reference relations (Almor \& Eimas, 2008; Ariel, 1990; Givón, 1976). The investigation of these factors has been neglected in sign language research and it is therefore unclear what factors affect the processing of referential expressions.

\footnotetext{
${ }^{3}$ Therefore, analyses of sign languages as pro-drop languages in general is suggested based on cross-
} linguistically similar observed structures (Lillo-Martin, 1986; Lillo-Martin \& Meier, 2011; Padden, 1990). 
Studies examined sign language processing using various tasks such as gating, signpicture interference or lexical decisions (Baus et al., 2008, 2014; Carreiras et al., 2008; Emmorey \& Corina, 1990; Grosjean, 1980; Orfanidou et al., 2009). Moreover, investigations include different techniques and different groups of participants (MacSweeney et al., 2008; for an overview, see Emmorey, 2003). Often, neuroimaging studies explore whether the same neural networks underlie spoken and sign language processing by using fMRI (functional magnetic resonance imaging), PET (positron emission tomography) and MEG (magnetoencephalography) (Capek et al., 2008; Emmorey et al., 2013, 2014; Leonard et al., 2012; Levänen et al., 2001; MacSweeney et al., 2002). The participants tested include monolingual deaf native signers and bimodal bilinguals, i.e., hearing native signers, all of whom might differ as well with regard to age of acquisition (MacSweeney, Waters, et al., 2008; Mayberry \& Witcher, 2005; Poizner, 1983).

Starting in the late 1980's, Kutas et al. (1987) conducted the first event-related potentials (ERP) study investigating effects of semantic anomalies in sign language sentence processing in ASL. Their study revealed an N400 effect in response to written, spoken and signed stimuli suggesting that underlying processes are modality independent. Besides replicating this effect, Neville et al. (1997) provided evidence for the influence of age of acquisition on this effect showing earlier and more long lasting effects for native signers. In both studies, sentences were presented sign by sign, thus questioning the validity of the results regarding sentence processing. However, Capek et al. (2009) and Grosvald et al. (2012) confirmed the N400 as response to semantic anomalies during sentence processing by using a more natural way of sentence presentation. In contrast, (morpho-) syntactic violations elicited a biphasic pattern of (E)LAN and P600 (Capek et al., 2009). For DGS, similar effects were observed by Hänel-Faulhaber et al. (2014) and Hosemann et al. (2013). Most importantly, the effects were observed prior to sign onset in the study by Hosemann et al. (2013) suggesting 
that the transition phase between two signs carries sufficient information to trigger predictive processing. The work by Krebs $(2013,2017)$ on Austrian Sign Language (ÖGS) supports this finding and provides evidence that word order variations evoke different effects that might be triggered by nonmanual markings occurring in the transition phase. Table 0.1 below presents an overview of selected ERP studies on various sign languages.

Table 0.1.1 Overview of selected ERP studies on sign languages

Main findings and the investigated languages for each study are presented. More detailed descriptions of the studies can be found throughout Chapter $1-4$.

\begin{tabular}{|c|c|c|}
\hline authors & language & main findings \\
\hline $\begin{array}{l}\text { Kutas et al. } \\
\text { (1987) }\end{array}$ & $\begin{array}{l}\text { ASL } \\
\text { (\& English) }\end{array}$ & $\begin{array}{l}\text { Semantic anomalies in a sentence evoke the same N400 } \\
\text { effect in written, spoken and sign language. }\end{array}$ \\
\hline $\begin{array}{l}\text { Neville et al. } \\
\text { (1997) }\end{array}$ & $\begin{array}{l}\text { ASL } \\
\text { (\& English) }\end{array}$ & $\begin{array}{l}\text { Semantic violations evoke an N400 in deaf native } \\
\text { signers, hearing late signers, but not in hearing non- } \\
\text { signers. Earlier onset of the effect and longer lasting in } \\
\text { deaf native signers. }\end{array}$ \\
\hline $\begin{array}{l}\text { Capek et al. } \\
(2009)\end{array}$ & ASL & $\begin{array}{l}\text { Semantic anomalies elicited an N400 while syntactic } \\
\text { violations elicited LAN + P600 }\end{array}$ \\
\hline $\begin{array}{l}\text { Grosvald et al. } \\
(2012)\end{array}$ & ASL & $\begin{array}{l}\text { Incongruent signs and pseudo-signs evoke an N400 } \\
\text { while gestures evoke a large positivity ( } 600-800 \mathrm{~ms})\end{array}$ \\
\hline $\begin{array}{l}\text { Gutiérrez et al. } \\
\text { (2012) }\end{array}$ & LSE & $\begin{array}{l}\text { N400 effect for phonologically related signs sharing the } \\
\text { location parameter, but later negativity }(600-800 \mathrm{~ms}) \\
\text { for non-signs sharing the same handshape parameter }\end{array}$ \\
\hline $\begin{array}{l}\text { Gutiérrez et al. } \\
\text { (2012) }\end{array}$ & ASL & $\begin{array}{l}\text { Early negativity }(150-250 \mathrm{~ms}) \text { for semantically related } \\
\text { signs; N400 ( } 350-450 \mathrm{~ms}) \text { for either semantically or } \\
\text { phonologically related signs; N400 ( } 450-600 \mathrm{~ms}) \text { for all } \\
\text { conditions compared to baseline. }\end{array}$ \\
\hline
\end{tabular}




\begin{tabular}{|l|l|l|}
\hline $\begin{array}{l}\text { Hosemann et al. } \\
\text { (2013) }\end{array}$ & DGS & $\begin{array}{l}\text { Semantically unexpected signs evoke a biphasic pattern } \\
\text { of N400 and late positivity triggered by properties of } \\
\text { the transition phase, i.e., prior to the onset of the } \\
\text { unexpected sign. }\end{array}$ \\
\hline $\begin{array}{l}\text { Hänel-Faulhaber } \\
\text { et al. (2014) }\end{array}$ & DGS & $\begin{array}{l}\text { Morphosyntactic violations evoke an ELAN + broad } \\
\text { P600 and semantic violations evoke a frontal N400. }\end{array}$ \\
\hline DGS & $\begin{array}{l}\text { Phonologically related signs, even cross-modal and } \\
\text { cross-language, elicit a reduced N400 compared to } \\
\text { unrelated signs. } \\
\text { Effects evoked by agreement violations differ between } \\
\text { plain verbs (broad positive deflection from 420-730 ms) } \\
\text { and agreement verbs (posterior positivity from 220-570 } \\
\text { and left anterior effect from 300-600 ms). }\end{array}$ \\
\hline Krebs (2017) & ÖGS & $\begin{array}{l}\text { OSV word order elicits more pronounced negativity and } \\
\text { positivity effects compared to basic SOV word order in } \\
\text { declarative sentences and in sign language specific topic } \\
\text { and classifier constructions. These effects are triggered } \\
\text { during the transition phase, possibly due to nonmanual } \\
\text { markings. }\end{array}$ \\
\hline
\end{tabular}

Eye movements provide insight into mechanisms underlying language comprehension that can be observed under natural conditions using the eye tracking technique (Cooper, 1974; Tanenhaus et al., 1995; for an overview, see Huettig et al., 2011). Up to now, there are only a few eye tracking studies investigating sign languages in general (for an overview of selected studies, see Table 0.2). This research started looking at sign language production by examining the relation of eye gaze and agreement across different sign languages and the influence of age of acquisition on this relation (Hosemann, 2011; Thompson et al., 2006, 2009). Other studies used this technique to determine where signers direct their eye gaze while perceiving sign language showing that the more proficient a signer is, the more the face gets fixated, especially the eyes (Emmorey et al., 2009; Muir \& Richardson, 2005). In spoken 
language research, eye tracking technique is often combined with the Visual World Paradigm (Huettig et al., 2011; Tanenhaus et al., 1995). In contrast, only three studies used this paradigm in sign language research addressing different topics. While Lieberman et al. (2015) investigate effects during the processing of isolated signs, Thompson et al. (2013) used single sentences always consisting of the same carrier phrase combined with a changing target sign. The study by Lieberman et al. (2015) provides additional evidence for predictive processing in sign language. Regarding their experimental setting, these three studies modified the classical Visual World Paradigm to fulfill the needs of a visually conveyed language. Therefore, all studies presented a video in the middle of a screen surrounded by four pictures varying their relation to the video. This setting is modified further for the purpose of the studies conducted for this thesis.

Table 0.1.2 Overview of selected eye tracking studies on sign languages

The main findings and the investigated languages of each study are presented. More detailed descriptions of the studies can be seen throughout Chapter $1-4$.

\begin{tabular}{|l|l|l|}
\hline \multicolumn{1}{|c|}{ authors } & language & \multicolumn{1}{c|}{ main findings } \\
\hline $\begin{array}{l}\text { Muir \& } \\
\text { Richardson } \\
(2005)\end{array}$ & BSL & $\begin{array}{l}\text { When watching sign language video clips, the face of a signer } \\
\text { is mostly fixated. }\end{array}$ \\
\hline $\begin{array}{l}\text { Thompson et } \\
\text { al. (2006) }\end{array}$ & ASL & $\begin{array}{l}\text { Agreement verbs are accompanied by eye gaze towards the } \\
\text { syntactic object, while eye gaze combined with spatial verbs } \\
\text { was directed towards the locative argument rather than the } \\
\text { syntactic subject or object. Moreover, the height of the eye } \\
\text { gaze differed between these verb types suggesting differences } \\
\text { in the argument type. }\end{array}$ \\
\hline
\end{tabular}




\begin{tabular}{|c|c|c|}
\hline $\begin{array}{l}\text { Emmorey et al. } \\
(2009)\end{array}$ & ASL & $\begin{array}{l}\text { Overall, beginning sign language learners and native signers } \\
\text { fixate on the other signer's face. However, native signers } \\
\text { primarily fixated on the signer's eyes while beginning sign } \\
\text { language learners fixate the mouth and shift their gaze more } \\
\text { often to the signer's hands as well. }\end{array}$ \\
\hline $\begin{array}{l}\text { Thompson et } \\
\text { al. (2009) }\end{array}$ & ASL & $\begin{array}{l}\text { While native and proficient L2 signers show the same gaze } \\
\text { pattern with agreement verbs marking the object and with } \\
\text { spatial verbs directing the eye gaze towards the locative } \\
\text { argument, they also show differences for plain verbs with } \\
\text { more object markings by the proficient L2 signers. Beginning } \\
\text { L2 signers do not show a consistent pattern except that they } \\
\text { tend to use the eye gaze more than non-signers. }\end{array}$ \\
\hline $\begin{array}{l}\text { Hosemann } \\
(2011)\end{array}$ & DGS & $\begin{array}{l}\text { Agreement and spatial verbs are combined with eye gaze } \\
\text { marking the syntactic object or locative argument. However, } \\
\text { eye gaze patterns in DGS are not as systematic as in ASL. }\end{array}$ \\
\hline \multicolumn{3}{|r|}{ Studies using the Visual World Paradigm } \\
\hline $\begin{array}{l}\text { Thompson et } \\
\text { al. (2013) }\end{array}$ & BSL & $\begin{array}{l}\text { Semantic and phonological effects during online processing } \\
\text { showing more looks to a related distractor, but the } \\
\text { phonological effect differs based on the phonological } \\
\text { properties of the target and the distractor. }\end{array}$ \\
\hline $\begin{array}{l}\text { Lieberman et } \\
\text { al. (2015) }\end{array}$ & ASL & $\begin{array}{l}\text { Native signers show activation of semantic and phonological } \\
\text { features during sign processing. Late signers show this } \\
\text { activation somewhat later during post-lexical comprehension. }\end{array}$ \\
\hline $\begin{array}{l}\text { Lieberman et } \\
\text { al. (2017) }\end{array}$ & ASL & $\begin{array}{l}\text { Deaf adults and children show anticipatory looks to a target in } \\
\text { the presence of semantically constraining information } \\
\text { suggesting that both groups make predictions about the } \\
\text { upcoming language input. }\end{array}$ \\
\hline
\end{tabular}

This thesis aims to investigate modality-independent and modality-specific factors influencing referential processing relying on theoretical proposal provided by Steinbach \& Onea (2016) and using different experimental methods. Each of the subsequent four chapters is, or will be, submitted to scientific journals as individual co-authored papers for publication. 
The ERP study presented in Chapter 1 (Wienholz, Nuhbalaoglu, Mani, et al., 2018) examines the default pattern applying in the absence of overt localization of discourse referents used to assign these referents to R-loci on the horizontal plane. Then, using the same method, Chapter 2 (Wienholz et al., submitted) determines whether a first mention effect is guiding the comprehension of pronominal reference using the same ambiguous contexts as in the previous chapter. The following chapters 3 and 4 use the eye tracking technique combined with the Visual World Paradigm. Chapter 3 (Wienholz, Nuhbalaoglu, Steinbach, et al., 2018) addresses the question of the influence of overt localization on the processing of full/bare nouns. Finally, Chapter 4 (Wienholz et al., 2021) examines the phonological priming effect at the sentence level and the influence of single phonological parameters on this effect. 


\title{
1 Pointing to the Right Side? An ERP Study on Anaphora Resolution in \\ German Sign Language
}

\begin{abstract}
Sign languages use the horizontal plane to refer to discourse referents introduced at referential locations. However, the question remains whether the assignment of discourse referents follows a particular default pattern as recently proposed such that two new discourse referents are respectively assigned to the right (ipsilateral) and left (contralateral) side of (right handed) signers. The present event-related potential study on German Sign Language investigates the hypothesis that signers assign distinct and contrastive referential locations to discourse referents even in the absence of overt localization. By using a semantic mismatch-design, we constructed sentence sets where the second sentence was either consistent or inconsistent with the used pronoun. Semantic mismatch conditions evoked an N400, whereas a contralateral INDEX sign engendered a Phonological Mismatch Negativity. The current study provides supporting evidence that signers are sensitive to the mismatch and make use of a default pattern to assign distinct and contrastive referential locations to discourse referents.
\end{abstract}

Keywords: German Sign Language, event-related potentials, semantic mismatch, anaphora resolution, $\mathrm{N} 400$ 


\subsection{Introduction}

Natural languages come in two modalities, the oral-auditory modality of spoken languages and the visual-gestural modality of sign languages. Although the two modalities clearly differ in production and perception of communicative signals, their underlying linguistic structures seem to be very similar (Meier, 2002, 2012; Sandler \& Lillo-Martin, 2006). One apparent difference is that sign languages exploit the signing space, which is the space in front of the signer's upper part of the body and the head (Figure 1.1A), to realize various phonological, morphosyntactic, semantic, and pragmatic functions.

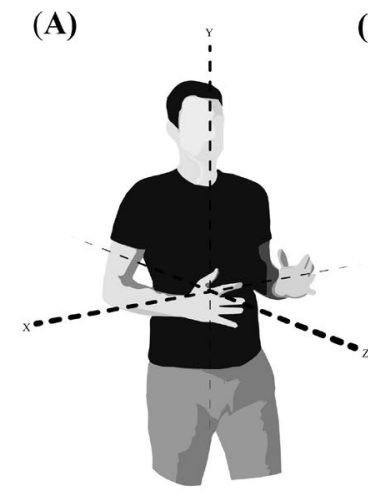

C) SLG GTGN LAB

(B)

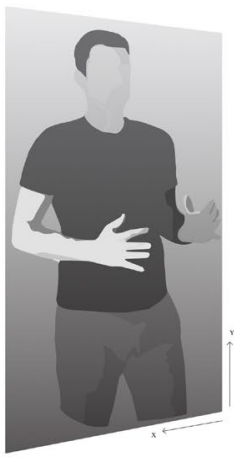

frontal

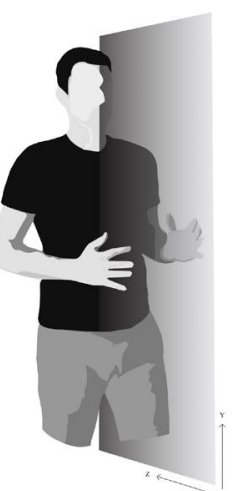

midsaggital

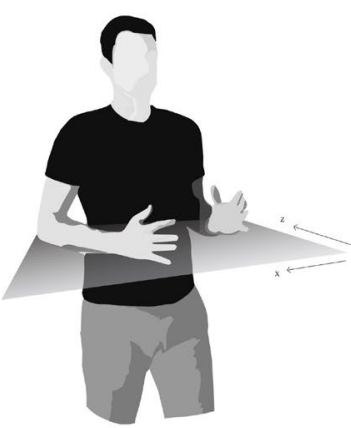

horizontal

Figure 1.1 Signing space (A) and its planes (B)

This space can be subdivided into three different planes (Brentari, 1998): frontal, midsaggital and horizontal (Figure 1.1B). Crucial for the current study is the horizontal plane, which is used to indicate agreement (Padden, 1990), contrast (van der Kooij et al., 2006), plurals (Pfau \& Steinbach, 2006), reciprocals (Pfau \& Steinbach, 2003), role shift (Herrmann \& Steinbach, 2007; Lillo-Martin, 2012), temporal reference and topographic relations 
(Emmorey, 2002), as well as for linking discourse referents (DRs) (Lillo-Martin \& Klima, 1990).

\subsubsection{Discourse referents and referential locations}

In spoken languages, a discourse repeatedly refers to certain referents by using anaphoric expressions such as pronouns, e.g., English he, she, or it (Ariel, 1990, 2001). Similarly, sign language signers use, for instance, spatial pronouns, e.g., pointing with the index finger, to refer back to DRs (Sandler \& Lillo-Martin, 2006).

Using the signing space to localize DRs varies based on whether referents are physically present or not. For physically present referents, signers can easily identify these by pointing to them and their actual physical location. However, if referents are not physically present, a DR has to be associated with an arbitrary locus on the horizontal plane. This area is called a referential locus (henceforth, R-locus; Liddell, 1990; Lillo-Martin \& Klima, 1990). Once a referent is linked to a locus, pronominal signs can be directed toward that locus to refer back to that referent in the subsequent discourse - at least until signers change or overwrite the R-locus associated with this referent (Lillo-Martin \& Klima, 1990; Poizner et al., 1987; Sandler \& Lillo-Martin, 2006). Various constraints can influence the choice of the locus including semantic or logical relations between referents (e.g., family members may be expressed at the same locus (Klima \& Bellugi, 1979)) or pragmatic considerations (i.e., overtly assigning a locus to a referent emphasizes its importance (Engberg-Pedersen, 1993)).

Signers can use a variety of overt manual and non-manual strategies, either separately or in combination, to assign referents to an R-locus. One strategy among the manual options that use the hands for referent assignment is pointing with the index finger (INDEX sign) to a 
R-locus (Liddell, 1990; Lillo-Martin, 1986; Winston, 1996). In contrast, non-manual strategies include body leans or directing eye-gaze towards a R-locus (Lillo-Martin, 1986; Winston, 1996). Moreover, DRs can be implicitly assigned to "default" R-loci, as described in the following section.

\subsubsection{Localizing discourse referents in signing space}

Steinbach and Onea (Steinbach \& Onea, 2016) discuss a default that appears to govern the assignment of DRs to particular R-loci. First, they note that each DR is linked to a unique and distinct R-locus, which is then used to represent this DR in the discourse representation structure (DRS). Second, they propose the principle of opposition saying that DRs are introduced into maximally contrastive regions in the horizontal space.

Importantly for the purposes of this study, they note that a default pattern, henceforth called right-left default pattern, might constrain the linking of DRs to R-loci. The principle of opposition ensures maximal spatial contrast of the first two DRs. In addition to manual and non-manual strategies signers can use an implicit default strategy that does not include any spatial device. In this case, referents are not explicitly assigned to a particular R-locus. Rather, both the signer and the addressee implicitly associate referents with and refer to this R-locus in subsequent discourse when referring to corresponding DRs. According to the default - at least for right-handed signers - the first referent is typically linked to the right (ipsilateral), and the second to the left (contralateral) side of the signing space.

Support for such a default is reported in several languages, including Catalan, Italian, German and American Sign Language. Thus, in Catalan Sign Language, Barberà (Barberà, 2012) observes a tendency for 'signers (to) use their corresponding ipsilateral part to establish 
the first location' (p.103), potentially because this location is closer to the dominant hand (of right-handed signers). In Italian Sign Language, Geraci (Geraci, 2013) claims that subjects (first referents since Italian Sign Language is an SOV language) are placed on the side of the dominant hand, such that for right-handed signers, the first referent would be localized on the right side. A similar pattern is reported for a number of languages (German Sign Language (DGS) (Herrmann \& Steinbach, 2007), American Sign Language (ASL) (Sandler \& LilloMartin, 2006; Schlenker, 2013) and Israeli Sign Language (Meir \& Sandler, 2008)). Studies of other sign languages highlight the use of opposing ipsi- and contralateral sides while remaining silent about positioning first and second DRs to particular sides (British Sign Language (Sutton-Spence \& Woll, 1999) and Danish Sign Language (Engberg-Pedersen, 1993)).

\subsubsection{Antecedent reactivation in spoken and sign languages}

Several studies have investigated antecedent (re-)activation during processing of referential expressions in spoken languages. For instance, in their review Nicol \& Swinney (1989) show that antecedent reactivation is influenced either by structural (e.g., syntactic relations between antecedent and referential expression) or pragmatic (e.g., salience) information during processing of either explicit (e.g., pronouns) or implicit (e.g., null pronouns) anaphoric expressions. They emphasize that especially structural information cause immediate reactivation of matching antecedents. In case of ambiguous antecedents, all possible referents are simultaneously activated and pragmatic information helps to identify the correct antecedent, although this applies only at a later stage of comprehension (cf. Nicol \& Swinney (1989) for further references). 
For sign languages, Emmorey et al. (1991) suggest that antecedent activation appears to require more time relative to spoken languages. They presented signed sentences of ASL containing two possible referents and measured the response times to probe signs presented immediately or with a delay after the critical sign (pronoun or nominal referent) in a second sentence. Response times to probe signs only differed at the later probe sign presentation with faster responses to the pronoun. These results suggest that corresponding referents of the pronoun have only been activated at the delayed probe sign presentation, although the authors suggest that referent activation may occur earlier than measured in the current task (Emmorey \& Corina, 1990). Indeed, subsequent experiments suggested that non-antecedent suppression occurs earlier in sign languages (Emmorey, 1997). The authors suggest that this effect may be due to the unambiguous nature of pronouns in ASL, given that pronouns refer to unique spatial locations associated with referents in sign language (Emmorey et al., 1991).

Therefore, Emmorey \& Falgier (2004) investigated the case of locus doubling (i.e., associating a single referent with two distinct spatial loci) to examine whether pronouns in ASL activate both their antecedents and the corresponding spatial locations. They presented sentences that associated a referent with two distinct spatial locations continuing with a second sentence containing a pronoun or no anaphoric element and measured response times to probe signs (referent and both locations). Results showed faster response times to referent probes following a pronoun compared to a control, but no difference for both location probes. The findings suggest that ASL pronouns only activate their antecedent but not the spatial location the antecedent is associated with.

Taken together, these studies suggest that pronouns (either overt or phonologically empty) reactivate their antecedent referents (Emmorey et al., 1991; Emmorey \& Lillo-Martin, 1995), but not their spatial location (Emmorey \& Falgier, 2004), thus providing evidence for 
similarity of antecedent activation in sign and spoken languages. Based on these findings, Emmorey (Emmorey, 2007) suggests that processing mechanisms involved in resolving and interpreting co-referential relations are the same cross-linguistically and therefore modalityindependent. Nevertheless, these studies highlight differences in the timing of antecedent activation and non-antecedent suppression across sign and spoken languages requiring separate investigation.

\subsubsection{The current study}

The current study investigates to which extent a default pattern of linking DRs to Rloci in the horizontal plane exists and whether this default pattern influences processing of DRs in DGS. We examine whether signers assign distinct and contrastive R-loci to different DRs and, critically, whether right-handed signers implicitly and automatically assign the first referent to the right (ipsilateral) and the second referent to the left (contralateral) side.

Participants are presented with short discourses, which included introducing two new DRs in the first sentence (e.g., MAN and WOMAN ${ }^{4}$ in the subsequent examples- for video stills see Appendix A). Neither referent is explicitly assigned to an R-locus - thus, the addressee has to rely on default patterns governing the assignment of DRs to R-loci. Afterwards, a second sentence begins with the pointing sign INDEX directed to either the right/ipsilateral (INDEX 3 a) or left/contralateral (INDEX 3 b) side. The INDEX functions as a pronoun in this case

\footnotetext{
${ }^{4}$ By convention, signs are glossed in small caps. In the discussed examples, index is always used as a
} pronoun to refer back to one of the two DRs. Subscript number ' 3 ' refers to the R-locus and subscripts 'a' (right/ipsilateral) and ' $b$ ' (left/contralateral) refer to the corresponding region in the horizontal plane. Note that DGS is an SOV language. 
and, based on whether it is directed to the ipsilateral or contralateral side, anaphorically picks up a DR that has been previously assigned to the respective R-locus. Then a semantically neutral sign (HAVE) and a semantically biased sign (BEARD), that is only consistent with one of the referents in the first sentence (MAN), and inconsistent with the other one (WOMAN), follow.

Condition 1:

MAN WOMAN FLIRT. INDEX3a HAVE BEARD.

'A man flirts with a woman. He has a beard.'

Condition 2:

MAN WOMAN FLIRT. INDEX3b HAVE BEARD.

'A man flirts with a woman. She has a beard.'

Condition 3:

WOMAN MAN FLIRT. INDEX3a HAVE BEARD.

'A woman flirts with a man. She has a beard.'

Condition 4:

WOMAN MAN FLIRT. INDEX3b HAVE BEARD.

'A woman flirts with a man. He has a beard.'

Given reports of the right-left default pattern, we hypothesize that participants implicitly assign the first referent to the ipsilateral side, i.e., MAN would be assigned to the ipsilateral locus (INDEX 3 a) in Conditions 1 and 2. By contrast, WOMAN would be assigned to the ipsilateral locus (INDEX 3 a) in Conditions 3 and 4. Thus the disambiguating noun (BEARD) 
would be consistent with the antecedent in Conditions 1 and 4 . Based on the default pattern, the ipsilateral locus (INDEX 3 a) would have been assigned to MAN in Condition 1 and the contralateral locus (INDEX 3 b) in Condition 4. In contrast, the disambiguating noun (BEARD) is inconsistent with the antecedent in Conditions 2 and 3. Here, the default would predict that the ipsilateral locus (INDEX 3 a) is assigned to WOMAN in Condition 2 and the contralateral locus (INDEX3b) in Condition 3.

Taken together, the prediction that signers automatically assign referents to R-loci based on the default principle implies that they are sensitive to the mismatch between the semantic continuation (BEARD) and the antecedent (WOMAN) referred to by INDEX in Conditions 2 and 3, relative to Conditions 1 and 4 (MAN). To test this hypothesis, we record participants' electroencephalogram (EEG) as they watch videos of signed sentences following the structure outlined above. We anticipate that participants' sensitivity to semantic mismatch will modulate three event-related potential (ERP) components, the Phonological Mismatch Negativity (PMN), the N400 and the P600.

The earliest component of interest is the PMN, which is thought to reflect phonological processing sensitive to expectations raised by the prior semantic context (Connolly \& Phillips, 1994; D’Arcy et al., 2000; Newman et al., 2003). For instance, Connolly \& Phillips (1994) report an early negativity (between $150-300 \mathrm{~ms}$ ) in response to sentence final words phonologically inconsistent with the semantic context thus far. This component typically occurs with a fronto-central distribution (Connolly et al., 1990, 1992) (but cf. Connolly \& Phillips, 1994). The N400 is a negative-going component with a broad distribution peaking between 200 and $500 \mathrm{~ms}$ following stimulus onset (for a review, see Kutas \& Federmeier, 2000, 2011). It indexes a complex of processes related to various aspects of meaning processing such as accessing and selecting conceptual representations 
from semantic memory and integrating them with existing contextual information (Lau et al., 2008). Its amplitude is modulated by the ease of semantic processing and increases when meaningful processing is hindered, such as, when a word is incongruent with the preceding semantic context. The P600 is a later positive-going component with a centro-parietal distribution peaking around $600 \mathrm{~ms}$ post-stimulus. While it was originally considered to index syntactic processing and reanalysis (Hagoort et al., 1993; Kaan et al., 2000; Münte et al., 1997), accumulating evidence highlights its sensitivity to semantic anomalies. Münte et al. (1998) suggest that the P600 indexes error monitoring and reanalysis. Vissers et al. (2006) and van de Meerendonk et al. (2011) interpret this component according to a monitoring theory of language perception van de Meerendonk et al. (2009), where comprehenders generate expectancies about upcoming information and monitor incoming information for errors. When linguistic input does not match the expected input, reanalysis is triggered due to mismatching representations. The strength of the conflict reflected in the amplitude varies with the degree of mismatch between expected and encountered events.

All three components have been investigated in sign language processing. For instance, Kutas et al. (1987) compared ERPs to sentences ending with a semantic violation for written and spoken English in hearing subjects and for ASL in deaf subjects. For each group, semantically anomalous sentences elicited an N400 effect with slight variations in the onset of the effect. The authors claim that underlying processing of semantic anomalies is similar in reading and listening to English and, crucially, processing of ASL. Similarly, Capek et al. (2009) presented deaf participants with signed ASL sentences containing either no violations, a semantic or a syntactic violation. Semantically anomalous signs elicited an N400 relative to baseline condition broadly distributed over posterior regions. Additionally, syntactic violations elicited a left anterior negativity followed by a P600. 
Further studies showed similar effects for DGS. Hänel-Faulhaber et al. (2014) investigated ERP patterns of signers in reaction to semantically or morphosyntactically anomalous sentences. Morphosyntactic violations evoked an early left anterior negativity followed by a broadly distributed positivity (P600), which can be seen as a typical biphasic pattern (similar to Capek et al. (2009)). Semantic violations, in contrast, elicited an N400 effect over fronto-central regions. Hosemann et al. (2013) presented participants with sentences ending with a semantically expected or unexpected sign. Unexpected signs evoked an N400 effect in line with Hänel-Faulhaber et al. (2014). However, this effect was found in the transition phase, i.e., the phase between two signs, suggesting that this phase already contains enough information to trigger detection of semantic anomalies even before the onset of the critical sign. Finally, regarding the PMN, while some studies suggest that this component may be restricted to the auditory modality (Capek et al., 2009; Gutiérrez, Williams, et al., 2012), Hosemann et al. (2013) suggest that their findings may also be compatible with early negativities to phonological mismatches with an expected sign, although they do not explicitly refer to this early negativity as a PMN.

Against this background, we predict that participants will show increased early negative deflections in brain activity to semantically mismatching signs in Conditions 2 and 3 , where the antecedent implicitly assigned to the R-locus (per default principle) conflicts with the continuing semantic information. Relative to that, Conditions 1 and 4, where the implicitly assigned antecedent is consistent with the continuing semantic information, will not show negative deflections. We predict this mismatch to trigger reanalysis of the preceding input indexed by a modulation of the P600 component with increased positivity in Conditions 2 and 3 relative to Conditions 1 and 4 . Such a pattern would provide strong support for the automatic assignment of referents to R-loci based on the default principle. 


\subsection{Methods}

\subsubsection{Participants}

Twenty-one deaf native signers of DGS from different regions of Germany (12 female, 9 male, age range: 20 - 51 years, mean: 33 years) participated in the experiment. Eighteen participants were congenitally deaf and 3 deafened later (between 16 and 36 months of age). One participant was excluded from the analysis due to excessive eye movement artifacts. All participants had deaf parents or exposure to DGS before the age of three, were right-handed (as verified by a handedness-test), had normal or corrected-to-normal vision and at least high school education level. Participants were volunteers, paid for their participation and tested in the Experimental Sign Language Lab at the University of Goettingen. All subjects gave written consent before the experiment. Ethics approval was provided by the Ethics Committee of the Institute of Psychology at the University of Goettingen.

\subsubsection{Material}

Forty experimental stimuli sets for each critical condition were developed resulting in 160 experimental stimuli (see Appendix B). Each stimulus set (as presented above) contained two natural sentences with no causal relation in keeping with the following structure.

The first sentence contained three signs in the neutral SOV word order. Two DRs were introduced without any kind of localization combined with a transitive non-localizing verb. As referents, we used animate entities, i.e., humans or domestic animals (Murmann, 2012), to ensure we had combinations where a) we could switch the order of referents without altering semantic and syntactic appropriateness of sentences, b) both referents could act as subject/agent, and c) both referents were equally salient. No compound signs or proper names, 
requiring either fingerspelling or a fixed name sign, were used because they could potentially increase processing load. Each referent occurred maximally ten times and each referent combination maximally twice. The verbs were transitive and did not localize referents in space. This excluded agreement verbs, which use the horizontal space for marking person and number agreement (Mathur \& Rathmann, 2012), and some plain verbs, which obligatorily require an additional person agreement marker (Rathmann, 2003). Additionally, the verbs chosen allowed switching the order of referents without impacting grammaticality or semantic appropriateness. Each verb was repeated maximally four times and each noun pair-verb combination only occurred once.

The second sentence contained three signs starting with an INDEX followed by two other signs. The INDEX always occurred in subject position but varied in its direction towards an R-locus. The INDEX functioned as a pronoun referring to the referent assigned to either the ipsilateral or contralateral locus, across conditions. The second sign, either an adjective, adverb, noun or possessive pronoun, was semantically neutral. The critical sign in sentencefinal position referred back to one of the two referents and was semantically incompatible with the other referent. Assuming that participants automatically apply the right-left default pattern, led to the four conditions described above. Note again that no referent was explicitly assigned to either of these loci in the first sentence, and the second sentence was designed to examine which referent that participants implicitly assigned to a particular R-locus was picked up by the INDEX.

The stimulus material was tested in a questionnaire study with 39 hearing native speakers of German (students at the University of Goettingen) to ensure that the second sentence exclusively refers to one of the previous referents. Here, participants were presented with 92 sentence sets (in written German) that contained a gap at the beginning of the second 
sentence. Participants were asked to fill the gap with a matching pronoun, to circle the corresponding referent and to rate the certainty of their pronoun choice. 18 participants were excluded from the analysis because they were not native speakers, filling in the questionnaire twice or not completing the task correctly. We chose 40 sentence sets that showed the clearest referent choice and highest certainty ratings as final stimulus sets.

For the actual study, stimuli were interspersed with 80 filler sentences. The material was recorded on video with two male deaf native signers of DGS using a Sony HDRCX550VE camcorder capturing frontal view. We controlled for the usage of non-manuals to exclude any kind of overt spatial localization such as eye-gaze, body-movements, head-nods or eyebrow raise since not much is known about the influence of non-manuals on anaphora processing in sign languages. However, lexical non-manuals, including mouthing, were allowed because these serve as an important lexical disambiguation device in DGS (Ebbinghaus \& Heßmann, 2001). There was a natural prosodic break between the two sentences of $108 \mathrm{~ms}$ on average (40 ms - $400 \mathrm{~ms}$; no significant differences across conditions) and all verbs were signed in the neutral space in front of the signer.

Videos were cut and processed with Adobe Premiere Pro CS6. The signer remained motionless for $2000 \mathrm{~ms}$ at the beginning and $1500 \mathrm{~ms}$ at the end of each video. Videos had a size of $1280 \times 720$ pixels and a duration of $7000 \mathrm{~ms}$ to $9320 \mathrm{~ms}$ (mean: $8550 \mathrm{~ms}$ ). Video duration did not differ significantly across groups (Condition 1: $8533 \mathrm{~ms}$, Condition 2: 8574 ms, Condition 3: $8544 \mathrm{~ms}$, Condition 4: $8548 \mathrm{~ms})$.

Additionally, a set of six practice items was developed with four items similar to the experimental stimuli and two being similar to the filler sentences. The sign combinations were 
not used during the experiment. They were recorded the same way as sentences in the experiment.

\subsubsection{Pretest and Posttest}

Additional tests were conducted to ensure that participants knew the crucial signs of the stimuli given possible regional variations of DGS (Eichmann \& Rosenstock, 2014; Langer, 2012). In a pretest, referents from all four conditions used in the first sentence were presented as single signs with their corresponding meaning (German word) as a subtitle. Participants were asked to indicate via button press whether they relate the sign to the presented meaning. In a posttest, participants were asked to write down the meaning of the final signs of the second sentence presented as videos of individual signs in isolation. Since the analysis of ERPs was triggered to this sign, understanding of these signs was checked following the experiment to exclude modulations of the data. Familiarity of each participant with all items and familiarity of each item to participants were evaluated. Participants on average knew $97 \%$ of the items (range: $74-100 \%$ ) in the pretest and on average $97 \%$ (range: $84-100 \%$ ) in the posttest. Items were familiar to participants on average $97 \%$ (range: $76-100 \%$ ) in pretest and on average $95 \%$ (range: $71-100 \%$ ). Thus, no participant and no item were excluded from the analysis based on both tests.

\subsubsection{Procedure}

Testing took place in the Experimental Sign Language Lab at the University of Goettingen with the help of a native DGS signer to ensure a DGS language setting. Participants were first informed about the procedure and the EEG technique. All explanations were given in written form and sign language to ensure that all subjects understood the 
provided information. Then, participants gave their signed consent, filled in a metadata form and participated in a handedness test (www.sign-lang.uni-hamburg.de/dgs-korpus).

Participants then took part in the pretest, followed by the experimental session and finally, the posttest.

The experiment took place in a top-lit cabin. Participants were seated on a chair in front of a 24-inch screen at approximately $1 \mathrm{~m}$ distance from the screen. Stimuli were presented using the experimental software Presentation, Version 18.1 (Neurobehavioral Systems, Inc.). At the beginning, an introduction video in DGS explained the procedure followed by a practice session to familiarize participants with the trial structure (Figure 1.2). Participants could repeat the practice session if required. This was followed by the actual experiment. Each trial began with a blank screen for $500 \mathrm{~ms}$ followed by a video, displayed in the center of the screen, showing one sentence set in the speed of natural signing. At the end of each video, a question mark appeared indicating that participants should judge whether the sentence was good or bad by relying on their first immediate reaction and by pressing either a green 'thumbs up' button or a red ‘thumbs down' button on a Microsoft Xbox 360 Wired Controller. There was no time limit for their response. Participants were instructed to avoid body movement and, if possible, to blink after the video was presented. Between blocks, participants could take breaks and could decide themselves when to continue with the experiment. 


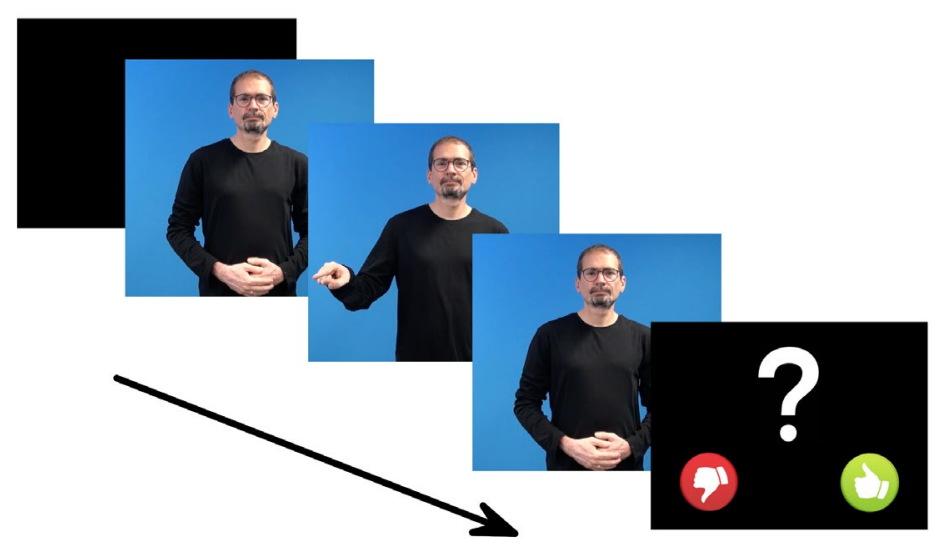

Figure 1.2 Structure of a single trial

Experimental sentence sets were combined into four different lists, pseudo-

randomized in 10 blocks of 24 sentence sets each. Each block contained eight filler sentences and 16 experimental stimuli with four sentences from each condition, ensuring that sentences within a block were all from different sentence sets. The order within a block was randomized on-line.

\subsubsection{EEG-recording}

We recorded $\mathrm{EEG}$ data using 64 active $\mathrm{Ag} / \mathrm{AgCl}$ electrodes placed according to the international 10-20 system and data were amplified using an ActiveTwo AD-Box (BioSemi B.V., Amsterdam, Netherlands) with a sampling rate of 2048 Hz. Data were re-referenced offline to linked mastoids. Impedances were kept below $20 \mathrm{k} \Omega$. For each participant, the electrooculogram was monitored using three external electrodes, two of which recorded the horizontal and one recorded the vertical electrooculogram in combination with electrode FP2 on the cap. EEG data were analyzed using the ERPLAB toolbox plugin (Lopez-Calderon \& Luck, 2014) for MATLAB (The MathWorks, Inc.). The EEG signal was filtered using a 0.1$30 \mathrm{~Hz}$ band-pass filter and downsampled to $250 \mathrm{~Hz}$. We calculated ERP averages for single 
subjects for each condition and electrode at different trigger points. Hosemann et al. (2013) revealed that processing of signs is triggered even before the sign's onset is presented (similar to co-articulation information in spoken language). Therefore, we defined three different triggers following Hosemann et al. (2013).

1. Sign offset: Last frame of the final hold of the sign preceding the critical sign before the hand gets relaxed again and before the movement of the transition phase starts. This trigger was used for baseline correction.

2. Target handshape: First frame in which the target handshape was identifiable, regardless of target orientation or location. On average, the target handshape trigger position occurred $146 \mathrm{~ms}$ after the sign offset2 trigger position.

3. Sign onset: First frame of the critical sign where the target hand configuration reached its target location and the hands were about to start the path movement. On average, the sign onset trigger position occurred $202 \mathrm{~ms}$ after the target handshape trigger position. The duration of the critical sign, defined as duration from sign onset to sign offset, was on average $384 \mathrm{~ms}$.

These trigger positions were manually identified by two independent coders for each video using the ELAN software (Wittenburg et al., 2006). ERPs were timelocked separately to target handshape and sign onset triggers and corrected for baseline activity $200 \mathrm{~ms}$ prior to sign offset triggers of the final disambiguating sign in the second sentence. Trials with blinks or other artifacts were rejected before averaging with an artifact rejection threshold of 100 $\mu \mathrm{V}$. Preliminary analyses run on $100 \mathrm{~ms}$ epochs across trials revealed significant differences between conditions following the target handshape trigger between $200-300 \mathrm{~ms}$ and $500-$ 
$600 \mathrm{~ms}$ in keeping with the early PMN and N400 time windows. However, no time window associated with the P600 revealed significant differences. We, therefore, focused on the two significant time windows for further analyses. Additional analyses on time windows following the sign onset trigger revealed no significant differences. All EEG and behavioral datasets underlying the analysis in the current study are available using the following DOI: 10.17605/OSF.IO/NBMRJ deposited in the Open Science Framework.

\subsection{Results}

\subsubsection{Behavioral data}

Nineteen participants were included in the judgement data analysis (one participant was excluded as described in the methods section and one participant was excluded due to technical problems). Mean judgement ratings obtained from participants for each condition are reported in Table 1.1. There were no differences in participants' judgements of sentence acceptability across conditions $(p>.1)$.

Table 1.1. Mean judgement ratings and standard deviations for each condition.

\begin{tabular}{ccc}
\hline Condition & Mean & Standard deviation \\
\hline $\mathbf{1}$ & 1.45 & 0.31 \\
$\mathbf{2}$ & 1.45 & 0.32 \\
$\mathbf{3}$ & 1.47 & 0.31 \\
$\mathbf{4}$ & 1.39 & 0.31 \\
\hline
\end{tabular}

For means, 1 indicates a rating as 'good' and 2 indicates a rating as 'bad'. 


\subsubsection{ERP data}

For evaluation, we analyzed the identified time windows, i.e., $200-300 \mathrm{~ms}$ and $500-$ $600 \mathrm{~ms}$, in two different ways. In the following, only significant and near-significant results are reported.

First, data from Condition 1 with 4 and Conditions 2 with 3 were combined to examine whether participants automatically assigned the first referent to the ipsilateral locus. This would render the referent congruent with the disambiguating sign in Conditions 1 and 4, and incongruent in Conditions 2 and 3, thus focusing on congruence or incongruence between disambiguating sign and referent assigned to the locus regardless of what this locus (INDEX3a / INDEX 36 ) is.

Data from lateral electrodes (F1-8, C/T1-8, P1-8,) were split by region and hemisphere, and analyzed with a $4 \times 3 \times 2 \times 2$ ANOVA with the factors laterality (far-left, left, right, far-right), region (frontal, central, parietal), hemisphere (left, right) and match (congruent, incongruent). The Greenhouse-Geisser correction (Greenhouse \& Geisser, 1959) was applied as required. Visual data inspection suggested a sustained negativity across the two conditions, with increased negative activity to incongruent relative to congruent conditions (Figure 1.3). However, analysis across these conditions yielded no significant differences in the early time window $(200-300 \mathrm{~ms})$. 

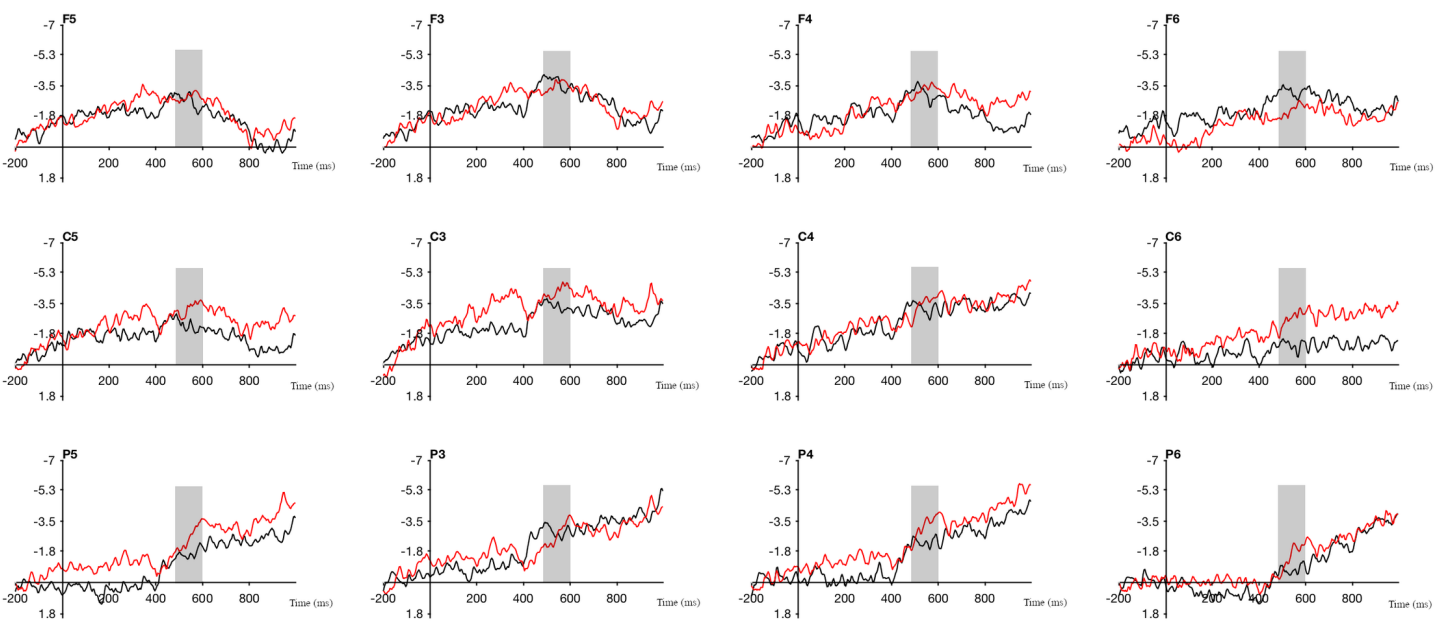

Figure 1.3 Grand average ERPs for congruent (black) and incongruent (red) conditions. Data are timelocked to the target handshape trigger. $\mathrm{C} 3$ and $\mathrm{C} 5$ are central right and left lateral electrodes on the left hemisphere. C4 and C6 are central left and right lateral electrodes on the right hemisphere. Negativity is plotted upwards.

Analysis of the later time window, from 500 - $600 \mathrm{~ms}$, revealed a significant four-way interaction $(\mathrm{F}(6,114)=3.041 ; p=.009)$, an interaction between hemisphere*laterality*match $(\mathrm{F}(3,57)=3.099 ; p=.034)$ and a near-significant interaction between region*match $(\mathrm{F}(2,38)$ $=3.554 ; p=.055)$. Pivoting on laterality revealed a significant three-way interaction between region*hemisphere*match in the data across left $(\mathrm{F}(2,38)=5.461 ; p=.008)$ and right electrode sites $(\mathrm{F}(2,38)=3.490 ; p=.041)$ and a near-significant interaction between region*match across far right electrodes $(\mathrm{F}(2,38)=2.974 ; p=.063)$.

Pivoting on hemisphere, analysis of left hemisphere left electrode sites with the factors region and match revealed a near-significant interaction $(\mathrm{F}(2,38)=3.462 ; p=.056)$, which we broke down to reveal a near-significant difference between congruent $(M=-2.798)$ and incongruent $(\mathrm{M}=-4.782)$ conditions across central electrode sites $(\mathrm{t}(19)=1.998 ; p=.06)$. No 
significant differences between conditions were found for right hemisphere left electrode sites.

Analysis of right hemisphere right electrode sites with the factors region and match revealed a significant interaction $(\mathrm{F}(2,38)=5.992 ; p=.005)$, which revealed a significant difference between congruent $(M=-0.945)$ and incongruent $(M=-2.979)$ conditions across the central region $(\mathrm{t}(19)=2.364 ; p=.029)$.

A second analysis split conditions depending on whether the locus picked up by the INDEX was the ipsilateral or contralateral locus, i.e., separately compared Conditions 1 to 4 , since participants may expect the first referent to be referred to in the second sentence relative to the second referent. This would render Condition 1 more appropriate than Condition 4. Data from lateral electrodes (F1-8, C/T1-8, P1-8,) were split by region and hemisphere and analyzed with a $4 \times 3 \times 2 \times 2 \times 2$ ANOVA with the factors laterality (far-left, left, right, far-right), region (frontal, central, parietal), hemisphere (left, right), direction (ipsilateral, contralateral) and match (congruent, incongruent).

Across the early time window, from 200 - $300 \mathrm{~ms}$, analysis revealed a four-way interaction of hemisphere*region*laterality*match $(\mathrm{F}(6,114)=2.797 ; p=.014)$, a four-way interaction of hemisphere* region*direction*match $(\mathrm{F}(2,38)=3.956 ; p=.028)$ and a four-way interaction of hemisphere*laterality*direction*match $(\mathrm{F}(3,57)=3.582 ; p=.019)$. It also revealed three-way interactions between hemisphere*region*direction $(\mathrm{F}(2,38)=3.468 ; p=$ $.041)$ and hemisphere*laterality*direction $(\mathrm{F}(3,57)=3.943 ; p=.033)$ as well as interactions between hemisphere*direction $(\mathrm{F}(1,19)=5.325 ; p=.032)$ and region*match $(\mathrm{F}(2,38)=$ $6.198 ; p=.014$ ). Pivoting on region (Figure 1.4), paired samples t-tests comparing congruent $(\mathrm{M}=-0.579)$ and incongruent $(\mathrm{M}=-1.120)$ conditions revealed a near-significant difference 
across central regions $(\mathrm{t}(19)=1.891 ; p=.074)$ and a significant difference between congruent $(\mathrm{M}=0.127)$ and incongruent $(\mathrm{M}=-0.618)$ conditions across parietal electrodes $(\mathrm{t}(19)=$ $2.322 ; p=.032)$. This mirrors the findings in the analyses reported above with overall increased negativity to incongruent relative to congruent trials across parietal and central regions, also in the early time window.
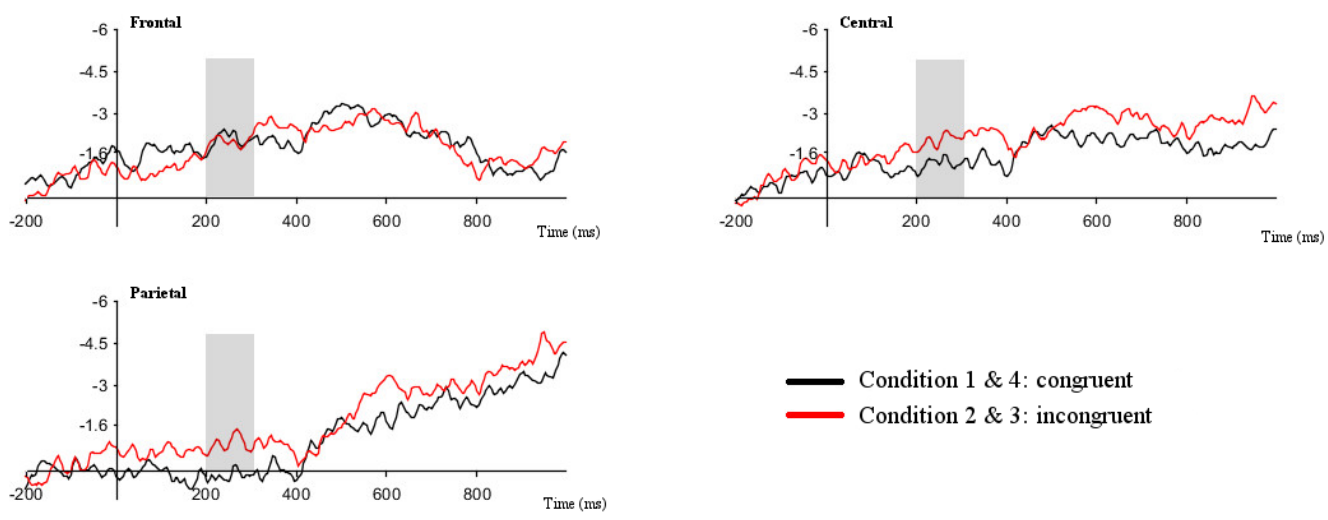

Figure 1.4 Grand average ERPs congruent (black) and incongruent (red) conditions. Data are timelocked to the target handshape trigger for frontal, central and parietal regions across left and right hemisphere. Negativity is plotted upwards.

Breaking down the interaction between hemisphere and direction (Figure 1.5), we found increased negative activity in the left relative to the right hemisphere when the INDEX picked up the contralateral locus $(\mathrm{t}(19)=-4.517 ; p=.000)$. This suggests increased processing costs in the left hemisphere when the contralateral direction is processed. In the time window from $500-600 \mathrm{~ms}$, a similar effect was found when the data were grouped by direction regardless of match with increased negative activity across left hemisphere sites relative to right hemisphere sites when the INDEX pointed to the contralateral locus, $(\mathrm{t}(19)=$ $2.362 ; p=.029)$. No other comparisons yielded significance. 

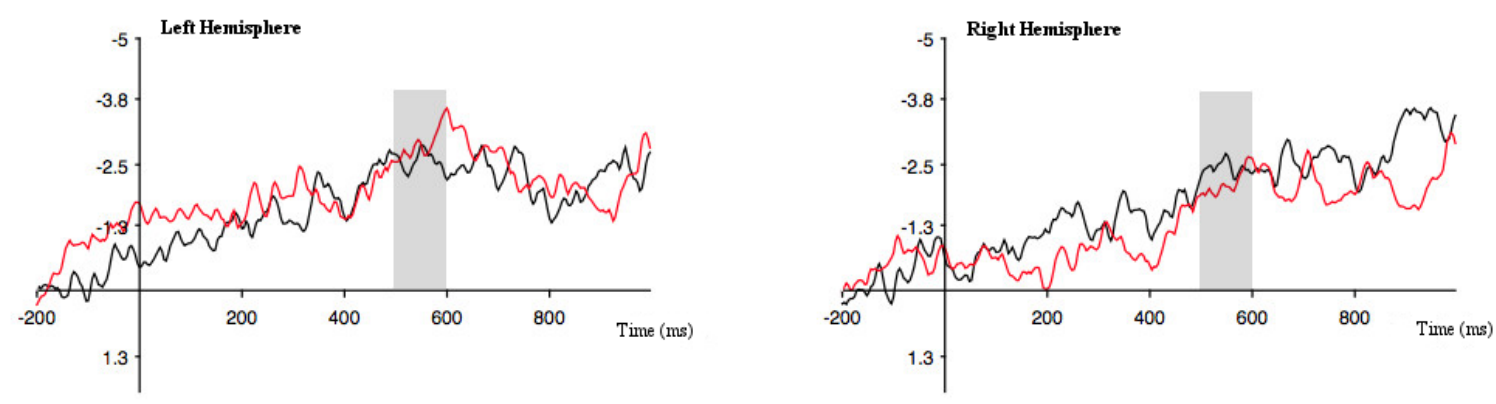

- Condition $1 \& 3$ : ipsilateral (right) INDEX sign

- Condition 2 \& 4: contralateral (left) INDEX sign

Figure 1.5 Grand average ERPs for conditions containing an ipsilateral/right (black) and contralateral/left (red) index. Data are timelocked to the target handshape trigger for both left and right hemisphere. Negativity is plotted upwards.

\subsection{Discussion}

This study investigated to which extent signers employ the right-left default pattern governing the assignment of DRs to R-loci in the horizontal plane in DGS. We examined this by presenting participants with sentences assigning referents implicitly to an R-locus, and a pronoun picking up one referent either consistent or inconsistent with a sentence-final semantically disambiguating sign. Increased negative deflections in the N400 time window indicated that participants indeed applied the proposed pattern of referent assignment, thus confirming our hypotheses. First of all, the influence of congruence of the disambiguating term and antecedent implicitly assigned to a locus provides evidence that signers automatically assign distinct and contrastive R-loci to different DRs, since the antecedent referred to was never explicitly assigned to this locus. Second, our results suggest that antecedents are not arbitrarily assigned, but rather, that there appears to be a specific default 
governing the assignment to distinct R-loci. In particular, the results can only be explained by suggesting that right-handed signers indeed apply the right-left default pattern implemented in a modified version of Discourse Representation Theory as proposed by Steinbach \& Onea (2016). Thus, the study reveals that, even in the absence of an overt localization strategy, signers construct a spatial structure of the present discourse to guide their understanding of referential expressions. They automatically make use of opposing R-loci in the horizontal plane towards, in this case, anaphoric disambiguation. Therefore, R-loci can be analyzed as a modality-specific grammatical device that is used to structure the set of DRs in the DRS and to restrict the set of possible DRs that a pronominal expression is referring to. Hence, the results provide evidence that DGS exploits the specific properties of the signing space to facilitate reference tracking in signed discourse.

This allows for an interesting parallel between spoken languages like German and DGS, since grammatical gender fulfills a similar function in the processing of many spoken languages. In German, for instance, nouns take one of three genders, i.e., masculine, feminine and neuter. Pronouns agree in grammatical gender with antecedents they refer to (e.g., der/er for masculine nouns). In a discourse containing a masculine and a feminine noun, a masculine pronoun unambiguously refers back to the former and speeds up activation of the correct antecedent even before further information is provided (for a review see Friederici \& Jacobsen (1999)). This is similar to the role of R-loci in sign language processing since referring to an R-locus retrieves the antecedent assigned to it, thereby facilitating further processing of the discourse. Thus, sign and (some) spoken languages appear to have both developed similar but modality-specific strategies to fulfill the same discourse function.

Additional analyses examined differences between trials within conditions to determine whether participants expect the first referent to be continued with in subsequent 
discourse. This revealed three interesting findings. First, we found no modulation of ERPs by direction alone within either congruent or incongruent trials. While this might be taken as evidence that participants have no preference for the first DR, we suggest caution in reaching this interpretation until this finding has been replicated in sentences containing referents explicitly assigned to particular loci - it may be that implicitly assigned R-loci are too weak to support such pragmatic constraints on discourse interpretation. Second, we found a modulation of ERPs by congruence of disambiguating term and antecedent implicitly assigned to the R-locus in the early time window. Here, semantic/phonological expectations are violated, thus the modulation reflects participants' sensitivity. Third, we found a lateralization effect with increased negative deflections in the left hemisphere for the contralateral INDEX, i.e., the left side of the signer (right visual field of participants). We interpret this finding in keeping with reported intrinsic attentional biases of the left hemisphere to the contralateral visual field, with earlier latencies and enhanced amplitude contralaterally for early ERP components (e.g., N2 and P2; Andreassi et al., 1975; Rugg et al., 1985), rather than effects of violation of phonological expectation, as in the case of the PMN. Nevertheless, this early time window is modulated by congruence of disambiguating sign and antecedent picked up by the R-locus (regardless of the direction of this locus). This suggests different contributors to the modulation of ERPs in this time window, one sensitive to visual field attentional biases and the other sensitive to violations of semantic/phonological expectations. The latter modulation resembles the PMN effect which has, to date, not been reported for sign language processing. Further research is needed to examine the different processes contributing to differences in this early time window and to which extent the PMN is shared across modalities.

Similar to other ASL studies (e.g., Gutiérrez et al., 2012) the current N400 component seems to peak later than usual (cf. Kutas \& Federmeier, 2011). Capek et al. (2009) argue that 
the onset of this effect resembles findings observed for written stimuli. The N400 component is usually observed between 300 and $500 \mathrm{~ms}$ (Kutas \& Federmeier, 2011). However, the N400 effect in the current study occurred in a different time window, later than usual (500 $600 \mathrm{~ms}$ ). A closer look at previous studies on ASL reveals that they show a similarly delayed N400. Gutiérrez et al. (2012) observe an N400 between 450 - 600 ms and Capek et al. (2009) similarly report effects of semantic processing in a broad time window from 300 to $875 \mathrm{~ms}$. The authors state that the onset of this effect resembles findings observed for written stimuli. Indeed, effects for auditory conveyed information seem to occur earlier than similar effects evoked by visual information irrespective of whether they examine processing of written or signed stimuli (Holcomb et al., 1992; Kutas et al., 1987; Kutas \& Federmeier, 2011). Thus, a delayed onset of the N400 might be typical for sign languages due to their modality.

Another potential reason for the delayed N400 may be related to the delay between the onset of our analysis window and the uniqueness point of a sign, i.e., the point when this sign can be recognized. The analyses reported in 3.2 are timelocked to the target handshape trigger, i.e., the first frame in which the target handshape was identifiable. This occurred on average $200 \mathrm{~ms}$ before sign onset, i.e., first frame in which the target hand configuration reached the target location and the hands started their path movement. Variability regarding when the sign is recognizable and when processing is advanced enough to trigger a match or a mismatch response, might cause the delay. This might suggest that our current analysis is timelocked to an earlier trigger relative to when detection of a mismatch is possible. However, there are no standardized regulations to determine trigger positions in EEG experiments investigating sign languages. Indeed, Hosemann et al. (2013) provided evidence for the important role of the transition phase between two signs for EEG studies. Transition phases are longer and more pronounced in sign languages since they use the signing space to convey information, which results in longer transitions from one sign to the next due to their spatial 
distribution and relatively large, slow and heavy articulators (Meier, 2002). Additionally, transitions between signs have different lengths and trajectories that depend on the context and surrounding signs. Therefore, information is available at different time points for each transition and it is hard to determine a universally identifiable trigger position, leading to issues with regard to time locking electrical activity to particular time points in the signal.

An additional point to be considered is the language status of the participants. While all participants were native signers, none of them were monolinguals in the strict sense. Participants are surrounded by written German and thus acquire German as a second language early in life. The bilingual background may influence the timing of expected effects. A number of studies suggest that the N400 effect in bilinguals, while showing a similar distribution, can vary in both amplitude and latency (Ardal et al., 1990; McLaughlin et al., 2004; Midgley et al., 2009). Indeed, in a cross-modal priming study on DGS, Hosemann (2015) found that native signers automatically activate the German counterpart of a DGS sign. Such unconscious cross-language cross-modal activation may modulate both behavioral data - leading to slower reaction times - and ERP data - leading to changes with regard to amplitude and latency of particular components (Kubus et al., 2015; Thierry \& Wu, 2007).

Moreover, it is worth discussing the absence of any modulation of the P600 component. As outlined in 1.4, one might expect a similar modulation of the P600 given the semantic anomaly between disambiguating sign and referent. Alternatively, one might expect syntactic reanalysis regarding referent assignment triggered by this disambiguating sign. We suggest two reasons for the absence of such a modulation. First, it is possible that processes of error monitoring abort at some point, due to increased semantic implausibility. This possibility is refuted, however, by studies suggesting that even implausible sentences trigger error monitoring (e.g., van Herten et al., 2006). Second, the analysis of the behavioural data 
showed that sentences were equally acceptable with no significant differences in judgement ratings for any condition. This would suggest that sentences are, from a syntactic perspective, at least, well-formed and would, therefore, not trigger a P600 effect. Indeed, our finding of a modulation of semantic and not syntactic ERP indices supports this suggestion. This would reflect the strength of the default assignment, that possibly can be overwritten easily once the semantic anomaly is detected, mirrored in ERP components modulation.

Finally, it might be surprising that in the behavioural data all conditions were rated similarly even if conditions 2 and 3 were expected to receive lower ratings due to their semantic mismatch compared to conditions 1 and 4 . One reason for the similar ratings may be the high variability and the lack of a standardized form of DGS. Thus, participants may have a higher tolerance to the provided language input. Moreover, the language status of participants as possible bilinguals as mentioned above and thus the influence of spoken German might affect their ratings (Hosemann et al., 2018). Lastly, the lack of a difference in the ratings may be due to a pragmatic effect related to anaphora resolution. The behavioural task follows stimulus presentation and thus a (late) context-driven re-interpretation could apply to achieve a successful interpretation of the sentence set. Additional pragmatic factors such as accessibility may apply here as well which requires more evidence from future research.

\subsection{Conclusion}

By investigating the modality-specific way of introducing DRs and establishing anaphoric relations in DGS, this experimental study improves our understanding of discourse structures in sign languages, it provides new insights into the impact of modality on linguistic structure in particular and on the structure of the human language faculty in general. The present study demonstrated that a) right-handed deaf native signers of DGS assign distinct 
and contrastive R-loci to different DRs and b) that a specific default pattern governs the Rloci assignment of antecedents. In particular, right-handed signers implicitly assign the first referent to the ipsilateral and the second referent to the contralateral side of the signing space. This suggests that signers employ a specific default strategy for anaphora resolution (space) similar to spoken language users (gender). Such defaults can, however, be overwritten by other factors influencing discourse structuring, such as overt localization, information structural issues, and well-formedness as indicated by participants' judgements. It remains an open question whether the observed default pattern holds for left-handed signers as well. A difference in the observed pattern would suggest that the location of both sides and terms would be defined in relation to the dominant hand of the signer in line with Geraci (2014). Moreover, the pattern may depend on the handedness of the signer presented in the stimulus material. Ongoing research in our sign language lab is currently assessing this and other factors influencing the processing of DRs in sign language research. 


\title{
2 Processing pronominal pointing signs in German Sign Language: Neurophysiological evidence for the first mention effect
}

\begin{abstract}
While the first mention bias has been well investigated in spoken languages, less is known about the presence of a similar bias in sign languages. In sign languages, pronominal pointing signs are directed towards referential locations in the ipsilateral and contralateral area of the signing space associated with referents in previous contexts. The present event-related potential study examined the presence of a first mention effect during pronoun resolution in German Sign Language (DGS). We presented participants with sentence sets containing two referents in the first sentence and a pronominal sign at the beginning of the second sentence directed to either the ipsilateral or contralateral area in space. Results show an N400 for contralateral compared to ipsilateral pronominal signs suggesting increased processing costs associated with the second referent assigned to the contralateral area. Thus, the current study provides evidence for a first mention effect in DGS highlighting its modality independent nature.
\end{abstract}

Keywords: German Sign Language, event-related potentials, first mention effect, N400 


\subsection{Introduction}

To process and comprehend an anaphoric pronoun, individuals are required to establish a co-reference relation between the pronoun and its corresponding antecedent. One way to establish co-referential relations during the anaphora resolution process in spoken languages is to rely on grammatical markers such as gender (Arnold et al., 2000). In unambiguous cases, the antecedent can be identified easily and the co-reference relation is resolved. Cross-linguistically, a number of different factors guide pronoun resolution in ambiguous contexts, including the so-called first mention effect, according to which ambiguous pronouns are interpreted as co-referential with the referent mentioned first in the discourse (Gernsbacher et al., 1989; Von Eckardt \& Potter, 1985).

Unlike spoken languages, sign languages make use of the linguistic potential of the three-dimensional signing space to guide referential relations. Referents are assigned to specific areas in the signing space. Directing a pronoun to that area in the subsequent discourse establishes a co-referential relation between the pronoun and the corresponding referent assigned to this area. A few studies investigating pronoun resolution preceded by overtly localized referents examine a first mention effect in sign languages as well (Emmorey \& Lillo-Martin, 1995; Krebs et al., 2018; Krebs, Malaia, et al., 2019). However, in sign languages, referents do not have to be overtly localized in space. This may lead to supposedly ambiguous situations resulting in miscommunication, but this is not the case and communication happens successfully. One explanation might be the first mention bias applied by the addressee to resolve ambiguous contexts. Against this background, the current study investigates whether there is a first mention effect in German Sign Language in contexts where antecedents have not been overtly localized in the signing space. We briefly review previous literature related to the first mention effect in spoken languages and give a brief 
overview on pronoun resolution strategies in sign languages before presenting the current study.

\subsubsection{First mention effects in spoken languages}

Previous experimental studies have observed the first mention effect across numerous languages. The first set of studies reporting this effect focused on English sentence pairs across a variety of different tasks. Using a probe recognition task, Gernsbacher \& Hargreaves (1988) examine the impact of linguistic factors, i.e., theta roles, sentence-initial position and syntactic subjecthood, on pronoun resolution. The data reveal a first mention effect reflected in faster reaction times to probes referring to the first mentioned referent than to probes referring to the second mentioned referent over and above the other linguistic factors investigated. Similarly, in a sentence completion task, Crawley \& Stevenson (1990) present participants with sentence fragments that either end with a pronoun or with a conjunction. In both comprehension and production tasks, participants prefer the first mentioned referent as the antecedent of the referential expressions, i.e., pronouns, independent of the sentence fragment's presentation in isolation or in discourse context. This preference is stronger in comprehension than in production. Similar effects are observed by Crawley et al. (1990) in a reading and an assignment task showing that pronouns in object position within the target sentence show a strong preference towards a preceding subject, i.e., the first mentioned referent, but only if pronouns were ambiguous and no gender cue was available. Extending this to Spanish, Carreiras et al. (1995) use a probe recognition task to examine the first mention preference in Spanish sentences, which allow greater flexibility in word order relative to English sentences. In a series of experiments, they present data showing a first mention preference in Spanish, which is reflected in faster reaction times to first mentioned referents irrespective of their syntactic status and the animacy of the arguments. 
Other studies describe a similar effect, the subject preference, which identifies the antecedent of an ambiguous pronoun as the grammatical subject of the previous sentence (Bornkessel-Schlesewsky \& Schlesewsky, 2009). In a grammaticality judgement task on Dutch declarative sentences, Frazier \& d'Arcais (1989) observe that participants produce less errors and respond faster to subject-initial trials compared to object-initial trials, which is explained as reflecting a subject preference. For German relative clauses, Schriefers et al. (1995) show, in a self-paced reading task, that relative clauses with an ambiguous sentenceinitial relative pronoun are preferentially interpreted as subject relative clauses under neutral conditions, i.e., no biased disambiguating element preceding the relative pronoun, reflected in shorter reading times to interpretations in keeping with this preference.

For languages such as English, in which the grammatical subject is typically also the first mentioned referent, it is difficult to disentangle the first mention effect from the subject preference effect. Järvikivi et al. (2005) aim to disentangle these phenomena based on order of mention and on grammatical role in the processing of ambiguous pronouns. For this purpose, they examine the processing of pronouns in Finnish, a language with free word order and a gender neutral third person singular pronoun. The results show that subject and first mention preference both come into play in the resolution of ambiguous pronouns although the subject preference results in a stronger effect than the first mention preference.

\subsubsection{Pronoun resolution strategies in sign languages}

Sign languages exploit the signing space located in front of the signers' head and upper part of the body, to express various phonological, morphosyntactic, semantic and pragmatic functions (P. Perniss, 2012). The signing space is divided into different planes, 
among which, the horizontal plane plays an important role in indicating role shift (LilloMartin, 2012), temporal reference and topographic relations (Emmorey, 2002), plurals (Pfau \& Steinbach, 2006), contrast (van der Kooij et al., 2006), agreement (Padden, 1990) and, crucial for the current study, the spatial localization of referents (Lillo-Martin \& Klima, 1990).

In sign languages, pronouns are expressed using pointing signs that are typically produced with an extended index finger directed towards a specific area in the signing space. The pointing sign (INDEX sign) is used to express various grammatical functions, such as determiners, locative adverbials or personal pronouns (Cormier et al., 2013; Pfau, 2011). In their pronominal function, pointing signs can either refer deictically to the signer, the addressee and third person referents physically present in the actual discourse, or anaphorically to third person referents not physically present in the actual discourse (for a review, see Cormier (2012)). Referring to physically present third person referents is easily achieved by pointing to their actual location in the communicative situation. In this case, the relation between the pronoun and its antecedent is established via deictic reference. However, in case a third person referent is not physically present, this antecedent has to be located on the horizontal plane of the signing space to establish an anaphoric relation between the antecedent and the corresponding pronoun. Therefore, a referent is assigned to an arbitrary locus in the horizontal plane, a referential locus (henceforth, R-locus) (Liddell, 1990; LilloMartin \& Klima, 1990; Sandler \& Lillo-Martin, 2006). Subsequently, a pronominal (pointing) sign can be directed towards that locus to pick up the established referent (Lillo-Martin \& Klima, 1990; Sandler \& Lillo-Martin, 2006).

Assignment to an R-locus is typically accomplished by a combination of manual, e.g., pointing signs (INDEX sign) or person agreement markers among others (Liddell, 1990; Pfau 
\& Steinbach, 2013), and nonmanual strategies, i.e., body leans, head movements or eye gaze towards the respective R-locus (Lillo-Martin, 1986; Winston, 1996). Yet, these strategies can also be used separately. Establishing R-loci in the horizontal plane appears to follow a particular pattern in keeping with the order of introduction of the referents, i.e., first and second mentioned, and directions, i.e., ipsi- and contralateral. A typical overt establishment of referents for right-handed signers of German Sign Language (DGS) involves the assignment of the first mentioned referent to the right (ipsilateral) and the second referent to the left (contralateral) side of the signing space (Steinbach \& Onea, 2016). A similar pattern has been described for Catalan Sign Language (Barberà, 2012), Italian Sign Language (Geraci, 2014), American Sign Language (ASL) (Sandler \& Lillo-Martin, 2006; Schlenker, 2013) and Israeli Sign Language (Meir \& Sandler, 2008). Studies on British Sign Language (Sutton-Spence \& Woll, 1999) and Danish Sign Language (Engberg-Pedersen, 1993) only mention the use of contrasting referents when using ipsi- and contralateral sides of the signing space, but do not elaborate on a specific order using these locations.

However, in cases where referents are not assigned overtly to an R-locus, one strategy signers rely on is a default pattern of referent localization in anaphora resolution. This default appears to follow the same strategy described for overt localization: first mentioned referents are assigned to the right/ipsilateral area and second mentioned referents to the left/contralateral side of the horizontal plane. This assumption is supported by empirical evidence reported in (Wienholz, Nuhbalaoglu, Mani, et al., 2018). In their study, Wienholz et al. (2018) present participants with short strings of discourse consisting of two sentences while recording their electroencephalogram (EEG). The first sentence introduces two referents without overtly localizing them in space and the second sentence always starts with a pronominal pointing sign either directed to the right/ipsilateral or left/contralateral side of the horizontal plane followed by a neutral sign and a sentence-final sign that is semantically 
biased towards one of the referents in the first sentence. The data show an N400 effect for conditions containing a semantic mismatch, i.e., when the pronoun picked up the referent localized on the right side in the signing space while the sentence final sign in the second sentence referred to the referent established by default on the left side in space or vice versa. Thus, signers implicitly apply the default pattern leading to the observed effect. The study, therefore, provides experimental evidence for the assumption that the observed pattern for overt localization is a default pattern that is also applied in cases of covert localization in DGS.

\subsubsection{First mention effects and subject preference in sign languages}

In sign language research, both a first mention effect and a subject preference have been investigated in a few previous studies. ${ }^{5}$ Emmorey \& Lillo-Martin (1995) present participants with signed sentences of ASL containing two referents followed by a second sentence containing either an overt pronoun co-referential with one of the previously introduced referents, a null pronoun licensed by verb agreement or no anaphora (control condition). They then measure reaction times for all conditions to probe signs representing one of the referents introduced in the first sentence. Responses in both pronoun conditions are faster than in the control condition suggesting that overt and null pronouns both reactivate their antecedents. Importantly for the current study, they find that reaction times to subject and object probe signs do not differ in the null pronoun condition leading the authors to argue that there is no first mention bias for ASL pronouns. They suggest that the absence of such an

\footnotetext{
${ }^{5}$ Recent work using offline methods such as production tasks show a second mention effect in different sign languages (Frederiksen, 2019; Nuhbalaoglu, 2018). However, since the current study uses online methods, a direct comparison of the results of these studies is not appropriate and will not be made here.
} 
effect might be due to the phonological properties of verb morphology leading to simultaneous identification of subject and object or that the effect may only apply to explicitly expressed noun phrases and not to pronouns in general. However, Emmorey \& Lillo-Martin (1995) could only claim the absence of this effect for the null pronoun condition. Their claims ought not to be generalized to conditions including overt pronouns, since they do not present analyses of reaction times to probe signs that are either the first or second mentioned referent in the overt pronoun condition. Only such an analysis has the potential to reveal whether there is a first mention effect for overt pronouns.

Krebs and colleagues investigate the neural correlates underlying the processing of word order variations in Austrian Sign Language (ÖGS) (Krebs et al., 2018; Krebs, Malaia, et al., 2019). Here, participants are presented with signed sentences in SOV and OSV orders, containing two arguments overtly linked to R-loci in the signing space followed by either an agreement verb or a plain verb in combination with an overt agreement marker, which is used in ÖGS to express agreement overtly with plain verbs. Krebs et al. (2018) report lower acceptability ratings; longer reaction times and different ERPs to OSV compared to SOV structures, that are interpreted as reflecting increased processing of the OSV structure. These data, therefore, provide the first empirical evidence for the presence of a subject preference in ÖGS, thereby demonstrating its modality independence. As a follow-up, Krebs et al. (2019) examine whether the observed subject preference can be modulated or eliminated in the context of sign language specific topic constructions. Behavioral data do not show any difference between conditions and word orders. However, differences in the ERPs to OSV compared to SOV structures are again taken as indexing increased processing costs for OSV structures. Together, these studies provide neurophysiological evidence for the presence of a subject preference even in sign language specific constructions. 
Overall, these studies present data on the first mention effect and the subject preference as modality-independent mechanisms in language comprehension. However, it remains unclear whether the first mention effect and the subject preference are distinct processes or whether they describe the same or at least similar phenomena for sign languages. In the syntactic analysis of sign languages, it is still an ongoing debate how to define and use grammatical categories such as subject and object. Some sign languages are argued to determine their word order based on grammatical relations in that subjects typically precede objects (Li \& Thompson, 1976; Lillo-Martin, 1986; Meir, 2002). In contrast, other sign languages are reported to base their word order not on grammatical relations but on discoursefunctional relations (for a review, see Leeson \& Saeed (2012)). Consequently, the existence of grammatical subjects and objects are controversially discussed for these languages, with some people arguing that the categories subject and object are not relevant (or even misleading) for the linguistic description of sign languages (Bouchard \& Dubuisson, 1995; Li \& Thompson, 1976; Lillo-Martin, 1986). Despite this discussion, grammatical subjects precede grammatical objects in basic word order of most sign languages, thus complicating the differentiation between first mention effects and subject preference. Therefore, we will use the term first mention in our study as this term does not depend on specific syntactic assumptions and provides a theory neutral approach. Distinguishing between first mention and subject preference is not in the scope of this paper and is left for future research.

\subsubsection{The current study}

As presented above, previous research suggests the first mention effect to be a universal and modality-independent mechanism in language comprehension. Importantly, the effect is reported for unambiguous contexts where referents have been localized in the signing space. Therefore, the question arises whether a similar first mention effect can be observed for 
sign languages when referents have not been overtly localized in the signing space. To examine the first mention effect in DGS, we investigate whether pronominal INDEX signs directed to either the ipsilateral or the contralateral side of the horizontal plane evoke different neurophysiological effects. In contrast to (Krebs et al., 2018; Krebs, Malaia, et al., 2019), referents in the present study will not be overtly localized in space. We are re-assessing the data from Wienholz et al. (2018) by timelocking the analysis to an earlier position in the stimulus material as explained below.

While recording their EEG, participants are presented with short strings of discourse consisting of two sentences (same material as used by Wienholz et al. (2018)). In the first sentence, two referents are introduced (e.g., PRIEST and GRANDMA as in subsequent examples (1) and (2)), but not assigned overtly to an R-Locus by any of the manual or nonmanual strategies mentioned above. ${ }^{6}$ The second sentence always starts with a pronominal pointing sign, i.e., the INDEX sign, followed by two additional signs. ${ }^{7}$ As already mentioned above, participants appear to follow a default pattern in interpreting the antecedents of the pronouns in the sentences (Wienholz, Nuhbalaoglu, Mani, et al., 2018). Based on this default pattern, the referent introduced first is implicitly assigned to the ipsilateral side whereas the second referent is established at the contralateral side. Thus, in the ipsilateral condition, the INDEX

\footnotetext{
${ }^{6}$ Notational conventions: signs are glossed in small caps. In the presented example sentences, index is always used as a pronoun referring back to one of the two referents introduced in the first sentence. Subscript ' 3 ' refers to the region in the horizontal plane used for third party referents whereas ' $a$ ' (right/ipsilateral) and ' $b$ ' (left/contralateral) denote the lateral sides in the horizontal plane. Glosses in bold indicate the pronoun and its (potential) antecedent. Note that DGS is assumed to be an SOV language (Happ \& Vorköper, 2006).

${ }^{7}$ The additional signs are not relevant for the purpose of this paper since they did not have an influence on the effects measured on the preceding index sign. See Wienholz et al. (2018) for a more detailed description of these signs.
} 
sign is directed to the ipsilateral/right (INDEX3a) side in the signing space expecting the continuation to refer to the first mentioned referent (Example 1). Sentences in the contralateral condition contained an INDEX sign directed to the contralateral/left side in the signing space anticipating a continuation referring to the second mentioned referent. In addition, all utterances contain a sentence-final predicate in first sentences, that does not require an additional agreement marker.

(1) Ipsilateral condition:

a. DGS: PRIEST GRANDMA MEET. INDEX 3a HAVE BEARD.

German: Ein Priester trifft eine Oma. Er hat einen Bart.

'A priest meets a grandma. He has a beard.'

b. DGS: GRANDMA PRIEST MEET. INDEX 3a HAVE BEARD.

German: Eine Oma trifft einen Priester. Sie hat einen Bart.

'A grandma meets a priest. She has a beard.'

(2) Contralateral condition:

a. DGS: PRIEST GRANDMA MEET. INDEX 3 b HAVE BEARD.

German: Ein Priester trifft eine Oma. Sie hat einen Bart.

'A priest meets a grandma. She has a beard.'

b. DGS: GRANDMA PRIEST MEET. INDEX 3 b HAVE BEARD.

German: Eine Oma trifft einen Priester. Er hat einen Bart.

'A grandma meets a priest. He has a beard.' 
We expect the preference for either interpretation to be reflected in the modulation of the N400 ERP component. This negative-going component peaks between 300 and $500 \mathrm{~ms}$ following stimulus onset over central and parietal regions across either the left or right hemisphere (for a review, see Kutas \& Federmeier (2000, 2011)). Traditionally, the N400 is interpreted as reflecting processes underlying meaning processing and is modulated by lexical-semantic factors (Lau et al., 2008). However, this component has also been shown to reflect a reanalysis of grammatical functions, such as a subject-object reanalysis (Haupt et al., 2008). The same ERP component has been observed in sign language processing as well. In ASL, sentences containing or ending with a semantic violation evoke an N400 effect, just as the same violations for spoken languages do (Capek et al., 2009; Kutas et al., 1987). Similarly, semantic violations show a centrally distributed N400 in DGS (Hänel-Faulhaber et al., 2014; Hosemann et al., 2013). Hosemann et al. (2013) observe the N400 in the transition phase, i.e., the phase between two signs, and therefore before the onset of the critical sign. This suggests that detection of semantic anomalies is already triggered by information contained in the transition phase. This finding is further supported by Krebs et al. (2018) and Krebs, Wilbur, et al. (2019) who show that the processing of object-initial structures in ÖGS evoke a negativity effect before the start of the disambiguating element even in sign language specific constructions.

Thus, regarding our study, if signers have a preference for one of the referents to continue with in subsequent discourse, we expect increased deflections for conditions violating this preference. In keeping with the first mention effect, we predict an increased negativity for the contralateral condition compared to the ipsilateral condition reflecting increased processing costs when referring to the second mentioned referent. However, if we observe increased brain activity in the ipsilateral condition with higher processing costs for first mentioned referents, signers do not show a preference for the first but rather for the 
second mentioned referent. Finally, ERPs might not differ across conditions indicating that there is no preference for either referent.

\subsection{Methods}

\subsubsection{Participants}

In this study, 21 (12 female, 9 male) deaf native signers of DGS (age range: 20-51 years; mean: 33 years) from different regions of Germany participated as paid volunteers. Eighteen were congenitally deaf and three became deaf between the age of 16 and 36 months. All participants had deaf parents or DGS input before the age of three years, were all righthanded (checked in a handedness test), had at least high school education level and normal or corrected-to-normal vision. During data analysis, one participant was excluded due to excessive eye movement artifacts. The Ethics Committee of the Institute of Psychology at the University of Goettingen provided ethics approval for the experiment. All participants received information about the EEG technique and the procedure in DGS and German and gave written signed consent prior to the experiment.

\subsubsection{Material}

In total, 240 stimulus sentences were presented comprising 160 experimental stimuli with 80 stimulus sentence sets for each condition (a full list of the stimulus material is provided in Appendix B) and an additional set of 80 filler sentences. Experimental conditions (see example 1 and 2 above) differ with respect to the direction of the INDEX sign used in the sentences. Thus, the ipsilateral condition contains an INDEX sign directed to the right, i.e., ipsilateral, side whereas the contralateral condition presents an INDEX sign directed to the left, i.e., contralateral, side on the horizontal plane. Furthermore, within each condition, the first 
mentioned referent was varied so that each of the two referents occurred once as the first and once as the second mentioned referent across participants.

A stimulus sentence set is composed of two naturally signed sentences containing three signs each. The sentences show no causal relation. In the first sentence, two referents were introduced without either manual or nonmanual localization, followed by a transitive non-localizing verb adhering to the neutral SOV word order in DGS. Referent combinations were used maximally twice while each referent occurred maximally ten times. Only animate referents were used so that the referent order could be reversed without changing the syntactic and semantic well-formedness of the sentences so that either referent could take the position of the agent/subject. Due to potential increased processing costs involved in the processing of compound signs, i.e., signs consisting of at least two signs to form one meaning, and proper names, i.e., fingerspelled or conventional name signs, these were never used as referents. To exclude localization with the verb, only non-localizing transitive verbs were chosen.

Therefore, agreement verbs requiring spatial anchoring of referents and some non-localizing, i.e., plain, verbs obligatorily requiring an agreement marker directed toward the region in space where the referent was introduced were excluded (Rathmann, 2003). Moreover, verbs needed to allow for the reversed order of referents without changing the semantics and the appropriateness of the sentences. Noun pair-verb combinations occurred only once while verbs itself were repeated maximally four times.

Each second sentence started with an INDEX sign followed by two additional signs. The INDEX varied in the direction towards a corresponding region in the horizontal plane. In all sentences, the INDEX took the function of a pronoun referring either to the ipsilateral or the contralateral side of the signing space. Effects are measured at the INDEX so that participants are yet to see the rest of the second sentence at this particular time. Thus, the material 
following the INDEX ought not to influence the INDEX's processing and is not of interest for the purpose of the current study. ${ }^{8}$

Given dialectal variations of DGS (Eichmann \& Rosenstock, 2014; Langer, 2012; Macht \& Steinbach, 2019), we verified whether participants understood the referent signs as intended. The comprehension task was conducted prior to the experiment and ought not to impact the ERP data since we did not analyze ERP waveforms to these signs. All referent signs were presented as isolated single signs with their corresponding meaning (German word) as a subtitle. Participants had to indicate with a button press whether they understood the sign with the presented meaning even if they would not use the sign themselves. Familiarity of each participant with all items and familiarity of each item to the participants was analyzed. Participants on average knew $97 \%$ of the items (range: $74-100 \%$ ) and items were familiar to participants on average $97 \%$ as well (range: $76-100 \%$ ). Thus, no participant and no item were excluded from the analysis based on the comprehension task.

The sentence sets were signed by two male right-handed deaf native signers and recorded using a Sony HDR-CX550VE camcorder (25 frames per second) from frontal view. Verbs were signed in the neutral space in front of the signer to exclude overt spatial localization. Moreover, we controlled for the usage of nonmanuals during video recordings so that the signers avoided eye gaze shifts, body movements, head nods and eyebrow raise as their role in the processing of sign languages is still under-investigated. However, the signers

\footnotetext{
${ }^{8}$ The material in the second sentence following the index is constructed the way that the second sentence unambiguously refers back to one of the referents introduced in the first sentence. Thus, the example sentences (1b) and (2a) result in a semantic mismatch at the final sign of the second sentence. These data are reported in Wienholz et al. (2018).
} 
used lexical nonmanuals and mouthing since both markers help to disambiguate between signs in DGS (Ebbinghaus \& Heßmann, 2001). There was a natural prosodic break between the two sentences. Videos were then edited using Adobe Premiere Pro CS6 such that the signer remained motionless for $2000 \mathrm{~ms}$ at the beginning and $1500 \mathrm{~ms}$ at the end of each video and that videos had a size of $1280 \times 720$ pixels. Each sentence set was recorded separately so that no further splicing within the video was necessary. Videos had a duration of $8550 \mathrm{~ms}$ on average $(7000-9320 \mathrm{~ms})$ with no significant difference of duration across conditions (Ipsilateral condition: $8539 \mathrm{~ms}$; Contralateral condition: $8561 \mathrm{~ms}$ ).

Additionally, a set of six practice items, four of them similar to the experimental and two similar to the filler sentences, was constructed and used to familiarize participants with the procedure. All practice items were constructed and recorded in the same way as sentences in the main experiment. Sign combinations in the practice experimental stimuli were not used in the main experiment.

\subsubsection{Procedure}

The study was conducted at the Experimental Sign Language Lab at the University of Goettingen. To guarantee a DGS language environment, a native signer of DGS supported the experimenters during testing. The experiment was preceded by a comprehension task and followed by a posttest. ${ }^{9}$ Participants were seated in a top-lit cabin on a chair at a distance of approximately $1 \mathrm{~m}$ from a 24 -inch screen. Stimuli were presented using the experimental software Presentation 18.1 (Neurobehavioral Systems, Inc.). Participants watched a DGS instruction video explaining that they would see multiple videos with DGS sentences and

\footnotetext{
${ }^{9}$ For a description and the results of the posttest, see Wienholz et al. (2018).
} 
would be asked after each video to judge the sentences. Additionally, they were instructed to reduce body movements and, if possible, to blink after the presentation of each video. The practice items were presented to familiarize participants with the trial structure (Figure 2.1) and could be repeated as often as required by participants. Each trial started with a black blank screen for $500 \mathrm{~ms}$ followed by a video presenting one sentence set in the center of the screen in the natural speed of signing. Following each video, a question mark appeared signaling participants to provide their judgement of the presented sentences. Participants were instructed to judge the sentences based on their "gut feeling". ${ }^{10}$ They had to indicate, via button press on a Microsoft Xbox 360 Wired Controller, whether the sentence was good, i.e., pressing the green 'thumbs up' button, or bad, i.e., pressing the red 'thumbs down' button. There was no time limit given for the response time.

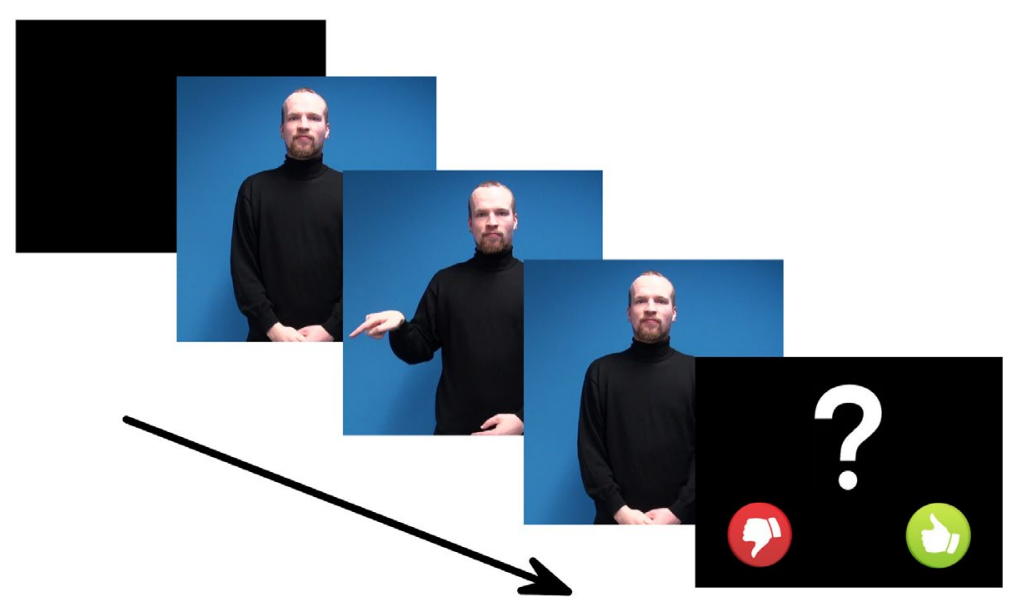

Figure 2.1 Structure of a single trial

Stimuli sentence sets were pseudo-randomized into 10 blocks of 24 sets each in four different lists. Each block contained 16 experimental stimuli, i.e., eight from each condition,

\footnotetext{
${ }^{10}$ Analysis and discussion of the behavioral data are presented in Wienholz et al. (2018).
} 
and eight filler sentences. Sentences within a block were all from different sentence sets and their order was randomized on-line by the experimental software.

\subsubsection{EEG-recording}

The EEG data were recorded using 64 active $\mathrm{Ag} / \mathrm{AgCl}$ electrodes placed according to the international 10-20 system and were amplified using an Active Two AD-Box (BioSemi B.V., Amsterdam, Netherlands) with a sampling rate of $2048 \mathrm{~Hz}$. The electrooculogram (EOG) was monitored using two external electrodes at the temple on each side (horizontal EOG) and one external electrode below the right eye in combination with electrode Fp2 on the cap (vertical EOG). Impedances were kept below $20 \mathrm{k} \Omega$ and data were re-referenced offline to linked mastoids. EEG data were analyzed using the ERPLAB toolbox plugin (LopezCalderon \& Luck, 2014) for MATLAB (The MathWorks, Inc.). First, the EEG signal was filtered using a $0.1-30 \mathrm{~Hz}$ band-pass filter and was down-sampled to $250 \mathrm{~Hz}$. Then, ERP averages were calculated for each participant for each electrode and condition. Two independent coders manually identified the following trigger positions for each video using the ELAN software (Wittenburg et al., 2006). The coders mainly agreed on trigger positions. Cases of disagreement were discussed by the two coders until consensus was reached. No further inter-rater reliability was performed.

1. Sign offset: Last frame of the final hold of the sign preceding the critical sign before the hand gets relaxed again and before the movement of the transition phase starts. This trigger position was used for baseline correction.

2. Direction: The frame when the direction of the INDEX becomes clear due to the movement of the dominant hand towards one or the other side of the signing space. 
The sign might still point to one area, but the movement clearly indicates this sign's direction to another area.

ERPs were timelocked to the trigger 'direction' and baseline corrected 200 ms prior to sign offset triggers of the preceding sign as we aim to target the effects starting already at the introduction of the critical sign. We did not baseline correct to the time window immediately prior to the trigger 'direction', since processing of the sign already begins in the transition phase preceding the sign (Hosemann et al., 2013). Trials with artifacts, e.g., blinks or muscle movement, exceeding the artifact rejection threshold of $100 \mu \mathrm{V}$ were rejected before averaging. Based on previous sign language processing research reporting N400 effects and on results from t-tests, further analysis will focus on differences within the $400-500 \mathrm{~ms}$ time window (Hosemann et al., 2013; Krebs et al., 2018).

\subsection{Results}

For the analysis, lateral electrodes were combined according to the regions and hemisphere as follows: fronto-central (F3-FT7 \& F4-FT8), centro-temporal (C3-/TP7 \& C4TP8) and parietal-occipital (P3-PO7 \& P4-PO8) (see Appendix B for a schematic illustration of the regions). In the previously identified $400-500 \mathrm{~ms}$ time window, visual data inspection suggested a negativity for fronto-central and parietal-occipital regions on the right hemisphere (Figure 2.2). A $2 \times 3 \times 2$ ANOVA with the factors hemisphere (left, right), region (frontocentral, centro-temproal, parietal-occipital) and condition (ipsilateral, contralateral) revealed a significant main effect for region $(F(2,38)=9.178 ; p=.004)$ and a near significant interaction of hemisphere* condition $(F(1,19)=4.326 ; p=.051)$. The Greenhouse-Geisser correction was applied as required (Greenhouse \& Geisser, 1959). 


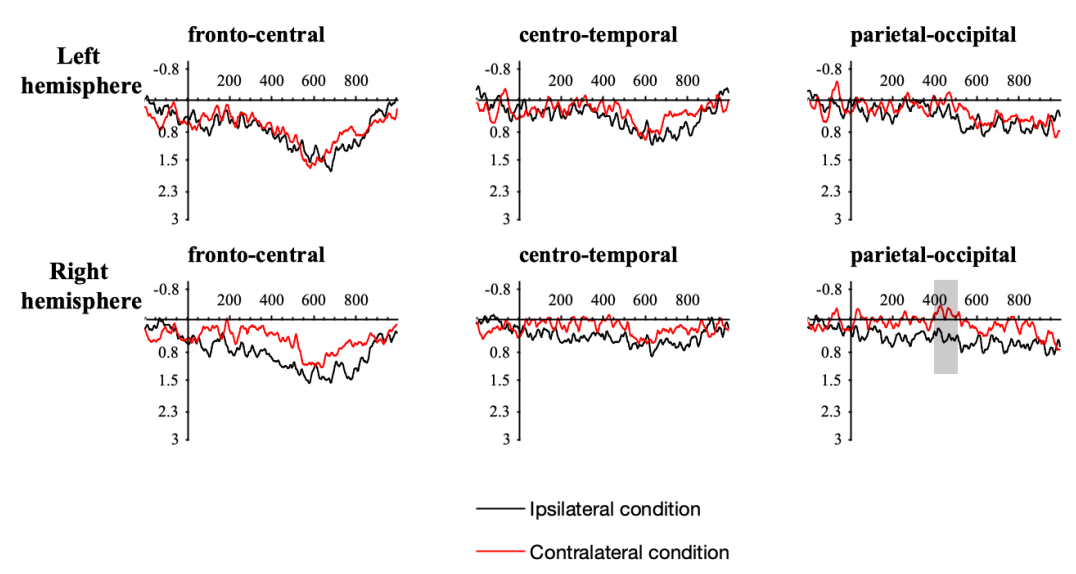

Figure 2.2 Grand average ERPs for ipsilateral (black) and contralateral (red) conditions timelocked to the trigger 'direction' for fronto-central, centro-parietal and parietal-occipital regions across left $(\mathrm{LH})$ and right hemisphere $(\mathrm{RH})$. Negativity is plotted upwards.

Splitting for hemisphere, a 3x2 ANOVA for the left and right hemisphere separately with the factors region (fronto-central, centro-temporal, parietal-occipital) and condition (ipsilateral, contralateral) revealed a significant main effect of region $(F(2,38)=6.924 ; p=$ .009) for the left hemisphere. For the right hemisphere, the analysis showed a significant main effect of region $(F(2,38)=7.822 ; p=.004)$ and a near-significant main effect of condition $(F(1,19)=3.717 ; p=.069)$. Paired samples t-tests comparing ipsilateral and contralateral conditions only revealed a significant difference on the right hemisphere for the parietaloccipital region $(t(19)=2.236 ; p=.038)$ with the contralateral condition $(M=-0.436)$ being more negative relative to the ipsilateral condition $(M=0.783)$, providing weak evidence for the suggestion of increased processing costs when the contralateral INDEX is presented, i.e., when the location consistent with the second mentioned referent is presented as the continuation of the discourse. No other comparisons reached significance.

\subsection{Discussion}


This study examined the presence of the first mention effect in DGS by investigating neurophysiological effects evoked by pronominal pointing signs. We presented participants with two-sentence discourses containing two referents. Both referents were introduced without any overt localization cue in the first sentence followed by an ipsilateral or contralateral pronominal pointing sign in the second sentence. Based on the default pattern for the assignment of referents to an R-locus in the signing space and depending on its direction (Wienholz, Nuhbalaoglu, Mani, et al., 2018), the pronoun refers either to the first or second referent. Thus, ipsilateral pronominal pointing signs refer to the first referent and contralateral pointing signs refer to the second mentioned referent. The results offer evidence of increased negative deflections timelocked to the trigger position 'direction' of the pronominal pointing sign in the 400-500 ms time window for the contralateral condition compared to the ipsilateral condition in the parietal-occipital region on the right hemisphere. In particular, we found increased processing costs associated with processing of the contralateral pointing sign, the location associated by default with the second mentioned referent. This could be interpreted as evidence for a first mention effect for DGS.

The interpretation of the observed N400 as a first mention effect in DGS is based on previous findings showing that DGS users follow a default pattern in assigning referents to Rloci covertly (Wienholz, Nuhbalaoglu, Mani, et al., 2018). According to this default Steinbach \& Onea (2016), the referent introduced first in the discourse is assigned to the ipsilateral side, while the referent mentioned second is assigned to the contralateral side of the signing space. Extending this to the stimuli presented in our study, the INDEX sign referring to the ipsilateral side picks up the first mentioned referent, while the INDEX sign referring to the contralateral side picks up the second mentioned referent. The N400 triggered by the contralateral INDEX in the current study suggests that participants expect the second sentence to continue with the 
first mentioned referent, i.e., the ipsilateral INDEX sign, and incur additional processing costs following violation of this expectation due to presentation of the contralateral INDEX sign.

As outlined above, Krebs et al. (2018) argue for a subject preference in ÖGS, such that discourse continuations that pick up the object of a previous sentence cause additional processing costs relative to continuations that pick up the subject of this sentence. There are however, three crucial differences between the current study and Krebs et al. (2018). First, we note that referents were not overtly localized in the current study. Furthermore, we investigated the influence of ambiguous pronoun usage across sentences, while Krebs et al. (2018) examined word order variations. Finally, we ensured that we only used non-localizing verbs as these do not use localizations in the space to express referential relations in contrast to agreement verbs that do rely on spatial information. Despite these differences, the results of both studies hint towards a first mention effect in DGS. In particular, we note that in DGS, basic word order is described as SOV (Happ \& Vorköper, 2006). Our stimulus material, in keeping with this basic word order, similarly had the first referent taking the position of the grammatical subject and the second referent as grammatical object. To this extent, the effect in our study could equally be interpreted as an effect of subject preference, such that the pronoun in the ipsilateral condition would refer back to the grammatical subject while the contralateral pronoun would pick up the grammatical object. Therefore, the effect would reflect increased processing costs when the discourse continues with the object, indicating a subject preference in discourse continuations. This interpretation would be in line with observations reported by Krebs et al. (2018) for ÖGS and comparable with the forced choice data from Frederiksen \& Mayberry (2017) reporting a subject preference for ASL. At present, we are unable to tease apart whether subject preference and first mention effects are two distinct processes or just different ways of referring to the same phenomenon especially in 
languages with SOV word order. Further research in sign languages with variable word order could be used to tell apart these two concepts.

A similar difference triggered by the processing of ipsilateral or contralateral INDEX signs was observed by Wienholz et al. (2018) using the same stimulus material. Comparing (congruent) trials based on the direction of the INDEX sign revealed increased processing cost for the contralateral direction between 500-600 ms. One difference between the results in this study and the previous work is that the effect was observed only across the left hemisphere in Wienholz et al. (2018). Given that the current study measured ERPs timelocked to when the direction of the sentence-initial INDEX sign was clearly predictable, compared to ERPs timelocked to the beginning of the sentence-final sign in Wienholz et al. (2018), we suggest that the left hemisphere specific effect in Wienholz et al. (2018) may have been triggered by processing of additional contextual information prior to sign processing.

Indeed, while both hemispheres of the brain are shown to be involved in language processing (for a review, see Burgess \& Chiarello (1996)), the right hemisphere plays an especially important role in discourse processing. Initial evidence in keeping with this suggestion comes from studies examining patients with right hemisphere brain damage, who display difficulties in discourse level processing. Difficulties range from impairment in topic maintenance and metaphor comprehension, while nevertheless showing full understanding of single sentences and single words (Burgess \& Chiarello, 1996; St George et al., 1999). Neurophysiological studies similarly provide evidence for right hemispheric involvement (in addition to left hemisphere activity) in processing semantic and pragmatic information, suggesting that this hemisphere is associated with comparing new information to previously obtained context information and maintaining a coherent discourse (Federmeier \& Kutas, 1999; St George et al., 1999). The few case studies on sign languages that are available 
suggest a similar involvement of the right hemisphere in discourse processing (Hickok et al., 1999). Data obtained from signers with right hemispheric brain damage show inconsistent use of R-loci, i.e., switching between horizontal and midsaggital plane, difficulties in topic maintenance and role shift while production and comprehension of signs and sentences continues to be unimpaired (Emmorey, 2002; Hickok et al., 1999; Loew et al., 1997). Taken together, the N400 effect observed here and its right hemispheric distribution across the scalp might be attributed to the discourse processing focus of the current study. Therefore, the neurophysiological data might indicate right hemispheric involvement in semantic-pragmatic discourse processing in sign languages.

One final caveat that needs to be addressed here is the possibility that the N400 effect reported here may be triggered purely by processing of the spatial location of the referential expression. In other words, it is possible that the observed ERP differences are due to differential processing of the ipsilateral or contralateral area in the signing space rather than due to the pronoun disambiguation as suggested here. We suggest that this explanation is unlikely, given that the two distinct areas in the space should evoke an effect of visuospatial processing, which typically occur quite early, usually within the first $200 \mathrm{~ms}$ post-stimulus (Luck, 2005). The absence of temporal overlap between the visual components expected in such a case and our findings suggests that the reported effect is unlikely to be due to early differences in visuospatial processing of right and left areas of the signing space. Equally, we note that a further alternative interpretation of the observed effect is an index of mismatch between the expected continuation and the presented stimulus expectation, specifically targeting the visual mismatch negativity (MMN, e.g., Kimura et al. (2011)). However, we note that the visual MMN peaks earlier, between 250-400 ms post-stimulus, and consequently does not offer a reasonable interpretation of our observed effect, similar to other earlier visual components. To rule out perceptual effects (Neville et al., 1997), the experiment needs to be 
tested with non-signers watching the sign model pointing to either side of the signing space. If this group shows similar effects as the DGS signers, physical differences of the pointing signs rather than their linguistic information would yield the observed effect. In contrast, if they perform differently, this would suggest that signers associate the spatial directions with language-specific discourse-pragmatic function. We leave this for future research.

\subsection{Conclusion}

This study provides further evidence that the first mention effect is modalityindependent and applies to spoken and sign languages to the same extent. In particular, we show that participants incurred increased processing costs when the discourse continuations referred to the second mentioned referent in the discourse relative to the first mentioned referent. We note that while the phenomena of first mention effect and subject preference can be distinguished in some spoken languages, the extent to which the two effects can be separated within a specific sign language or whether the occurrence of the effect can be generalized across sign languages remains unclear. Since sign languages do not show overt case marking (Sandler \& Lillo-Martin, 2006), they might behave similar to their spoken language equivalents such as English. We rather need to test passive constructions where the object can be mentioned first or other constructions where objects are fronted to be able to disentangle the two phenomena. However, there is more work needed to look at the basic word order and its variations in DGS as well as discussing the terminology of grammatical subject and object in sign languages in general. Additionally, further studies should focus on examining effects evoked in other sign language specific structures such as topic constructions to allow for generalization of these findings. 



\title{
3 The influence of overt localization on the processing of referential expressions in German Sign Language
}

\begin{abstract}
Processing of referential expressions is not only influenced by its form, but also by the accessibility of their antecedents. The present study examined whether overt manual localization increases the prominence and hence the accessibility of a discourse referent and how this interacts with the grammatical role of the referent in German Sign Language. Using eye tracking and a modified version of the Visual World Paradigm, participants were presented with two pictures representing the discourse referents contained in the stimulus sentence presented in a video. Each video included short discourses that introduced two discourse referents with varying their overt localization in a first sentence while a subsequent second sentence started with one of the referents, i.e., either the previous subject or object as a bare noun. Analyzing proportions of target looking using mixed-effects models revealed increased looks to the target referent for conditions containing overt localization of both referents or only localizing the subject. This suggests that overtly localizing a referent indeed enhances its accessibility, but only if the referent occurs in the subject position. Moreover, localization seems to accentuate a referent in a similar way as prosodic focus in spoken languages. Thus, the combined factors of localization and subject preference lead to facilitatory processing of referential expressions co-referential with the focused referent.
\end{abstract}

Keywords: German Sign Language, eye tracking, referential expressions, accessibility 


\subsection{Introduction}

Sign languages exploit the horizontal plane of the signing space to assign discourse referents to referential locations (henceforth, R-locus; Liddell, 1990; Lillo-Martin \& Klima, 1990). In the subsequent discourse, these locations are used to establish a relation between a referent and a referential expression. One way to associate a referent with a specific R-locus is to point with the index finger ${ }^{11}$ (INDEX sign) towards that location. Often, pointing signs take multiple functions at the same time such as a localizing and a determiner function (Neidle \& Nash, 2012). The question arises whether the overt assignment of a referent affects its accessibility and/or increases its prominence leading to facilitative processing of a subsequent co-referential expression. In spoken languages, the comprehension of a referential expression is affected by the form of that expression, but is also influenced by the accessibility or prominence of its antecedent (Almor \& Eimas, 2008; Ariel, 1990; Gundel et al., 1993). In general, referential expressions are assumed to refer to the most accessible referent, which is the most activated, in the discourse context (Gordon et al., 1993). The accessibility of a discourse referent can be modified by various factors, that differ in their strength of influence (for an overview, see Arnold, 1998).

\subsubsection{Prominence and its effects on processing in spoken languages}

A vast literature of psycholinguistic research provides evidence for an effect of grammatical role during the comprehension of a referential expression such that referents occurring in subject position are more salient and thus more likely to be interpreted as

${ }^{11}$ Pointing signs fulfill various grammatical functions such as determiner, adverbials or personal pronouns (Bahan et al., 1995; Cormier et al., 2013; MacLaughlin, 1997). 
antecedent of a referential expression (Gernsbacher, 1989; Gernsbacher et al., 1989; among others). Often, this effect is referred to as subject preference. When producing and comprehending referential expressions, participants showed a preference to interpret subjects as antecedents suggesting that subjects are more prominent in the working memory (Crawley \& Stevenson, 1990). Results of a probe recognition task conducted by Gernsbacher (1989) indicate that referential expression of various forms trigger the enhancement of antecedents accessibility while suppressing non-antecedents. Kaiser (2011) presents supporting data for subject preference in the interpretation of ambiguous pronouns. This study shows increased looks to a subject referent in an eye tracking setting and higher accuracy in a behavioral task detecting mismatches of the auditory and the visual stimulus material. Thus, participants' interpretation of referential expressions seems to be strongly influenced by subjecthood.

Another factor concerns the status of an information suggesting that being given in previous discourse increases the salience of that information while discourse new information are less salient. This factor is connected to the notion of topicality in the present discourse, i.e., what a sentence or a proposition is about (Givón, 1976; Kaiser, 2006). The Giveness Hierarchy assumes that referents are cognitively more prominent when they are introduced as topics (Gundel et al., 1993). This claim is supported by Cowles et al. (2007), who examined the effects of prominence marking in a cross-modal naming task, i.e., presenting stimulus sentences auditorily followed by a visual item that had to be named. Topicalized antecedents showed shorter naming times suggesting enhanced cognitive prominence with similar effects evoked by focused antecedents. Thus, topic and focus seem to serve a similar function of enhancing the prominence of their referents within the mental representation similarly for first and second mentioned referents (Arnold, 1998; Cowles et al., 2007). 
Just as a topicalized element, a focused referent is more likely to be the antecedent of a referential expression so that this referent is more salient as its accessibility in the mental representation is increased (Cutler \& Fodor, 1979; Gundel et al., 1993). Focus can be marked syntactically using a cleft-sentence construction or prosodically by using specific intonation, i.e. accentuation (for an overview, see Arnold, 1998). A series of experiments by Birch et al. (2000) showed that syntactically focusing a referent increases its salience and leads to a stronger representation within working memory. During a story continuation task, focused constituents were more likely to be referred to in subsequent discourse and in a probe recognition task, a focus effect occurred when the probe was presented with a delay while probes immediately following the presented discourse showed a subject preference (Birch et al., 2000). Eye tracking data provided by Foraker \& McElree (2007) suggest the strengthening of an antecedent's mental representation as well which leads to increased likelihood of being retrieved in subsequent discourse. Moreover, the authors indicate that the interpretation of ambiguous pronouns seemed to be driven by properties of possible antecedents such as grammatical role or their position in the sentence. This is compatible to observations by Arnold (1998) showing as well that focused elements are more often referred to in a cleft construction and that in the absence of a cleft-construction the subject is the most likely referent. In contrast, Almor (1999) showed using a self-paced reading task that definite noun phrase anaphora are read faster when they refer to a focused antecedent irrespective of its grammatical role. Another study by Almor \& Eimas (2008) showed an overall facilitation effect for noun phrase anaphora co-referential with a focused referent in a lexical decision task while repeating the focused noun phrase as a referential expression lead interference effect in a memory recall task reflected in poorer accuracy. The syntactic focus effect can be strengthened by adding contrastive intonation suggesting that both strategies conjoined add to the focus effect (Cowles et al., 2007).Using a probe recognition task, Gernsbacher \& Jescheniak (1995) investigated intonation as a cataphoric device and how this influences the 
accessibility of the focused referent. Results showed faster response times when the focused referent was probed and slower response to the same probe if another referent was prosodically marked. Therefore, they argue that intonation increases the accessibility of a referent by enhancing its activation and suppressing others. In the end of their literature review on the influence of prosody on language comprehension, Cutler et al. (1997) conclude that prosodic information does not provide discourse-structural information itself but rather it marks a constituent in the discourse which will be referred to later leading to easier processing of this referent.

\subsubsection{Prominence and its effects on processing in sign languages}

There are only a few studies looking at the factors affecting referent accessibility in sign languages. Nevertheless, grammatical role seems to affect referents' accessibility in spoken and sign languages suggesting that the subject or first mention preference is a modality-independent factor. Data from event-related potential (ERP) studies and a forced choice task provide supporting evidence for the presence of such an effect in different sign languages (Frederiksen \& Mayberry, 2017; Krebs et al., 2018; Wienholz et al., submitted).

In sign languages, a change in word order seems to be a widely used strategy to modify the information structure of an utterance ${ }^{12}$. In American Sign Language (ASL), the sentence-initial position seems to be highly prominent and hence used to mark an element as the topic (Fischer, 1975; Liddell, 1980). One possibility to get an element into this position includes the movement of a constituent from its original position within a sentence in neutral word order to the front position in the left periphery (Neidle, 2002). However, the same

\footnotetext{
12 See Wilbur (2012) for an overview of topic and focus constructions and marker in sign languages.
} 
position might be occupied by contrastively focused elements suggesting it is used generally to shift the attention to that specific element (Neidle, 2002; Wilbur, 1997). In contrast, doubling an item in or moving it to the sentence-final position is suggested to increase its prominence as well (Lillo-Martin \& de Quadros, 2008; Wilbur, 1997). Wilbur (1999) suggests that prosodic stress, i.e., greater movement or longer duration of a sign, serves similar functions across modalities only differing in the form it is expressed. Therefore, prosodically marking a constituent at the end of a phrase might lead to focusing that element and thus increases its prominence (Wilbur, 1997, 1999). Moreover, manual signs such as only or even serve as focus particles, but often they need to be accompanied by a nonmanual marker to fulfill the focus function suggesting that nonmanual markers are an obligatory part of topic and focus constructions (Herrmann, 2014; Wilbur, 2012). For ASL, the combination of eye brow raise and backward tilt of the head is described as marking topics ${ }^{13}$ (Aarons, 1994). Moreover, forward and backward body leans reinforce the most prominent element in an intonation phrase in ASL, usually the element in sentence-final position (Wilbur \& Patschke, 1998). For sign language of the Netherlands, van der Kooij et al. (2006) describe a similar function of body leans as increasing the prominence of a focused item. They analyze forward body leans as a prosodic information focus marker at least for grammatical subjects. Looking at contrastive focus, the authors observe a left-right spatial contrast of body leans (van der Kooij et al., 2006). Nevertheless, there is no clear cut between the different nonmanual markers and their function since each of them seems to serve a number of functions. Often, they occur in combination with other nonmanual or with a manual component in specific contexts. To the best of the authors knowledge, no study so far investigated whether one of the mentioned strategies or markers is indeed increasing the prominence of an item and thus enhances its accessibility. However, each of the constructions

\footnotetext{
${ }^{13}$ See Aarons (1994) and Neidle (2002) for a syntactic analysis of nonmanual markers.
} 
and markers described above need to be investigated and described in more detail to determine their influence on processing mechanisms.

\subsubsection{The current study}

The current study examines the influence of overt localization on the processing of discourse referents in German Sign Language (DGS). Specifically, we ask whether overt localization of a discourse referent facilitates its processing when this referent is picked up later. Moreover, we investigate whether effects differ with respect to the grammatical function of the localized referent, i.e., whether the referent is a grammatical subject or a grammatical object.

We presented participants with two-sentence discourses that introduced two discourse referents in the first sentence, i.e., TEACHER ${ }^{14}$ and GIRL in (1) and COOK and WOMAN in (2) (see Appendix D for video stills). Across the four conditions, the overt localization of these referents varied so that either both, only one or none of the referents were assigned overtly to an R-locus in space (see examples 1 and 2). In the subject localization condition, the subject is localized on the ipsilateral/right (INDEX $)$ side of the signer while the object is not overtly localized as in (1b) and (2b). The object localization condition presents the reversed order with only localizing the object on the contralateral/left (INDEX $)$ side of the signer while the subject is not overtly assigned to an R-locus (see (1c) and (2c)). We follow this order of

\footnotetext{
${ }^{14}$ Notational conventions: signs are glossed in small caps. In examples discussed below, INDEX is
} always used to localize the preceding discourse referent. Subscripts 'R' (right/ipsilateral) and 'L' (left/contralateral) refer to the corresponding side on the horizontal plane. Note that DGS is a predominantly SOV language. 
localization based on reports on a right-left default pattern that applies during referent assignment in DGS (Steinbach \& Onea, 2016; Wienholz, Nuhbalaoglu, Mani, et al., 2018). The INDEX sign takes the localizing function in this sentence establishing a connection between a discourse referent and a referential locus (R-locus). The analysis requires to compare the single localizing conditions, i.e., subject or object localization, with a neutral condition. However, it is not clear whether the presence or absence of overt localization determines the natural and neutral condition. Therefore, both options were included as separate conditions. Thus, either referent was assigned to an R-locus, i.e., the subject is localized on the ipsilateral/right and the object is localized on the contralateral/left side of the horizontal plane in the localization both condition (see (1a) and (2a)). In contrast, none of the referents is overtly localized in the no localization condition as in (1d) and (2d). The second sentence always started with picking up one of the referents introduced in the first sentence by repeating the respective noun. Thus, the subject noun was repeated in the subject continuation type, i.e., TEACHER in (1), while in the object continuation type, the object was picked up, i.e., WOMAN in (2). After the noun, three more signs followed which were as neutral as possible with respect to the two discourse referents. In addition to the video, two pictures were shown representing the subject and object referent, i.e., the picture of a cook and a woman were presented along with each sentence in (2).

\section{(1) Subject continuation type}

a. Localization both

TEACHER IXR GIRL IXL TALK. TEACHER DIFFERENT CITY BORN.

b. Subject localization

TEACHER IXR GIRL TALK. TEACHER DIFFERENT CITY BORN.

c. Object localization 
TEACHER GIRL IXL TALK. TEACHER DIFFERENT CITY BORN.

d. No localization

TEACHER GIRL TALK. TEACHER DIFFERENT CITY BORN.

Translation: 'A teacher talks with a girl. The teacher was born in a different city.'

(2) Object continuation type

a. Localization both

COOK IX $\mathrm{X}_{\mathrm{R}}$ WOMAN IX $\mathrm{X}_{\mathrm{L}}$ MEET. WOMAN A-LOT EAT CAN.

b. Subject localization

COOK IXR WOMAN MEET. WOMAN A-LOT EAT CAN.

c. Object localization

COOK

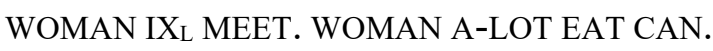

d. No localization

COOK WOMAN MEET. WOMAN A-LOT EAT CAN.

Translation: 'A cook meets a woman. The woman can eat a lot.'

To answer our research questions, we record participants' eye movements while they see video and picture stimuli presented on a computer screen. If overt localization of a referent facilitates its processing, we expect this to be reflected in the window analysis with proportionally more looks to the respective referent picture in one of the single localization conditions than in another condition. Thus, there should be more looks to the object referent in the object localization condition of the object continuation type than in another condition, i.e., more looks to the picture of the woman in $2 \mathrm{c}$ than in $2 \mathrm{a}, 2 \mathrm{~b}$ or $2 \mathrm{~d}$. Moreover, observing a 
difference in the gaze pattern comparing the two continuation types for each condition, i.e., comparing 1a with $2 \mathrm{a}$, etc., would suggest an effect of the grammatical role of the referent.

\subsection{Methods}

\subsubsection{Participants}

In total, 26 deaf native signers of DGS participated in the experiment as volunteers and were paid for their participation. However, only 23 participants (11 female, 12 male) were included in the analysis while the remaining three participants were excluded either due to too much trackloss or due to failure of calibrating the eye tracker. The included participants had a mean age of 33 years (age range: 20 - 58 years) and at least high school education level. Twenty-one participants either had deaf parents or were exposed to DGS before the age of 5 years. The other two participants were exposed to DGS at the age of 8 and 15 years, but had access to another sign language before. Moreover, both stated DGS to be their primary language for more than 20 years. All participants were informed about the procedure and gave written consent for their participation before starting the experiment and the aim/topic of the study was explained to them afterwards. The Ethics Committee of the Institute of Psychology at the University of Goettingen provided ethics approval for this study.

\subsubsection{Material}

In total, 320 sentence sets were constructed with 40 sets in each condition (a full list of the stimulus material is given in Appendix E). One sentence set was excluded before running the experiment due to an error in one of the stimulus videos so that 316 sentence sets remained for testing. Each sentence set (see section 3.1.3) consists of two sentences of the following structure. 
The first sentence contained three to five signs depending on the presented condition. There, two human discourse referents were introduced combined with a transitive nonlocalizing verb in the neutral SOV word order. As referents, we used bare nouns either in generic form, e.g., woman, as job descriptions, e.g., cook, denoting relatives, e.g., grandma, or denoting story characters, e.g., witch. However, no compound signs or proper names were used due to their more complex structure, i.e., either consisting of two connected signs to denote one meaning, using finger spelling or requiring a fixed memorized name sign, all of which might lead to increased processing load and thus might affect the looking behavior of participants. Each noun occurred maximally four times, i.e., twice as subject and object. Additionally, each noun combination only occurred once also excluding the reversed order of referents. As verbs, we used transitive spatial or reciprocal verbs signed in the neutral space in front of the signer to avoid any agreement relation between a verb and a localized discourse referent. These three signs, i.e., the two discourse referents and the verb, were kept stable across all four conditions for each sentence set. Based on the condition the sentence sets were presented in, the first sentence additionally contained either two, one or no INDEX sign.

The second sentence always contained four signs always starting with one of the noun referents from the first sentence followed by three additional signs. In the subject continuation type, the first sign was the subject while in the object continuation type, it was the object from the previous sentence. The remaining three signs of the second sentence were each only used twice while their combination was only used in one sentence set. Across the four conditions, the three signs were the same and as neutral as possible with respect to the two discourse referents. In this case, neutral should be understood such that the sentence was syntactically, but more importantly, also semantically well-formed irrespective of which referent from the first sentence was repeated. Additionally, six practice sentence sets with the same structure 
were constructed with three sets for each continuation type and minimum one set for each condition. Nouns and verbs used in the practice items were not used in the actual experiment.

The sentence sets were discussed and recorded with the help of a deaf native signer of DGS. Recordings took place in the Experimental Sign Language Lab at the University of Goettingen and were done using a Sony HDR-CX550VE camcorder. Overall, the signer was instructed to slow down the speed of signing and to keep a longer final hold of the referent sign in case of an absent INDEX sign to keep the overall length of a sentence set more or less similar across conditions. There was a natural prosodic break between the sentences with a longer final hold of the verb in the first sentence. So far, we do not know enough about the influence of nonmanuals on sign language processing. Therefore, the signer only kept mouthing and lexical nonmannuals to ensure lexical understanding, but avoided other nonmanuals, e.g., eye gaze localization of the referents, eyebrow raise or any additional head and body movement. Afterwards, videos were cut and processed using the video editing software Adobe Premiere Pro CS6 so that the signer remained motionless for 1 second at the beginning and for 1,5 second at the end of each video. Moreover, the background color of each video was changed to a grey tone that was the same in the presented picture stimuli to avoid any influence from different background colors. Videos were not further modified, had a length of 10720 - $20760 \mathrm{~ms}$ (mean duration: $14851 \mathrm{~ms}$ ) and a size of 732x550 pixels.

As picture stimuli, 26 photo-realistic images were chosen representing each of the referents. Each picture had a size of 300x400 pixels and the same grey background color as the videos. Two pictures were presented simultaneously next to each other displaying both referents introduced in the sentence set shown in the video. Pictures were chosen based on the results of a picture-naming task conducted with German native speakers in an online 
questionnaire. Using Google Forms (Google Inc.), 216 pictures ${ }^{15}$ were presented divided into four lists of 54 pictures each. Two images were presented for each noun, but in different lists, allowing to choose the most matching picture to be used in the final stimuli. Overall, 101 participants (79 female, 22 male, mean age: 25 years) named the presented image with one word. Prior to analysis, eight participants were excluded since they were no German native speakers. During evaluation, the answers to both images for each noun were compared and the picture with most correct namings was chosen for the final stimuli set. In case of no difference of the answers, the picture was chosen randomly.

\subsubsection{Procedure}

The study was conducted at the Institute of German Sign Language and Communication of the Deaf at the University of Hamburg and in the Experimental Sign Language Lab at the University of Goettingen. When participants arrived for testing, they were informed about the procedure and how the eye tracking technique works. To ensure full understanding of all information, explanations were given in DGS and in written German. Participants gave written consent and filled in a metadata form before starting the experiment. They were informed about the aim of the study only after the experiment so that they were naïve to the topic during the study.

For stimulus presentation, the sentence sets were divided into four different list so that each list only contained one sentence from each set. In total, each list contained 80 stimuli consisting of 40 sets in the object continuation and 40 in the subject continuation type. For

${ }^{15}$ Due to the preparation of a second eye tracking study in parallel (the study presented in Chapter 4), images from both studies were combined and presented in the same picture-naming task. 
each continuation type, 10 sentence sets of each of the four conditions were presented with counterbalanced picture positions, i.e., the alignment of the pictures with the side a referent was localized in the video was counterbalanced. Alignment refers to the position of the picture in relation to the occurrence of the corresponding referent in the video. Thus, in a sentence from the subject localization condition, the subject was overtly localized on the right side of the signer, which corresponds to the left side of the screen from the perceiver's perspective. Thus, if the picture of the subject referent was aligned to its localization in the video, it occurred on the left side of the screen as well. In contrast, the picture of the subject referent was presented on the right side of the screen when it was not aligned to the video. Due to counterbalancing of the picture position, each list had to be presented with the reversed picture position as well which resulted in eight different lists. Order of trials was randomized on-line by the experimental software with maximally two consecutive trials from the same condition. However, prior to testing and after compiling the randomization lists, one sentence set was excluded with all its four conditions since one of the conditions contained an error in the video. Therefore, all eight lists contained 79 stimuli in the end.

Participants were seated at a table in front of a 17-inch computer screen with a resolution of $1280 \times 1024$ pixels. To minimize head movements, they were instructed to place their head on a chinrest that was adjusted to a comfortable height for each participant individually. The eye tracker was adjusted manually to capture the participants' eye with a distance of $50-70 \mathrm{~cm}$ from the face. All stimuli were presented using a PC computer and the Eyelink Experiment Builder Software, Version 1.10.165 (SR Research Ltd.). The experiment started with an introduction video in DGS explaining the procedure followed by the practice items to familiarize participants with the eye tracking technique and the structure of the experiment. After the practice session and clarifying participants' remaining questions, the actual experiment started. In total, 79 trials were presented divided into three blocks (block 1: 
30 trials; block 2: 29 trials; block 3: 20 trials) with breaks in-between so that participants could relax their eyes and remove their head from the chinrest. Before each block, a ninepoint calibration sequence was presented and before each trial, an additional one-point drift correction, manually accepted by the experimenter, was performed.

Following the accepted drift correction, a trial continued with the presentation of the picture stimuli presented at the upper part of the screen for $3000 \mathrm{~ms}$ (Figure 3.1). After that, the video appeared at the bottom of the screen additionally to the two pictures and disappeared after the signer finished signing, i.e., when putting the hands back down. The pictures remained on the screen for additional $2000 \mathrm{~ms}$. Then the next trial automatically started. Videos were presented at the bottom of the screen with the pictures above since stimulus sentences contained pointing signs that could be interpreted as direct pointing to one of the pictures.

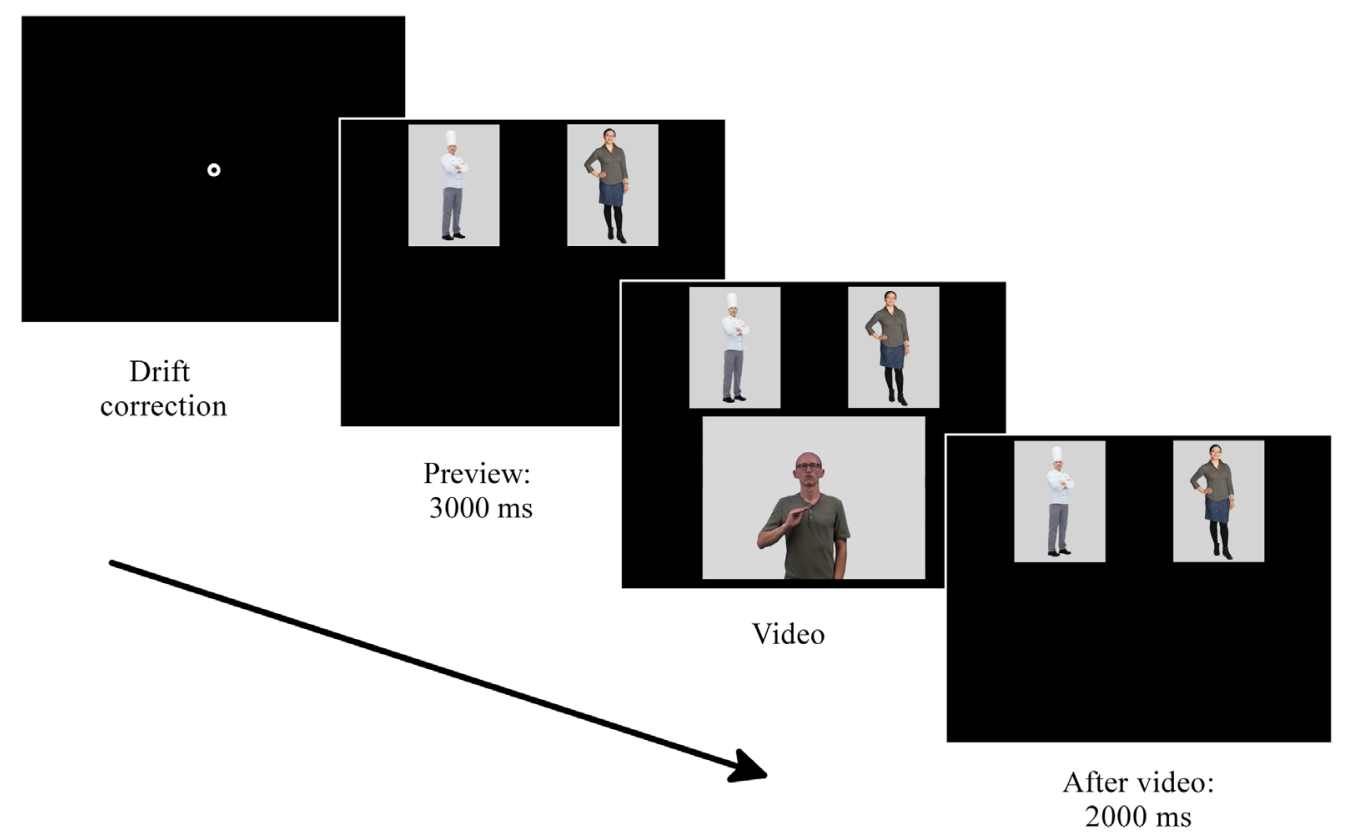

Figure 3.1 Structure of a single trial 
Eye movements were tracked and recorded with a sampling rate of $500 \mathrm{~Hz}$ using an EyeLink 1000 Desktop Mount (SR Research Ltd.) with a remote eye tracker. Data for each trial were recorded starting with the presentation of the pictures until these disappeared after the video presentation. Afterwards, the data were binned off-line into 40 ms sequences.

\subsection{Results}

The eye movement data were analyzed according to fixations to three areas of interest (AOIs), i.e., the video and the pictures of both referents. Mean proportion of target looking (PTL) was calculated for each condition and continuation type by dividing fixations to the target by the sum of fixations to all AOIs (target/(target + distractor + video)). The time course in Figure 3.2 presents target fixations prior and following the first fixation to one of the two presented pictures, i.e., the first fixation away from the video, following the beginning of the video. Therefore, the fixation onset was determined for each item and participant based on the fixation report exported using the Data Viewer Software (SR Research Ltd.). Visual inspection suggests a difference between conditions in the subject continuation type, but almost no difference for the object continuation type. 


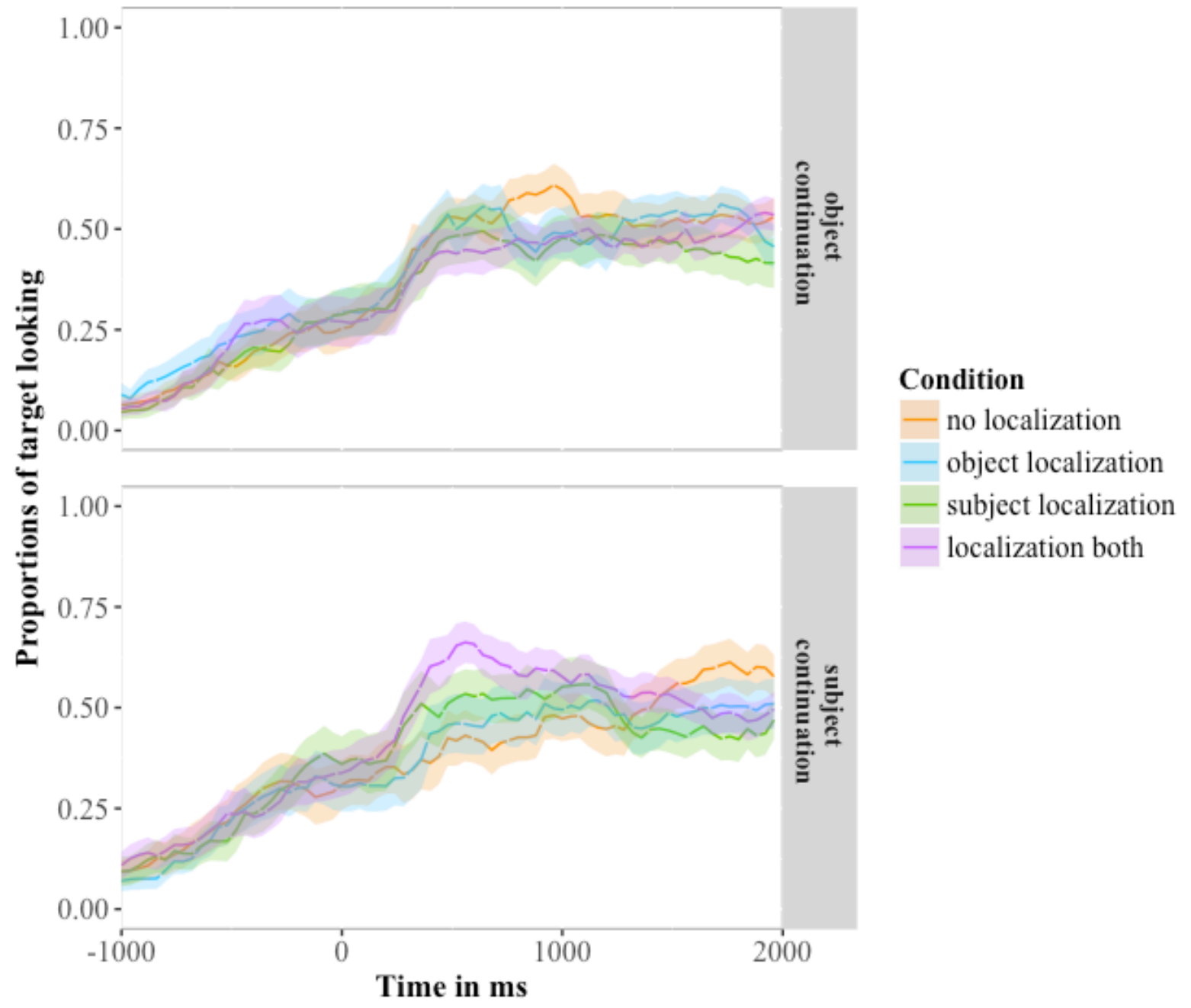

Figure 3.2 Mean proportion of target looking across time for each continuation type (from top to bottom: object continuation, subject continuation). The conditions are shown as lines (orange $=$ no localization, blue $=$ object localization, green $=$ subject localization, purple $=$ localization both) with $1000 \mathrm{~ms}$ previous and $2000 \mathrm{~ms}$ following the first fixation to one of the presented pictures.

For the analysis, mean proportion of target looking was examined across a time window of $1000 \mathrm{~ms}$ following the first fixation to one of the two presented pictures (Figure 3.3). The PTL was then empirical logit-transformed (Elog; see Appendix F for a figure displaying the Elog transformed data) using the EyetrackingR package (Dink \& Ferguson, 2015). 


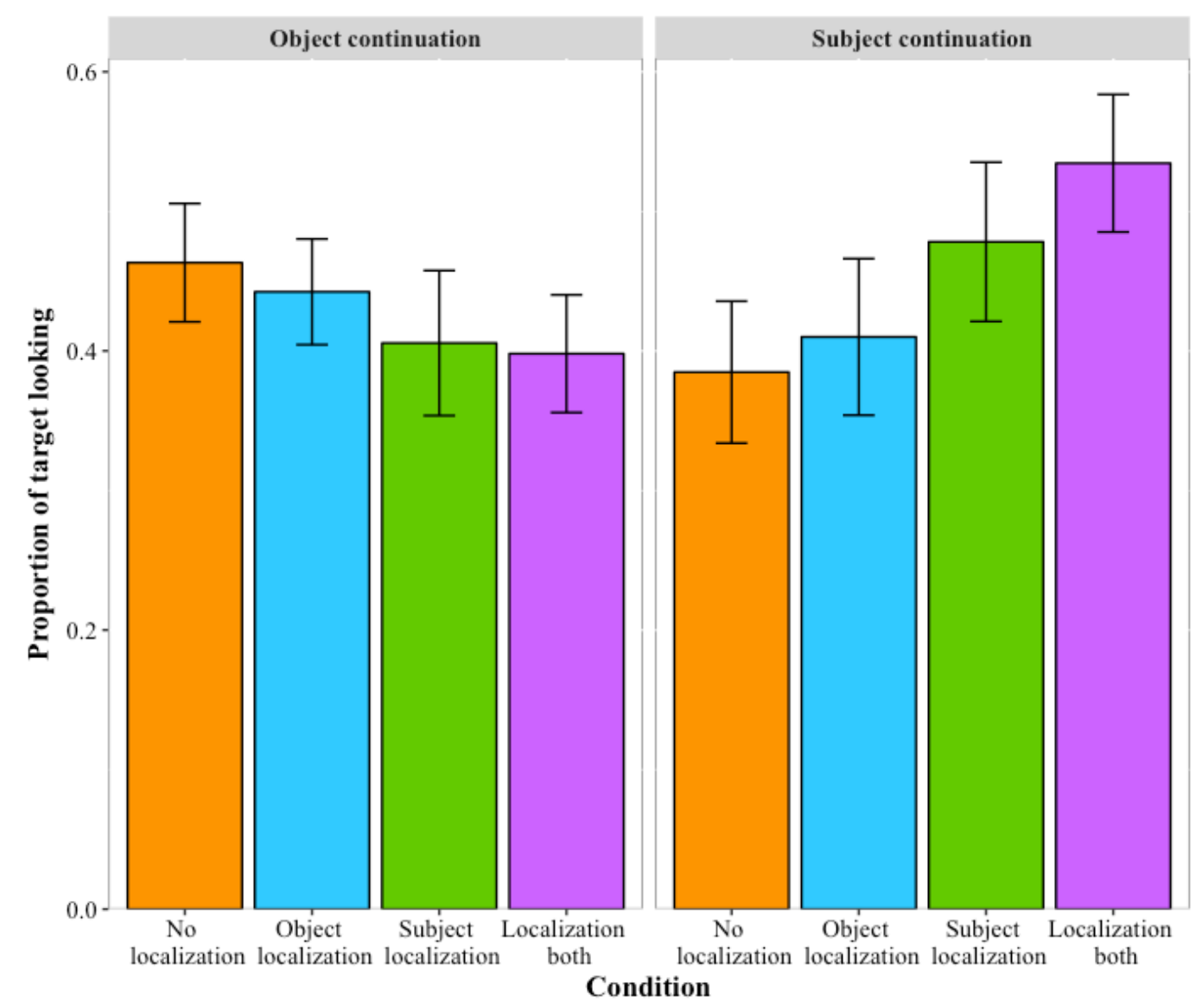

Figure 3.3 Mean proportion of target looking for object (left panel) and subject (right panel) continuation types and each condition (orange $=$ no localization, blue $=$ object localization, green $=$ subject localization, purple $=$ localization both) for $1000 \mathrm{~ms}$ following the first fixation to one of the presented pictures.

We fitted linear mixed-effects models using the lme4 package (Bates et al., 2014) in R (Version 3.4.2) with either fixed effects for condition (localization: none, object, subject, both), continuation type (object, subject) or the interaction of both as well as a full model. All models included random effects for items and participants. Comparing the models with maximum likelihood revealed the model using the interaction of condition and continuation type to be the best-fitting model $\left(\chi^{2}(4)=11.707 ; p=.02\right)$. The results of the best-fitting 
model (Table 3.1) indicate that subject continuations in connection with either the subject localizing or localizing both condition change the estimates most.

Table 3.1 Results of the best-fitting model for effects of condition, continuation type and their interaction presenting estimates, standard error (SE), t-value and lower $(2,5 \%)$ and upper $(97,5 \%)$ confidence interval (CI).

\begin{tabular}{llccccc}
\hline Fixed effects & & estimate & SE & t-value & lower CI & upper CI \\
\hline Condition & (Intercept) & -0.88 & 0.53 & -1.66 & -1.92 & 0.16 \\
& object & -0.12 & 0.46 & -0.25 & -1.03 & 0.79 \\
& subject & -0.41 & 0.46 & -0.90 & -1.32 & 0.49 \\
& both & -0.66 & 0.46 & -1.43 & -1.56 & 0.24 \\
Continuation type & subject & -0.56 & 0.48 & -1.17 & -1.49 & 0.37 \\
Interactions & object*subject & 0.59 & 0.66 & 0.89 & -0.71 & 1.89 \\
(condition*type) & subject*subject & 1.47 & 0.67 & 2.19 & 0.16 & 2.78 \\
& both*subject & 1.74 & 0.66 & 2.63 & 0.45 & 3.03 \\
\hline
\end{tabular}

This assumption is supported by additional mixed-effects analysis comparing a model for each condition using fixed effects for continuation type (object, subject) with a full model (Table 3.2). Again, all models included participants and items as random effects. Both models for the conditions subject localization $\left(\chi^{2}(1)=4.85 ; p=.03\right)$ and localization both $\left(\chi^{2}(1)=\right.$ $5.88 ; p=.02$ ) fit the data significantly better than the full model. In both conditions, there are more looks to the subject than to the object referent. The models for the conditions object localization $\left(\chi^{2}(1)=0.04 ; p>.1\right)$ and no localization $\left(\chi^{2}(1)=1.27 ; p>.1\right)$ did not lead to improvement. 
Table 3.2 Results of the models for each condition with fixed effects of continuation type and random effects of participants and items presenting estimates, standard error (SE), t-value and lower (2,5\%) and upper (97,5\%) confidence interval (CI).

\begin{tabular}{llccccc}
\hline Condition & & estimate & SE & t-value & lower CI & upper CI \\
\hline Localization & (Intercept) & -0.84 & 0.53 & -1.57 & -1.89 & 0.22 \\
none & Subject continuation & -0.54 & 0.48 & -1.13 & -1.48 & 0.40 \\
Localization & (Intercept) & -1.05 & 0.56 & -1.88 & -2.16 & 0.06 \\
object & Subject continuation & 0.09 & 0.45 & 0.19 & -0.80 & 0.97 \\
Localization & (Intercept) & -1.36 & 0.56 & -2.42 & -2.48 & -0.25 \\
subject & Subject continuation & 1.03 & 0.47 & 2.21 & 0.11 & 1.95 \\
Localization & (Intercept) & -1.49 & 0.51 & -2.94 & -2.50 & -0.49 \\
both & Subject continuation & 1.17 & 0.48 & 2.43 & 0.23 & 2.12 \\
\hline
\end{tabular}

\subsection{Discussion}

The current study investigates how overt localization of a discourse referent affects the processing of referential expressions and whether this correlates with the grammatical role of the localized referent. Therefore, we recorded participants' eye-movements while presenting two-sentence discourses containing two referents in a video and two pictures representing both referents. Within the discourses, the first sentence introduced two discourse referents with varying their localization, i.e., both, one of them or none, and the second sentence referred to one of the previous referents via a bare noun. Overall, results suggest that localization influences the processing of referential expressions. However, this effect only applies to the forms co-referential with the grammatical subject of the first sentence. 
The analyzed data suggest a conjoined effect of the factors localization and grammatical function on the processing of referential expressions. On the one hand, the overt localization seems to increase the prominence and activation of the antecedent. Nevertheless, there is no effect for the object continuation type and the object localization condition suggesting that localization itself is not sufficient to enhance processing. In contrast, effects for discourses continuing with the subject for conditions in which the subject was localized indicate an additional influence of the grammatical role of the referent. However, there are no effects observed in the condition of no overt localization and continuing with the subject referent suggesting that, in parallel to localization, this preference seems not to be sufficient to enhance processing on its own. Therefore, only an interaction of both factors can account for the observed effects. Hence, the processing of a referential expressions is facilitated if the antecedent is in the subject position and overtly localized at the same time. For spoken languages, Almor \& Eimas (2008) suggest that different factors, i.e., subjecthood, among others, add to the same effect, instead of eliminating the effect. The data of the current study provide evidence for a similar process in sign languages.

The observed effects can be explained by changes in the accessibility of antecedents modulating their activation pattern. Models of lexical access suggest that lexical entries need to exceed a specific activation threshold to be retrieved and that the activation of lexical entries varies based on lexical, semantic, syntactic or other pragmatic information (MarslenWilson \& Welsh, 1978; McClelland \& Rumelhart, 1981). In their framework, Greene et al. (1992) propose that comprehenders have a mental representation of entities, events and their relation as part of a discourse model. Within this model, each entity has an initial degree of accessibility varying based on syntactic, semantic and pragmatic information. This accessibility can change in subsequent discourse determined by new incoming syntactic and semantic properties (Greene et al., 1992). The antecedent of a referential expression is chosen 
based on the strongest activation (Gernsbacher, 1989; Greene et al., 1992). Applying this to the current study, the activation of lexical entries is affected by grammatical role and localization. Thus, referents that are localized and occur in subject position show increased activation leading to easier lexical retrieval when that referent is mentioned again in subsequent discourse since less additional activation is needed to exceed the retrieval threshold.

In spoken languages, prosodic focus, which is expressed by intonational accentuation of a constituent, focus particles or specific adverbs, is considered to be one of the factors highlighting a referent and leading to increased prominence contributing to facilitation in the processing of a referential expression co-referent with the focused constituent (Cutler et al., 1997). Similar observed effects in the current study allow for the assumption that overt localization increases the prominence of a constituent as well in the same way focus marker in spoken languages. Intonational marking in sign languages takes various forms such as increasing the duration or using a greater movement of a sign (Wilbur, 1999). Thus, instead of using one of these strategies, overt localization might be used to accentuate a referent. However, intonation is primarily described to be expressed by nonmanual articulators especially in the upper part of the face that bear multiple articulators used simultaneously (Sandler, 2012). Eye brow raise might be the most likely nonmanual marking used for intonation. On the one hand, it is the most prominent marker within the upper face, but on the other hand this marking is frequently used in various sentence types by adding it to a single manual sign or a whole phrase such as marking polar questions (Cecchetto, 2012) as well as relative and conditional clauses (Coulter, 1978; Wilbur \& Patschke, 1999). Often, focus marker in sign languages are accompanied by additional nonmanual marker, which is obligatoriy in some cases (Herrmann, 2014; Wilbur, 1999). Moreover, research on visual prosody accompanying spoken languages suggests that eye brow raise leads to increased 
prominence of a lexical item as well and often occurs in combination with prosodic focus intonation (Flecha-García, 2010; Srinivasan \& Massaro, 2003; Swerts \& Krahmer, 2008). It should be considered that brow raise in spoken language might be analyzed as a paralinguistic factor since meaning can be conveyed effectively even without this visual cue (Sandler, 2012). Nevertheless, it seems that brow raise affects the prominence of a lexical item irrespective of modality. Since manual and nonmanual marker are described to occur in combination quite frequently, it is reasonable to assume that adding brow raise to a localizing pointing sign might increase the prominence of a lexical item in sign languages as well. In general, the literature describes that manual and nonmanual marker are frequently combined when marking focus or topic suggesting that the effect of overt localization marker might be increased if accompanied by a nonmanual marker such as eye brow raise. It might be the case that localization in combination with eye brow raise would strengthen the effect of increasing a referent's prominence leading to higher activation of its mental concept. However, this is pure speculation because all kinds of nonmanuals were excluded in the stimulus material of the current study. Nevertheless, we were able to attribute observed effects partially to the manipulation of overt localization within the sentences. As a next step, stimulus sentences should contain systematic variation of eye brow raise and body leans since both factors were shown to be involved in focus marking across different sign languages (Herrmann, 2014; van der Kooij et al., 2006; Wilbur \& Patschke, 1998). This would allow to determine their influence on language processing in more detail.

\subsection{Conclusion}

This study provides evidence that overt localization increases the prominence and hence the accessibility of a discourse referent. Therefore, localization is analyzed as a focus marker similar to prosodic focus marking in spoken languages. However, localization itself 
seems not to be strong enough to increase the accessibility of a referent, but it correlates with the subject preference. Thus, both factors conjoined can facilitate accessing a referent's mental representation leading to faster and easier processing. In this respect, the current study is the first to determine the influence of manual localization on processing mechanisms.

However, this study cannot provide evidence for clarifying the role of nonmanuals since these were excluded during the preparation of the stimulus material. Therefore, it remains for future research to determine whether a specific nonmanual marking can add to the effect of overt localization. 


\title{
4 Phonological priming in German Sign Language: An eye tracking study using the Visual World Paradigm
}

\begin{abstract}
A number of studies provide evidence for a phonological priming effect in the recognition of single signs based on phonological parameters and that the specific phonological parameters modulated in the priming effect can influence the robustness of this effect. This eye tracking study on German Sign Language examined phonological priming effects at the sentence level, while varying the phonological relationship between prime-target sign pairs. We recorded participants' eye movements while presenting videos of sentences containing either related or unrelated prime-target sign pairs, and pictures of the target and an unrelated distractor. We observed a phonological priming effect for sign pairs sharing handshape and movement while differing in location parameter. Taken together, the data suggest a difference in the contribution of sign parameters to sign recognition and that sub-lexical features influence sign language processing.
\end{abstract}

Keywords: German Sign Language, eye tracking, Visual World Paradigm, sentence processing, phonological priming 


\subsection{Introduction}

Studies provide evidence for comparable lexical and sub-lexical organization of linguistic units from spoken and sign languages in the mental lexicon, even if modalityspecific differences do not allow for direct comparisons between the two language modalities. Similar to words in spoken language, signs can be decomposed into smaller meaningless phonological units. Stokoe et al. (Stokoe, 1960; Stokoe et al., 1965) were the first to describe specific hand configurations as phonemes in sign languages, i.e., in this case for American Sign Language (ASL). ${ }^{16}$ Each configuration can be specified by the major phonological parameters handshape, i.e., selection and flexion of (the) fingers, location, i.e., place of articulation of a sign in relation to the body, and movement, i.e., movements of the whole hand or arm as well as hand-internal movements including changes in handshape and palm orientation (Twilhaar \& van den Bogaerde, 2016). ${ }^{17}$

Furthermore, Stokoe and others (Klima \& Bellugi, 1979; Stokoe, 1960; Stokoe et al., 1965) highlight that parameters are produced simultaneously rather than sequentially. This suggests that single parameters might be processed in a parallel manner as well (Carreiras, 2010). However, succeeding studies revealed that parameters unfold over time, raising the possibility of sequential processing of sign language parameters (Liddell, 1984; Liddell \&

\footnotetext{
${ }^{16}$ Note that Stokoe and colleagues (Stokoe, 1960; Stokoe et al., 1965) did use the term chereme to introduce the concept of phonemes.

${ }^{17}$ Some researchers argued for including nonmanuals (Pfau \& Quer, 2010) and orientation, i.e., the direction of the palm, to the group of phonological parameters (Battison, 1974; Grosjean, 1980) while others defined orientation as a subcategory of handshape (Crasborn \& van der Kooij, 1997; Sandler \& Lillo-Martin, 2006).
} 
Johnson, 1989; Sandler, 1986). Indeed, we note that different parameters are available to the processor at different time points even in the transition phase between two signs (Hosemann et al., 2013). The question then arises how the different parameters influence the processing of signs. Here, the high temporal resolution of eye tracking paradigms provide us a privileged window into the recognition of signs as the sign unfolds. As we will show in the following sections, studies investigating different sign languages reveal that sign recognition, indeed, unfolds over time (Emmorey \& Corina, 1990) and that phonological parameters vary in their influence on sign recognition and processing.

\subsubsection{Effects of phonological parameters}

Studies have typically examined the status of sign language phonological parameters in sign recognition by varying the degree of overlap between two signs (see Table 4.1 for an overview). Summarizing the effects of the parameters investigated in these studies, the data highlight the special status of the location parameter compared to all other parameters. This parameter is acquired and identified first (Morgan et al., 2007; Siedlecki Jr \& Bonvillian, 1993), encoded more robustly in the mental lexicon (Orfanidou et al., 2009; Thompson et al., 2005) and leads to robust inhibition effects in picture-sign interference tasks (Baus et al., 2008, 2014). In contrast, the handshape and movement parameter show a more diverse and less coherent picture.

Table 4.1 Overview of studies examining effects of phonological parameters

\begin{tabular}{|l|l|l|l|}
\hline \multicolumn{1}{|c|}{ Authors } & Language & \multicolumn{1}{c|}{ Paradigm } & Results \\
\hline $\begin{array}{l}\text { Morgan et al. } \\
(2007)\end{array}$ & BSL & Case study & \\
\hline
\end{tabular}




\begin{tabular}{|c|c|c|c|}
\hline $\begin{array}{l}\text { Siedlecki Jr \& } \\
\text { Bonvillian (1993) }\end{array}$ & ASL & Corpus data & $\begin{array}{l}\text { Location is produced correctly } \\
\text { first, followed by movement and } \\
\text { handshape. }\end{array}$ \\
\hline $\begin{array}{l}\text { Grosjean (1980) } \\
\text { and Clark \& } \\
\text { Grosjean (1982) }\end{array}$ & ASL & Gating & $\begin{array}{l}\text { Location and handshape are } \\
\text { identified at about the same time } \\
\text { while movement is identified last. } \\
\text { All parameters are identified faster } \\
\text { when context is provided. }\end{array}$ \\
\hline $\begin{array}{l}\text { Emmorey \& Corina } \\
\text { (1990) }\end{array}$ & ASL & Gating & $\begin{array}{l}\text { Location is identified correctly first } \\
\text { followed by handshape and } \\
\text { movement. }\end{array}$ \\
\hline $\begin{array}{l}\text { Thompson et al. } \\
\text { (2005) }\end{array}$ & ASL & Tip-of-the-fingers & $\begin{array}{l}\text { Information about location and } \\
\text { handshape is retrieved from mental } \\
\text { lexicon while information about } \\
\text { movement is not retrieved. }\end{array}$ \\
\hline $\begin{array}{l}\text { Orfanidou et al. } \\
(2009)\end{array}$ & BSL & $\begin{array}{l}\text { Misperceptions in a sign } \\
\text { spotting task }\end{array}$ & $\begin{array}{l}\text { When identifying non-signs as real } \\
\text { signs, the location parameter is } \\
\text { rarely changed, the handshape } \\
\text { parameter is changed in some case } \\
\text { while the movement parameter is } \\
\text { frequently changed/adapted. }\end{array}$ \\
\hline $\begin{array}{l}\text { Gutiérrez et al. } \\
\text { (2012) }\end{array}$ & ASL & $\begin{array}{l}\text { ERP study on sentence } \\
\text { processing }\end{array}$ & $\begin{array}{l}\text { Stronger and broadly distributed } \\
\text { N400 effect is observed for signs } \\
\text { overlapping in their location } \\
\text { parameter compared to unrelated } \\
\text { signs. }\end{array}$ \\
\hline $\begin{array}{l}\text { Baus et al. (2008) } \\
\text { and Baus et al. } \\
\text { (2014) }\end{array}$ & LSC & Picture-sign interference & $\begin{array}{l}\text { Naming is facilitated for overlap in } \\
\text { only handshape, only movement or } \\
\text { both location and movement, but } \\
\text { naming is inhibited for overlap in } \\
\text { only location. }\end{array}$ \\
\hline
\end{tabular}

$\mathrm{BSL}=$ British Sign Language; ASL = American Sign Language; LSC = Catalan Sign Language

Taken together, the data presented in Table 1 suggest a hierarchy of the individual parameters and their impact on processing, such that the location parameter is prioritized in sign recognition followed by handshape and, lastly, movement. On the other hand, looking at 
the effects of combined parameters, the overlap in location and movement in signs leads to them being rated more similar and to increased facilitation effects in priming tasks (Brentari, 2006; Hildebrandt \& Corina, 2002). Thus, the parameters appear to have disparate influences depending on the degree of overlap in other parameters.

Priming studies examine how the response to a stimulus is affected by pre-exposure to a related stimulus. Thus, when a word is preceded by a phonologically related word, the recognition of the second word is typically faster than when it is preceded by an unrelated word (Hillinger, 1980; Slowiaczek \& Pisoni, 1986). However, there are differences in the speed or accuracy of word recognition based on the degree of overlap between words and the position of overlap within the word (Radeau et al., 1995; Slowiaczek et al., 2000; Slowiaczek \& Hamburger, 1992). Phonological priming has been attested for sign languages as well. Studies have shown that sign recognition is affected by a preceding phonologically related sign reflected in facilitation or inhibition in recognition of the target sign (see Table 4.2 for references).

In a lexical decision task, Corina \& Hildebrandt (2002) examined the effects of phonological form-based priming in ASL by presenting sign/sign and sign/non-sign pairs varying in their phonological overlap, i.e., unrelated, sharing the same location or sharing the same movement. The data showed that participants were slower to reject signs that overlapped phonologically with the prime. Using the same task, Carreiras et al. (2008) presented sign/sign and sign/non-sign pairs in Spanish Sign Language (LSE) sharing either the same handshape, the same location or being phonologically unrelated. They observed slower response times for signs sharing location, but no effect for shared handshape. Nonsigns showed faster response times for shared handshape, but no effect for shared location. In an ERP study, Gutiérrez et al. (2012) investigated sign recognition in LSE using similar 
stimuli and found higher N400 amplitude for signs with shared location, but a more negative going potential between 600 and $800 \mathrm{~ms}$ for non-signs with shared handshape. They explained this effect based on lexical competition. The identification of the location parameter itself is quite fast due the presence of fewer possible locations in general. However, this results in more competitors at each specific location and increases competition among them, which leads to inhibition reflected in slower reaction times and a higher N400 amplitude. In contrast, the recognition of the handshape parameter is more difficult due to the high degree of variability between the relatively large number of different handshapes. This results in increased processing costs reflected in a later ERP effect for non-signs, but leads to facilitation for signs with low-familiarity due to there being fewer competitors.

In contrast, ERP data on ASL showed reduced N400 amplitude for phonologically related prime-target pairs suggesting a facilitation effect in both implicit, i.e., reading words whose ASL translations are phonologically related, and explicit priming contexts (Meade et al., 2017, 2018). While Gutiérrez et al. (2012) investigated pairs overlapping in one parameter, Meade et al. (2018) presented pairs overlapping in two out of three parameters, which might explain the opposing direction of effects reported in these studies.

Similarly, Dye \& Shih (2006) investigated phonological priming effects in native signers of BSL for signs overlapping in one or more phonological parameters. They reported no effect for sign/sign pairs sharing handshape, but faster reaction times for sign/sign pairs sharing location and sharing both location and movement. The authors suggested that the effect was evoked by the interaction between signs in the lexicon. Thus, once a sign is activated, spreading activation leads to activation of related signs sharing the same location and movement parameter. Dye \& Shih (2006) concluded that lexical access was initiated by combined location and movement parameters for native signers. In contrast, Mayberry \& 
Witcher (2005) did not observe any effects for native signers using the same task, which they speculated might be due to ceiling effects in their baseline condition. However, they did not separately analyze minimal pairs with regards to particular parameters overlapping, such that averaging across the different minimal pair types may obscure potential differences. For German Sign Language (Deutsche Gebärdensprache: DGS), Hosemann (2015) reported an overall phonological priming effect in an ERP study, but did not present a more detailed analysis based on which parameters were shared by the minimal pairs.

Overall, the results presented in this section provide evidence for a phonological priming effect in sign languages. Even though the findings vary with regard to the influence of different parameters and the direction of effects, they show that signs sharing either location or both location and movement evoke different effects relative to other parameter combinations.

\subsubsection{Eye tracking studies in sign languages}

The eye tracking technique, i.e., recording participants' eye movements while they perceive a stimulus, is widely used in spoken language research to investigate processing of the phonological content of the input. Eye movements represent mental processes taking place during stimulus processing, i.e., eye-mind hypothesis (Just \& Carpenter, 1980). In sign language research, this method was initially used to investigate whether signers show systematic eye movements while perceiving or producing sign language (Hosemann, 2011; Thompson et al., 2006, 2009).

Up to now, only two studies have combined the eye tracking method for sign language research with the classical Visual World Paradigm (VWP) (Cooper, 1974; Tanenhaus et al., 
1995) to study sign language comprehension in adult signers. ${ }^{18}$ In the first study, Lieberman et al. (2015) presented participants with videos in ASL, displaying a single sign in the center of a screen surrounded by four pictures. In the unrelated condition, the target picture, i.e., the picture participants were asked to click on, was presented with three completely unrelated distractors. In phonologically related trials, distractors formed minimal pairs with the target sign, overlapping in two of three phonological parameters. Participants preferentially fixated phonological distractors compared to the unrelated distractors from $600 \mathrm{~ms}$ onwards: signers shifted their gaze towards the target picture even before the sign ends. The authors interpreted these results suggesting that phonological parameters were activated quite early and activated all possible candidates in the mental lexicon sharing same parameters, leading to lexical competition.

The second study was conducted by Thompson et al. (2013) in BSL. They simultaneously presented a video of a person signing sentences containing the carrier phrase $I$ see... followed by a target sign, centrally located on a screen surrounded by four pictures. Participants were asked to indicate whether the target sign matched one of the displayed pictures. Participants fixated target images whose corresponding BSL signs overlapped in both location and movement more than unrelated pictures. Additional analyses found differences in looking behavior between unrelated and location-movement overlap trials and a later effect for handshape-movement pairs. There was no difference across the time course for handshape-location pairs. The effect observed for location-movement pairs is in line with previous findings using different tasks and underlined the peculiar role of this parameter combination for lexical access in sign languages. The later effect of handshape-movement

\footnotetext{
18 Both studies examined semantic effects as well. However, these results are not presented here
} because they are not of interest for the purpose of the current study. 
pairs was interpreted as reflecting post-lexical processes where possible competitors are retrieved prior to the final decision on target identification. The authors concluded that lexical access in sign languages is driven by perceptual salience rather than the temporal order of accessible phonological parameters.

Both studies (see also Lieberman et al. (2017)) demonstrated that the VWP can be applied in sign language research as well. Lieberman et al. (2015) reported an overall effect of phonological competition for the processing of signs presented in isolation, whereas Thompson et al. (2013) presented a more fine-grained analysis based on groups of signs, embedded in a carrier phrase, sharing different combinations of phonological parameters. They showed that the overlap of location and movement parameters triggered different responses relative to other parameter combinations (see Table 4.2 for an overview of phonological priming and eye tracking studies). This is in line with the studies discussed in section 4.1.1.

Table 4.2 Overview of studies using different methods to investigate phonological priming in sign languages

\begin{tabular}{|c|c|c|c|c|c|c|c|}
\hline \multirow[b]{2}{*}{ Task } & \multirow[b]{2}{*}{ Language } & \multicolumn{6}{|c|}{ Results for overlap of } \\
\hline & & $\begin{array}{l}\text { Hand } \\
\text { shape }\end{array}$ & $\begin{array}{l}\text { Locat } \\
\text { ion }\end{array}$ & $\begin{array}{l}\text { Move } \\
\text { ment }\end{array}$ & $\begin{array}{l}\text { Hand- } \\
\text { shape \& } \\
\text { Location }\end{array}$ & $\begin{array}{l}\text { Location } \\
\text { \& Move- } \\
\text { ment }\end{array}$ & $\begin{array}{l}\text { Move- } \\
\text { ment \& } \\
\text { Location }\end{array}$ \\
\hline \multirow{3}{*}{$\begin{array}{l}\text { Lexical } \\
\text { decision }\end{array}$} & $\begin{array}{l}\text { ASL } \\
\text { (Corina \& } \\
\text { Hildebrandt } \\
\text {, 2002) }\end{array}$ & -- & $\begin{array}{l}\text { inhi- } \\
\text { bition }\end{array}$ & $\begin{array}{l}\text { inhi- } \\
\text { bition }\end{array}$ & -- & -- & -- \\
\hline & $\begin{array}{l}\text { ASL } \\
\text { (Mayberry } \\
\text { \& Witcher, } \\
\text { 2005) }\end{array}$ & -- & -- & -- & \multicolumn{3}{|c|}{ no effect (for native signers) } \\
\hline & $\begin{array}{l}\text { LSE } \\
\text { (Carreiras et } \\
\text { al., 2008) } \\
\end{array}$ & $\begin{array}{c}\text { no } \\
\text { effect }\end{array}$ & $\begin{array}{l}\text { inhi- } \\
\text { bition }\end{array}$ & -- & -- & -- & -- \\
\hline
\end{tabular}




\begin{tabular}{|c|c|c|c|c|c|c|c|}
\hline & $\begin{array}{l}\text { BSL } \\
\text { (Dye \& } \\
\text { Shih, 2006) }\end{array}$ & $\begin{array}{l}\text { no } \\
\text { effect }\end{array}$ & $\begin{array}{l}\text { facilit } \\
\text { ation }\end{array}$ & $\begin{array}{l}\text { no } \\
\text { effect }\end{array}$ & no effect & $\begin{array}{c}\text { facilitatio } \\
n\end{array}$ & no effect \\
\hline $\begin{array}{l}\text { ERP } \\
\text { (sign/sign } \\
\& \text { sign/non- } \\
\text { sign pairs) }\end{array}$ & $\begin{array}{l}\text { LSE } \\
\text { (Gutiérrez, } \\
\text { Müller, et } \\
\text { al., 2012) }\end{array}$ & $\begin{array}{l}\text { Inhi- } \\
\text { bition }\end{array}$ & $\begin{array}{l}\text { Inhi- } \\
\text { bition }\end{array}$ & -- & -- & -- & -- \\
\hline $\begin{array}{l}\text { ERP } \\
\text { (prime- } \\
\text { target pairs } \\
\text { within } \\
\text { sentences) }\end{array}$ & $\begin{array}{l}\text { DGS } \\
\text { (Hosemann, } \\
2015 \text { ) }\end{array}$ & -- & -- & -- & \multicolumn{3}{|c|}{$\begin{array}{c}\text { facilitation } \\
\text { (no analysis based on parameter } \\
\text { combinations) }\end{array}$} \\
\hline $\begin{array}{l}\text { ERP } \\
\text { (prime- } \\
\text { target pairs) }\end{array}$ & $\begin{array}{l}\text { ASL } \\
\text { (Meade et } \\
\text { al., 2018) }\end{array}$ & -- & -- & -- & \multicolumn{3}{|c|}{$\begin{array}{c}\text { facilitation } \\
\text { (no analysis based on parameter } \\
\text { combinations) }\end{array}$} \\
\hline \multirow{2}{*}{$\begin{array}{l}\text { Eye } \\
\text { tracking }\end{array}$} & $\begin{array}{l}\text { BSL } \\
\text { (Thompson } \\
\text { et al., 2013) }\end{array}$ & -- & -- & -- & $\begin{array}{c}\text { later } \\
\text { effect of } \\
\text { facilita- } \\
\text { tion }\end{array}$ & $\begin{array}{c}\text { facilita- } \\
\text { tion }\end{array}$ & no effect \\
\hline & $\begin{array}{l}\text { ASL } \\
\text { (Lieberman } \\
\text { et al., 2015) }\end{array}$ & -- & -- & -- & $\begin{array}{l}\text { facilita- } \\
\text { tion }\end{array}$ & inhibition & no effect \\
\hline
\end{tabular}

$\mathrm{ASL}=$ American Sign Language; LSE = Spanish Sign Language; BSL = British Sign Language; DGS = German

Sign Language

\subsubsection{The present study}

The current study investigated whether a phonological priming effect could be observed for DGS using a modified version of the VWP combined with the eye tracking technique. Moreover, we examined whether signs sharing specific phonological parameters differentially influenced processing reflected in systematic differences in participants' eyemovements in the parameter combinations presented. Extending previous work, especially by Thompson et al. (2013), we also examined whether eye tracking could be used to investigate processing at the sentence level using natural signed sentences, thereby examining natural language processing. Therefore, in contrast to Thompson et al. (2013) and Lieberman et al. (2015) our study presented prime-target sign pairs embedded in naturally signed sentences (see examples 1-3 below in section 4.2.2). Prime and target formed a minimal pair 
overlapping in two phonological parameters but differing in the third. ${ }^{19}$ This resulted in the following three priming parameter conditions labelled according to the overlap of parameters (Figure 4.1): (i) LOC+MOV condition, i.e., sharing location and movement; (ii) HS+MOV condition, i.e., sharing handshape and movement, and (iii) HS+LOC condition, i.e., sharing handshape and location. Additionally, a sentence containing an unrelated prime-target sign pair was constructed for each of the priming conditions. Each video was presented along with two pictures displaying the target and an unrelated distractor.

\section{Condition}

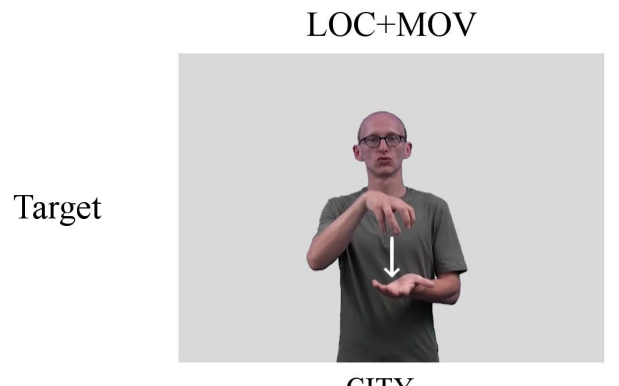

CITY

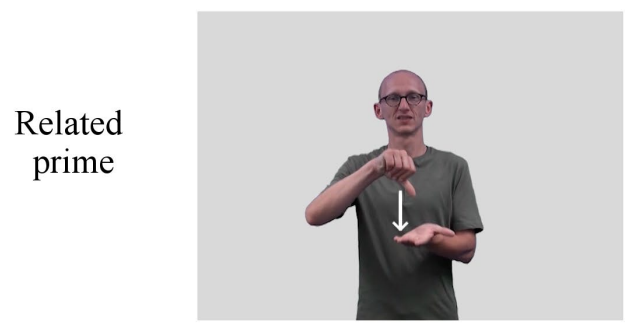

CENTRE

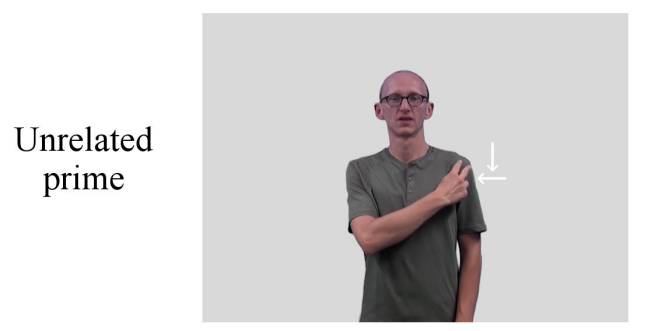

HOSPITAL

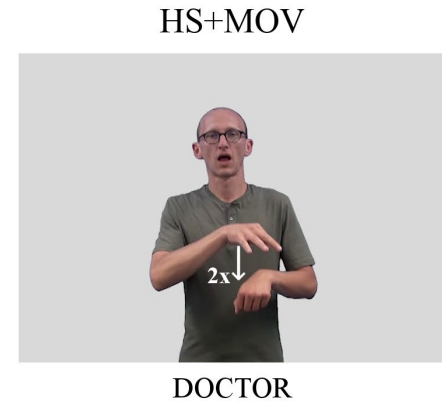

DOCTOR

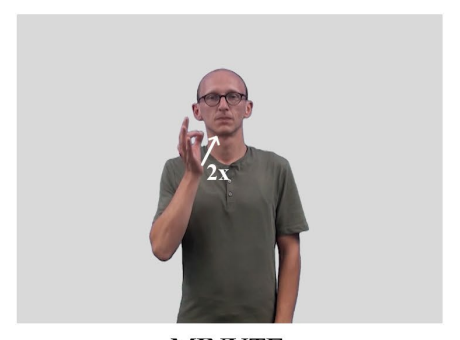

MINUTE

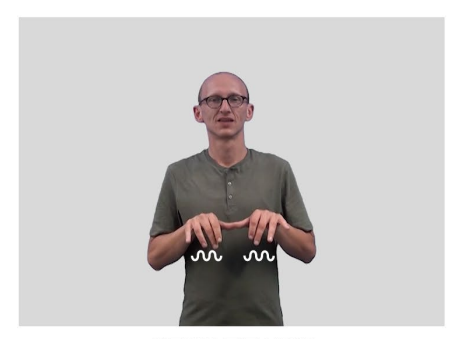

SECRETARY
HS+LOC
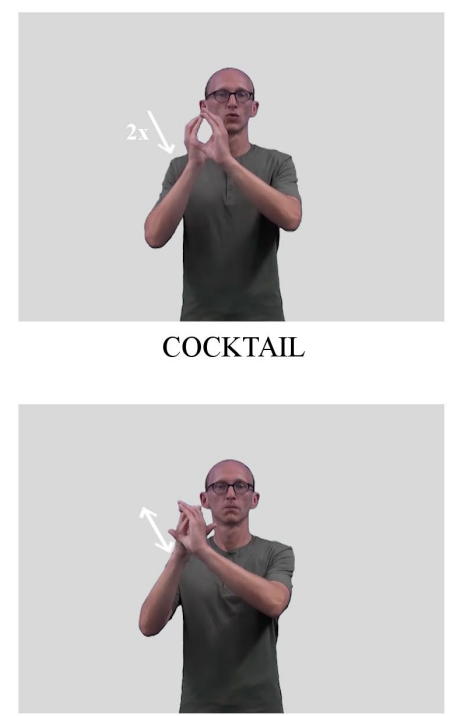

BUS

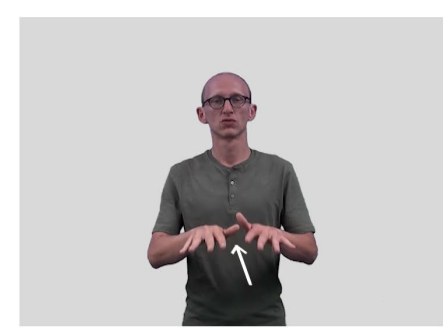

$\mathrm{BEACH}$

19 The definition of a minimal pair is adopted from research on spoken languages. To create a minimal pair in spoken languages, one phoneme in a word is substituted to create a new word with a different meaning. From a phonological point of view, this word pair only differs in a single phoneme (Crystal, 2008; Kenstowicz \& Kisseberth, 1979). 
Figure 4.1 Overview of prime-target sign pairs with examples from the stimuli: minimal pairs differing in handshape (left column), location (middle column) and movement parameter (right column). In the priming condition, the target (upper row) and the related prime (middle row) are combined. In the unrelated condition, the unrelated prime (bottom row) is combined with the target.

We recorded participants' eye movements as they looked at the pictures and videos presented simultaneously on a screen. We expected that participants will show increased fixations to the target in the primed condition compared to the unrelated condition for each parameter variation. We were particularly interested in how target fixations differ across the different parameter conditions, as this would inform us with regard to the influence of these parameters on sign recognition.

\subsection{Methods}

\subsubsection{Participants}

Twenty-six deaf native signers of DGS participated in the experiment. In total, three participants were excluded from the analysis either due to calibration failure (2) or due to extensive trackloss in the recorded data (1). The remaining 23 participants (11 female, 12 male) had a mean age of 33 years (range: 20 - 58 years) and at least high school education level. Twenty-one participants had deaf parents or exposure to DGS before the age of 5, whereas two were exposed to DGS at the ages of 8 and 15 years. However, these two had access to another sign language before and stated DGS to be their primary language of use for more than 20 years. Moreover, excluding these two participants did not lead to changes in the results. Participants were volunteers and got paid for their participation. Before starting the 
experiment, all subjects were informed about the procedure and gave written consent for their participation. The experiment was approved by the Ethics Committee of the Institute of Psychology.

\subsubsection{Material}

Ten sentences were constructed for each condition resulting in 60 stimulus sentences. However, for the HS+MOV condition, one minimal pair and its unrelated counterpart were excluded prior to the testing of the experiment because of an incorrect combination of signs in the stimulus video. Thus, 58 stimulus sentences remained for data analysis.

Sentences (see examples 1 to 3 below and Appendix G for a full list of the stimulus material) started with a locative, temporal, benefactive, adverbial or topic expression followed by the prime, an intervening sign, the target, and the predicate in sentence-final position (which might be accompanied by a modal or an adverbial). To avoid their occurrence in sentence-initial and sentence-final position, at least one sign preceded the prime and followed the target sign. A function sign, e.g., ALWAYS, OR, IX, was inserted between prime and target to give participants time to process the prime before seeing the target. Content words were avoided as intervening signs since they could evoke semantic associations and therefore interfere with the priming effect. In the priming conditions, all sentences contained a phonologically related minimal pair sharing two out of three phonological features and differing in the third feature as described above. In contrast, prime-target sign pairs in the unrelated conditions were completely phonologically unrelated, thus neither sharing any features in DGS nor orthographically in their German translation to avoid bimodal priming, i.e., priming in the second language (Hosemann et al., 2020; Kubus et al., 2015; Morford et al., 2011). Moreover, sign pairs in all conditions were not semantically related. Using the 
Noun Associations for German database by Melinger \& Weber (2006), we determined sematic associations between target signs and any preceding content sign. An analysis showed no significant difference between priming and unrelated conditions $(W=3, p>.1)$. Sentences were structurally identical for each prime-target sign pair in the priming and the unrelated condition. Targets were kept stable for both conditions while all other content words differed. Signs only occurred once as prime or target.

(1) LOC+MOV condition ${ }^{20}$

a. Priming

JUST IX1 CENTRE IX CITY VISIT.

'I just visited the center of the city.'

b. Unrelated

YESTERDAY IX1 HOSPITAL IX CITY GO-TO.

'Yesterday, I went to the hospital of the city.'

(2) HS+MOV condition

a. Priming

TWENTY MINUTES IX DOCTOR COME.

'In twenty minutes, a doctor will come.'

${ }^{20}$ Notational conventions: signs are glossed in small caps. A hyphen between signs indicates that more than one word is needed to gloss a single sign. Prime-target sign pairs are marked in bold. In the examples discussed below, ix 1 denotes the first person singular pronoun, ix is a demonstrative and poss 1 describes the first person possessive pronoun. Note that DGS uses SOV as its primary word order (Happ \& Vorköper, 2006). 
b. Unrelated

EVERY-DAY SECRETARY IX DOCTOR SUPPORT.

'Every day, the secretary supports the doctor.'

(3) HS+LOC condition

a. Priming

YESTERDAY EVENING POSS1 FRIEND BUS IX COCKTAIL DRINK.

'Yesterday evening, my friend drank a cocktail in the bus.'

b. Unrelated

HOLIDAYS IX POSS1 FATHER BEACH IX COCKTAIL ENJOY.

'During the holidays, my father enjoys a cocktail at the beach.'

The material was discussed and recorded with the help of a male deaf native signer of DGS using a Sony HDR-CX550VE camcorder. We did not control for the use of nonmanuals. However, the signer was instructed to reduce body movements such as body lean as well as to sign slower than their usual signing speed while maintaining a natural occurring speed. This was to allow participants increased time to process the presented stimuli while maintaining the duration of the videos more or less the same across all trials. Videos were cut and processed with the video editing software Adobe Premiere Pro CS6 such that the signer remained motionless for $1000 \mathrm{~ms}$ at the beginning and $1500 \mathrm{~ms}$ at the end of each video. Additionally, the background color of each video was changed to grey so that the background color of the video and the presented pictures was identical to avoid possible effects by different background colors. The videos were not modified further. Videos had a size of $732 \times 550$ pixels and a duration of $7720 \mathrm{~ms}$ - $12880 \mathrm{~ms}$ (mean duration: $10434 \mathrm{~ms}$ ). Paired samples t-test comparing target sign durations revealed no significant difference between 
priming and unrelated conditions as well as based on parameter conditions within the priming and unrelated conditions.

Additionally, a set of five practice sentences with the same structure was developed. Two sentences contained a minimal pair and three contained an unrelated sign pair. The minimal pairs used in the practice sentences were not used in the sentences during the experiment. These sentences were recorded and processed the same way as the stimulus material and were presented prior to the experiment to familiarize participants with the procedure.

Twenty-nine picture sets served as stimulus pictures. These were color photo-realistic images presented on a grey background with a size of 400x400 pixels. Two pictures were always presented at the same time. One of them showed the target and the other a distractor not related semantically, phonologically (neither in DGS nor in its written German translation) or in shape or size of the depicted objects. The same combinations of target and distractor images were shown in the priming and unrelated condition counterbalanced for their position onscreen. To ensure the unambiguity of the images independent of language modality, we conducted a picture-naming task with native speakers of German, in an online questionnaire using Google Forms (Google Inc.). Stimulus pictures for the present study and another eye tracking study (not relevant here) were tested in this questionnaire. For each noun, two images were presented to allow choosing the clearest picture resulting in 216 images in total divided into four lists containing 54 images each. Pictures for the same noun never occurred in the same list. Participants were instructed to name the image they see fast and spontaneously with one word. In total 101 participants (79 female, 22 male, mean age: 25 years) filled in the questionnaire. Eight participants were excluded prior to evaluation because 
they were not native speakers of German. For each noun, the answers to both of the presented pictures were compared. The image with the most exact and correct responses was chosen.

\subsubsection{Procedure}

Prior to testing, participants were informed about the eye tracking method and the procedure of the experiment. All explanations were given in DGS and written form to ensure that the information was accessible to all participants. Participants filled in a demographics form and gave written and signed consent prior to the start of the experiment. After finishing the experiment, participants were informed about the goal of the study.

Participants were seated at a table in front of a 17-inch computer screen with a resolution of 1280x1024 pixels. They were instructed to place their head on the chinrest to reduce head movements. The eye tracking camera was positioned right in front of the computer screen with 50-70 cm distance from the participant's face and adjusted manually to obtain a good image of the participant's eye. A PC computer was used to present the stimuli using the Eyelink Experiment Builder Software, Version 1.10.165 (SR Research Ltd.). At the beginning of the experiment, an introduction video in DGS explained the procedure followed by a short practice session to familiarize participants with the structure of the experiment. Participants could clarify remaining questions after the practice session. Then the actual experiment started with a nine-point calibration sequence. Additionally, a one-point drift correction, manually accepted by the experimenter, was performed before each trial. Stimuli were shown in two blocks with 29 trials each. Between the blocks, participants were given a break. In that time, they could relax their eyes and their body by removing the head from the chinrest. Therefore, the calibration sequence was repeated before continuing with the second block. 
Trials started with the presentation of the pictures on the top of the screen for $3000 \mathrm{~ms}$ (Figure 4.2). Following that, the stimulus video appeared on the screen below the pictures. The video disappeared after the stimulus video ended, i.e., when the hands of the signer were put down, and only the pictures remained on the screen for $2000 \mathrm{~ms}$. Then the next trial started automatically. Videos were presented at the bottom of the screen since sentences contained pointing signs that might be interpreted as direct pointings to the pictures and thus might interfere with the study. An Eyelink 1000 Desktop Mount (SR Research Ltd.) with a remote eye-tracker was used to automatically track and record the eye-movements with a sampling rate of $500 \mathrm{~Hz}$. Recording for each trial started with the initial presentation of the pictures and continued during the presentation of the video until the pictures disappeared. The data were binned offline into $40 \mathrm{~ms}$ sequences.

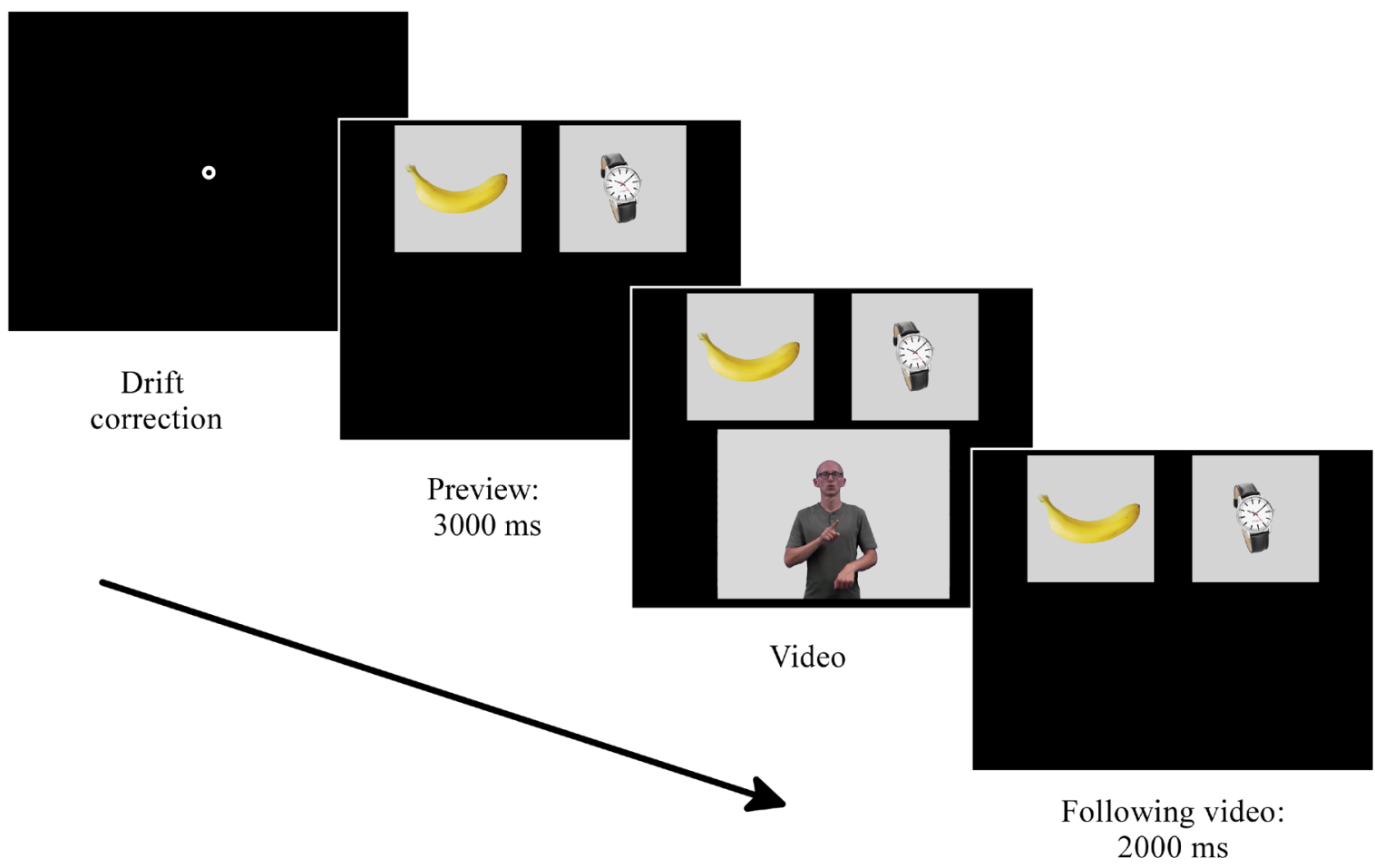

Figure 4.2 Structure of a single trial 
Four different randomization lists were prepared for stimulus presentation. Each list contained all sentences, only differing in order of presentation. For the first list, sentences were grouped into two blocks manually to avoid consecutive trials of the same minimal pair in the priming and unrelated condition. Each block contained ten trials per parameter condition - five in the priming and five in the unrelated condition. The position of the target was counterbalanced. For the second list, the same order of blocks was used, but positions of the images were reversed. In the third and fourth list, the order of the blocks was reversed such that the second block was presented first to account for order of presentation effects. Within each block the order of trials was randomized on-line by the experimental software with a maximum occurrence of two consecutive trials in the same condition (priming and unrelated).

\subsection{Results}

The eye movement data were analyzed according to fixations to three areas of interest (AOIs), i.e., the video and the pictures of target and distractor using the eyetrackingR package (Dink \& Ferguson, 2015) in R (Version 3.5.0). Mean proportion of fixations to the target (PTL) in each parameter condition comparing the priming and unrelated condition are plotted in Figure 4.3. The PTL is defined as fixations to the target divided by the sum of fixations to all AOIs (target/(target + distractor + video)). Since participants almost exclusively fixated the video during the video presentation, and only moved to the target sign upon presentation of this sign, earlier time windows were not considered in the analysis. Therefore, the time course does not represent the whole video, but is time-locked and aligned to the start of the target sign within each video. Since naturally signed sentences were used, the position of the target sign varied across the sentences and occurred at a different time point in each video. 
Therefore, the onset of the target sign had to be determined manually by two independent coders. Sign onset is defined as the first frame of a sign where target handshape and hand orientation reached their target location and hands are about to start the path movement toward their final position (Hosemann et al., 2013). Thus, the graph shows the time course starting from the onset of the target sign until the end of the video (range: $2080-3880 \mathrm{~ms}$; M $=2948 \mathrm{~ms}$ ) and $1500 \mathrm{~ms}$ following the offset of the video. As can be seen in the figure, looks to the target started to increase at around $1000 \mathrm{~ms}$ following target sign onset, especially for the HS+MOV parameter condition. Thus, participants started fixating the target picture only following the presentation of the target sign since these had a mean length of $528 \mathrm{~ms}$ (range: $160-880 \mathrm{~ms})$.

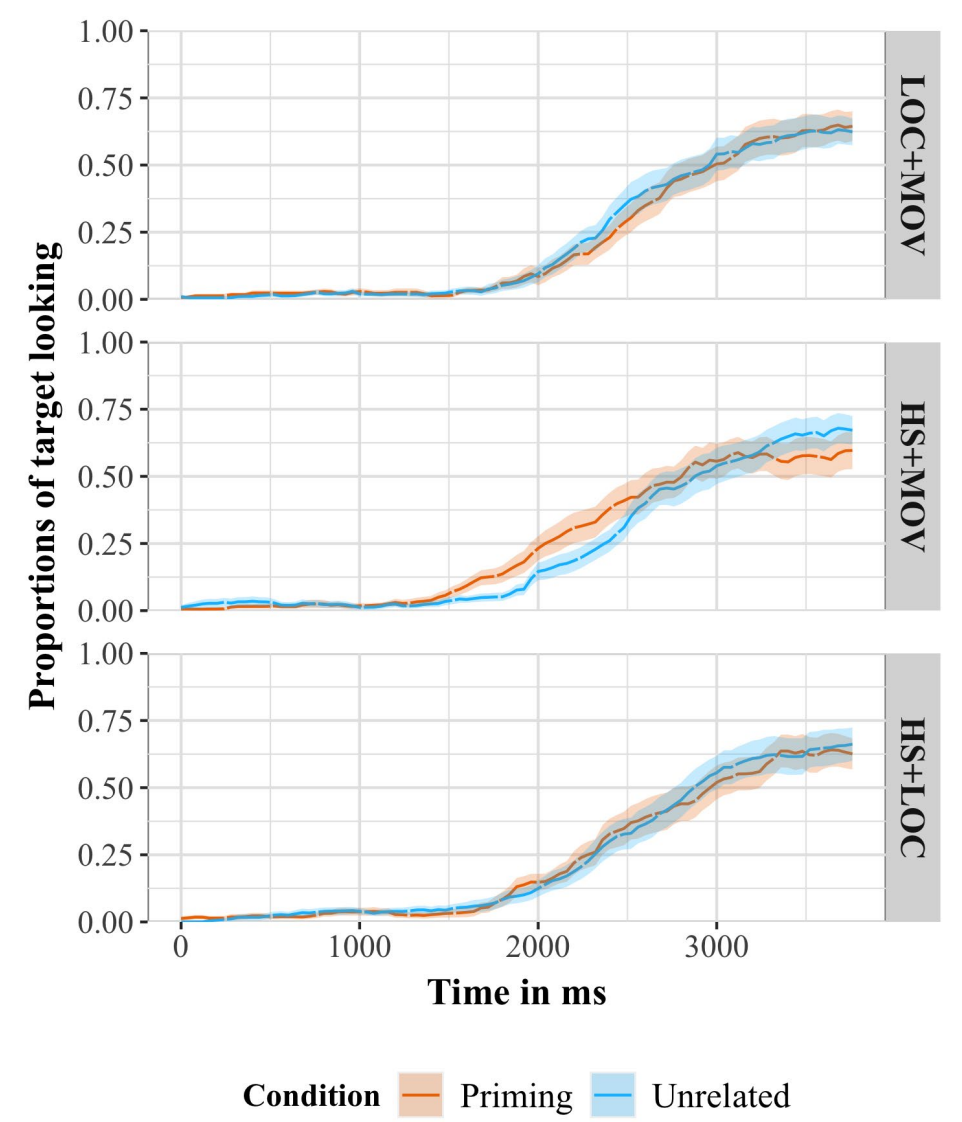

Figure 4.3 Mean proportion of target looking across time for each parameter condition (from top to bottom: location and movement, handshape and movement, handshape and location). 
The priming (orange) and unrelated (blue) conditions are shown as lines surrounded by shaded areas indicating standard error.

For the analysis, mean proportion of target looking was examined across a time window of $2000 \mathrm{~ms}$ (Hanna \& Tanenhaus, 2004), analyzing the data between $1000-3000 \mathrm{~ms}$ following target sign onset (Figure 4.4). The onset of the analysis window was chosen based on the duration of the longest target sign $(880 \mathrm{~ms})$, since the length of the target sign varied between the stimulus sentences, and adding a $100 \mathrm{~ms}$ for initiating a saccade to the target picture.

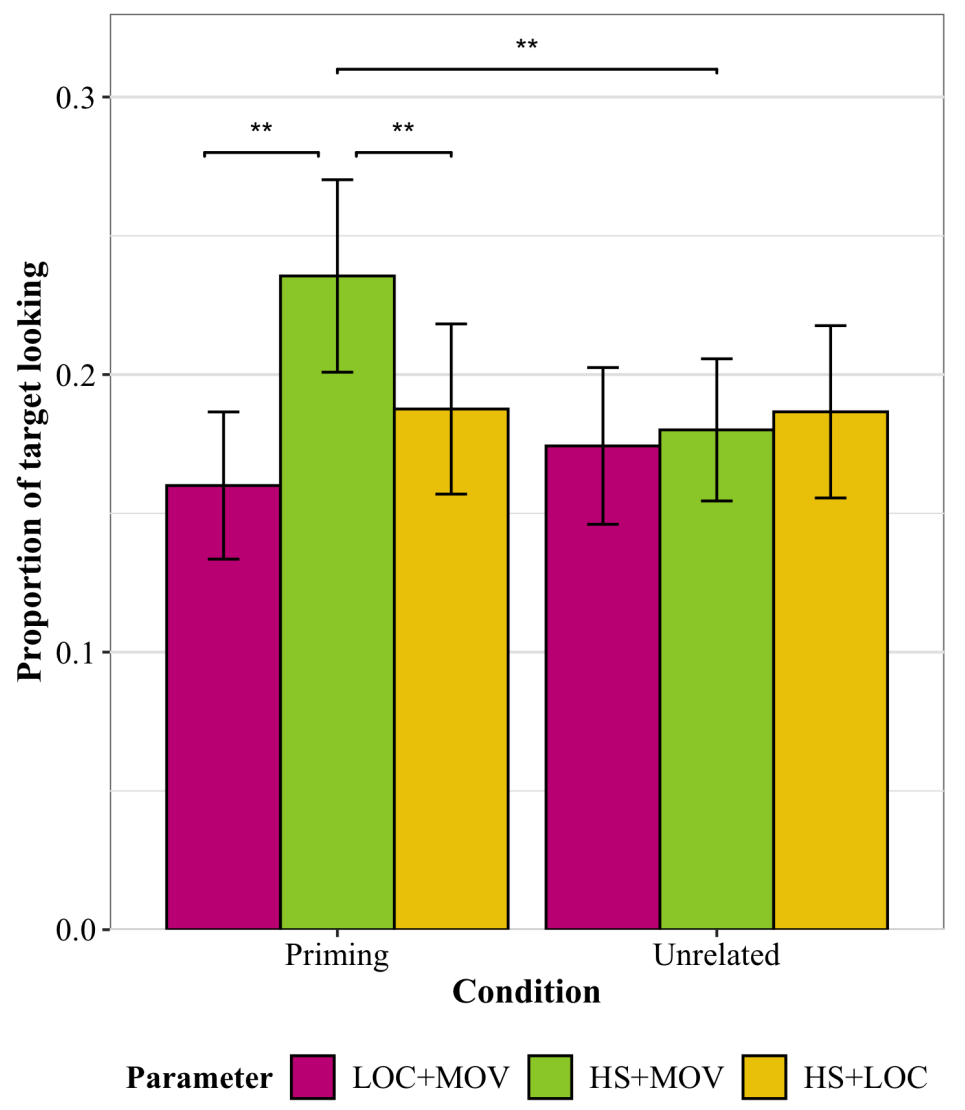

Figure 4.4 Mean proportion of target looking for priming and unrelated conditions for each parameter condition $($ pink $=$ location and movement; green $=$ handshape and movement, yellow $=$ handshape and location) between $1000-3000 \mathrm{~ms}$ following target sign onset. Error 
bars represent standard errors. Asterisks above bars indicate significance levels between bars: $* * \mathrm{p}<.01$

Running a 2x3 ANOVA with the factors condition (priming, unrelated) and parameter condition (LOC+MOV, HS+MOV, HS+LOC) revealed a main effect of parameter condition $(\mathrm{F}(2,44)=5.81 ; \mathrm{p}=.006)$ and an interaction between the factors $(\mathrm{F}(2,44)=4.99 ; \mathrm{p}=.011)$ Paired samples t-tests comparing priming and unrelated condition for each parameter revealed a significant difference in the HS+MOV parameter condition $(\mathrm{t}(22)=3.20 ; \mathrm{p}=.004)$ with higher PTL in the priming condition compared to the unrelated condition. There was no difference between conditions for LOC+MOV and HS+LOC parameter conditions $(\mathrm{p}>.1)$. Further, parameter conditions were compared using paired samples t-tests within priming and unrelated condition. This analysis revealed no difference between parameter conditions in the unrelated condition $(\mathrm{p}>.1)$. However, comparisons in the priming condition revealed a significant difference between $\mathrm{HS}+\mathrm{MOV}$ and $\mathrm{HS}+\mathrm{LOC}$ parameter conditions $(\mathrm{t}(22)=3.12 ; \mathrm{p}$ $=.005)$, as well as between HS+MOV and LOC+MOV parameter conditions $(\mathrm{t}(22)=3.39 ; \mathrm{p}$ $=.003)$ with, in both cases, more looks to the target in the HS+MOV parameter condition. Comparing HS+LOC and LOC+MOV parameter conditions showed a near-significant difference $(\mathrm{t}(22)=2.00 ; \mathrm{p}=.057)$ with more looks in the HS+LOC parameter condition. This resulted in a hierarchy for the different parameter conditions with the most looks in the HS + MOV parameter condition followed by HS+LOC and the fewest looks in the LOC+MOV parameter condition. Additional analyses on first fixation latency to the target AOI revealed a similar picture with the shortest latency in the HS+MOV parameter condition.

\subsection{Discussion}


The current study investigated whether a phonological priming effect at the sentence level in DGS can be observed and how variation in the phonological relatedness of sign pairs, i.e., which parameters they shared, influenced this effect. We examined this by recording participants' eye-movements while presenting them with videos, containing either related or unrelated prime-target sign pairs embedded in sentences, and two pictures, i.e., target and distractor, using a modified version of the VWP. Overall, results revealed a phonological priming effect. However, variation in phonological relatedness, i.e., the specific phonological parameters that overlap in a prime-target sign pair, had an impact on this effect in different ways since the parameters under investigation evoked distinct effects. Concerning the methodology, the experiment showed that priming effects in sign languages can be investigated by using eye tracking methodology.

The data presented in this study provide additional support for the hypothesis that phonological parameters impact sign processing differently. Sign processing is facilitated for sign that share the same handshape and movement. A more detailed analysis of the priming conditions suggested a taxonomy for the three parameter conditions with the HS+MOV parameter showing proportionally the most and the LOC+MOV parameter showing the fewest looks to the target picture, whereas the HS+LOC parameter fell in between, i.e., $\mathrm{HS}+\mathrm{MOV}>\mathrm{HS}+\mathrm{LOC}>\mathrm{LOC}+\mathrm{MOV}$. These findings are in line with Lieberman et al. (2015), who also observed similar effects for their phonological condition (as described in section 4.1). While the authors did not report differences within the phonological condition for the different groups of minimal pairs, analysis of the saccade latency to the target showed a marginal main effect for phonological parameter for a combined group of native and late signers. Participants were the fastest for minimal pairs sharing handshape and movement and the slowest when the pairs shared location and movement, whereas pairs sharing handshape and location fell in between (the data and the $\mathrm{R}$ code underlying this analysis are available at: 
DOI: $10.17605 / O S F . I O / X R B S E)$. Taken together, the results from both studies support the effect of a shared handshape and movement parameter. This is in contrast with previous studies that observe diverging effects for signs sharing location or location and movement (see section 4.1.1-4.1.3).

Nevertheless, the different approaches and results of previous research and our study are not contradictory, they are rather supporting each other. Carreiras et al. (2008) and Gutiérrez et al. (2012) both observed facilitation for overlap in handshape and inhibition for location overlap explaining this difference based on the concept of lexical competition. Overall, there are more possibilities for different handshapes than locations (for ASL (Liddell \& Johnson, 1989) and for DGS (Perniss, 2007; Pfau, 1997)) leading to fewer signs sharing the same handshape, but more signs sharing the same location. Thus, identifying the correct sign when overlapping in the location parameter requires more effort and time due to more competitors. In contrast, identification is faster and easier when there is an overlap in the handshape parameter because of fewer competitors. This offered a possible explanation of our results as well. Both conditions, where signs shared the same handshape, were faster than the condition that differed in handshape suggesting that an overlap in the handshape parameter enhances sign processing due to faster lexical processing. However, since sign pairs always shared two out of three parameters, not just one, this explanation has to be restricted and be seen with caution.

Running simulations in a computational model with a spreading activation architecture, Caselli \& Cohen-Goldberg (2014) discarded the notion of lexical competition to explain the differences between the handshape and location parameter. Using the computational model of word processing proposed by Chen \& Mirman (2012), they examined which modality-specific properties might account for the differences between the two 
parameters. Changes in the actual number of lexical neighbors did not produce the observed differences. Thus, it did not make a difference whether a sign had one or five neighbors. However, the model generated the expected results when implementing information about differences in timing and robustness of encoding reflected in the sub-lexical frequency. Timing refers to the point in time when a parameter is identified and, as described in section 4.1.1, the location parameter is identified before the handshape parameter (Emmorey \& Corina, 1990; Grosjean, 1980). Caselli \& Cohen-Goldberg (2014) concluded that the temporal order of parameter identification influenced a sign's perception due to stronger activation. When a parameter, i.e., sub-lexical feature, is activated, it sends its activation to all neighbors, i.e., signs sharing this feature. If this activation starts early, as in case of the location parameter, all competitors collect this activation for a longer time and are more robustly activated, which increases competition and leads to inhibition. In contrast, if activation starts later, as with the handshape parameter, there is less time to summate activation and competitors are less activated, which leads to less competition, i.e., facilitation. Caselli \& Cohen-Goldberg (2014) defined robustness of encoding based on the sub-lexical frequency, which they describe as similar to neighborhood density due to the definition of neighbors sharing a feature, leading to different levels of resting activation for each phonological parameter. Looking at previous studies, location indeed seems to be encoded differently since it is, e.g., less prone to tip-of-the-fingers (phenomena) and acquired earlier than handshape (Morgan et al., 2007; Siedlecki Jr \& Bonvillian, 1993; Thompson et al., 2005). Thus, if a parameter is encoded robustly, it has a higher sub-lexical frequency and a higher resting state level, which leads to strongly activated competitors and inhibition. On the other hand, a less robustly encoded feature has a lower resting state level so that competitors are less activated, which leads to facilitation. The inhibition effect for signs overlapping in the location parameter in previous literature suggests that location must be encoded more robustly while the facilitation effect for sharing the same handshape parameter suggests a less robust 
encoding for this parameter. According to Caselli \& Cohen-Goldberg (2014), both explanations were equally likely since varying either timing or sub-lexical frequency lead to the different effects for handshape and location observed in various experimental studies. Thus, sub-lexical properties accounted for the observed difference effects of handshape and location while lexical properties could not. Equally, we suggest that it is likely that sub-lexical properties played an important role in the effects reported in the current study. However, this raises the question whether the observed effect is based on the phonological parameters that differ or those that are shared between signs.

So far, the definition of the parameter conditions in the current study was based on the notion of minimal pairs in spoken languages. These were defined as word pairs that only differ in a single phoneme while all remaining phonemes are the same (Crystal, 2008; Kenstowicz \& Kisseberth, 1979). However, phonemes and phonological features in spoken languages are not directly comparable to phonological parameters in sign languages possibly leading to differences in defining minimal pairs in these two language modalities. For sign languages, Caselli \& Cohen-Goldberg (2014) propose a different account that is based on signs sharing one phoneme. In our study, we only included signs that share two out of three phonological parameters, but no signs overlapping a single parameter. Nevertheless, this taxonomy may allow for improved understanding of phonological parameters and their contribution to sign recognition revealing a new potential hierarchy based on the position of single parameters within the parameter combinations. The parameter occurring at the top was the handshape parameter suggesting that it should be seen as the most important cue in sign recognition. Then, the movement parameter followed, whereas the location parameter took the last position. Hence, the data suggest that sharing the same handshape parameter facilitated sign recognition whereas a shared location parameter seemed to slow down 
recognition. To verify the proposed hierarchy, future research is required to test signs overlapping in a single parameter.

\subsection{Conclusion}

From a methodological perspective, the study showed that eye tracking is an appropriate measure to investigate phonological priming effects for sign pairs embedded in natural sentences. Further, the current study provided additional evidence that sign languages are processed on a phonological level similar to spoken languages and contributed to the understanding of factors influencing sign recognition and processing. The data suggested differences in the contribution of phonological parameters to sign recognition. Caselli \& Cohen-Goldberg (2014) explanations based on the timing and sub-lexical frequency of particular phonological parameters could be applied to our data as well, suggesting that processing of sub-lexical features drive the effects observed in the current study.

Nevertheless, we should keep in mind that signs in each parameter conditions always shared two phonological parameters. Thus, to validate the suggested hierarchy for single parameters contributing to sign recognition, the current study needs to be repeated testing sign pairs sharing only one parameter. This would help to clarify the status of each phonological parameter. 



\section{General discussion}

This dissertation examines various aspects related to the processing of referential expressions (i.e., pronouns and full nouns) in DGS using different experimental methods. The presented studies all provide evidence for a modality-specific (Chapter 1) and modalityindependent (Chapter 2) strategy ensuring the comprehension of pronominal references in ambiguous contexts by analyzing obtained EEG data. Moreover, the influence of overt localization of discourse referents on their processing was investigated using the eye tracking method (Chapter 3) while an additional study tested the appropriateness of using that technique to investigate linguistic phenomena at the sentence level (Chapter 4).

The study presented in Chapter 1 examined the hypothesis that there is a default pattern that applies in the absence of overt localization during the assignment of discourse referents to referential locations. According to this pattern, right-handed signers link the first mentioned referent to the ipsilateral (right) and the second mentioned to the contralateral (left) side of the horizontal plane (Steinbach \& Onea, 2016). We presented participants with sentence sets in a semantic mismatch design where a pronoun and a semantically biased sign were incongruent in the mismatch conditions. Indeed, semantic mismatch conditions evoked an N400 compared to match conditions, thus providing evidence that signers automatically assign distinct and contrastive referential locations to different referents following the suggested default pattern. Additionally, mismatch conditions engendered an increased negativity across an early time window reflecting participants' sensitivity to violations of semantic/phonological expectations resembling the Phonological Mismatch Negativity effect. Moreover, a lateralization effect was observed with increased negativity in the left hemisphere for conditions containing a contralateral INDEX. This effect is interpreted as 
reflecting intrinsic attentional biases caused by the contralateral visual field, i.e., the INDEX occurs on the left side of the signer from the perspective of the perceiver and in their right visual field, thus causing the effect in the left hemisphere. Importantly, all effects were observed time-locked to the target handshape trigger position, i.e., when the target handshape of the sign is identifiable irrespective of orientation and location of the hand. This trigger position occurs in the transition phase between two signs and precedes the sign onset, which is defined as the moment when the target hand configuration is at the target location before the sign starts a movement, i.e., path or internal movement. Therefore, this study provides further evidence for transition phases to carry sufficient information to trigger a neurophysiological response, which is in line with Hosemann (2015), Hosemann et al. (2013) and Krebs (2017). Thus, studies investigating the neural basis of sign processing, including processing of referential expressions, should pay attention to the transition phase, which seems to play an important role in processing mechanisms.

In language comprehension, the first mention effect is a robust strategy that is used in understanding ambiguous contexts (Carreiras et al., 1995; Gernsbacher \& Hargreaves, 1988). Based on the results from Chapter 1 showing a default pattern in the assignment of discourse referent, Chapter 2 used the same stimulus material as in Chapter 1 to investigate the presence of a first mention effect in DGS. Neurophysiological responses were analyzed and timelocked to the recognition point of the direction of a pronominal INDEX sign presented at the beginning of the second sentence. The pronoun was either directed to the ipsilateral (right) side, i.e., referring to the first introduced referent, or to the contralateral (left) side, i.e., picking up the second referent. Conditions containing a contralateral INDEX evoked an N400 when compared to the ipsilateral INDEX suggesting an increase in processing for the former. Thus, participants seemed to expect the second sentence to continue with the first mentioned referent, i.e., the ipsilateral INDEX. However, this expectation is violated in the contralateral 
condition, which picked up the second referent. This violation is reflected in the observed N400 and speaks in favor of a first mention effect. Therefore, this study provides evidence for the first mention effect to be modality independent. However, it remains unclear whether the effect observed in this chapter could also be interpreted as a subject preference. In DGS basic word order, the first mentioned referent is assumed to be the subject of a sentence (Happ \& Vorköper, 2006). However, it is still an ongoing debate in theoretical sign language research whether the notions of subject and object can be applied to sign languages in the same way as for spoken languages. Therefore, we are cautious in calling the observed effect a subject preference. It is up to future researchers to disentangle the first mention effect and the subject preference in sign languages. Additionally, the effect in this chapter was observed in posterior regions on the right hemisphere. According to the classical assumption that language processing mainly involves the left hemisphere, this finding might be surprising. However, research has shown right hemispheric involvement in discourse processing, which includes semantic and pragmatic processes (Burgess \& Chiarello, 1996; Federmeier \& Kutas, 1999). In this respect, the right hemispheric effect might not be surprising at all since this study is tapping into discourse processing.

One factor that might affect the processing of referential expressions in sign languages is the overt localization of their antecedents. Using the eye tracking method, Chapter 3 examined the influence of overt localization on the processing of bare noun referential expressions and how this correlates with the grammatical function of the localized referent. Therefore, we presented participants with two-sentence discourses introducing two referents in the first sentence varying their overt localization. The second sentence started with a full noun co-referential with the first, i.e., the subject, or the second referent, i.e., the object, from the previous sentence. Analyzing proportions of target looking revealed effects for localizing both referents and only the subject for sentences continuing with the previous subject referent 
suggesting processing facilitation. Therefore, overt localization seems to increase the prominence of a referent, but the grammatical role of a referent seems to matter as well. The data can only be explained by an interaction of localization and the first mention preference presented in Chapter 2. Thus, processing of referential expressions co-referent with the previous subject is facilitated when both these factors come together. In contrast, localization itself is not sufficient to evoke this effect for expressions co-referential with the previous object. This modality-specific factor affects referential processing when combined the modality-independent first mention preference. However, the effect of localization itself, but also in combination with other factors needs to be determined in follow-up studies.

Since the eye tracking technique in combination with the Visual World Paradigm is quite new in sign language research, the study presented in Chapter 4 served to test the appropriateness of the experimental setting especially when presenting natural sentences, i.e. no manipulation of length, speed and nonmanuals during video editing. Therefore, we examined whether the phonological priming effect, which was shown for DGS before, can be observed with the intended setting and method as well. Thus, prime-target sign pairs with varied phonological relation, i.e., each combination of sharing two out of three phonological parameters, were embedded in natural sentences and presented in a video combined with a target and distractor picture. Analyzing the proportion of target looks revealed differences in the contribution of each parameter to sign recognition. The resulting hierarchy indicates that where sharing the same handshape can help facilitate sign recognition, sharing the same location parameter actually slows down the process. These differences seemed to be driven by sub-lexical features such as the point of identification of a specific parameter or their encoding in the mental lexicon (Caselli \& Cohen-Goldberg, 2014). However, it still needs to be tested in further studies whether this suggested hierarchy holds for DGS and possibly for other sign languages as well. Additionally, these data suggest that, at least on a phonological 
level, sign languages are processed just as spoken languages are with respect to phonological features/parameters. From the methodological perspective, this study confirmed that this combination of method and paradigm is a reliable setting for experimental sign language research and is particularly useful when investigating phenomena at sentence level.

Processes underlying language comprehension are extremely efficient and fast: only involving a couple of hundred milliseconds. Therefore, techniques that aim to examine the time course of these processes need to have a good temporal resolution, such as the neurophysiological EEG method or the eye tracking technique. Their measurements shed light on automatic processes which cannot be accessed by behavioral tasks. Even when participants are forced to give an immediate answer, by only allowing a short time window for answering, the results of behavioral tasks do not reflect the immediate automatic responses of the brain. As presented above, the behavioral judgement task accompanying the EEG study in Chapter 1 revealed no differences between the presented conditions. In general, judgement data are difficult to interpret since researchers cannot be sure about what exactly participants based their judgments on and subjective preferences might modify any of the effects measured (Schütze, 2016). In our judgement task, participants were required to rate the felicity/wellformedness of a presented sentence. However, their judgements could be based either on the syntactic structure, the semantic meaning of the sentences or on a combination of both. Therefore, we can only speculate about the reasons why all conditions obtained similar ratings. Moreover, judgement data require some kind of metalinguistic awareness, which might be difficult to access with respect to automatic linguistic processes. In contrast, the EEG method taps into these processes. The ERP data revealed a spatial default referent assignment suggesting that the participants were not aware of that default. Hence, the EEG method allowed us to glance at processes that would otherwise have remained covert. 
However, using the EEG and eye tracking method in studies on sign languages is somewhat challenging. The studies published so far show a lot of differences, especially with respect to the design of the stimulus material. The material used includes presenting sign-tosign (Kutas et al., 1987; Neville et al., 1997), presenting full sentences that are cross-spliced (Grosvald et al., 2012; Gutiérrez, Williams, et al., 2012) and using reduced nonmanuals (Hänel-Faulhaber et al., 2014; Hosemann, 2015; Hosemann et al., 2013). Similar to other studies, the stimulus material used in Chapter $1-3$ includes reduced nonmanuals. This might lead to an exclusion of certain cues that could be relevant for processing. At the same time, sentences without or reduced nonmanuals might appear artificial to participants, which might be an additional factor affecting processing. We decided to reduce the nonmanual markings since their influence on sign processing is not entirely clear at the moment. Therefore, our stimulus material might appear a bit artificial, but at least it is stable across all sentences. In contrast, keeping the natural nonmanual marking leads to a lot of variation between the sentences and, therefore, the interpretation of the data becomes a lot more difficult. Neither of these options are ideal, but there might be certain criteria which help decide in favor of one or the other strategy when considering the chosen stimulus material.

Moreover, this evokes even more difficulties during the assignment of the trigger positions used during data analyses. A lot of information is presented simultaneously using manual and nonmanual articulators but up to now it remains unclear how these different cues interact with each other and which ones are the most relevant for processing. Therefore, it is hard to determine an appropriate time point to be used for the ERP analysis. We based our trigger positions on Hosemann et al. (2013), who used the phonological properties of a sign to define the trigger positions. However, this did not seem to be the most appropriate definition for the aim of our studies looking into semantic and pragmatic processing because of the wide variety of other cues that could be necessary for triggering processing. This does not mean 
that we are questioning these definitions in general but rather asserting that we should adapt the trigger positions to best focus on the goal of a study. Prior research into predictive processing has shown that the transition phase between two signs contains information about the upcoming sign (Hosemann et al., 2013; Jantunen, 2010; ten Holt et al., 2009). The striking question is how much information in the transition phase is needed to trigger processing and how could this be determined. Moreover, the trigger position might vary with regard to certain properties of the stimulus material used (Hosemann et al., 2013). Another important factor in processing seems to be the availability of context information (Altmann \& Steedman, 1988; van Berkum et al., 1999). For sign languages, it needs to be determined what kind of information the context contributes to processing and how much context is needed to affect processing. All in all, there are a number of open questions that need to be answered on the way to establish standardized definitions for trigger positions which would help increase the comparability of EEG studies.

A similar problem arose during our early eye tracking studies where we used naturally signed sentences, we had to face several challenges when it came to analyzing the data. So far, eye tracking studies using the Visual World Paradigm have involved the manipulation of videos. In their study, Lieberman et al. (2015) presented isolated signs excluding the preceding and following transition phases so that the analysis was straight forward. Unfortunately, the study by Thompson et al. (2013), the first one to use sentences containing a carrier phrases followed by a target sign, does not report detailed information about the recording and editing of the videos. Another study by Lieberman et al. (2017) employed sentences as stimulus material as well, but they deleted additional frames in the video so that these had all the same length, which helped to facilitate the analysis considerably. This time, the videos contained transition phases, but they could deal with them quite easily since the sign preceding the target sign was the same across all sentences. Therefore, the authors 
included this phase into the time window of the target sign and controlled for co-articulation effects by doing so. This was not possible for our studies since the sign preceding the target differed in each case, and the sentences were of different lengths. Moreover, the target sign occurred at a different time point in each video, which would lead to blurred effects if the analysis would be time-locked to the start of each video. Therefore, the way of analyzing the data in the EEG studies was adapted and the starting point of the target sign in each video was determined manually. But again, the following questions remained: (i) which one of all possible starting points would be the most appropriate and (ii) how to decide for a specific time point and how to define it. Due to the predictive processing nature of language processing, a time point within the transition phase was chosen as the starting point again. However, it still remains an open issue whether the different length, i.e., difference in additional linguistic input following the target sign, might influence the detection of effects in general.

When using the eye tracking technique with deaf signers, characteristics regarding their vision abilities, especially the influence of peripheral vision, should be considered. It has been observed that deaf signers are more efficient at processing sentences visually due to the neural reorganization evoked since no auditory processing is required (Bavelier et al., 2000; Neville, 1990; Stivalet et al., 1998). Therefore, deaf signers show attention effects in ERP studies and faster response times in behavioral tasks to peripheral presented stimuli (Loke \& Song, 1991; Neville \& Lawson, 1987; Reynolds, 1993). These specific properties of vision in deaf signers are especially important during the implementation of the experimental setting regarding the size of video and pictures. Thus, the optimal relation between the perceptibility of all details in the stimulus materials and the necessity to engage an eye movement needs to be determined. Otherwise, it might be the case that no eye movements are observed due to either too big stimulus material that does not require any eye movement or too small videos 
requiring more attention and longer fixation times to perceive the content. Both cases might cover possible effects evoked by the content of the stimulus material. Moreover, the special characteristics of peripheral vision in deaf signers seem to develop during puberty. Deaf and hearing children do not differ in their performance on perceiving centrally or peripherally presented stimuli until around the age of 12 years old, but from then on deaf children show an increasing improvement in their peripheral vision until they reach the level expected of adults (Codina et al., 2011). Thus, the differences between testing deaf adults and children pose additional challenges when it comes to the design of an appropriate experimental setting for eye tracking studies.

Finally, the last challenge concerns the participants and their status as a monolingual deaf native signer. First of all, deaf subjects form a heterogeneous group of people having varying degrees of hearing loss. A person identifying as deaf does so based on their own cultural identity, and not based on any medical diagnosis (see Padden \& Humphries (1990) and Woodward (1972) for the distinction of Deaf vs. deaf). Thus, most Deaf people are actually hard of hearing. This might be a minor point for EEG and eye tracking studies, but more important for neuro-imaging studies assessing brain structures. More importantly here, is the status of a participant as being native and monolingual because bilingual participants seem to evoke different effects (Ardal et al., 1990; Kubus et al., 2015; Thierry \& Wu, 2007). But what does native mean with respect to a sign language? The ideal participant is a deaf signer that was born to deaf parents and thus learned sign language from the beginning. However, only $10 \%$ of deaf children are born to deaf parents, at least in Germany (Deutscher Gehörlosen-Bund e.V.). Thus, the preconditions of the remaining 90\% to acquire a sign language differ and show a high variability. Therefore, it is difficult to define 'native' under these conditions. In the studies presented in this thesis, signers were required to have acquired 
DGS as early as possible ${ }^{21}$ (before the age of 3 years in Chapter $1 \& 2$ and before the age of 5 years in Chapter $3 \& 4$ ). But again, a standardized regulation is required to allow for comparability across studies. Finally, the monolingual status of participants should be considered critically. In most cases, signers are not monolingual in the strict sense. From early childhood on, they are surrounded by the spoken language of the country the live in, i.e., signers of DGS are surrounded by written/spoken German, which they acquire quite early as well. In this respect, signers are bilingual across modalities. As was shown by Hosemann (2015), signers of DGS are primed with German as well. Thus, a possible interference effect due to the bilingual status should be considered during the planning and the preparation of experiments.

\section{Conclusion}

By conducting an ERP study, this thesis provides evidence for the presence of a default pattern of referent assignment and a first mention effect that both apply in ambiguous contexts, i.e., when discourse referents are not overtly linked to the space, and thus contribute to our understanding of referential expressions. Moreover, the first mention effect seems to interact with the overt localization of a subject referent facilitating processing investigated with the eye tracking method. Thus, this thesis presents supporting evidence for a first mention effect in DGS by using different experimental methods. From the sign language research perspective, this thesis supports the suitability of the eye tracking technique.

${ }^{21}$ A correlation of age and language ability is suggested by the critical period hypothesis proposed for spoken languages (Lenneberg, 1967). Thus, the earlier a language is acquired, the higher the probability to reach the level of a proficient language user. Various studies provide evidence for this correlation in sign languages as well (Mayberry, 1993; Newport, 1990). 
However, all of the studies presented herein leave a couple of open questions as well as pointers for areas that need improvement behind. Future research should test whether the observed default pattern holds for left-handed signers as well, to show whether this pattern is independent of the handedness of a signer. If this is not the case, and the default pattern differs between left- and right-handed signers, it would therefore need to be examined what happens in a conversation between opposite handed signers, especially in ambiguous contexts. More generally, the role of nonmanuals and their correlation with manually conveyed information has to be determined in more detail. First evidence that nonmanuals do trigger processing is shown by Krebs (2017), but more systematic investigations are needed. Additionally, the role of other factors (i.e., verb semantics) that might apply during the processing of referential expressions need to be investigated. In the end, researchers need to know more about word order and its variations in general, as well as about the effects observed in sign language specific constructions, e.g., topic or classifier constructions, to allow for generalizations to be made about observed effects. Therefore, combining experimental and corpus data would allow for more comprehensive natural sign language data. 



\section{References}

Aarons, D. (1994). Aspects of the syntax of American Sign Language [Doctoral dissertation]. Boston University.

Almor, A. (1999). Noun-phrase anaphora and focus: The informational load hypothesis. Psychological Review, 106(4), 748-765. https://doi.org/10.1037//0033-295x.106.4.748

Almor, A., \& Eimas, P. D. (2008). Focus and noun phrase anaphors in spoken language comprehension. Language and Cognitive Processes, 23(2), 201-225. https://doi.org/10.1080/01690960701330936

Almor, A., \& Nair, V. A. (2007). The Form of Referential Expressions in Discourse. Language and Linguistics Compass, 1(1-2), 84-99. https://doi.org/10.1111/j.1749818X.2007.00009.x

Altmann, G., \& Steedman, M. (1988). Interaction with context during human sentence processing. Cognition, 30(3), 191-238. https://doi.org/10.1016/0010-0277(88)90020-0

Andreassi, J. L., Okamura, H., \& Stern, M. (1975). Hemispheric asymmetries in the visual cortical evoked potential as a function of stimulus location. Psychophysiology, 12(5), 541-546. https://doi.org/10.1111/j.1469-8986.1975.tb00043.x

Ardal, S., Donald, M. W., Meuter, R., Muldrew, S., \& Luce, M. (1990). Brain responses to semantic incongruity in bilinguals. Brain and Language, 39(2), 187-205. https://doi.org/10.1016/0093-934X(90)90011-5

Ariel, M. (1988). Referring and accessibility. Journal of Linguistics, 24(1), 65-87. https://doi.org/10.1017/S0022226700011567

Ariel, M. (1990). Accessing noun-phrase antecedents. Routledge.

Ariel, M. (2001). Accessibility theory: An overview. In T. J. Sanders, J. Schilperoord, \& W. Spooren (Eds.), Text representation: Linguistic and psycholinguistic aspects (Vol. 8, pp. 29-87). John Benjamins Publishing Company.

Arnold, J. E. (1998). Reference form and discourse patterns [Doctoral dissertation, Stanford University]. http://www.unc.edu/ jarnold/papers/diss/fulldissertation.doc

Arnold, J. E., Eisenband, J. G., Brown-Schmidt, S., \& Trueswell, J. C. (2000). The rapid use of gender information: Evidence of the time course of pronoun resolution from eyetracking. Cognition, 76(1), B13-B26. https://doi.org/10.1016/s00100277(00)00073-1

Aronoff, M., Meir, I., \& Sandler, W. (2005). The Paradox of Sign Language Morphology. 
Language, 81(2), 301-344.

Bahan, B., Kegl, J., MacLaughlin, D., \& Neidle, C. (1995). Convergent evidence for the structure of determiner phrases in American Sign Language. In G. Leslie, D. Hardison, \& R. Westmoreland (Eds.), FLSM VI: Proceedings of the Sixth Annual Meeting of the Formal Linguistics Society of Mid-America. Volume Two: Syntax II \& Semantics/Pragmatics (Vol. 2, pp. 1-12). Indiana University Linguistics Club. http://www.academia.edu/download/30909814/flsm.pdf

Barberà, G. (2012). The Meaning of Space in Sign Language. Reference, Specificity and Structure in Catalan Sign Language Discourse. De Gruyter Mouton.

Bates, D., Mächler, M., Bolker, B., \& Walker, S. (2014). Fitting linear mixed-effects models using lme4. ArXiv Preprint ArXiv: 1406.5823.

Battison, R. (1974). Phonological deletion in American Sign Language. Sign Language Studies, 5(1), 1-19. https://doi.org/10.1353/sls.1974.0005

Baus, C., Gutiérrez, E., \& Carreiras, M. (2014). The role of syllables in sign language production. Frontiers in Psychology, 5(1254). https://doi.org/10.3389/fpsyg.2014.01254

Baus, C., Gutiérrez-Sigut, E., Quer, J., \& Carreiras, M. (2008). Lexical access in Catalan Signed Language (LSC) production. Cognition, 108(3), 856-865. https://doi.org/10.1016/j.cognition.2008.05.012

Bavelier, D., Tomann, A., Hutton, C., Mitchell, T., Corina, D. P., Liu, G., \& Neville, H. (2000). Visual attention to the periphery is enhanced in congenitally deaf individuals. Journal of Neuroscience, 20(17), RC93-RC93.

Birch, S. L., Albrecht, J. E., \& Myers, J. L. (2000). Syntactic focusing structures influence discourse processing. Discourse Processes, 30(3), 285-304. https://doi.org/10.1207/s15326950dp3003_4

Bornkessel-Schlesewsky, I., \& Schlesewsky, M. (2009). Processing syntax and morphology: A neurocognitive perspective (Vol. 6). Oxford University Press.

Bouchard, D., \& Dubuisson, C. (1995). Grammar, order \& position of wh-signs in Quebec Sign Language. Sign Language Studies, 87(1), 99-139. https://doi.org/10.1353/sls.1995.0007

Boyes Braem, P. (1995). Eine Einführung in die Gebärdensprache und ihre Erforschung. Signum.

Brentari, D. (1998). A Prosodic Model of Sign Language Phonology. MIT Press.

Brentari, D. (2006). Effects of language modality on word segmentation: An experimental 
study of phonological factors in a sign language. In L. Goldstein, D. H. Whalen, \& C. T. Best (Eds.), Laboratory Phonology (Vol. 8, pp. 155-164). De Gruyter Mouton.

Burgess, C., \& Chiarello, C. (1996). Neurocognitive mechanisms underlying metaphor comprehension and other figurative language. Metaphor and Symbol, 11(1), 67-84. https://doi.org/10.1207/s15327868ms1101_4

Capek, C. M., Grossi, G., Newman, A. J., McBurney, S. L., Corina, D. P., Röder, B., \& Neville, H. J. (2009). Brain systems mediating semantic and syntactic processing in deaf native signers: Biological invariance and modality specificity. Proceedings of the National Academy of Sciences, 106(21), 8784-8789.

https://doi.org/10.1073/pnas.0809609106

Capek, C. M., MacSweeney, M., Woll, B., Waters, D., McGuire, P. K., David, A. S., Brammer, M. J., \& Campbell, R. (2008). Cortical circuits for silent speechreading in deaf and hearing people. Neuropsychologia, 46(5), 1233-1241.

Carreiras, M. (2010). Sign Language Processing. Language and Linguistics Compass, 4(7), 430-444. https://doi.org/10.1111/j.1749-818X.2010.00192.X

Carreiras, M., Gernsbacher, M. A., \& Villa, V. (1995). The advantage of first mention in Spanish. Psychonomic Bulletin \& Review, 2(1), 124-129. https://doi.org/10.1037/e665412011-201

Carreiras, M., Gutiérrez-Sigut, E., Baquero, S., \& Corina, D. P. (2008). Lexical processing in Spanish Sign Language (LSE). Journal of Memory and Language, 58(1), 100-122. https://doi.org/10.1016/j.jml.2007.05.004

Caselli, N. K., \& Cohen-Goldberg, A. M. (2014). Lexical access in sign language: A computational model. Frontiers in Psychology, 5(428). https://doi.org/10.3389/fpsyg.2014.00428

Cecchetto, C. (2012). Sentence types. In R. Pfau, M. Steinbach, \& B. Woll (Eds.), Sign Language: An International Handbook (pp. 292-315). De Gruyter Mouton.

Chen, Q., \& Mirman, D. (2012). Competition and cooperation among similar representations: Toward a unified account of facilitative and inhibitory effects of lexical neighbors. Psychological Review, 119(2), 417-430. https://doi.org/10.1037/a0027175

Clark, L. E., \& Grosjean, F. (1982). Sign recognition processes in American Sign Language: The effect of context. Language and Speech, 25(4), 325-340. https://doi.org/10.1177/002383098202500402

Codina, C., Buckley, D., Port, M., \& Pascalis, O. (2011). Deaf and hearing children: A comparison of peripheral vision development. Developmental Science, 14(4), 725- 
737.

Connolly, J. F., \& Phillips, N. A. (1994). Event-related potential components reflect phonological and semantic processing of the terminal word of spoken sentences. Journal of Cognitive Neuroscience, 6(3), 256-266. https://doi.org/10.1162/jocn.1994.6.3.256

Connolly, J. F., Phillips, N. A., Stewart, S. H., \& Brake, W. G. (1992). Event-related potential sensitivity to acoustic and semantic properties of terminal words in sentences. Brain and Language, 43(1), 1-18. https://doi.org/10.1016/0093-934x(92)90018-a

Connolly, J. F., Stewart, S. H., \& Phillips, N. A. (1990). The effects of processing requirements on neurophysiological responses to spoken sentences. Brain and Language, 39(2), 302-318. https://doi.org/10.1016/0093-934X(90)90016-a

Cooper, R. M. (1974). The control of eye fixation by the meaning of spoken language: A new methodology for the real-time investigation of speech perception, memory, and language processing. Cognitive Psychology, 6(1), 84-107. https://doi.org/10.1016/0010-0285(74)90005-x

Corina, D. P., \& Hildebrandt, U. C. (2002). Psycholinguistic investigations of phonological structure in ASL. In R. P. Meier, K. Cormier, \& D. Quinto-Pozos (Eds.), Modality and structure in signed and spoken languages (pp. 88-111). Cambridge University Press.

Cormier, K. (2005). Exclusive pronouns in American Sign Language. In E. Filimonova (Ed.), Clusivity: Typology and Case Studies of Inclusive-Exclusive Distinction (Vol. 63, pp. 241-268). Benjamins.

Cormier, K. (2007). Do All Pronouns Point? Indexicality of First Person Plural Pronouns in BSL and ASL. In P. Perniss, R. Pfau, \& M. Steinbach (Eds.), Visible Variation: Comparative Studies on Sign Language Structure (Vol. 188, pp. 63-101). De Gruyter Mouton.

Cormier, K. (2012). Pronouns. In R. Pfau, M. Steinbach, \& B. Woll (Eds.), Sign Language: An International Handbook (pp. 227-244). De Gruyter Mouton.

Cormier, K., Schembri, A., \& Woll, B. (2013). Pronouns and pointing in sign languages. Lingua, 137, 230-247. https://doi.org/10.1016/j.lingua.2013.09.010

Coulter, G. (1978). Raised Brows and Wrinkled Noses: The Grammatical Function of Facial Expression in Relative Clauses and Related Constructions. In F. Caccamise \& D. Hicks (Eds.), American Sign Language in a Bilingual, Biclutural Conext. Proceedings of the second National Symposium on Sign Language Research and Teaching (pp. 6574). 
Cowles, H. W., Walenski, M., \& Kluender, R. (2007). Linguistic and cognitive prominence in anaphor resolution: Topic, contrastive focus and pronouns. Topoi, 26(1), 3-18. https://doi.org/10.1007/s11245-006-9004-6

Crasborn, O., \& van der Kooij, E. (1997). Relative orientation in sign language phonology. In J. Coerts \& H. de Hoop (Eds.), Linguistics in the Netherlands 1997 (pp. 37-48). John Benjamins Publishing Company.

Crawley, R. A., \& Stevenson, R. J. (1990). Reference in single sentences and in texts. Journal of Psycholinguistic Research, 19(3), 191-210. https://doi.org/10.1007/BF01077416

Crawley, R. A., Stevenson, R. J., \& Kleinman, D. (1990). The use of heuristic strategies in the interpretation of pronouns. Journal of Psycholinguistic Research, 19(4), 245-264. https://doi.org/10.1007/bf01077259

Crystal, D. (2008). Dictionary of Linguistics and Phonetics (6th ed.). Blackwell Publishing.

Cutler, A., Dahan, D., \& Van Donselaar, W. (1997). Prosody in the comprehension of spoken language: A literature review. Language and Speech, 40(2), 141-201. https://doi.org/10.1177/002383099704000203

Cutler, A., \& Fodor, J. A. (1979). Semantic focus and sentence comprehension. Cognition, 7(1), 49-59. https://doi.org/10.1016/0010-0277(79)90010-6

D’Arcy, R. C., Connolly, J. F., \& Crocker, S. F. (2000). Latency shifts in the N2b component track phonological deviations in spoken words. Clinical Neurophysiology, 111(1), 4044. https://doi.org/10.1016/s1388-2457(99)00210-2

Dink, J. W., \& Ferguson, B. (2015). EyetrackingR: An R Library for Eye-tracking Data Analysis. http://www.eyetrackingr.com

Dye, M. W. G., \& Shih, S.-I. (2006). Phonological priming in British Sign Language. In Lois Goldstein, D. H. Whalen, \& C. T. Best (Eds.), Laboratory Phonology (Vol. 8, pp. 243-263). De Gruyter Mouton.

Ebbinghaus, H., \& Heßmann, J. (2001). Sign language as multidimensional communication: Why manual signs, mouthings, and mouth gestures are three different things. In P. Boyes Braem \& R. Sutton-Spence (Eds.), The hands are the head of the mouth (pp. 133-151). Signum Verlag.

Eichmann, H., \& Rosenstock, R. (2014). Regional variation in German Sign Language: The role of schools (re-)visited. Sign Language Studies, 14(2), 175-202. https://doi.org/10.1353/sls.2014.0001

Emmorey, K. (1997). Non-antecedent suppression in American Sign Language. Language and Cognitive Processes, 12(1), 103-120. https://doi.org/10.1080/016909697386925 
Emmorey, K. (2002). Language, Cognition, and the Brain: Insights From Sign Language Research. Lawrence Erlbaum and Associates.

Emmorey, K. (2003). The neural systems underlying sign language. In M. Marschark \& P. E. Spencer (Eds.), Oxford handbook of deaf studies, language, and education (pp. 361378). Oxford University Press.

https://books.google.de/books?hl=de\&lr=\&id=8PhwXf9uj1IC\&oi=fnd\&pg=PA361\&d $\mathrm{q}=$ The + Neural + Systems + Underlying + Sign + Language \&ots $=$ ZANMaa2CBC\&sig $=$ Oe AR1dMaNz5Nakg8ed2g23PpkVs

Emmorey, K. (2007). The psycholinguistics of signed and spoken languages: How biology affects processing. In G. Gaskell (Ed.), The Oxford Handbook of Psycholinguistics (pp. 703-721). Oxford University Press.

Emmorey, K., \& Corina, D. P. (1990). Lexical Recognition in Sign Language: Effects of Phonetic Structure and Morphology. Perceptual and Motor Skills, 71(3), 1227-1252. https://doi.org/10.2466/pms.1990.71.3f.1227

Emmorey, K., Corina, D. P., \& Bellugi, U. (1995). Differential processing of topographic and referential functions of space. In K. Emmorey \& J. S. Reilly (Eds.), Language, gesture and space. Lawrence Erlbaum and Associates.

Emmorey, K., \& Falgier, B. (2004). Conceptual locations and pronominal reference in American Sign Language. Journal of Psycholinguistic Research, 33(4), 321-331. https://doi.org/10.1023/b:jopr.0000035104.83502.0b

Emmorey, K., \& Lillo-Martin, D. (1995). Processing spatial anaphora: Referent reactivation with overt and null pronouns in American Sign Language. Language and Cognitive Processes, 10(6), 631-653. https://doi.org/10.1080/01690969508407116

Emmorey, K., McCullough, S., Mehta, S., \& Grabowski, T. J. (2014). How sensory-motor systems impact the neural organization for language: Direct contrasts between spoken and signed language. Frontiers in Psychology, 5. https://doi.org/10.3389/fpsyg.2014.00484

Emmorey, K., McCullough, S., Mehta, S., Ponto, L. L., \& Grabowski, T. J. (2013). The biology of linguistic expression impacts neural correlates for spatial language. Journal of Cognitive Neuroscience, 25(4), 517-533.

Emmorey, K., Norman, F., \& O'Grady, L. (1991). The activation of spatial antecedents from overt pronouns in American Sign Language. Language and Cognitive Processes, 6(3), 207-228. https://doi.org/10.1080/01690969108406943

Emmorey, K., Thompson, R. L., \& Colvin, R. (2009). Eye Gaze During Comprehension of 
American Sign Language by Native and Beginning Signers. Journal of Deaf Studies and Deaf Education, 14(2), 237-243. https://doi.org/10.1093/deafed/enn037

Engberg-Pedersen, E. (1993). Space in Danish Sign Language: The Semantics and Morphosyntax of the Use of Space in a Visual Language. Signum Verlag.

Federmeier, K. D., \& Kutas, M. (1999). Right words and left words: Electrophysiological evidence for hemispheric differences in meaning processing. Cognitive Brain Research, 8(3), 373-392. https://doi.org/10.1016/s0926-6410(99)00036-1

Fischer, S. D. (1975). Influences on Word Order Change in American Sign Language. In C. N. Li (Ed.), Word Order and Word Order Change (pp. 1-25). University of Texas Press.

Flecha-García, M. L. (2010). Eyebrow raises in dialogue and their relation to discourse structure, utterance function and pitch accents in English. Speech Communication, 52(6), 542-554. https://doi.org/10.1016/j.specom.2009.12.003

Foraker, S., \& McElree, B. (2007). The role of prominence in pronoun resolution: Active versus passive representations. Journal of Memory and Language, 56(3), 357-383. https://doi.org/10.1016/j.jml.2006.07.004

Frazier, L., \& d'Arcais, G. B. F. (1989). Filler driven parsing: A study of gap filling in Dutch. Journal of Memory and Language, 28(3), 331-344. https://doi.org/10.1016/0749596x(89)90037-5

Frederiksen, A. T. (2019). Referential Cohesion in American Sign Language: ModalitySpecific and Modality-General Influences. UC San Diego.

Frederiksen, A. T., \& Mayberry, R. I. (2016). Who's on First? Investigating the referential hierarchy in simple native ASL narratives. Lingua, 180, 49-68. https://doi.org/10.1016/j.lingua.2016.03.007

Frederiksen, A. T., \& Mayberry, R. I. (2017, February 20). Pronominalizing Subjects: Referentially Empty Pronouns in ASL? Workshop on Anaphora Resolution on Sign and Spoken Languages - Theoretical and Experimental Dimensions, Göttingen, Germany.

Friederici, A. D., \& Jacobsen, T. (1999). Processing grammatical gender during language comprehension. Journal of Psycholinguistic Research, 28(5), 467-484. https://doi.org/10.1023/A:1023264209610

Friedman, L. A. (1975). Space, time, and person reference in American Sign Language. Language, 940-961.

Geraci, C. (2013, June 19). Spatial syntax in your hands. Linguistic Seminar, CNRS Institut 
Jean-Nicod, Paris.

Geraci, C. (2014). Spatial syntax in your hands. In J. Iyer \& L. Kusmer (Eds.), Proceedings of the Forty-Fourth Annual Meeting of the North East Linguistic Society (pp. 123-134). GLSA. https://sites.google.com/site/carlogeraci76/home

Gernsbacher, M. A. (1989). Mechanisms that improve referential access. Cognition, 32(2), 99-156. https://doi.org/10.1016/0010-0277(89)90001-2

Gernsbacher, M. A., \& Hargreaves, D. J. (1988). Accessing sentence participants: The advantage of first mention. Journal of Memory and Language, 27(6), 699-717. https://doi.org/10.1016/0749-596X(88)90016-2

Gernsbacher, M. A., Hargreaves, D. J., \& Beeman, M. (1989). Building and accessing clausal representations: The advantage of first mention versus the advantage of clause recency. Journal of Memory and Language, 28(6), 735-755. https://doi.org/10.1016/0749-596x(89)90006-5

Gernsbacher, M. A., \& Jescheniak, J. D. (1995). Cataphoric devices in spoken discourse. Cognitive Psychology, 29(1), 24-58.

Givón, T. (1976). Topic, Pronoun and Grammatical Agreement in Subject and Topic. (C. N. Li, Ed.; pp. 149-188). Academic Press.

Gordon, P. C., Grosz, B. J., \& Gilliom, L. A. (1993). Pronouns, names, and the centering of attention in discourse. Cognitive Science, 17(3), 311-347. https://doi.org/10.1207/s15516709cog1703_1

Greene, S. B., McKoon, G., \& Ratcliff, R. (1992). Pronoun resolution and discourse models. Journal of Experimental Psychology: Learning, Memory, and Cognition, 18(2), 266283. https://doi.org/10.1037//0278-7393.18.2.266

Greenhouse, S. W., \& Geisser, S. (1959). On methods in the analysis of profile data. Psychometrika, 24(2), 95-112. https://doi.org/10.1007/bf02289823

Grosjean, F. (1980). Sign and Word Recognition: A First Comparison. Sign Language Studies, 32, 195-220. https://doi.org/10.1353/sls.1982.0003

Grosvald, M., Gutierrez, E., Hafer, S., \& Corina, D. P. (2012). Dissociating linguistic and non-linguistic gesture processing: Electrophysiological evidence from American Sign Language. Brain and Language, 121(1), 12-24. https://doi.org/10.1016/j.bandl.2012.01.005

Gundel, J. K., Hedberg, N., \& Zacharski, R. (1993). Cognitive status and the form of referring expressions in discourse. Language, 274-307. https://doi.org/10.2307/416535

Gutiérrez, E., Müller, O., Baus, C., \& Carreiras, M. (2012). Electrophysiological evidence for 
phonological priming in Spanish Sign Language lexical access. Neuropsychologia, 50(7), 1335-1346. https://doi.org/10.1016/j.neuropsychologia.2012.02.018

Gutiérrez, E., Williams, D., Grosvald, M., \& Corina, D. P. (2012). Lexical access in American Sign Language: An ERP investigation of effects of semantics and phonology. Brain Research, 1468, 63-83. https://doi.org/10.1016/j.brainres.2012.04.029

Hagoort, P., Brown, C., \& Groothusen, J. (1993). The syntactic positive shift (SPS) as an ERP measure of syntactic processing. Language and Cognitive Processes, 8(4), 439-483. https://doi.org/10.1080/01690969308407585

Hänel-Faulhaber, B., Skotara, N., Kügow, M., Salden, U., Bottari, D., \& Röder, B. (2014). ERP correlates of German Sign Language processing in deaf native signers. BMC Neuroscience, 15(1), 62. https://doi.org/10.1186/1471-2202-15-62

Hanna, J. E., \& Tanenhaus, M. K. (2004). Pragmatic effects on reference resolution in a collaborative task: Evidence from eye movements. Cognitive Science, 28(1), 105-115. https://doi.org/10.1207/s15516709cog2801_5

Happ, D., \& Vorköper, M.-O. (2006). Deutsche Gebärdensprache: Ein Lehr-und Arbeitsbuch. Fachhochschulverlag.

Haupt, F. S., Schlesewsky, M., Roehm, D., Friederici, A. D., \& Bornkessel-Schlesewsky, I. (2008). The status of subject-object reanalyses in the language comprehension architecture. Journal of Memory and Language, 59(1), 54-96. https://doi.org/10.1016/j.jml.2008.02.003

Herrmann, A. (2014). Modal and Focus Particles in Sign Languages: A Cross-Linguistic Study. De Gruyter Mouton.

Herrmann, A., \& Steinbach, M. (2007). Wenn ‘ich'nicht ich ist: Zitieren in Gebärdensprachen. In E. Brendel, J. Meibauer, \& M. Steinbach (Eds.), Zitat und Bedeutung (Vol. 15, pp. 153-179). Buske.

Hickok, G., Wilson, M., Clark, K., Klima, E. S., Kritchevsky, M., \& Bellugi, U. (1999). Discourse deficits following right hemisphere damage in deaf signers. Brain and Language, 66(2), 233-248. https://doi.org/10.1006/brln.1998.1995

Hildebrandt, U., \& Corina, D. P. (2002). Phonological similarity in American Sign Language. Language and Cognitive Processes, 17(6), 593-612. https://doi.org/10.1080/01690960143000371

Hillinger, M. L. (1980). Priming effects with phonemically similar words. Memory \& Cognition, 8(2), 115-123. https://doi.org/10.3758/bf03213414 
Holcomb, P. J., Coffey, S. A., \& Neville, H. J. (1992). Visual and auditory sentence processing: A developmental analysis using event-related brain potentials. Developmental Neuropsychology, 8(2 \& 3), 203-241. https://doi.org/10.1080/87565649209540525

Hosemann, J. (2011). Eye gaze and verb agreement in German Sign Language: A first glance. Sign Language \& Linguistics, 14(1), 76-93. https://doi.org/10.1075/s1l.14.1.05hos Hosemann, J. (2015). The processing of German Sign Language sentences. Event-related Potential Studies on Phonological, Morphosyntactic, and Semantic Aspects. [Doctoral dissertation, University of Goettingen]. https://ediss.uni-goettingen.de

Hosemann, J., Herrmann, A., Sennhenn-Reulen, H., Schlesewsky, M., \& Steinbach, M. (2018). Agreement or no agreement. ERP correlates of verb agreement violation in German Sign Language. Language, Cognition and Neuroscience, 33(9), 1107-1127. https://doi.org/10.1080/23273798.2018.1465986

Hosemann, J., Herrmann, A., Steinbach, M., Bornkessel-Schlesewsky, I., \& Schlesewsky, M. (2013). Lexical prediction via forward models: N400 evidence from German Sign Language. Neuropsychologia, 51(11), 2224-2237. https://doi.org/10.1016/j.neuropsychologia.2013.07.013

Hosemann, J., Mani, N., Herrmann, A., Steinbach, M., \& Altvater-Mackensen, N. (2020). Signs activate their written word translation in deaf adults: An ERP study on crossmodal co-activation in German Sign Language. Glossa: A Journal of General Linguistics, 5(1), 57. https://doi.org/10.5334/gjgl.1014

Huettig, F., Rommers, J., \& Meyer, A. S. (2011). Using the visual world paradigm to study language processing: A review and critical evaluation. Acta Psychologica, 137(2), 151-171. https://doi.org/10.1016/j.actpsy.2010.11.003

Jantunen, T. (2010, September). On the role of transitions in signed languages. Theoretical Issues in Sign Language Research (TISLR 10), Purdue University, Indiana, USA. http://www.purdue.edu/tislr10/pdfs/Jantunnen\%20transitions.pdf

Järvikivi, J., van Gompel, R. P., Hyönä, J., \& Bertram, R. (2005). Ambiguous Pronoun Resolution: Contrasting the First-Mention and Subject-Preference Accounts. Psychological Science, 16(4), 260-264. https://doi.org/10.1111/j.09567976.2005.01525.x

Just, M. A., \& Carpenter, P. A. (1980). A theory of reading: From eye fixations to comprehension. Psychological Review, 87(4), 329-354. https://doi.org/10.1037//0033$295 x .87 .4 .329$ 
Kaan, E., Harris, A., Gibson, E., \& Holcomb, P. (2000). The P600 as an index of syntactic integration difficulty. Language and Cognitive Processes, 15(2), 159-201. https://doi.org/10.1080/016909600386084

Kaiser, E. (2011). Focusing on pronouns: Consequences of subjecthood, pronominalisation, and contrastive focus. Language and Cognitive Processes, 26(10), 1625-1666. https://doi.org/10.1080/01690965.2010.523082

Kaiser, E. (2006). Effects of topic and focus on salience. In C. Ebert \& C. Endriss (Eds.), Proceedings of Sinn und Bedeutung (Vol. 10, pp. 139-154).

Kamp, H., \& Reyle, U. (1993). From discourse to logic: Introduction to modeltheoretic semantics of natural language, formal logic and discourse representation theory. Kluwer Academic Publishers.

Kenstowicz, M., \& Kisseberth, C. (1979). Generative phonology: Description and theory. Academic Press.

Kimura, M., Schröger, E., \& Czigler, I. (2011). Visual mismatch negativity and its importance in visual cognitive sciences. Neuroreport, 22(14), 669-673. https://doi.org/10.1097/wnr.0b013e32834973ba

Klima, E. S., \& Bellugi, U. (1979). The Signs of Language. Harvard University Press.

Krebs, J. (2013). The processing of word order variations in Austrian Sign Language (ÖGS). An ERP-study on subject preference. [Master's Thesis]. Universität Salzburg.

Krebs, J. (2017). The Syntax and the Processing of Argument Relations in Austrian Sign Language $(\ddot{O} G S)$ [Doctoral dissertation]. Universität Salzburg.

Krebs, J., Malaia, E., Wilbur, R. B., \& Roehm, D. (2018). Subject preference emerges as cross-modal strategy for linguistic processing. Brain Research, 1691, 105-117. https://doi.org/10.1016/j.brainres.2018.03.029

Krebs, J., Malaia, E., Wilbur, R. B., \& Roehm, D. (2019). Interaction between topic marking and subject preference strategy in sign language processing. Language, Cognition and Neuroscience, 1-19. https://doi.org/10.1080/23273798.2019.1667001

Krebs, J., Wilbur, R. B., Alday, P. M., \& Roehm, D. (2019). The impact of transitional movements and non-manual markings on the disambiguation of locally ambiguous argument structures in Austrian Sign Language (ÖGS). Language and Speech, 62(4), $652-680$.

Kubus, O., Villwock, A., Morford, J. P., \& Rathmann, C. (2015). Word recognition in deaf readers: Cross-language activation of German Sign Language and German. Applied Psycholinguistics, 36(4), 831-854. https://doi.org/10.1017/S0142716413000520 
Kutas, M., \& Federmeier, K. D. (2000). Electrophysiology reveals semantic memory use in language comprehension. Trends in Cognitive Sciences, 4(12), 463-470. https://doi.org/10.1016/s1364-6613(00)01560-6

Kutas, M., \& Federmeier, K. D. (2011). Thirty years and counting: Finding meaning in the N400 component of the event-related brain potential (ERP). Annual Review of Psychology, 62, 621-647. https://doi.org/10.1146/annurev.psych.093008.131123

Kutas, M., Neville, H. J., \& Holcomb, P. J. (1987). A preliminary comparison of the N400 response to semantic anomalies during reading, listening and signing. Electroencephalography and Clinical Neurophysiology Supplement, 325-330.

Langer, G. (2012). A colorful first glance at data on regional variation extracted from the dgscorpus: With a focus on procedures. 5th Workshop on the Representation and Processing of Sign Languages: Interactions between Corpus and Lexicon, 101-108. https://www.sign-lang.uni-hamburg.de/dgskorpus/files/inhalt_pdf/LREC_2012_Colours.pdf

Lau, E. F., Phillips, C., \& Poeppel, D. (2008). A cortical network for semantics: (De)constructing the N400. Nature Reviews Neuroscience, 9(12), 920-933. https://doi.org/10.1038/nrn2532

Leeson, L., \& Saeed, J. (2012). Word order. In R. Pfau, M. Steinbach, \& B. Woll (Eds.), Sign Language: An International Handbook (pp. 245-265). De Gruyter.

Lenneberg, E. H. (1967). The biological foundations of language. Wiley.

Leonard, M. K., Ramirez, N. F., Torres, C., Travis, K. E., Hatrak, M., Mayberry, R. I., \& Halgren, E. (2012). Signed words in the congenitally deaf evoke typical late lexicosemantic responses with no early visual responses in left superior temporal cortex. The Journal of Neuroscience, 32(28), 9700-9705.

Levänen, S., Uutela, K., Salenius, S., \& Hari, R. (2001). Cortical representation of sign language: Comparison of deaf signers and hearing non-signers. Cerebral Cortex, $11(6), 506-512$.

Li, C. N., \& Thompson, S. L. (1976). Subject and topic: A new typology of language. In C. N. Li (Ed.), Subject and topic (pp. 459-489). Academic Press.

Liddell, S. K. (1980). American sign language syntax (Vol. 52). Mouton De Gruyter.

Liddell, S. K. (1984). Think and Belive: Sequentiality in American Sign Language. Language, 60(2), 372-399. https://doi.org/10.2307/413645

Liddell, S. K. (1990). Four functions of a locus: Re-examining the structure of space in ASL. In C. Lucas (Ed.), Sign language research: Theoretical issues (pp. 176-198). 
Gallaudet University Press.

Liddell, S. K. (1995). Real, Surrogate, and Token Space: Grammatical Consequences in ASL.

In K. Emmorey \& J. S. Reilly (Eds.), Language, Gesture, and Space (pp. 19-42).

Lawrence Erlbaum Associates.

Liddell, S. K., \& Johnson, R. E. (1989). American Sign Language: The phonological base.

Sign Language Studies, 64(1), 195-277. https://doi.org/10.1353/sls.1989.0027

Lieberman, A. M., Borovsky, A., Hatrak, M., \& Mayberry, R. I. (2015). Real-time Processing of ASL signs: Delayed First Language Acquisition Affects Organization of the Mental Lexicon. Journal of Experimental Psychology: Learning, Memory, and Cognition, 41(4), 1130-1139. https://doi.org/10.1037/xlm0000088

Lieberman, A. M., Borovsky, A., \& Mayberry, R. I. (2017). Prediction in a visual language: Real-time sentence processing in American Sign Language across development. Language, Cognition and Neuroscience, 1-15. https://doi.org/10.1080/23273798.2017.1411961

Lillo-Martin, D. (1986). Two kinds of null arguments in American Sign Language. Natural Language \& Linguistic Theory, 4(4), 415-444. https://doi.org/10.1007/bf00134469

Lillo-Martin, D. (2012). Utterance reports and constructed action in sign and spoken languages. In R. Pfau, M. Steinbach, \& B. Woll (Eds.), Sign Language: An International Handbook (pp. 365-387). De Gruyter Mouton.

Lillo-Martin, D., \& de Quadros, R. M. (2008). Focus constructions in American Sign Language and Língua de Sinais Brasileira. In J. Quer (Ed.), Signs of the time: Selected papers from TISLR 8 (pp. 161-176). Signum.

Lillo-Martin, D., \& Klima, E. S. (1990). Pointing Out Differences: ASL Pronouns in Syntactic Theory. In S. D. Fischer \& P. Siple (Eds.), Theoretical Issues in Sign Language Research, Volume 1: Linguistics. University of Chicago Press.

Lillo-Martin, D., \& Meier, R. P. (2011). On the linguistic status of 'agreement' in sign languages. Theoretical Linguistics, 37(3-4), 95-141.

Loew, R. C., Kegl, J. A., \& Poizner, H. (1997). Fractionation of the components of role play in a right-hemispheric lesioned signer. Aphasiology, 11(3), 263-281. https://doi.org/10.1080/02687039708248469

Loke, W. H., \& Song, S. (1991). Central and peripheral visual processing in hearing and nonhearing individuals. Bulletin of the Psychonomic Society, 29(5), 437-440.

Lopez-Calderon, J., \& Luck, S. J. (2014). ERPLAB: An open-source toolbox for the analysis of event-related potentials. Frontiers in Human Neuroscience, 8(213), 1-14. 
https://doi.org/10.3389/fnhum.2014.00213

Luck, S. J. (2005). An introduction to the event-related brain potential technique. MIT Press.

Macht, C., \& Steinbach, M. (2019). Regionalsprachliche Merkmale in der Deutschen Gebärdensprache. In J. Herrgen \& J. E. Schmidt (Eds.), Sprache und Raum. Ein internationales Handbuch der Sprachvariation (pp. 916-937). De Gruyter Mouton.

MacLaughlin, D. (1997). The Structure of Determiner Phrases: Evidence from ASL [Doctoral dissertation]. MIT.

MacSweeney, M., Capek, C. M., Campbell, R., \& Woll, B. (2008). The signing brain: The neurobiology of sign language. Trends in Cognitive Sciences, 12(11), 432-440. https://doi.org/10.1016/j.tics.2008.07.010

MacSweeney, M., Waters, D., Brammer, M. J., Woll, B., \& Goswami, U. (2008). Phonological processing in deaf signers and the impact of age of first language acquisition. NeuroImage, 40(3), 1369-1379. https://doi.org/10.1016/j.neuroimage.2007.12.047

MacSweeney, M., Woll, B., Campbell, R., McGuire, P. K., David, A. S., Williams, S. C. R., Suckling, J., Calvert, G. A., \& Brammer, M. J. (2002). Neural systems underlying British Sign Language and audio-visual English processing in native users. Brain, 125(7), 1583-1593. https://doi.org/10.1093/brain/awf153

Marslen-Wilson, W. D., \& Welsh, A. (1978). Processing interactions and lexical access during word recognition in continuous speech. Cognitive Psychology, 10(1), 29-63. https://doi.org/10.1016/0010-0285(78)90018-X

Mathur, G., \& Rathmann, C. (2012). Verb agreement. In R. Pfau, M. Steinbach, \& B. Woll (Eds.), Sign Language: An International Handbook. De Gruyter Mouton.

Mayberry, R. I. (1993). First-language acquisition after childhood differs from secondlanguage acquisition: The case of American Sign Language. Journal of Speech, Language, and Hearing Research, 36(6), 1258-1270.

Mayberry, R. I., \& Witcher, P. (2005). What Age of Acquisition Effects Reveal about the Nature of Phonological Processing. Center for Research in Language Technical Reports, 17(3), 3-9.

McBurney, S. L. (2002). Pronominal reference in signed and spoken language: Are grammatical categories modality-dependent? In R. P. Meier, K. Cormier, \& D. Quinto-Pozos (Eds.), Modality and Structure in Signed and Spoken Language (pp. 329-369). Cambridge University Press.

McClelland, J. L., \& Rumelhart, D. E. (1981). An interactive activation model of context 
effects in letter perception: Part I. An account of basic findings. Psychological Review, 88(5), 375. https://doi.org/10.1016/b978-1-4832-1446-7.50048-0

McKee, R., Schembri, A., McKee, D., \& Johnston, T. (2011). Variable "subject" presence in Australian Sign Language and New Zealand Sign Language. Language Variation and Change, 23(3), 375-398. https://doi.org/10.1017/S0954394511000123

McKoon, G., Gerrig, R. J., \& Greene, S. B. (1996). Pronoun resolution without pronouns: Some consequences of memory-based text processing. Journal of Experimental Psychology: Learning, Memory, and Cognition, 22(4), 919.

McLaughlin, J., Osterhout, L., \& Kim, A. (2004). Neural correlates of second-language word learning: Minimal instruction produces rapid change. Nature Neuroscience, 7(7), 703704. https://doi.org/10.1038/nn1264

Meade, G., Lee, B., Midgley, K. J., Holcomb, P. J., \& Emmorey, K. (2018). Phonological and semantic priming in American Sign Language: N300 and N400 effects. Language, Cognition and Neuroscience, 33(9), 1092-1106. https://doi.org/10.1080/23273798.2018.1446543

Meade, G., Midgley, K. J., Sevcikova Sehyr, Z., Holcomb, P. J., \& Emmorey, K. (2017). Implicit co-activation of American Sign Language in deaf readers: An ERP study. Brain and Language, 170, 50-61. https://doi.org/10.1016/j.bandl.2017.03.004

Meier, R. P. (1990). Person deixis in American Sign Language. Theoretical Issues in Sign Language Research, 1, 175-190.

Meier, R. P. (2002). Why different, why the same? Explaining effects and non-effects of modality upon linguistic structure in sign and speech. In R. P. Meier, K. Cormier, \& D. Quinto-Pozos (Eds.), Modality and Structure in Signed and Spoken Languages (pp. 1-25). Cambridge University Press.

Meier, R. P. (2012). Language and Modality. In R. Pfau, M. Steinbach, \& B. Woll (Eds.), Sign Language: An International Handbook (pp. 574-601). De Gruyter Mouton.

Meir, I. (2002). A cross-modality perspective on verb agreement. Natural Language \& Linguistic Theory, 20(2), 413-450. https://doi.org/10.1023/A:1015041113514

Meir, I., \& Sandler, W. (2008). A language in space: The story of Israeli Sign Language. Lawrence Erlbaum Associates.

Melinger, A., \& Weber, A. (2006). Database of Noun Associations for German. http://www.psycholing.es.uni-tuebingen.de/nag/index.php

Midgley, K. J., Holcomb, P. J., \& Grainger, J. (2009). Language effects in second language learners and proficient bilinguals investigated with event-related potentials. Journal of 
Neurolinguistics, 22(3), 281-300. https://doi.org/10.1016/j.jneuroling.2008.08.001 Morford, J. P., Wilkinson, E., Villwock, A., Piñar, P., \& Kroll, J. F. (2011). When deaf signers read English: Do written words activate their sign translations? Cognition, 118(2), 286-292. https://doi.org/10.1016/j.cognition.2010.11.006

Morgan, G., Barrett-Jones, S., \& Stoneham, H. (2007). The first signs of language: Phonological development in British Sign Language. Applied Psycholinguistics, 28(01), 3-22. https://doi.org/10.1017/S0142716407070014

Muir, L. J., \& Richardson, I. E. (2005). Perception of sign language and its application to visual communications for deaf people. Journal of Deaf Studies and Deaf Education, 10(4), 390-401.

Münte, T. F., Heinze, H.-J., Matzke, M., Wieringa, B. M., \& Johannes, S. (1998). Brain potentials and syntactic violations revisited: No evidence for specificity of the syntactic positive shift. Neuropsychologia, 36(3), 217-226. https://doi.org/10.1016/s0028-3932(97)00119-x

Münte, T. F., Matzke, M., \& Johannes, S. (1997). Brain activity associated with syntactic incongruencies in words and pseudo-words. Journal of Cognitive Neuroscience, 9(3), 318-329. https://doi.org/10.1162/jocn.1997.9.3.318

Murmann, C. (2012). The agreement auxiliary PAM in German Sign Language—An empirical investigation [Master's Thesis]. Heinrich Heine Universität.

Neidle, C. (2002). Language across modalities: ASL focus and question constructions. Linguistic Variation Yearbook, 2(1), 71-98. https://doi.org/10.1075/livy.2.05nei

Neidle, C., Kegl, J., MacLaughlin, D., Bahan, B., \& Lee, R. G. (2000). The syntax of American Sign Language: Functional categories and hierarchical structure. MIT Press.

Neidle, C., \& Nash, J. (2012). The noun phrase. In R. Pfau, M. Steinbach, \& B. Woll (Eds.), Sign Language: An International Handbook (pp. 265-292). De Gruyter Mouton.

Neville, H. J. (1990). Intermodal competition and compensation in development. Annals of the New York Academy of Sciences, 608(1), 71-91.

Neville, H. J., Coffey, S. A., Lawson, D. S., Fischer, A., Emmorey, K., \& Bellugi, U. (1997). Neural Systems Mediating American Sign Language: Effects of Sensory Experience and Age of Acquisition. Brain and Language, 57(3), 285-308. https://doi.org/10.1006/brln.1997.1739

Neville, H. J., \& Lawson, D. (1987). Attention to central and peripheral visual space in a movement detection task. III. Separate effects of auditory deprivation and acquisition 
of a visual language. Brain Research, 405(2), 284-294. https://doi.org/10.1016/00068993(87)90297-6

Newman, R. L., Connolly, J. F., Service, E., \& Mcivor, K. (2003). Influence of phonological expectations during a phoneme deletion task: Evidence from event-related brain potentials. Psychophysiology, 40(4), 640-647. https://doi.org/10.1111/14698986.00065

Newport, E. L. (1990). Maturational constraints on language learning. Cognitive Science, 14(1), 11-28.

Nicol, J., \& Swinney, D. (1989). The role of structure in coreference assignment during sentence comprehension. Journal of Psycholinguistic Research, 18(1), 5-19. https://doi.org/10.1007/bf01069043

Nuhbalaoglu, D. (2018). Comprehension and production of referential expressions in German Sign Language: An empirical approach. [Doctoral dissertation, University of Goettingen]. https://ediss.uni-goettingen.de/handle/21.11130/00-1735-0000 -0003C152-6

Orfanidou, E., Adam, R., McQueen, J. M., \& Morgan, G. (2009). Making sense of nonsense in British Sign Language (BSL): The contribution of different phonological parameters to sign recognition. Memory \& Cognition, 37(3), 302-315. https://doi.org/10.3758/mc.37.3.302

Padden, C. (1990). The relation between space and grammar in ASL verb morphology. In C. Lucas (Ed.), Sign language research: Theoretical issues (pp. 118-132). Gallaudet University Press.

Padden, C., \& Humphries, T. (1990). Deaf in America. Harvard University Press.

Perniss, P. (2012). Use of sign space. In R. Pfau, M. Steinbach, \& B. Woll (Eds.), Sign Language: An International Handbook (pp. 412-431). De Gruyter Mouton.

Perniss, P. M. (2007). Space and iconicity in German Sign Language (DGS) [Doctoral dissertation, Radboud University]. http://repository.ubn.ru.nl/handle/2066/30937

Perniss, P. M., \& Özyürek, A. (2015). Visible Cohesion: A Comparison of Reference Tracking in Sign, Speech, and Co-Speech Gesture. Topics in Cognitive Science, 7(1), 36-60. https://doi.org/10.1111/tops.12122

Pfau, R. (1997). Zur phonologischen Komponente der Deutschen Gebärdensprache: Segmente und Silben. Frankfurter Linguistische Forschungen, 20, 1-29.

Pfau, R. (2011). A point well taken: On the typology and diachrony of pointing. In D. J. Napoli \& G. Mathur (Eds.), Deaf around the world: The impact of language (pp. 144- 
163). Oxford University Press.

Pfau, R., \& Quer, J. (2010). Nonmanuals: Their grammatical and prosodoc roles. In D.

Brentari (Ed.), Sign Languages (Cambridge Language Sruveys) (pp. 381-402).

Cambridge University Press.

Pfau, R., \& Steinbach, M. (2003). Optimal reciprocals in German Sign Language. Sign

Language \& Linguistics, 6(1), 3-42. https://doi.org/10.1075/s1l.6.1.03pfa

Pfau, R., \& Steinbach, M. (2006). Pluralization in sign and in speech: A cross-modal typological study. Linguistic Typology, 10(2), 135-182.

https://doi.org/10.1515/LINGTY.2006.006,

Pfau, R., \& Steinbach, M. (2013). PERSON Climbing up a Tree (and other Adventures in Sign Language Grammaticalization). Sign Language \& Linguistics, 16(2), 189-220. https://doi.org/10.1075/s1l.16.2.04pfa

Poizner, H. (1983). Perception of movement in American Sign Language: Effects of linguistic structure and linguistic experience. Attention, Perception, \& Psychophysics, 33(3), 215-231. https://doi.org/10.3758/bf03202858

Poizner, H., Klima, E. S., \& Bellugi, U. (1987). What the Hands Reveal about the Brain. MIT Press.

Radeau, M., Morais, J., \& Segui, J. (1995). Phonological priming between monosyllabic spoken words. Journal of Experimental Psychology: Human Perception and Performance, 21(6), 1297-1310. https://doi.org/10.1037//0096-1523.21.6.1297

Rathmann, C. (2003). The optionality of agreement phrase: Evidence from German Sign Language (DGS). In W. E. Griffin (Ed.), The role of agreement in natural language: Proceedings of the Fifth Annual Texas Linguistics Society Conference (pp. 181-192). Texas Linguistic Forum.

Reynolds, H. N. (1993). Effects of foveal stimulation on peripheral visual processing and laterality in deaf and hearing subjects. The American Journal of Psychology, 523-540.

Rugg, M. D., Lines, C. R., \& Milner, A. D. (1985). Further investigation of visual evoked potentials elicited by lateralized stimuli: Effects of stimulus eccentricity and reference site. Electroencephalography and Clinical Neurophysiology/Evoked Potentials Section, 62(2), 81-87. https://doi.org/10.1016/0168-5597(85)90019-x

Sandler, W. (1986). The Spreading Hand Autosegment of American Sign Language. Sign Language Studies, 50(1), 1-28. https://doi.org/10.1353/sls.1986.0006

Sandler, W. (1989). Phonological representation of the sign: Linearity and nonlinearity in American Sign Language (Vol. 32). De Gruyter Mouton. 
https://books.google.de/books?hl=de\&lr=\&id=vOTny0iScYwC\&oi=fnd\&pg=PR4\&dq $=$ sandler + sign + language + phonology\&ots $=5$ WIVHXZtQv\&sig $=\mathrm{qAY}$ oxu038E5z3CaT 6U_OJ20tJks

Sandler, W. (2012). Visual prosody. In R. Pfau, M. Steinbach, \& B. Woll (Eds.), Sign Language: An International Handbook (pp. 55-76). De Gruyter Mouton.

Sandler, W., \& Lillo-Martin, D. (2006). Sign Language and Linguistic Universals. Cambridge University Press.

Schlenker, P. (2013). Anaphora: Insights from Sign Language. In S. R. Anderson, J. Moeschler, \& F. Reboul (Eds.), The Language-Cognition Interface: Actes du 10e Congrès International des Linguistes. Librairie Droz.

Schlenker, P., Lamberton, J., \& Santoro, M. (2013). Iconic variables. Linguistics and Philosophy, 36(2), 91-149. https://doi.org/10.1007/s10988-013-9129-1

Schriefers, H., Friederici, A. D., \& Kuhn, K. (1995). The processing of locally ambiguous relative clauses in German. Journal of Memory and Language, 34(4), 499-520. https://doi.org/10.1006/jmla.1995.1023

Schütze, C. T. (2016). The empirical base of linguistics: Grammaticality judgments and linguistic methodology. Language Science Press. http://langscipress.org/catalog/book/89

Siedlecki Jr, T., \& Bonvillian, J. D. (1993). Location, Handshape \& Movement: Young Children's Acquisition of the Formational Aspects of American Sign Language. Sign Language Studies, 78(1), 31-52. https://doi.org/10.1353/sls.1993.0016

Slowiaczek, L. M., \& Hamburger, M. (1992). Prelexical facilitation and lexical interference in auditory word recognition. Journal of Experimental Psychology: Learning, Memory, and Cognition, 18(6), 1239-1250. https://doi.org/10.1037//0278-7393.18.6.1239

Slowiaczek, L. M., McQueen, J. M., Soltano, E. G., \& Lynch, M. (2000). Phonological representations in prelexical speech processing: Evidence from form-based priming. Journal of Memory and Language, 43(3), 530-560. https://doi.org/10.1006/jmla.2000.2710

Slowiaczek, L. M., \& Pisoni, D. B. (1986). Effects of phonological similarity on priming in auditory lexical decision. Memory \& Cognition, 14(3), 230-237. https://doi.org/10.3758/bf03197698

Srinivasan, R. J., \& Massaro, D. W. (2003). Perceiving prosody from the face and voice: Distinguishing statements from echoic questions in English. Language and Speech, 46(1), 1-22. https://doi.org/10.1177/00238309030460010201 
St George, M., Kutas, M., Martinez, A., \& Sereno, M. I. (1999). Semantic integration in reading: Engagement of the right hemisphere during discourse processing. Brain, 122(7), 1317-1325. https://doi.org/10.1093/brain/122.7.1317

Steinbach, M., \& Onea, E. (2016). A DRT Analysis of Discourse Referents and Anaphora Resolution in Sign Language. Journal of Semantics, 33(3), 409-448. https://doi.org/10.1093/jos/ffv002

Stivalet, P., Moreno, Y., Richard, J., Barraud, P.-A., \& Raphel, C. (1998). Differences in visual search tasks between congenitally deaf and normally hearing adults. Cognitive Brain Research, 6(3), 227-232.

Stokoe, W. C. (1960). Sign Language Structure: An Outline of the Visual Communication Systems of the American Deaf. University of Buffalo Press.

Stokoe, W. C., Casterline, D. C., \& Croneberg, C. G. (1965). A dictionary of American Sign Language on linguistic principles. Gallaudet College Press.

Sutton-Spence, R., \& Woll, B. (1999). The linguistics of British Sign Language: An Introduction. Cambridge University Press.

Swerts, M., \& Krahmer, E. (2008). Facial expression and prosodic prominence: Effects of modality and facial area. Journal of Phonetics, 36(2), 219-238. https://doi.org/10.1016/j.wocn.2007.05.001

Tanenhaus, M. K., Spivey-Knowlton, M. J., Eberhard, K. M., \& Sedivy, J. C. (1995). Integration of visual and linguistic information in spoken language comprehension. Science, 268(5217), 1632-1634. https://doi.org/10.1126/science.7777863

ten Holt, G. A., Van Doorn, A. J., de Ridder, H., Reinders, M. J. T., \& Hendriks, E. A. (2009). Which fragments of a sign enable its recognition? Sign Language Studies, 9(2), 211-239.

Thierry, G., \& Wu, Y. J. (2007). Brain potentials reveal unconscious translation during foreign-language comprehension. Proceedings of the National Academy of Sciences, 104(30), 12530-12535. https://doi.org/10.1073/pnas.0609927104

Thompson, R. L., Emmorey, K., \& Gollan, T. H. (2005). “Tip of the fingers” Experiences by Deaf Signers. Insights Into the Organization of a Sign-Based Lexicon. Psychological Science, 16(11), 856-860. https://doi.org/10.1111/j.1467-9280.2005.01626.x

Thompson, R. L., Emmorey, K., \& Kluender, R. (2006). The Relationship between Eye Gaze and Verb Agreement in American Sign Language: An Eye-tracking Study. Natural Language \& Linguistic Theory, 24(2), 571-604. https://doi.org/10.1007/s11049-0051829-y 
Thompson, R. L., Emmorey, K., \& Kluender, R. (2009). Learning to look: The acquisition of eye gaze agreement during the production of ASL verbs. Bilingualism: Language and Cognition, 12(4), 393-409.

Thompson, R. L., Vinson, D., Fox, N., \& Vigliocco, G. (2013). Is Lexical Access Driven by Temporal Order or Perceptual Salience? Evidence from British Sign Language. Proceedings of the 35th Annual Meeting of the Cognitive Science Society, 35(35), $1450-1455$.

Twilhaar, J. N., \& van den Bogaerde, B. (2016). Concise Lexicon for Sign Linguistics. John Benjamins Publishing Company.

van Berkum, J. J. A., Brown, C. M., \& Hagoort, P. (1999). Early Referential Context Effects in Sentence Processing: Evidence from Event-Related Brain Potentials. Journal of Memory and Language, 41(2), 147-182. https://doi.org/10.1006/jmla.1999.2641

van Berkum, J. J. A., Koornneef, A. W., Otten, M., \& Nieuwland, M. S. (2007). Establishing reference in language comprehension: An electrophysiological perspective. Brain Research, 1146, 158-171. https://doi.org/10.1016/j.brainres.2006.06.091

van de Meerendonk, N., Indefrey, P., Chwilla, D. J., \& Kolk, H. H. (2011). Monitoring in language perception: Electrophysiological and hemodynamic responses to spelling violations. Neuroimage, 54(3), 2350-2363.

https://doi.org/10.1016/j.neuroimage.2010.10.022

van de Meerendonk, N., Kolk, H. H., Chwilla, D. J., \& Vissers, C. T. W. (2009). Monitoring in language perception. Language and Linguistics Compass, 3(5), 1211-1224. https://doi.org/10.1111/j.1749-818X.2009.00163.x

van der Kooij, E., Crasborn, O., \& Emmerik, W. (2006). Explaining prosodic body leans in Sign Language of the Netherlands: Pragmatics required. Journal of Pragmatics, 38(10), 1598-1614. https://doi.org/0.1016/j.pragma.2005.07.006

van Herten, M., Chwilla, D. J., \& Kolk, H. H. (2006). When heuristics clash with parsing routines: ERP evidence for conflict monitoring in sentence perception. Journal of Cognitive Neuroscience, 18(7), 1181-1197. https://doi.org/10.1162/jocn.2006.18.7.1181

Vissers, C. T. W., Chwilla, D. J., \& Kolk, H. H. (2006). Monitoring in language perception: The effect of misspellings of words in highly constrained sentences. Brain Research, 1106(1), 150-163. https://doi.org/10.1016/j.brainres.2006.05.012

Von Eckardt, B., \& Potter, M. C. (1985). Clauses and the semantic representation of words. Memory \& Cognition, 13(4), 371-376. https://doi.org/10.3758/bf03202505 
Wienholz, A., Nuhbalaoglu, D., Mani, N., Herrmann, A., Onea, E., \& Steinbach, M. (2018). Pointing to the right side? An ERP study on anaphora resolution in German Sign Language. PLoS ONE, 13(9), e0204223. https://doi.org/10.1371/journal.pone.0204223

Wienholz, A., Nuhbalaoglu, D., Mani, N., Herrmann, A., Onea, E., \& Steinbach, M. (submitted). Processing pronominal pointing signs in German Sign Language: Neurophysiological evidence for the first mention effect.

Wienholz, A., Nuhbalaoglu, D., Steinbach, M., Herrmann, A., \& Mani, N. (2018). The influence of overt localization on the processing of referential expressions in German Sign Language [Manuscript in preparation].

Wienholz, A., Nuhbalaoglu, D., Steinbach, M., Herrmann, A., \& Mani, N. (2021). Phonological priming in German Sign Language: An eye tracking study using the Visual World Paradigm. Sign Language \& Linguistics, 24(1), 4-35. https://doi.org/10.1075/sll.19011.wie

Wilbur, R. B. (1997). A prosodic/pragmatic explanation for word order variation in ASL with typological implications. In K. D. Lee, E. Sweetser, \& M. H. Verspoor (Eds.), Lexical and syntactic constructions and the construction of meaning (pp. 89-104). John Benjamins Publishing Company.

Wilbur, R. B. (1999). Stress in ASL: Empirical Evidence and Linguistic Issues. Language and Speech, 42(2-3), 229-250. https://doi.org/10.1177/00238309990420020501

Wilbur, R. B. (2012). Information structure. In R. Pfau, M. Steinbach, \& B. Woll (Eds.), Sign Language: An International Handbook (pp. 462-488). De Gruyter Mouton.

Wilbur, R. B., \& Patschke, C. (1999). Syntactic correlates of brow raise in ASL. Sign Language \& Linguistics, 2(1), 3-41. https://doi.org/10.1075/sll.2.1.03wil

Wilbur, R. B., \& Patschke, C. G. (1998). Body leans and the marking of contrast in American Sign Language. Journal of Pragmatics, 30(3), 275-303. https://doi.org/10.1016/s0378-2166(98)00003-4

Winston, E. A. (1996). Spatial mapping in ASL discourse. In D. M. Jones (Ed.), Assessing Our Work: Assessing Our Worth (pp. 1-28). CIT Conference Proceedings.

Wittenburg, P., Brugman, H., Russel, A., Klassmann, A., \& Sloetjes, H. (2006). ELAN: A professional framework for multimodality research. Proceedings of LREC, 2006.

Woodward, J. C. (1972). Implications for sociolinguistic research among the deaf. Sign Language Studies, 1(1), 1-7.

Wulf, A., Dudis, P., Bayley, R., \& Lucas, C. (2002). Variable Subject Presence in ASL Narratives. Sign Language Studies, 3(1), 54-76. https://doi.org/10.1353/sls.2002.0027 



\section{List of figures}

Figure 1.1 Signing space (A) and its planes (B) 14

Figure 1.2 Structure of a single trial 29

Figure 1.3 Grand average ERPs for congruent (black) and incongruent (red)

conditions. Data are timelocked to the target handshape trigger. C3 and C5 are central right and left lateral electrodes on the left hemisphere. C4 and C6 are central left and right lateral electrodes on the right hemisphere. Negativity is plotted upwards.

Figure 1.4 Grand average ERPs congruent (black) and incongruent (red) conditions.

Data are timelocked to the target handshape trigger for frontal, central and parietal regions across left and right hemisphere. Negativity is plotted upwards.

Figure 1.5 Grand average ERPs for conditions containing an ipsilateral/right (black) and contralateral/left (red) index. Data are timelocked to the target handshape trigger for both left and right hemisphere. Negativity is plotted upwards.

Figure 2.1 Structure of a single trial

Figure 2.2 Grand average ERPs for ipsilateral (black) and contralateral (red) conditions timelocked to the trigger 'direction' for fronto-central, centro-parietal and parietal-occipital regions across left $(\mathrm{LH})$ and right hemisphere $(\mathrm{RH})$.

Negativity is plotted upwards.

Figure 3.1 Structure of a single trial 83 
Figure 3.2 Mean proportion of target looking across time for each continuation type (from top to bottom: object continuation, subject continuation). The conditions are shown as lines (orange $=$ no localization, blue $=$ object localization, green $=$ subject localization, purple $=$ localization both) with 1000 ms previous and 2000 ms following the first fixation to one of the presented pictures.

Figure 3.3 Mean proportion of target looking for object (left panel) and subject (right panel) continuation types and each condition (orange $=$ no localization, blue $=$ object localization, green $=$ subject localization, purple $=$ localization both) for $1000 \mathrm{~ms}$ following the first fixation to one of the presented pictures. .86

Figure 4.1 Overview of prime-target sign pairs with examples from the stimuli: minimal pairs differing in handshape (left column), location (middle column) and movement parameter (right column). In the priming condition, the target (upper row) and the related prime (middle row) are combined. In the unrelated condition, the unrelated prime (bottom row) is combined with the target. 104

Figure 4.2 Structure of a single trial 110

Figure 4.3 Mean proportion of target looking across time for each parameter condition (from top to bottom: location and movement, handshape and movement, handshape and location). The priming (orange) and unrelated (blue) conditions are shown as lines surrounded by shaded areas indicating standard error.

Figure 4.4 Mean proportion of target looking for priming and unrelated conditions for each parameter condition $($ pink $=$ location and movement; green $=$ handshape and movement, yellow $=$ handshape and location) between $1000-3000 \mathrm{~ms}$ following 
target sign onset. Error bars represent standard errors. Asterisks above bars indicate significance levels between bars: $* * p<.01$ 



\section{List of tables}

Table 0.1.1 Overview of selected ERP studies on sign languages 8

Table 0.1.2 Overview of selected eye tracking studies on sign languages. 10

Table 1.1. Mean judgement ratings and standard deviations for each condition. 31

Table 3.1 Results of the best-fitting model for effects of condition, continuation type and their interaction presenting estimates, standard error (SE), t-value and lower $(2,5 \%)$ and upper $(97,5 \%)$ confidence interval (CI). 87

Table 3.2 Results of the models for each condition with fixed effects of continuation type and random effects of participants and items presenting estimates, standard error (SE), t-value and lower (2,5\%) and upper (97,5\%) confidence interval (CI).

Table 4.1 Overview of studies examining effects of phonological parameters 95

Table 4.2 Overview of studies using different methods to investigate phonological priming in sign languages 



\section{Appendix}

Appendix A: Chapter 1 - Scene stills of videos for each condition. XXXII

Appendix B: Chapter $1 \& 2$ - Complete list of stimulus material. XXXIII

Appendix C: Chapter 2 - Schematic illustration of the regions of interest (ROI) ... XLII Appendix D: Chapter 3 - Scene stills of videos for each condition and each continuation type

Appendix E: Chapter 3 - Complete list of stimulus material XLV

Appendix F: Chapter 3 - Figure of the Elog-transformed data . LXI

Appendix G: Chapter 4-Complete list of stimulus material LXII 
Appendix A: Chapter 1 - Scene stills of videos for each condition

Lines of different colors indicate the conditions (orange $=$ Condition 1 , green $=$ Condition 2 , blue $=$ Condition 3 , purple $=$ Condition 4). Please follow lines of the same color to get the sequence of video shown for the corresponding condition.

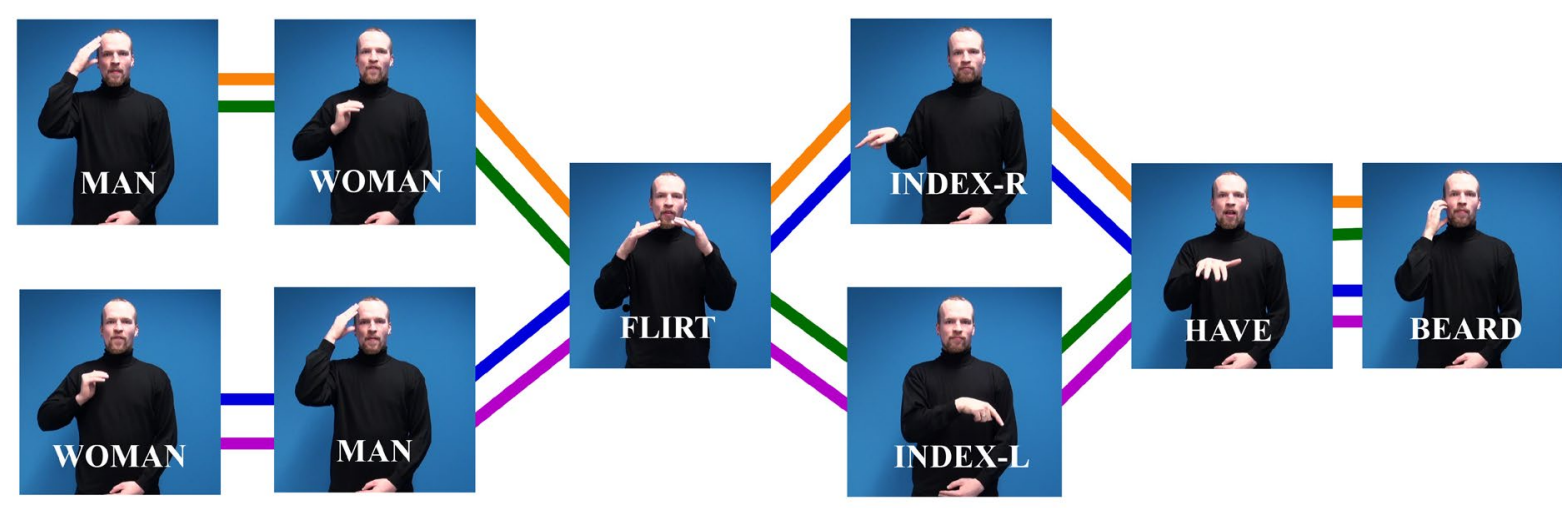

$\hookleftarrow$ CONDITION $1-$ CONDITION $2 \longrightarrow$ CONDITION $3 \longrightarrow$ CONDITION 4 
Appendix B: Chapter $1 \& 2-$ Complete list of stimulus material

The table presents all 40 sentence sets (in the rightmost column) used as stimulus material in Chapter 1 and 2. The sentences are given in glosses of German Sign Language sentences (bold-faced) with an English translation of the glosses below each sentence.

Conditions from Chapter 1 are shown in the column Condition while Chapter 2 is based on the direction of the INDEX sign column.

\begin{tabular}{|c|c|c|c|c|}
\hline Set & $\begin{array}{l}\text { Condi } \\
\text {-tion } \\
\text { (Chap- } \\
\text { ter 1) }\end{array}$ & $\begin{array}{l}\text { Congru- } \\
\text { ence of the } \\
\text { disambigua } \\
\text {-ting sign }\end{array}$ & $\begin{array}{c}\text { Direction } \\
\text { of the } \\
\text { INDEX sign } \\
\text { (Condition } \\
\text { Chapter 2) }\end{array}$ & $\begin{array}{c}\text { Stimulus material: } \\
\text { Glosses of German Sign Language sentences } \\
\text { (with English translation) }\end{array}$ \\
\hline 1 & 4 & $\begin{array}{l}\text { congruent } \\
\text { incongruent } \\
\text { incongruent } \\
\text { congruent }\end{array}$ & $\begin{array}{l}\text { ipsilateral } \\
\text { contralateral } \\
\text { ipsilateral } \\
\text { contralateral }\end{array}$ & $\begin{array}{l}\text { KIND MUTTER KÜSS. IX } \mathbf{X}_{3 \mathrm{a}} \text { IMMER FRECH } \\
\text { CHILD MOTHER KISS. IX ALWAYS CHEEKY. } \\
\text { KIND MUTTER KÜSS. IX } \mathbf{X}_{3 \mathrm{~b}} \text { IMMER FRECH. } \\
\text { CHILD MOTHER KISS. IX } \mathrm{X}_{3 \mathrm{~b}} \text { ALWAYS CHEEKY. } \\
\text { MUTTER KIND KÜSS. IX } \mathbf{X}_{3 \mathrm{a}} \text { IMMER FRECH. } \\
\text { MOTHER CHILD KISS. IX } \mathrm{X}_{3 \mathrm{a}} \text { ALWAYS CHEEKY. } \\
\text { MUTTER KIND KÜSS. IX } \mathbf{X}_{3 \mathrm{~b}} \text { IMMER FRECH. } \\
\text { MOTHER CHILD KISS. IX ALWAYS CHEEKY. }\end{array}$ \\
\hline 2 & 2 & $\begin{array}{l}\text { congruent } \\
\text { incongruent } \\
\text { incongruent } \\
\text { congruent }\end{array}$ & $\begin{array}{l}\text { ipsilateral } \\
\text { contralateral } \\
\text { ipsilateral } \\
\text { contralateral }\end{array}$ & $\begin{array}{l}\text { FRAU MANN TREFF. IX } \mathbf{X}_{3 \mathrm{a}} \text { WIEDER SCHWANGER. } \\
\text { WOMAN MAN MEET. IX } \mathrm{X}_{3 \mathrm{a}} \text { AGAIN PREGNANT. } \\
\text { FRAU MANN TREFF. IX } \mathbf{X}_{3 \mathrm{~b}} \text { WIEDER SCHWANGER. } \\
\text { WOMAN MAN MEET. IX } \mathrm{X}_{3 \mathrm{~b}} \text { AGAIN PREGNANT. } \\
\text { MANN FFRAU TREFF. IX } \mathbf{X}_{3 \mathrm{a}} \text { WIEDER SCHWANGER. } \\
\text { MAN WOMAN MEET. IX } \mathrm{X}_{3 \mathrm{a}} \text { AGAIN PREGNANT. } \\
\text { MANN FFRAU TREFF. IX } \mathbf{X}_{3 \mathrm{~B}} \text { WIEDER SCHWANGER. } \\
\text { MAN WOMAN MEET. IX } \mathrm{X}_{3 \mathrm{~b}} \text { AGAIN PREGNANT. }\end{array}$ \\
\hline 3 & 2 & $\begin{array}{l}\text { congruent } \\
\text { incongruent } \\
\text { incongruent } \\
\text { congruent }\end{array}$ & $\begin{array}{l}\text { ipsilateral } \\
\text { contralateral } \\
\text { ipsilateral } \\
\text { contralateral }\end{array}$ & $\begin{array}{l}\text { COWBOY KIND SUCH. IX } \mathbf{X}_{3 \mathrm{a}} \text { DA SCHNURRBART. } \\
\text { COWBOY CHILD SEARCH. IX } \mathrm{X}_{3 \mathrm{a}} \text { HAVE MOUSTACHE. } \\
\text { COWBOY KIND SUCH. IX } \mathbf{X}_{3 \mathrm{~b}} \text { DA SCHNURRBART. } \\
\text { COWBOY CHILD SEARCH. IX } \\
\text { KIND COWBOY MOUSTACHE. } \\
\text { CHILD COWBOY SEARCH. IX IX } \mathbf{X}_{3 \mathrm{a}} \text { DA SCHNURRBART. } \\
\text { KIND COWBOY SUCH. IX } \mathbf{X}_{3 \mathrm{~b}} \text { DA SCHNURRBART. } \\
\text { CHILD COWBOY SEARCH. IX } \mathrm{X}_{3 \mathrm{~b}} \text { HAVE MOUSTACHE. }\end{array}$ \\
\hline
\end{tabular}




\begin{tabular}{|c|c|c|c|c|}
\hline 4 & 1 & $\begin{array}{l}\text { congruent } \\
\text { incongruent } \\
\text { incongruent } \\
\text { congruent }\end{array}$ & $\begin{array}{l}\text { ipsilateral } \\
\text { contralateral } \\
\text { ipsilateral } \\
\text { contralateral }\end{array}$ & $\begin{array}{l}\text { KÖNIG HEXE KENNENLERN. IX }{ }_{3 a} \text { LAND REGIER. } \\
\text { KING WITCH GET-TO-KNOW. IX } \text { Xa }_{3 \mathrm{a}} \text { COUNTRY RULE. } \\
\text { KÖNIG HEXE KENNENLERN. IX } \mathbf{X}_{3 \mathrm{~b}} \text { LAND REGIER. } \\
\text { KING WITCH GET-TO-KNOW. IX } \mathrm{X}_{3 \mathrm{~b}} \text { COUNTRY RULE. } \\
\text { HEXE KÖNIG KENNENLERN. IX } \mathbf{X}_{3 \mathrm{a}} \text { LAND REGIER. } \\
\text { WITCH KING GET-TO-KNOW. IX }{ }_{3 \mathrm{a}} \text { COUNTRY RULE. } \\
\text { HEXE KÖNIG KENNENLERN. IX } \mathbf{X}_{3 \mathrm{~b}} \text { LAND REGIER. } \\
\text { WITCH KING GET-TO-KNOW. IX } \mathrm{X}_{3 \mathrm{~b}} \text { COUNTRY RULE. }\end{array}$ \\
\hline 5 & 1 & $\begin{array}{l}\text { congruent } \\
\text { incongruent } \\
\text { incongruent } \\
\text { congruent }\end{array}$ & $\begin{array}{l}\text { ipsilateral } \\
\text { contralateral } \\
\text { ipsilateral } \\
\text { contralateral }\end{array}$ & 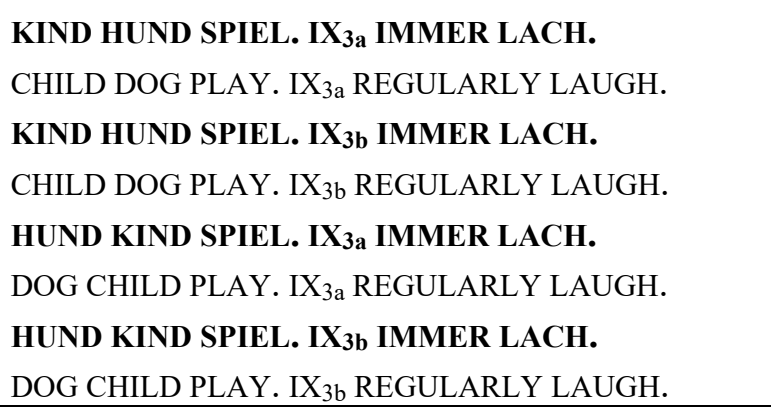 \\
\hline 6 & 1 & $\begin{array}{l}\text { congruent } \\
\text { incongruent } \\
\text { incongruent } \\
\text { congruent }\end{array}$ & $\begin{array}{l}\text { ipsilateral } \\
\text { contralateral } \\
\text { ipsilateral } \\
\text { contralateral }\end{array}$ & 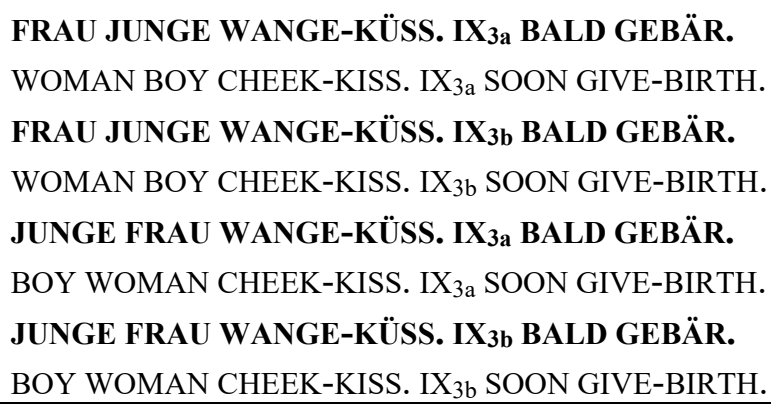 \\
\hline 7 & 1 & $\begin{array}{l}\text { congruent } \\
\text { incongruent } \\
\text { incongruent } \\
\text { congruent }\end{array}$ & $\begin{array}{l}\text { ipsilateral } \\
\text { contralateral } \\
\text { ipsilateral } \\
\text { contralateral }\end{array}$ & 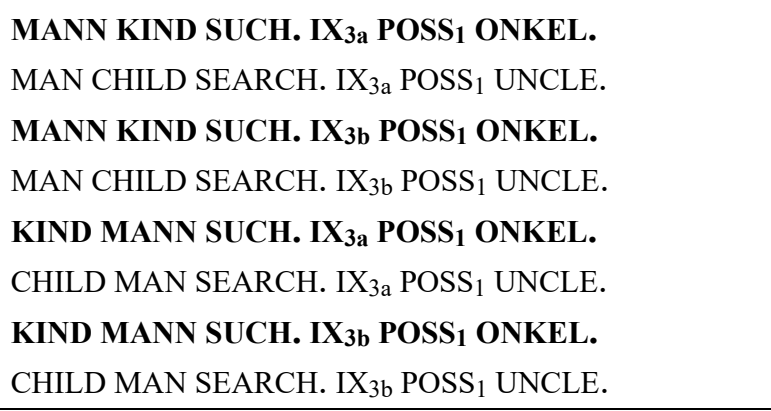 \\
\hline 8 & 1 & $\begin{array}{l}\text { congruent } \\
\text { incongruent } \\
\text { incongruent } \\
\text { congruent }\end{array}$ & $\begin{array}{l}\text { ipsilateral } \\
\text { contralateral } \\
\text { ipsilateral } \\
\text { contralateral }\end{array}$ & 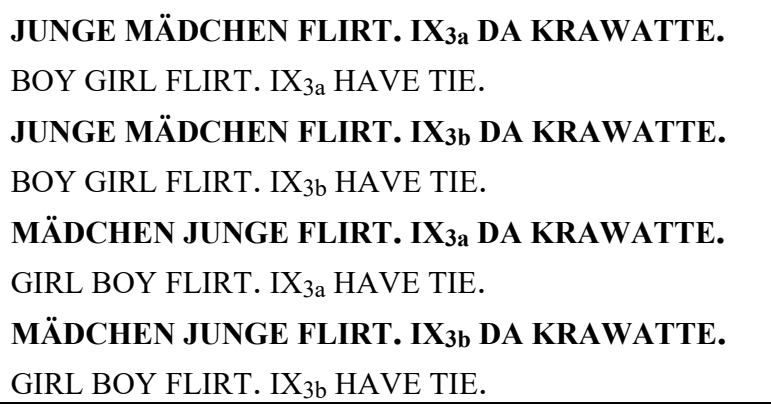 \\
\hline 9 & 1 & $\begin{array}{l}\text { congruent } \\
\text { incongruent } \\
\text { incongruent } \\
\text { congruent }\end{array}$ & $\begin{array}{l}\text { ipsilateral } \\
\text { contralateral } \\
\text { ipsilateral } \\
\text { contralateral }\end{array}$ & $\begin{array}{l}\text { OPA JUNGE GRÜß. IX } \mathbf{X}_{3 \mathrm{a}} \text { TÄGLICH PFEIFE-RAUCH. } \\
\text { GRANDPA BOY GREET. IX } \mathrm{X}_{3 \mathrm{a}} \text { DAILY PIPE-SMOKE. } \\
\text { OPA JUNGE GRÜß. IX } \mathbf{X}_{3 \mathrm{~b}} \text { TÄGLICH PFEIFE-RAUCH. } \\
\text { GRANDPA BOY GREET. IX } \mathrm{X}_{3 \mathrm{~b}} \text { DAILY PIPE-SMOKE. } \\
\text { JUNGE OPA GRÜß. IX } \mathbf{X}_{\mathbf{3 a}} \text { TÄGLICH PFEIFE-RAUCH. } \\
\text { BOY GRANDPA GREET. IX } \mathrm{X}_{3 \mathrm{a}} \text { DAILY PIPE-SMOKE. } \\
\text { JUNGE OPA GRÜß. IX } \mathbf{X}_{\mathbf{3 b}} \text { TÄGLICH PFEIFE-RAUCH. } \\
\text { BOY GRANDPA GREET. IX } \text { X }_{3 \mathrm{~b}} \text { DAILY PIPE-SMOKE. }\end{array}$ \\
\hline
\end{tabular}




\begin{tabular}{|c|c|c|c|c|}
\hline 10 & 1 & $\begin{array}{l}\text { congruent } \\
\text { incongruent } \\
\text { incongruent } \\
\text { congruent }\end{array}$ & $\begin{array}{l}\text { Ipsilateral } \\
\text { contralateral } \\
\text { ipsilateral } \\
\text { contralateral }\end{array}$ & 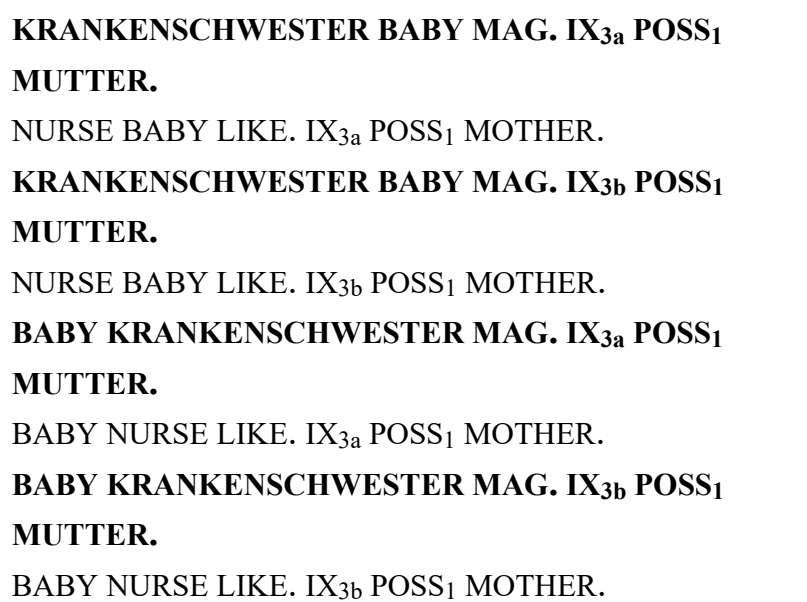 \\
\hline 11 & 1 & $\begin{array}{l}\text { congruent } \\
\text { incongruent } \\
\text { incongruent } \\
\text { congruent }\end{array}$ & $\begin{array}{l}\text { ipsilateral } \\
\text { contralateral } \\
\text { ipsilateral } \\
\text { contralateral }\end{array}$ & 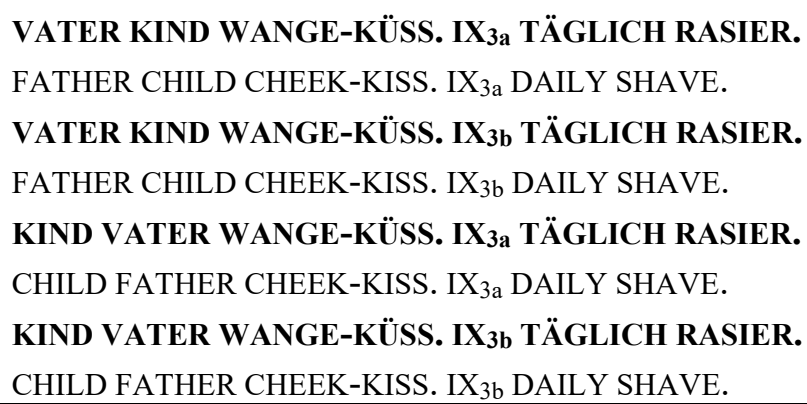 \\
\hline 12 & 1 & $\begin{array}{l}\text { congruent } \\
\text { incongruent } \\
\text { incongruent } \\
\text { congruent }\end{array}$ & $\begin{array}{l}\text { ipsilateral } \\
\text { contralateral } \\
\text { ipsilateral } \\
\text { contralateral }\end{array}$ & $\begin{array}{l}\text { BABY OMA SEH. IX } \mathbf{X}_{3 \mathrm{a}} \text { IMMER NUCKEL. } \\
\text { BABY GRANDMA SEE. IX } \\
\text { BAB ALWAYS SUCK. } \\
\text { BMA SEH. IX } \mathbf{X}_{3 \mathrm{~b}} \text { IMMER NUCKEL. } \\
\text { BABY GRANDMA SEE. IX } 3 \mathrm{~b} \text { ALWAYS SUCK. } \\
\text { OMA BABY SEH. IX } \mathbf{X}_{3 \mathrm{a}} \text { IMMER NUCKEL. } \\
\text { GRANDMA BABY SEE. IX } \mathrm{X}_{3 \mathrm{a}} \text { ALWAYS SUCK. } \\
\text { OMA BABY SEH. IX } \mathbf{X}_{3 \mathrm{~b}} \text { IMMER NUCKEL. } \\
\text { GRANDMA BABY SEE. IX } \mathrm{X}_{3 \mathrm{~b}} \text { ALWAYS SUCK. }\end{array}$ \\
\hline 13 & 1 & $\begin{array}{l}\text { incongruent } \\
\text { incongruent }\end{array}$ & $\begin{array}{l}\text { ipsilateral } \\
\text { contralateral } \\
\text { ipsilateral } \\
\text { contralateral }\end{array}$ & $\begin{array}{l}\text { KRANKENSCHWESTER SOLDAT HEIRAT. IX } \\
\text { Ja } \\
\text { JETZT SCHWANGER. } \\
\text { NURSE SOLDIER MARRY. IX }{ }_{3 \mathrm{a}} \text { NOW PREGNANT. } \\
\text { KRANKENSCHWESTER SOLDAT HEIRAT. IX } \\
\text { JbTZT SCHWANGER. } \\
\text { NURSE SOLDIER MARRY. IX } \\
\text { Sb NOW PREGNANT. } \\
\text { SOLDAT KRANKENSCHWESTER HEIRAT. IX } \\
\text { JETZT SCHWANGER. } \\
\text { SOLDIER NURSE MARRY. IX } \\
\text { SOLDAT NOW PREGNANT. } \\
\text { JETZT SCHWANGER. } \\
\text { SOLDIER NURSE MARRY. IX }{ }_{3 b} \text { NOW PREGNANT. }\end{array}$ \\
\hline 14 & 1 & $\begin{array}{l}\text { congruent } \\
\text { incongruent } \\
\text { incongruent } \\
\text { congruent }\end{array}$ & $\begin{array}{l}\text { ipsilateral } \\
\text { contralateral } \\
\text { ipsilateral } \\
\text { contralateral }\end{array}$ & $\begin{array}{l}\text { ARZT KIND SEH. IX } \mathbf{X}_{3 \mathrm{a}} \text { LANGE OPERIER. } \\
\text { DOCTOR CHILD SEE. IX } 3 \mathrm{3a} \text { LONG OPERATE. } \\
\text { ARZT KIND SEH. IX } \mathbf{X}_{3 \mathrm{~b}} \text { LANGE OPERIER. } \\
\text { DOCTOR CHILD SEE. IX } \mathrm{X}_{3 \mathrm{~b}} \text { LONG OPERATE. } \\
\text { KIND ARZT SEH. IX } \mathbf{X}_{3 \mathrm{a}} \text { LANGE OPERIER. } \\
\text { CHILD DOCTOR SEE. IX } \mathrm{X}_{3 \mathrm{a}} \text { LONG OPERATE. } \\
\text { KIND ARZT SEH. IX I } \text { LANGE OPERIER. } \\
\text { CHILD DOCTOR SEE. IX } \mathrm{X}_{3 \mathrm{~b}} \text { LONG OPERATE. }\end{array}$ \\
\hline
\end{tabular}




\begin{tabular}{|c|c|c|c|c|}
\hline 15 & 1 & $\begin{array}{l}\text { congruent } \\
\text { incongruent } \\
\text { incongruent } \\
\text { congruent }\end{array}$ & $\begin{array}{l}\text { ipsilateral } \\
\text { contralateral } \\
\text { ipsilateral } \\
\text { contralateral }\end{array}$ & 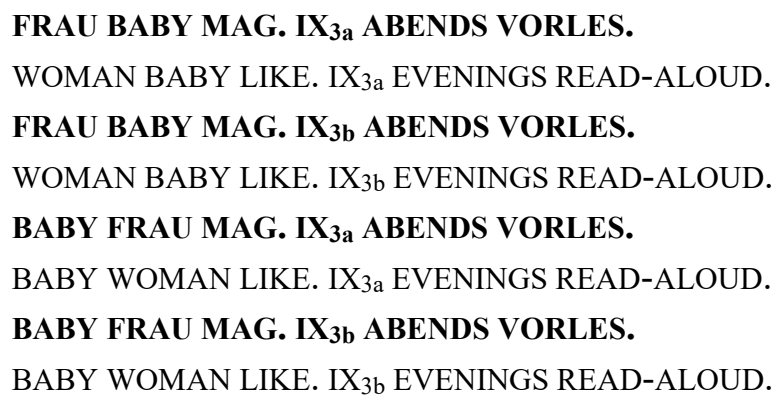 \\
\hline 16 & 1 & $\begin{array}{l}\text { congruent } \\
\text { incongruent } \\
\text { incongruent } \\
\text { congruent }\end{array}$ & $\begin{array}{l}\text { ipsilateral } \\
\text { contralateral } \\
\text { ipsilateral } \\
\text { contralateral }\end{array}$ & 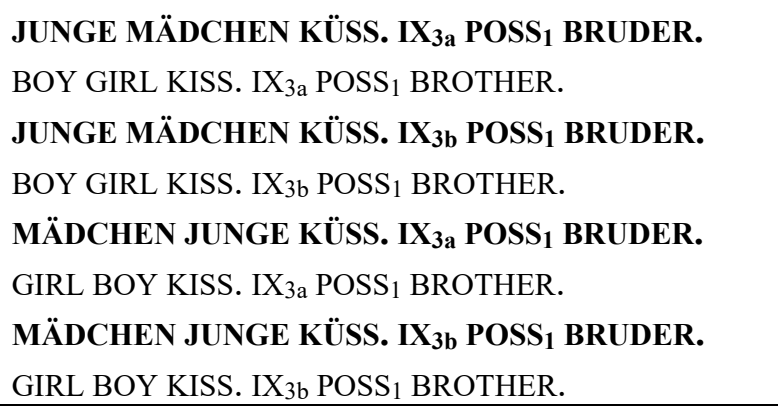 \\
\hline 17 & 1 & incongruent & contralateral & $\begin{array}{l}\text { FRAU KIND KENNENLERN. IX } \mathbf{3}_{3 \mathrm{a}} \text { RESTAURANT } \\
\text { ARBEIT. } \\
\text { WOMAN CHILD GET-TO-KNOW. IX }{ }_{3 \mathrm{a}} \text { RESTAURANT } \\
\text { WORK. } \\
\text { FRAU KIND KENNENLERN. IX } \mathbf{X}_{3 \mathrm{~b}} \text { RESTAURANT } \\
\text { ARBEIT. } \\
\text { WOMAN CHILD GET-TO-KNOW. IX }{ }_{3 \mathrm{~b}} \text { RESTAURANT } \\
\text { WORK. } \\
\text { KIND FRAU KENNENLERN. IX } \mathbf{X}_{3 \mathrm{a}} \text { RESTAURANT } \\
\text { ARBEIT. } \\
\text { CHILD WOMAN GET-TO-KNOW. IX } 3 \mathrm{3} \text { RESTAURANT } \\
\text { WORK. } \\
\text { KIND FRAU KENNENLERN. IX } \mathbf{X}_{3 \mathrm{~b}} \text { RESTAURANT } \\
\text { ARBEIT. } \\
\text { CHILD WOMAN GET-TO-KNOW. IX } \\
\text { WORK. }\end{array}$ \\
\hline 18 & 1 & incongruent & ipsilateral & 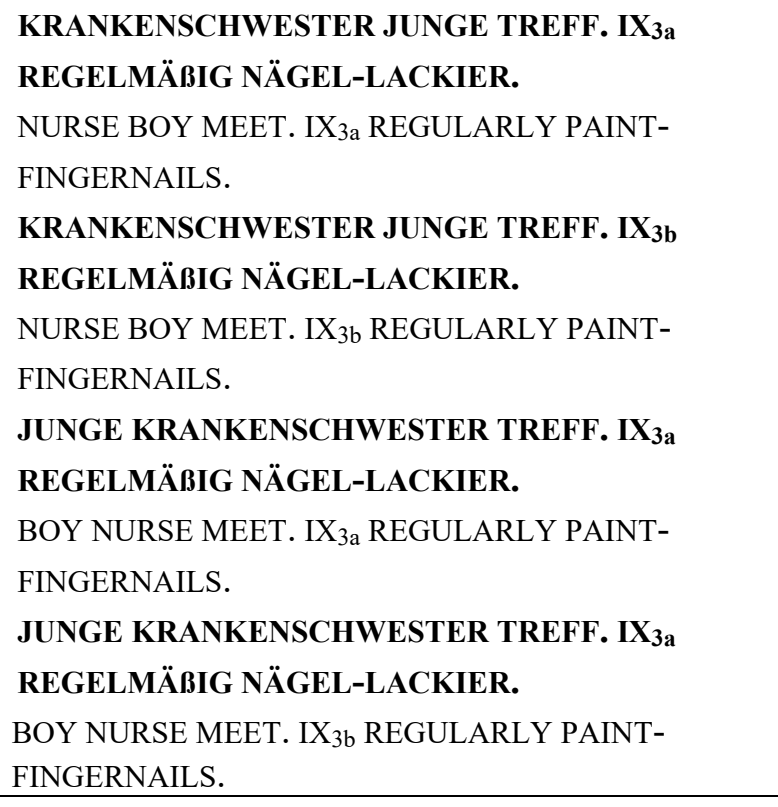 \\
\hline
\end{tabular}




\begin{tabular}{|c|c|c|c|c|}
\hline 19 & 1 & $\begin{array}{l}\text { congruent } \\
\text { incongruent } \\
\text { incongruent } \\
\text { congruent }\end{array}$ & $\begin{array}{l}\text { ipsilateral } \\
\text { contralateral } \\
\text { ipsilateral } \\
\text { contralateral }\end{array}$ & 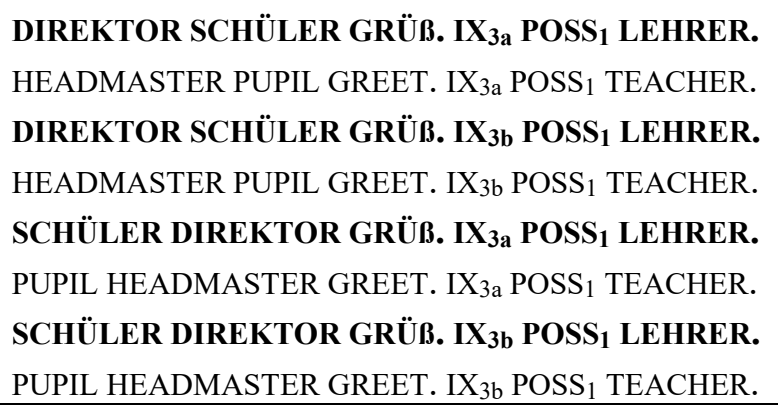 \\
\hline 20 & 1 & $\begin{array}{l}\text { congruent } \\
\text { incongruent } \\
\text { incongruent } \\
\text { congruent }\end{array}$ & $\begin{array}{l}\text { ipsilateral } \\
\text { contralateral } \\
\text { ipsilateral } \\
\text { contralateral }\end{array}$ & 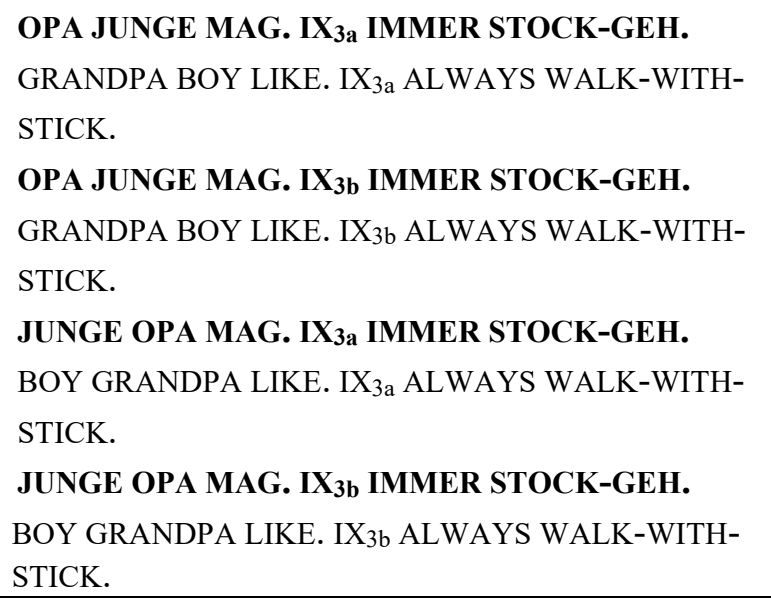 \\
\hline 21 & 3 & $\begin{array}{l}\text { congruent } \\
\text { incongruent } \\
\text { incongruent } \\
\text { congruent }\end{array}$ & $\begin{array}{l}\text { ipsilateral } \\
\text { contralateral } \\
\text { ipsilateral } \\
\text { contralateral }\end{array}$ & 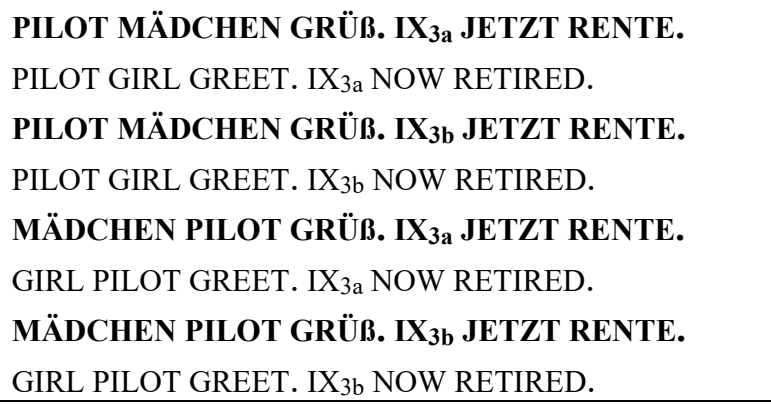 \\
\hline 22 & 4 & $\begin{array}{l}\text { congruent } \\
\text { incongruent } \\
\text { incongruent } \\
\text { congruent }\end{array}$ & $\begin{array}{l}\text { ipsilateral } \\
\text { contralateral } \\
\text { ipsilateral } \\
\text { contralateral }\end{array}$ & $\begin{array}{l}\text { LEHRER SCHÜLER KENN. IX } \mathbf{X}_{3 \mathrm{a}} \text { DAMALS STRENG. } \\
\text { TEACHER PUPIL KNOW. IX } 3 \mathrm{a} \text { BACK-THEN STRICT. } \\
\text { LEHRER SCHÜLER KENN. IX } \mathbf{X}_{3 \mathrm{~b}} \text { DAMALS STRENG. } \\
\text { TEACHER PUPIL KNOW. IX } 3 \text { Bb BACK-THEN STRICT. } \\
\text { SCHÜLER LEHRER KENN. IX } \mathbf{X}_{3 \mathrm{a}} \text { DAMALS STRENG. } \\
\text { PUPIL TEACHER KNOW. IX } 3 \mathrm{a} \text { BACK-THEN STRICT. } \\
\text { SCHÜLER LEHRER KENN. IX } \\
\text { PUPIL DAMALS STRENG. }\end{array}$ \\
\hline 23 & 1 & $\begin{array}{l}\text { congruent } \\
\text { incongruent } \\
\text { incongruent } \\
\text { congruent }\end{array}$ & $\begin{array}{l}\text { ipsilateral } \\
\text { contralateral } \\
\text { ipsilateral } \\
\text { contralateral }\end{array}$ & $\begin{array}{l}\text { PRIESTER OMA TREFF. IX } \mathbf{X}_{\mathbf{3}} \text { DA BART. } \\
\text { PRIEST GRANDMA MEET. IX } \mathrm{X}_{3 \mathrm{a}} \text { HAVE BEARD. } \\
\text { PRIESTER OMA TREFF. IX } \mathbf{3}_{\mathbf{3 b}} \text { DA BART. } \\
\text { PRIEST GRANDMA MEET. IX } \mathrm{X}_{3 \mathrm{~b}} \text { HAVE BEARD. } \\
\text { OMA PRIESTER TREFF. IX } \mathbf{X}_{\mathbf{3 a}} \text { DA BART. } \\
\text { GRANDMA PRIEST MEET. IX } \mathrm{X}_{3 \mathrm{a}} \text { HAVE BEARD. } \\
\text { OMA PRIESTER TREFF. IX } \mathbf{X}_{\mathbf{3 b}} \text { DA BART. } \\
\text { GRANDMA PRIEST MEET. IX IX HAVE BEARD. }\end{array}$ \\
\hline
\end{tabular}




\begin{tabular}{|c|c|c|c|c|}
\hline 24 & 1 & $\begin{array}{l}\text { congruent } \\
\text { incongruent } \\
\text { incongruent } \\
\text { congruent }\end{array}$ & $\begin{array}{l}\text { ipsilateral } \\
\text { contralateral } \\
\text { ipsilateral } \\
\text { contralateral }\end{array}$ & $\begin{array}{l}\text { COWBOY HUND SEH. IX } \mathbf{X}_{\mathbf{3}} \text { DA PISTOLE. } \\
\text { COWBOY DOG SEE. IX } \mathrm{X}_{3 \mathrm{a}} \text { HAVE GUN. } \\
\text { COWBOY HUND SEH. IX } \mathbf{X}_{\mathbf{3 b}} \text { DA PISTOLE. } \\
\text { COWBOY DOG SEE. IX } \mathrm{X}_{3 \mathrm{~b}} \text { HAVE GUN. } \\
\text { HUND COWBOY SEH. IX } \mathbf{X}_{3 \mathrm{a}} \text { DA PISTOLE. } \\
\text { DOG COWBOY SEE. IX } \mathrm{I}_{3 \mathrm{a}} \text { HAVE GUN. } \\
\text { HUND COWBOY SEH. IX } \mathbf{X}_{\mathbf{3 b}} \text { DA PISTOLE. } \\
\text { DOG COWBOY SEE. IX } \mathrm{I}_{3 \mathrm{~b}} \text { HAVE GUN. }\end{array}$ \\
\hline 25 & 1 & $\begin{array}{l}\text { congruent } \\
\text { incongruent }\end{array}$ & $\begin{array}{l}\text { ipsilateral } \\
\text { contralateral } \\
\text { ipsilateral }\end{array}$ & $\begin{array}{l}\text { MÄDCHEN JUNGE WANGE-KÜSS. IX } 3 \text { IM IMER } \\
\text { LIPPENSTIFT-AUFTRAG. } \\
\text { GIRL BOY CHEEK-KISS. IX } \\
\text { ON. } \\
\text { MÄDCHEN JUNGE WANGE-KÜSS. IX } 3 \text { bb IMMER } \\
\text { LIPPENSTIFT-AUFTRAG. } \\
\text { GIRL BOY CHEEK-KISS. IX } \\
\text { ON. } \\
\text { JUNGE MÄDCHEN WANGE-KÜSS. IX } \mathbf{X}_{3 \mathrm{a}} \text { IMMER } \\
\text { LIPPENSTIFT-AUFTRAG. } \\
\text { BOY GIRL CHEEK-KISS. IX } \\
\text { ON. } \\
\text { JUNGE ML̈WAYS PUT-LIPSTICK- } \\
\text { LIPPENSTIFT-AUFTRAG. } \\
\text { BOY GIRL CHEEK-KISS. IX } \\
\text { ON. }\end{array}$ \\
\hline 26 & 1 & $\begin{array}{l}\text { congruent } \\
\text { incongruent } \\
\text { incongruent } \\
\text { congruent }\end{array}$ & $\begin{array}{l}\text { ipsilateral } \\
\text { contralateral } \\
\text { ipsilateral } \\
\text { contralateral }\end{array}$ & 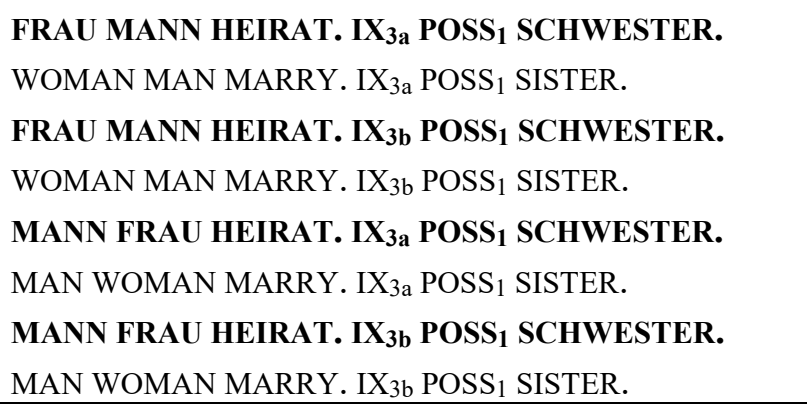 \\
\hline 27 & 4 & incongruent & ipsilateral & 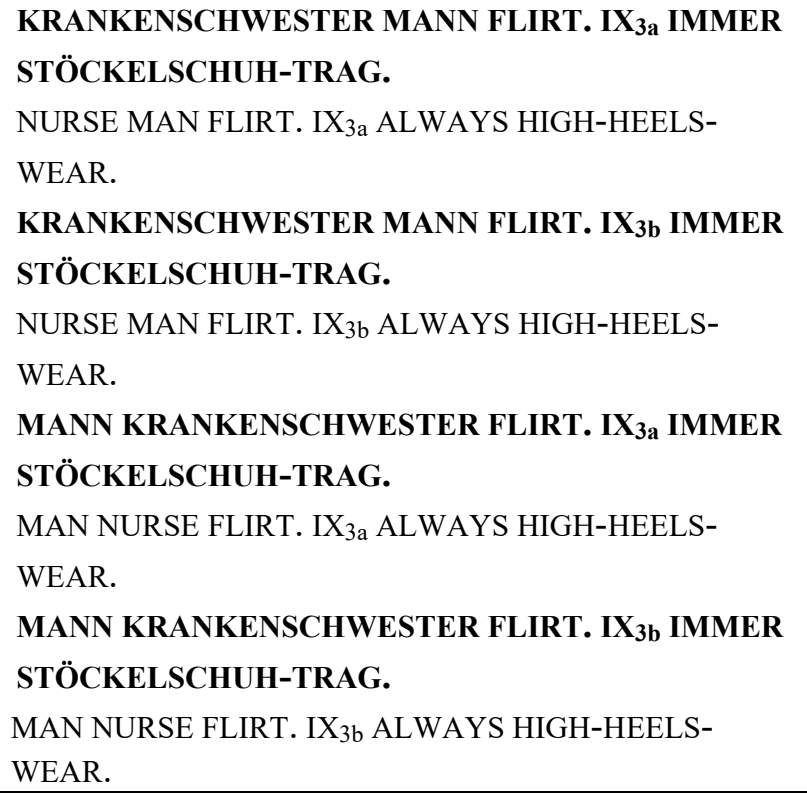 \\
\hline
\end{tabular}




\begin{tabular}{|c|c|c|c|c|}
\hline 28 & 1 & incongruent & $\begin{array}{l}\text { contralateral } \\
\text { ipsilateral } \\
\text { contralateral }\end{array}$ & 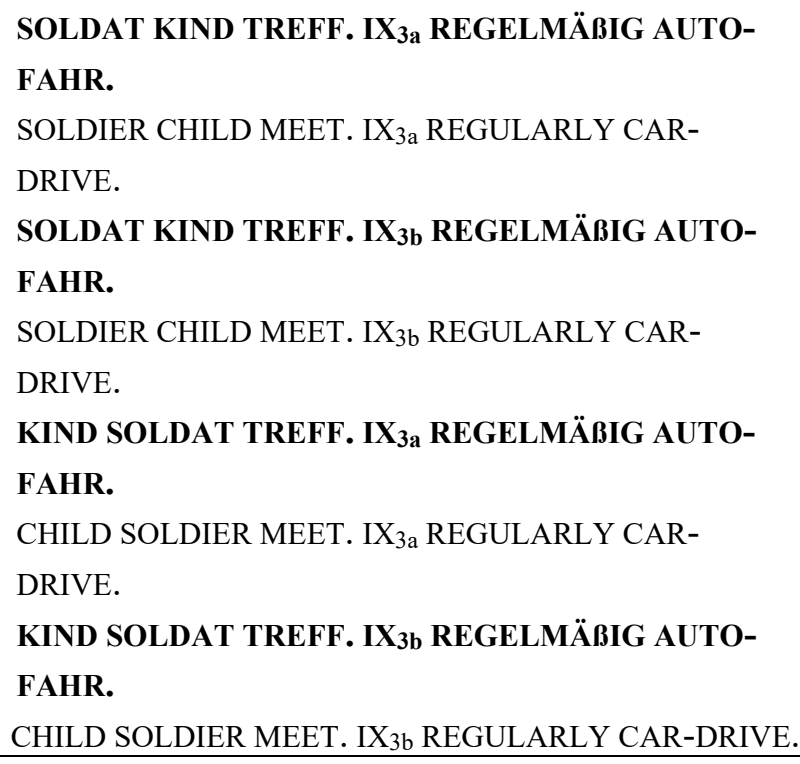 \\
\hline 29 & 1 & $\begin{array}{l}\text { congruent } \\
\text { incongruent } \\
\text { incongruent } \\
\text { congruent }\end{array}$ & $\begin{array}{l}\text { ipsilateral } \\
\text { contralateral } \\
\text { ipsilateral } \\
\text { contralateral }\end{array}$ & $\begin{array}{l}\text { JUNGE KATZE SEH. IX } \text { 3a }_{\mathbf{a}} \text { JETZT GEBÄRD. } \\
\text { BOY CAT SEE. IX } \mathrm{X}_{3 \mathrm{a}} \text { NOW SIGN. } \\
\text { JUNGE KATZE SEH. IX } \mathbf{X}_{\mathbf{3 b}} \text { JETZT GEBÄRD. } \\
\text { BOY CAT SEE. IX } \mathrm{X}_{3 \mathrm{~b}} \text { NOW SIGN. } \\
\text { KATZE JUNGE SEH. IX } \mathbf{X}_{3 \mathrm{a}} \text { JETZT GEBÄRD. } \\
\text { CAT BOY SEE. IX } \mathrm{X}_{3 \mathrm{a}} \text { NOW SIGN. } \\
\text { KATZE JUNGE SEH. IX } \mathbf{X}_{\mathbf{3 b}} \text { JETZT GEBÄRD. } \\
\text { CAT BOY SEE. IX } \mathrm{X}_{3 \mathrm{~b}} \text { NOW SIGN. }\end{array}$ \\
\hline 30 & 1 & $\begin{array}{l}\text { congruent } \\
\text { incongruent } \\
\text { incongruent } \\
\text { congruent }\end{array}$ & $\begin{array}{l}\text { ipsilateral } \\
\text { contralateral } \\
\text { ipsilateral } \\
\text { contralateral }\end{array}$ & 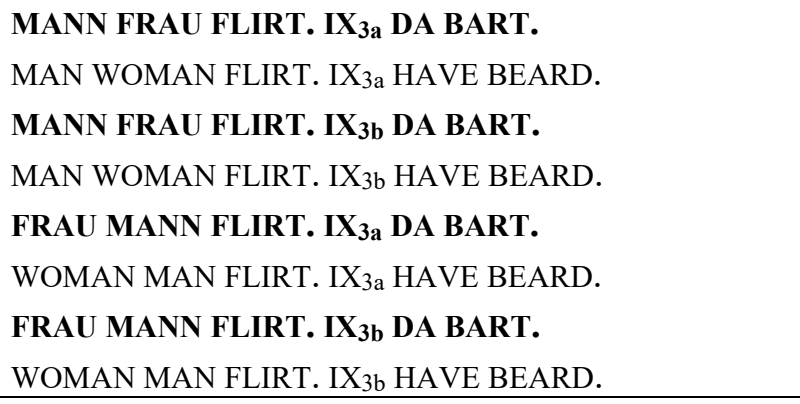 \\
\hline 31 & 1 & $\begin{array}{l}\text { congruent } \\
\text { incongruent } \\
\text { incongruent } \\
\text { congruent }\end{array}$ & $\begin{array}{l}\text { ipsilateral } \\
\text { contralateral } \\
\text { ipsilateral } \\
\text { contralateral }\end{array}$ & 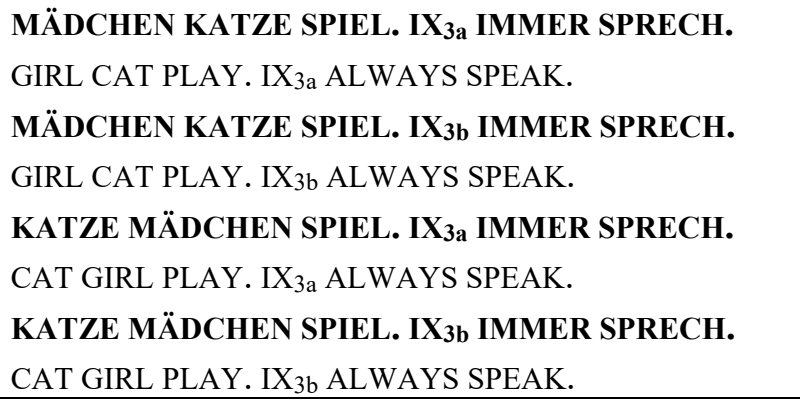 \\
\hline 32 & 1 & $\begin{array}{l}\text { congruent } \\
\text { incongruent } \\
\text { incongruent } \\
\text { congruent }\end{array}$ & $\begin{array}{l}\text { ipsilateral } \\
\text { contralateral } \\
\text { ipsilateral } \\
\text { contralateral }\end{array}$ & 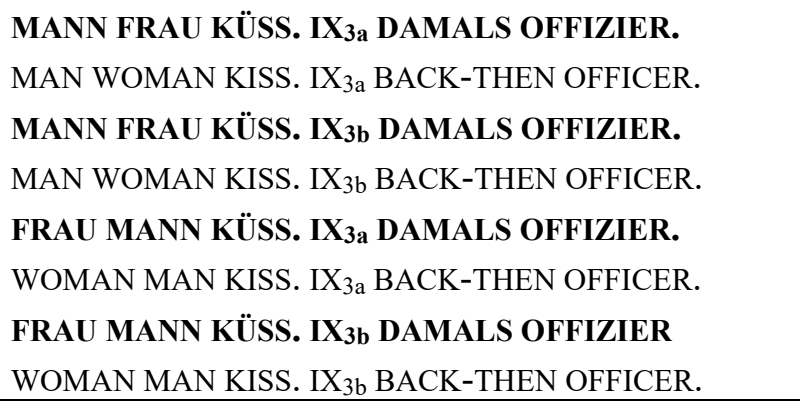 \\
\hline
\end{tabular}




\begin{tabular}{|c|c|c|c|c|}
\hline 33 & 1 & $\begin{array}{l}\text { congruent } \\
\text { incongruent } \\
\text { incongruent }\end{array}$ & $\begin{array}{l}\text { ipsilateral } \\
\text { contralateral } \\
\text { ipsilateral } \\
\text { contralateral }\end{array}$ & 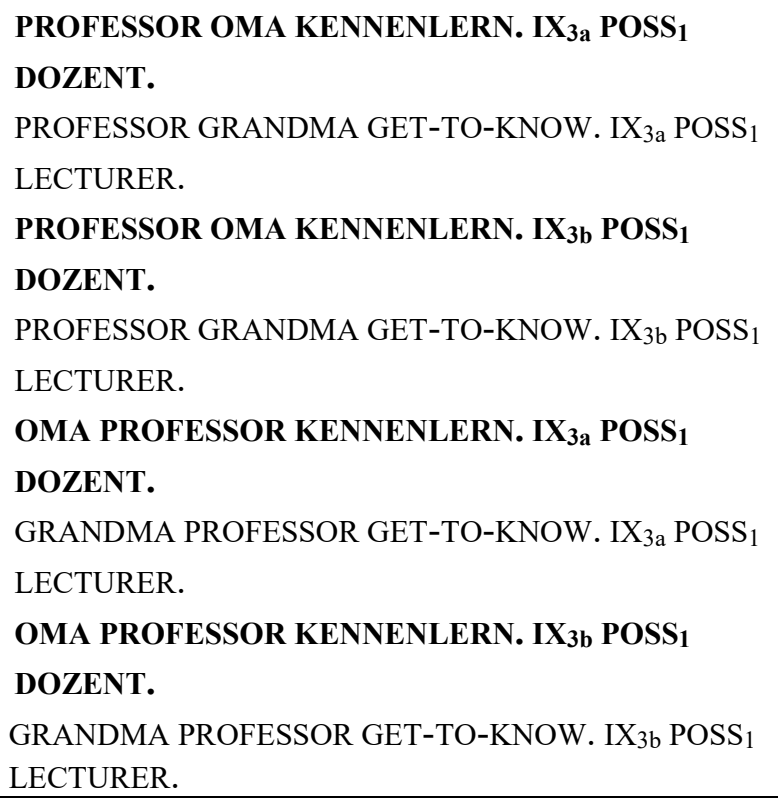 \\
\hline 34 & 4 & $\begin{array}{l}\text { congruent } \\
\text { incongruent } \\
\text { incongruent } \\
\text { congruent }\end{array}$ & $\begin{array}{l}\text { ipsilateral } \\
\text { contralateral } \\
\text { ipsilateral } \\
\text { contralateral }\end{array}$ & $\begin{array}{l}\text { JUNGE KATZE SPIEL. IX } \mathbf{X}_{3 \mathrm{a}} \text { OFT LES. } \\
\text { BOY CAT PLAY. IX } \mathrm{X}_{3 \mathrm{a}} \text { OFTEN READ. } \\
\text { JUNGE KATZE SPIEL. IX } \mathbf{X}_{3 \mathrm{~b}} \text { OFT LES. } \\
\text { BOY CAT PLAY. IX } \mathrm{X}_{3 \mathrm{~b}} \text { OFTEN READ. } \\
\text { KATZE JUNGE SPIEL. IX } \mathbf{X}_{3 \mathrm{a}} \text { OFT LES. } \\
\text { CAT BOY PLAY. IX } \mathrm{X}_{3 \mathrm{a}} \text { OFTEN READ. } \\
\text { KATZE JUNGE SPIEL. IX } \mathbf{X}_{3 \mathrm{~b}} \text { OFT LES. } \\
\text { CAT BOY PLAY. IX } \\
\text { 3b OFTEN READ. }\end{array}$ \\
\hline 35 & 1 & $\begin{array}{l}\text { congruent } \\
\text { incongruent } \\
\text { incongruent } \\
\text { congruent }\end{array}$ & $\begin{array}{l}\text { ipsilateral } \\
\text { contralateral } \\
\text { ipsilateral } \\
\text { contralateral }\end{array}$ & 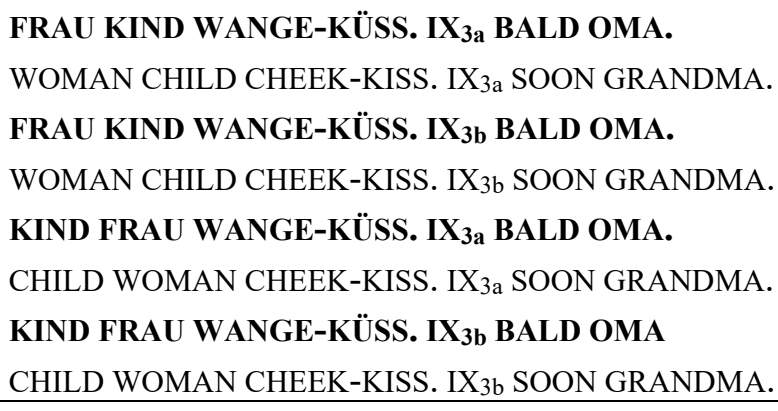 \\
\hline 36 & 1 & $\begin{array}{l}\text { congruent } \\
\text { incongruent }\end{array}$ & $\begin{array}{l}\text { ipsilateral } \\
\text { contralateral } \\
\text { ipsilateral } \\
\text { contralateral }\end{array}$ & 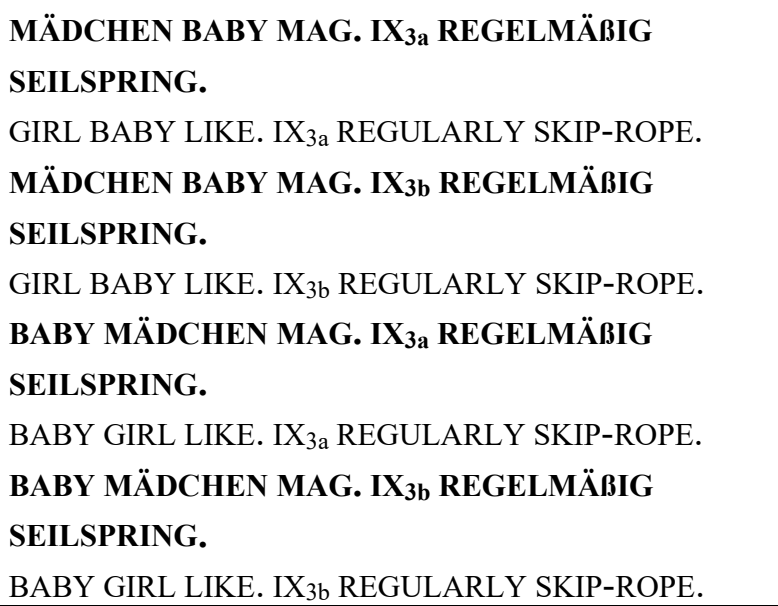 \\
\hline
\end{tabular}




\begin{tabular}{|c|c|c|c|c|}
\hline 37 & 1 & $\begin{array}{l}\text { congruent } \\
\text { incongruent } \\
\text { incongruent } \\
\text { congruent }\end{array}$ & $\begin{array}{l}\text { ipsilateral } \\
\text { contralateral } \\
\text { ipsilateral } \\
\text { contralateral }\end{array}$ & 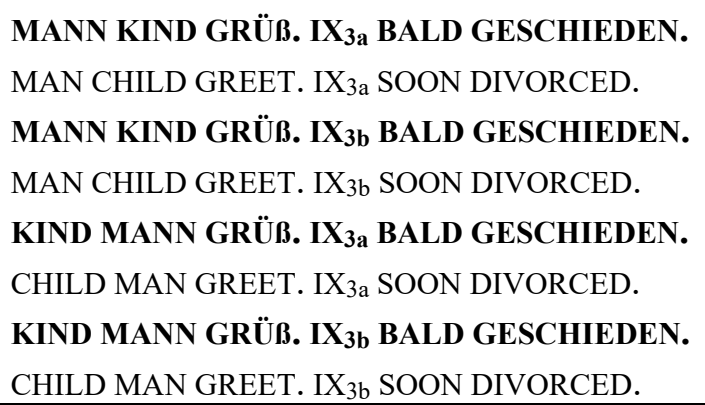 \\
\hline 38 & 1 & $\begin{array}{l}\text { congruent } \\
\text { incongruent } \\
\text { incongruent } \\
\text { congruent }\end{array}$ & $\begin{array}{l}\text { ipsilateral } \\
\text { contralateral } \\
\text { ipsilateral } \\
\text { contralateral }\end{array}$ & 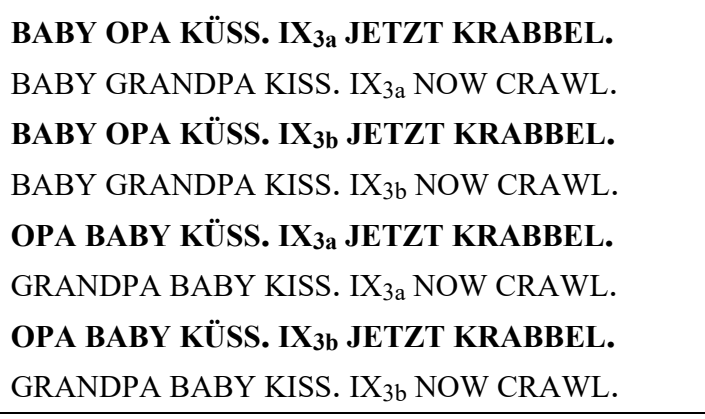 \\
\hline 39 & 1 & $\begin{array}{l}\text { congruent } \\
\text { incongruent } \\
\text { incongruent } \\
\text { congruent }\end{array}$ & $\begin{array}{l}\text { ipsilateral } \\
\text { contralateral } \\
\text { ipsilateral } \\
\text { contralateral }\end{array}$ & 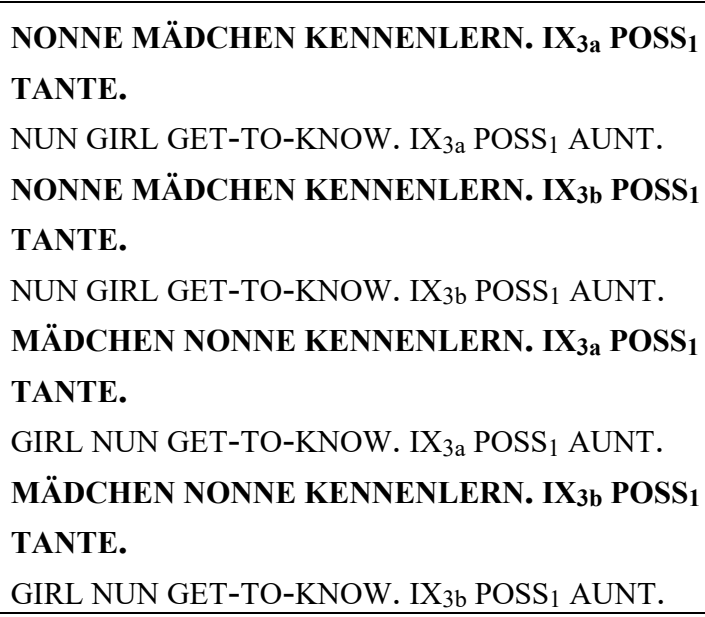 \\
\hline 40 & 1 & $\begin{array}{l}\text { congruent } \\
\text { incongruent } \\
\text { incongruent } \\
\text { congruent }\end{array}$ & $\begin{array}{l}\text { ipsilateral } \\
\text { contralateral } \\
\text { ipsilateral } \\
\text { contralateral }\end{array}$ & 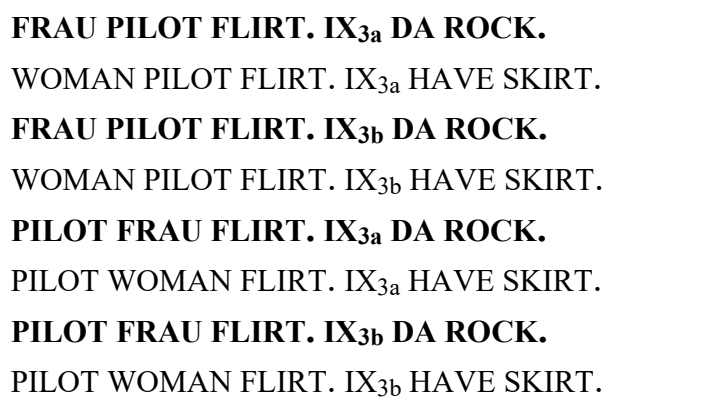 \\
\hline
\end{tabular}


Appendix C: Chapter 2 - Schematic illustration of the regions of interest (ROI)

Schematic illustration of the regions of interest (ROIs): There are six regions in total with 3 regions per hemisphere consisting of six electrodes each. Regions are marked by same type of lines: dotted - fronto-central region; dashed - centro-temporal region; dotdashed parietal-occipital region.

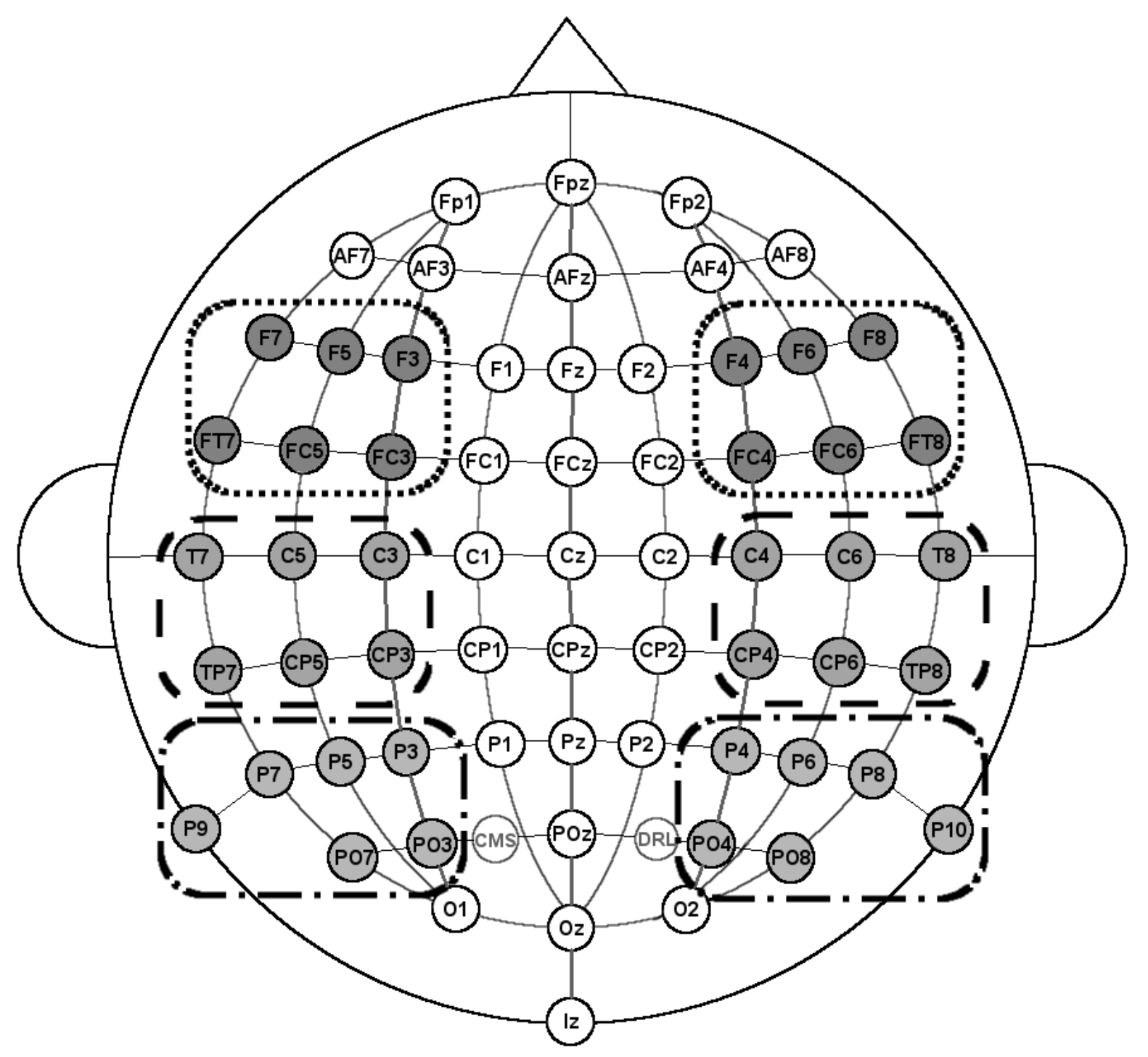


Appendix D: Chapter 3 - Scene stills of videos for each condition and each continuation type

Video stills for each sign of the sentence are shown linearly from left to right. The first sentence varies in the overt localization of the referents. Empty frames substitute the absent INDEX sign in the conditions subject, object and no localization. However, these frames were only added in the figure to highlight the difference between conditions, but they do not occur in the videos. The second sentence was the same for all four conditions. Figure 1 presents a sentence set from the subject continuation type and figure 2 shows the object continuation type.

Figure 1: Sentences continuing with the subject

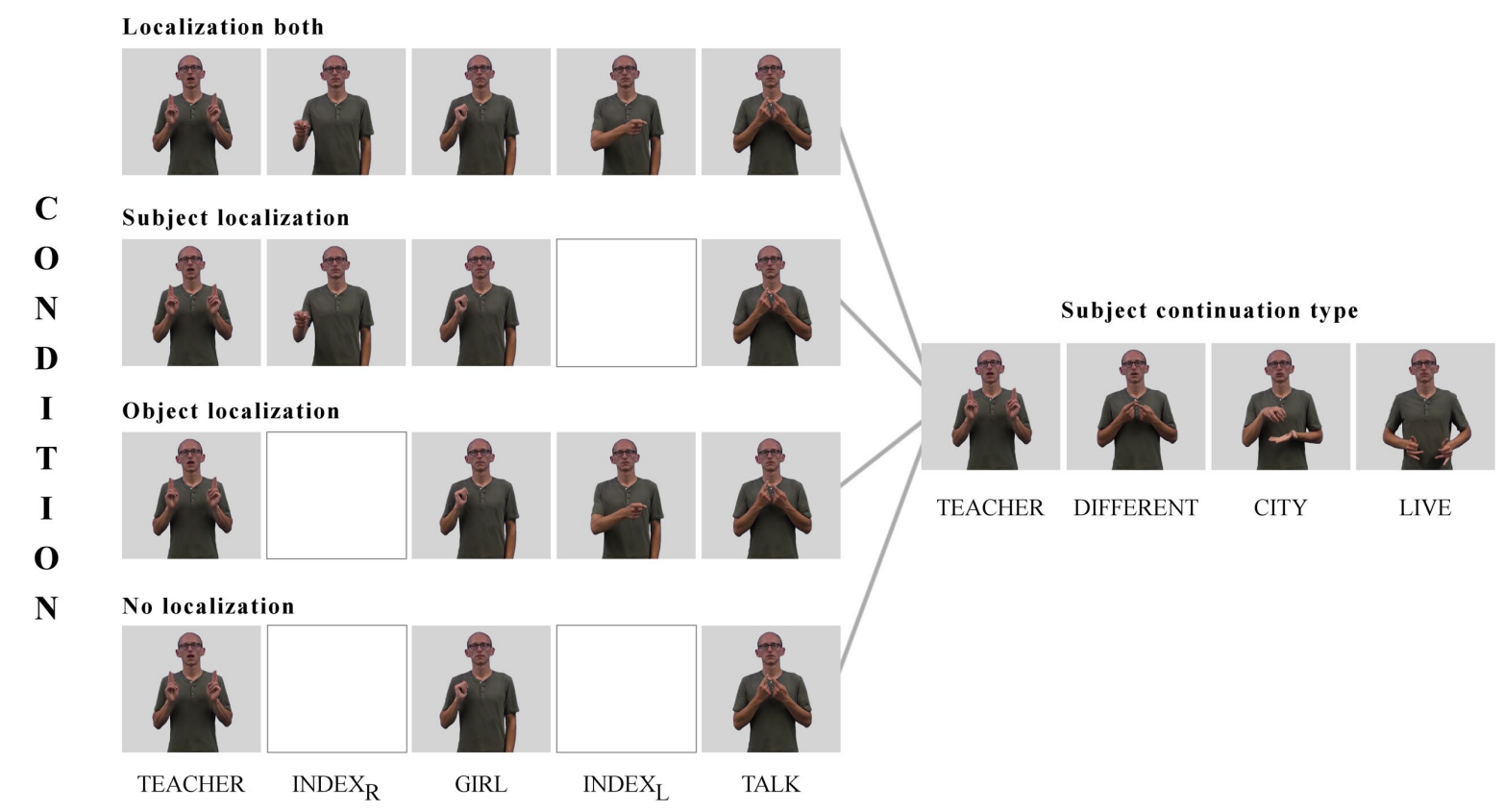


Figure 2: Sentences continuing with the object

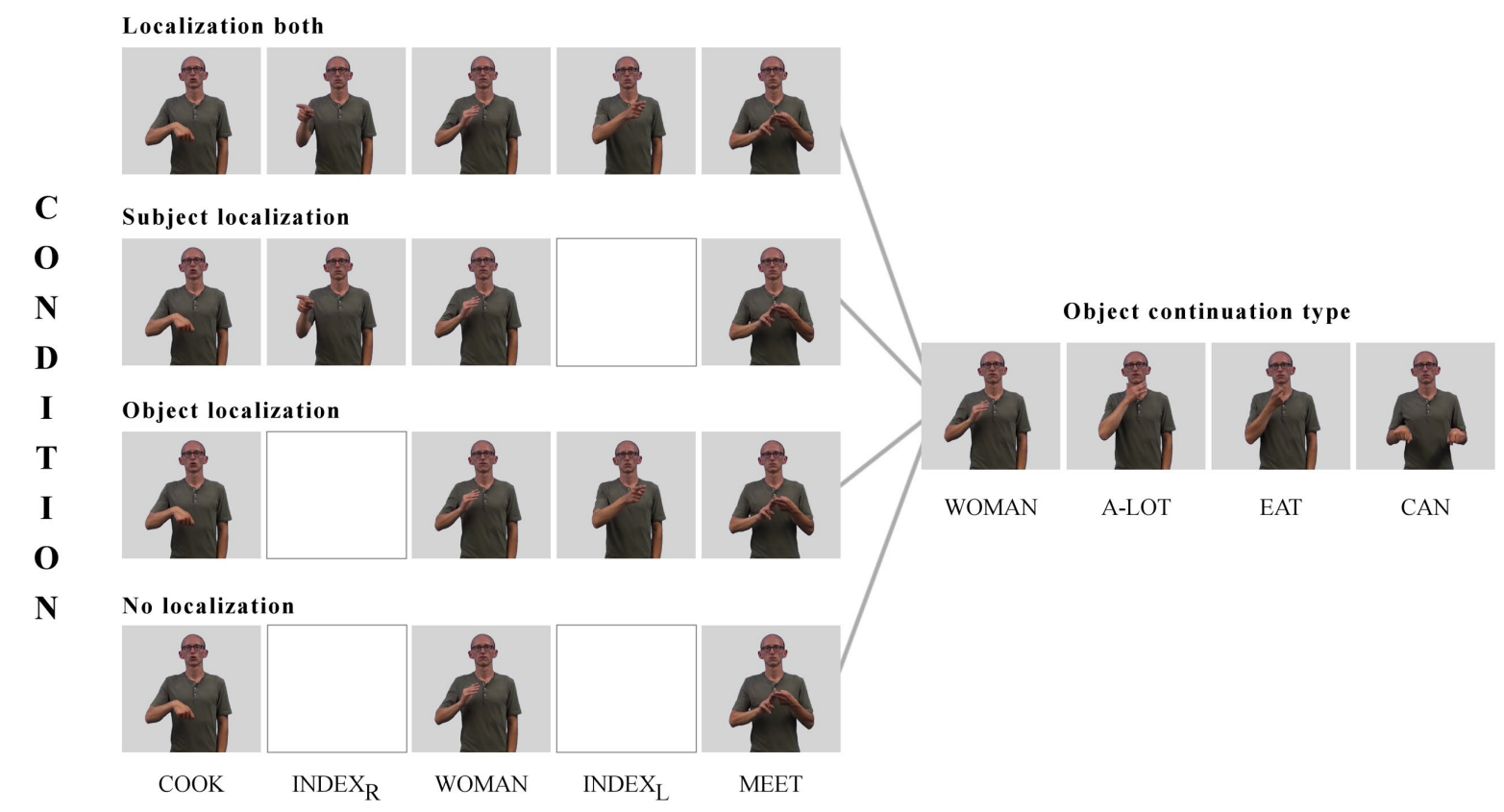


Appendix E: Chapter 3 - Complete list of stimulus material

The stimulus material for each continuation type (object continuation (Table 1), subject continuation (Table 2) and the practice items (Table 3) are given here. In each table, the first column presents the name of the video for each sentence. In the second column, the condition of each sentence is indicated (both = localization of subject and object; subject = only subject localization; object $=$ only object localization; none $=$ no (overt) localization) . The rightmost column presents the sentence set as glosses of German Sign Language (boldfaced) with English glosses below. The lines within each sentence were only added to highlight the differences between sentences of different conditions and to facilitate the readers' understanding. In Table 3, the third column indicates the continuation type the sentence set was presented in (object $=$ object continuation type; subject $=$ subject continuation type).

Table 1: Stimulus material for sentences continuing with the object

\begin{tabular}{|c|c|c|}
\hline $\begin{array}{l}\text { Video } \\
\text { name }\end{array}$ & $\begin{array}{l}\text { Condi } \\
\text {-tion }\end{array}$ & Sentence set \\
\hline S01so & both & $\begin{array}{l}\text { OPA IX } \mathbf{I X}_{\mathbf{R}} \text { OMA IX } \mathbf{I}_{\mathbf{L}} \text { KÜSS. OMA INNERLICH SEHR FROH. } \\
\text { GRANDPA IX } \mathrm{X}_{\mathrm{R}} \text { GRANDMA IX IX KISS. GRANDMA INTERNAL VERY HAPPY. }\end{array}$ \\
\hline S01s & subject & $\begin{array}{l}\text { OPA IX } \mathbf{X}_{\mathbf{R}} \text { OMA } \\
\text { GRANDPA IX KÜSS. OMA INNERLICH SEHR FROH. } \\
\text { GRANDMA__ KISS. GRANDMA INTERNAL VERY HAPPY. }\end{array}$ \\
\hline S01o & object & $\begin{array}{l}\text { OPA__ OMA IX } \mathbf{I X}_{\mathbf{L}} \text { KÜSS. OMA INNERLICH SEHR FROH. } \\
\text { GRANDPA__ GRANDMA IX KISS. GRANDMA INTERNAL VERY HAPPY. }\end{array}$ \\
\hline S01n & none & $\begin{array}{l}\text { OPA _ OMA } \_ \text {KÜSS. OMA INNERLICH SEHR FROH. } \\
\text { GRANDPA__ GRANDMA _ KISS. GRANDMA INTERNAL VERY HAPPY. }\end{array}$ \\
\hline S02so & both & $\begin{array}{l}\text { FRAU IX } \text { K }_{\mathbf{R}} \text { KÖNIG IX } \mathbf{I}_{\mathbf{L}} \text { KÜSS. KÖNIG MEHR AUFMERKSAMKEIT BRAUCH. } \\
\text { WOMAN IX } \\
\end{array}$ \\
\hline S02s & subject & $\begin{array}{l}\text { FRAU IX } \mathbf{R}_{\mathbf{R}} \text { KÖNIG_— KÜSS. KÖNIG MEHR AUFMERKSAMKEIT BRAUCH. } \\
\text { WOMAN IX KING_ KISS. KING MORE ATTENTION NEED. }^{-}\end{array}$ \\
\hline S02o & object & $\begin{array}{l}\text { FRAU _ KÖNIG IX } \text { L }_{\mathbf{L}} \text { KÜSS. KÖNIG MEHR AUFMERKSAMKEIT BRAUCH. } \\
\text { WOMAN _ KING IX }{ }_{\text {L }} \text { KISS. KING MORE ATTENTION NEED. }\end{array}$ \\
\hline S02n & none & $\begin{array}{ll}\text { FRAU _ KÖNIG__ KÜSS. KÖNIG MEHR AUFMERKSAMKEIT BRAUCH. } \\
\text { WOMAN_KING__ KISS. KING MORE ATTENTION NEED. }\end{array}$ \\
\hline S03so & both & $\begin{array}{l}\text { KRANKENSCHWESTER IX } \mathbf{R}_{\mathbf{R}} \text { MANN IX } \mathrm{X}_{\mathrm{L}} \text { KÜSS. MANN BODEN HART UMFALL. } \\
\text { NURSE } \mathrm{I}_{\mathrm{R}} \text { MAN IX } \mathrm{X}_{\mathrm{L}} \text { KISS. MAN FLOOR HARD FALL-DOWN. }\end{array}$ \\
\hline
\end{tabular}




\begin{tabular}{|c|c|c|}
\hline S03s & subject & $\begin{array}{l}\text { KRANKENSCHWESTER IX } \text { R }_{R} \text { MANN__ KÜSS. MANN BODEN HART UMFALL. } \\
\text { NURSE IX } \text { I }_{R} \text { MAN__ KISS. MAN FLOOR HARD FALL-DOWN. }\end{array}$ \\
\hline S03o & object & 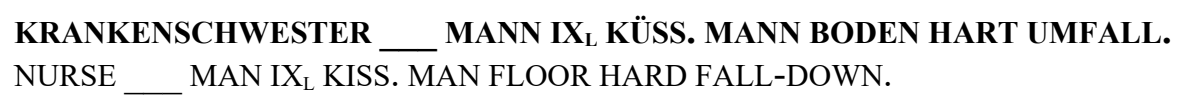 \\
\hline S03n & none & $\begin{array}{l}\text { KRANKENSCHWESTER _ MANN _ KÜSS. MANN BODEN HART UMFALL. } \\
\text { NURSE _ MAN __ KISS. MAN FLOOR HARD FALL-DOWN. }\end{array}$ \\
\hline S04so & both & $\begin{array}{l}\text { JUNGE IX } \mathbf{R}_{\mathbf{R}} \text { MÄDCHEN IX } \mathbf{L} \text { KÜSS. MÄDCHEN LIEBESBRIEF SCHREIB. } \\
\text { BOY IX } \text { GIRL IX }_{L} \text { KISS. GIRL LOVE LETTER WRITE. }\end{array}$ \\
\hline S04s & subject & $\begin{array}{l}\text { JUNGE IX } \mathbf{R}_{\mathbf{R}} \text { MÄDCHEN } \\
\text { BOY IX } \mathrm{X}_{\mathrm{R}} \text { GIRL } \_ \text {KÏSS. MÄDCHEN LIEBESBRIEF SCHREIB. GIRL LOVE LETTER WRITE. }\end{array}$ \\
\hline S04o & object & 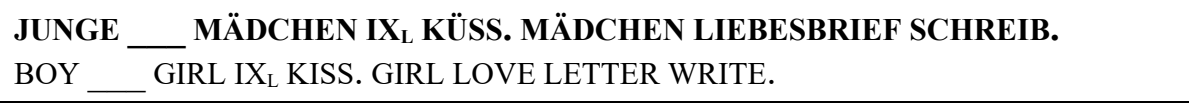 \\
\hline S04n & none & $\begin{array}{l}\text { JUNGE __ MÄDCHEN__ KÜSS. MÄDCHEN LIEBESBRIEF SCHREIB. } \\
\text { BOY __ GIRL__ KISS. GIRL LOVE LETTER WRITE. }\end{array}$ \\
\hline S05so & both & $\begin{array}{l}\text { JÄGERN IX } \mathbf{R}_{\mathbf{R}} \text { HEXE IX } \mathbf{L}_{\mathbf{L}} \text { TREFF. HEXE TIERE SCHÜTZ WÜNSCH. } \\
\text { HUNTER IXR WITCH IX } \mathrm{X}_{\mathrm{L}} \text { MEET. WITCH ANIMALS PROTECT WISH. }\end{array}$ \\
\hline S05s & subject & $\begin{array}{l}\text { JÄGERN IX } \mathbf{R}_{\mathbf{R}} \text { HEXE — TREFF. HEXE TIERE SCHÜTZ WÜNSCH. } \\
\text { HUNTER IX } \mathrm{X}_{\mathrm{R}} \text { WITCH__ MEET. WITCH ANIMALS PROTECT WISH. }\end{array}$ \\
\hline S05o & object & $\begin{array}{l}\text { JÄGERN _ HEXE IX } \text { X L }_{\mathbf{L}} \text { TREFF. HEXE TIERE SCHÜTZ WÜNSCH. } \\
\text { HUNTER — WITCH IX }{ }_{L} \text { MEET. WITCH ANIMALS PROTECT WISH. }\end{array}$ \\
\hline S05n & none & $\begin{array}{l}\text { JÄGERN _ _ HEXE__ TREFF. HEXE TIERE SCHÜTZ WÜNSCH. } \\
\text { HUNTER__ WITCH__ MEET. WITCH ANIMALS PROTECT WISH. }\end{array}$ \\
\hline S06so & both & $\begin{array}{l}\text { OMA IX }_{\mathbf{R}} \text { PRIESTER IX } \mathbf{X}_{\mathrm{L}} \text { TREFF. PRIESTER BIBEL LESEN MAG(LIEB). } \\
\text { GRANDMA IX }_{R} \text { PRIEST IX }{ }_{L} \text { MEET. PRIEST BIBLE READ LOVE. }\end{array}$ \\
\hline S06s & subject & $\begin{array}{l}\text { OMA IX } \mathbf{X}_{\mathbf{R}} \text { PRIESTER __ TREFF. PRIESTER BIBEL LESEN MAG(LIEB). } \\
\text { GRANDMA IX }_{R} \text { PRIEST__ MEET. PRIEST BIBLE READ LOVE. }\end{array}$ \\
\hline S06o & object & $\begin{array}{l}\text { OMA _ PRIESTER IX }{ }_{\text {L TREFF. PRIESTER BIBEL LESEN MAG(LIEB). }} \\
\text { GRANDMA__ PRIEST IX } X_{L} \text { MEET. PRIEST BIBLE READ LOVE. }\end{array}$ \\
\hline S06n & none & $\begin{array}{l}\text { OMA _ PRIESTER _ TREFF. PRIESTER BIBEL LESEN MAG(LIEB). } \\
\text { GRANDMA_ _ PRIEST _ MEET. PRIEST BIBLE READ LOVE. }\end{array}$ \\
\hline S07so & both & $\begin{array}{l}\text { LEHRER IX } \text { R }_{\text {CLOWN IX }} \text { TREFF. CLOWN KINDERGRUPPE BETREU. } \\
\text { TEACHER IX }_{\text {R }} \text { CLOWN IX }{ }_{L} \text { MEET. CLOWN CHILDREN GROUP LOOK-AFTER. }\end{array}$ \\
\hline S07s & subject & $\begin{array}{l}\text { LEHRER IX }_{\mathbf{R}} \text { CLOWN _ TREFF. CLOWN KINDERGRUPPE BETREU. } \\
\text { TEACHER IX }_{R} \text { CLOWN _ MEET. CLOWN CHILDREN GROUP LOOK-AFTER. }\end{array}$ \\
\hline S07o & object & 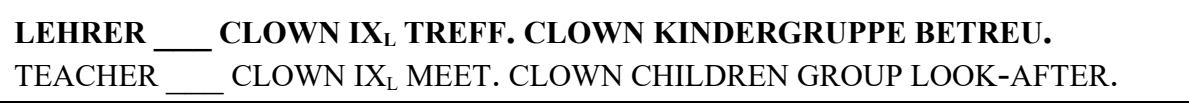 \\
\hline S07n & none & $\begin{array}{l}\text { LEHRER__CLOWN__ TREFF. CLOWN KINDERGRUPPE BETREU. } \\
\text { TEACHER__CLOWN__ MEET. CLOWN CHILDREN GROUP LOOK-AFTER. }\end{array}$ \\
\hline S08so & both & $\begin{array}{l}\text { KOCH IX } \mathbf{X}_{\mathbf{R}} \text { FRAU IX } \mathbf{X}_{\mathbf{L}} \text { TREFF. FRAU VIEL ESS KANN. } \\
\text { COOK IX } \mathrm{X}_{\mathrm{R}} \text { WOMAN IX } \mathrm{X}_{\mathrm{L}} \text { MEET. WOMAN A-LOT EAT CAN. }\end{array}$ \\
\hline S08s & subject & $\begin{array}{l}\text { KOCH IX } \mathbf{X}_{\mathbf{R}} \text { FRAU _ TREFF. FRAU VIEL ESS KANN. } \\
\text { COOK IX WOMAN _ MEET. WOMAN A-LOT EAT CAN. }\end{array}$ \\
\hline S08o & object & $\begin{array}{l}\text { KOCH _ FRAU IX } \mathbf{X}_{\mathbf{L}} \text { TREFF. FRAU VIEL ESS KANN. } \\
\text { COOK__ WOMAN IX } \mathrm{X}_{\mathrm{L}} \text { MEET. WOMAN A-LOT EAT CAN. }\end{array}$ \\
\hline S08n & none & $\begin{array}{l}\text { KOCH _ FRAU__ TREFF. FRAU VIEL ESS KANN. } \\
\text { COOK_ WOMAN_ MEET. WOMAN A-LOT EAT CAN. }\end{array}$ \\
\hline
\end{tabular}




\begin{tabular}{|c|c|c|}
\hline S09so & both & $\begin{array}{l}\text { PILOT IX } \mathbf{X}_{\mathbf{R}} \text { MALER IX } \mathbf{X}_{\mathbf{L}} \text { KENNENLERN. MALER STADT GROß WOHN. } \\
\text { PILOT IX } \mathrm{X}_{R} \text { PAINTER IX } \mathrm{L}_{\mathrm{L}} \text { GET-TO-KNOW. PAINTER CITY BIG LIVE-IN. }\end{array}$ \\
\hline $\mathrm{S} 09 \mathrm{~s}$ & subject & $\begin{array}{l}\text { PILOT IX } \mathbf{X}_{\mathbf{R}} \text { MALER —— KENNENLERN. MALER STADT GROß WOHN. } \\
\text { PILOT IX }_{\mathrm{R}} \text { PAINTER_ GET-TO-KNOW. PAINTER CITY BIG LIVE-IN. }\end{array}$ \\
\hline S09o & object & 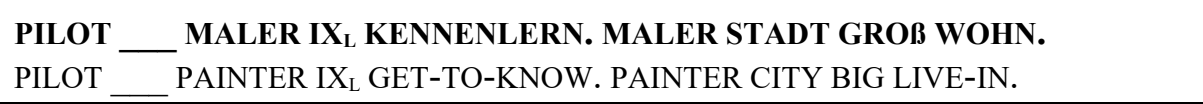 \\
\hline S09n & none & $\begin{array}{l}\text { PILOT _ MALER — KENNENLERN. MALER STADT GROß WOHN. } \\
\text { PILOT_ PAINTER_ GET-TO-KNOW. PAINTER CITY BIG LIVE-IN. }\end{array}$ \\
\hline S10so & both & $\begin{array}{l}\text { ARZT IX }{ }_{R} \text { KRANKENSCHWESTER IX } X_{L} \text { KENNENLERN. KRANKENSCHWESTER } \\
\text { VERSCHIEDENE KRANKHEITEN KENN. } \\
\text { DOCTOR IX } X_{R} \text { NURSE IX } X_{L} \text { GET-TO-KNOW. NURSE DIFFERENT DISEASES KNOW. }\end{array}$ \\
\hline S10s & subject & $\begin{array}{l}\text { ARZT IX }{ }_{\mathbf{R}} \text { KRANKENSCHWESTER _ KENNENLERN. KRANKENSCHWESTER } \\
\text { VERSCHIEDENE KRANKHEITEN KENN. } \\
\text { DOCTOR IX } X_{R} \text { NURSE__ GET-TO-KNOW. NURSE DIFFERENT DISEASES KNOW. }\end{array}$ \\
\hline S10o & object & $\begin{array}{l}\text { ARZT_ KRANKENSCHWESTER IX } X_{\text {L KENNENLERN. KRANKENSCHWESTER }} \\
\text { VERSCHIEDENE KRANKHEITEN KENN. } \\
\text { DOCTOR_ _ NURSE IX } X_{L} \text { GET-TO-KNOW. NURSE DIFFERENT DISEASES KNOW. }\end{array}$ \\
\hline S10n & none & $\begin{array}{l}\text { ARZT _ KRANKENSCHWESTER _ KENNENLERN. KRANKENSCHWESTER } \\
\text { VERSCHIEDENE KRANKHEITEN KENN. } \\
\text { DOCTOR_ NURSE__ GET-TO-KNOW. NURSE DIFFERENT DISEASES KNOW. }\end{array}$ \\
\hline S11so & both & $\begin{array}{l}\text { JUNGE IX } \mathbf{R}_{\mathbf{R}} \text { CLOWN IX } \mathbf{L}_{\mathbf{L}} \text { KENNENLERN. CLOWN VIELE WTZE ERZÄHL. } \\
\text { BOY IX } \mathrm{X}_{\mathrm{R}} \text { CLOWN IX } \mathrm{X}_{\mathrm{L}} \text { GET-TO-KNOW. CLOWN A-LOT JOKES TELL. }\end{array}$ \\
\hline $\mathrm{S} 11 \mathrm{~s}$ & subject & $\begin{array}{l}\text { JUNGE IX } \text { I }_{\mathbf{R}} \text { CLOWN _ KENNENLERN. CLOWN VIELE WTZE ERZÄHL. } \\
\text { BOY IX }{ }_{R} \text { CLOWN } \_ \text {GET-TO-KNOW. CLOWN A-LOT JOKES TELL. }\end{array}$ \\
\hline S11o & object & $\begin{array}{l}\text { JUNGE _CLOWN IX }{ }_{\text {L KENNENLERN. CLOWN VIELE WTZE ERZÄHL. }} \\
\text { BOY _ CLOWN IX } X_{L} \text { GET-TO-KNOW. CLOWN A-LOT JOKES TELL. }\end{array}$ \\
\hline S11n & none & $\begin{array}{l}\text { JUNGE _CLOWN _ KENNENLERN. CLOWN VIELE WTZE ERZÄHL. } \\
\text { BOY __LLWN__ GET-TO-KNOW. CLOWN A-LOT JOKES TELL. }\end{array}$ \\
\hline S12so & both & $\begin{array}{l}\text { MÄDCHEN IX } \mathbf{X}_{\mathbf{R}} \text { NONNE IX } \mathbf{X}_{L} \text { KENNENLERN. NONNE SCHÖNE AUGEN DA. } \\
\text { GIRL IX }_{R} \text { NUN IX } X_{L} \text { GET-TO-KNOW. NUN BEAUTIFUL EYES HAVE. }\end{array}$ \\
\hline S12s & subject & $\begin{array}{l}\text { MÄDCHEN IX } \text { R }_{\text {RONNE }} \text { KENNENLERN. NONNE SCHÖNE AUGEN DA. } \\
\text { GIRL IX } X_{R} \text { NUN__ GET-TO-KNOW. NUN BEAUTIFUL EYES HAVE. }\end{array}$ \\
\hline S12o & object & $\begin{array}{l}\text { MÄDCHEN _ NONNE IX } X_{L} \text { KENNENLERN. NONNE SCHÖNE AUGEN DA. } \\
\text { GIRL__ NUN IX } X_{L} \text { GET-TO-KNOW. NUN BEAUTIFUL EYES HAVE. }\end{array}$ \\
\hline $\mathrm{S} 12 \mathrm{n}$ & none & $\begin{array}{l}\text { MÄDCHEN _ NONNE } \_ \text {KENNENLERN. NONNE SCHÖNE AUGEN DA. } \\
\text { GIRL__ NUN _ GET-TO-KNOW. NUN BEAUTIFUL EYES HAVE. }\end{array}$ \\
\hline S13so & both & $\begin{array}{l}\text { SOLDAT IX } X_{R} \text { KRANKENSCHWESTER IX }{ }_{L} \text { FLIRT. KRANKENSCHWESTER VIEL } \\
\text { SPAß DA. } \\
\text { SOLDIER IX } X_{R} \text { NURSE IX }{ }_{L} \text { FLIRT. NURSE HAVE A-LOT FUN. }\end{array}$ \\
\hline $\mathrm{S} 13 \mathrm{~s}$ & subject & $\begin{array}{l}\text { SOLDAT IX } X_{R} \text { KRANKENSCHWESTER _ _ FLIRT. KRANKENSCHWESTER VIEL } \\
\text { SPAß DA. } \\
\text { SOLDIER IX } X_{R} \text { NURSE__ FLIRT. NURSE HAVE A-LOT FUN. }\end{array}$ \\
\hline S13o & object & $\begin{array}{l}\text { SOLDAT__ KRANKENSCHWESTER IX }{ }_{L} \text { FLIRT. KRANKENSCHWESTER VIEL } \\
\text { SPAß DA. } \\
\text { SOLDIER __ NURSE IX }{ }_{\text {L FLIRT. NURSE HAVE A-LOT FUN. }}\end{array}$ \\
\hline S13n & none & $\begin{array}{l}\text { SOLDAT__ KRANKENSCHWESTER__ FLIRT. KRANKENSCHWESTER VIEL } \\
\text { SPAß DA. _ } \\
\text { SOLDIER __ NURSE__ FLIRT. NURSE HAVE A-LOT FUN. }\end{array}$ \\
\hline
\end{tabular}




\begin{tabular}{|c|c|c|}
\hline S14so & both & $\begin{array}{l}\text { FRAU IX } \mathbf{X}_{\mathbf{R}} \text { POLIZIST IX } \mathbf{X}_{\mathbf{L}} \text { FLIRT. POLIZIST ZWEI BIER BESTELL. } \\
\text { WOMAN IX } X_{R} \text { POLICEMAN IX }{ }_{L} \text { FLIRT. POLICEMAN TWO BEER ORDER. }\end{array}$ \\
\hline S14s & subject & 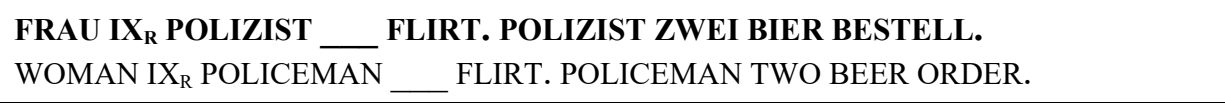 \\
\hline S14o & object & 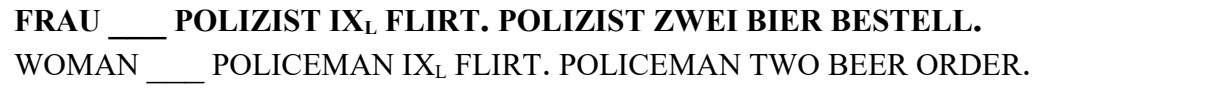 \\
\hline $\mathrm{S} 14 \mathrm{n}$ & none & $\begin{array}{l}\text { FRAU __ POLIZIST__ FLIRT. POLIZIST ZWEI BIER BESTELL. } \\
\text { WOMAN__ POLICEMAN __FLIRT. POLICEMAN TWO BEER ORDER. }\end{array}$ \\
\hline S15so & both & $\begin{array}{l}\text { MANN IX } \mathbf{R}_{\mathbf{R}} \text { HEXE IX } \mathbf{X}_{\mathbf{L}} \text { FLIRT. HEXE TANZPARTNER SUCH. } \\
\text { MAN IX } \mathrm{X}_{\mathrm{R}} \text { WITCH IX } \mathrm{L}_{\mathrm{L}} \text { FLIRT. WITCH DANCING PARTNER SEARCH. }\end{array}$ \\
\hline $\mathrm{S} 15 \mathrm{~s}$ & subject & $\begin{array}{l}\text { MANN IX } \mathbf{X}_{\mathbf{R}} \text { HEXE __ FLIRT. HEXE TANZPARTNER SUCH. } \\
\text { MAN IX } \mathrm{WITCH}_{\mathrm{R}} \text { FLIRT. WITCH DANCING PARTNER SEARCH. }\end{array}$ \\
\hline $\mathrm{S} 15 \mathrm{o}$ & object & $\begin{array}{l}\text { MANN _ HEXE IX } \mathbf{X}_{\mathbf{L}} \text { FLIRT. HEXE TANZPARTNER SUCH. } \\
\text { MAN _ WITCH IX }{ }_{\text {L FLIRT. WITCH DANCING PARTNER SEARCH. }}\end{array}$ \\
\hline $\mathrm{S} 15 \mathrm{n}$ & none & $\begin{array}{l}\text { MANN _ _ HEXE _ _ FLIRT. HEXE TANZPARTNER SUCH. } \\
\text { MAN_ WITCH__ FLIRT. WITCH DANCING PARTNER SEARCH. }\end{array}$ \\
\hline S16so & both & $\begin{array}{l}\text { OMA IX } \mathbf{X}_{\mathbf{R}} \text { ARZT IX } \mathbf{X}_{\mathbf{L}} \text { FLIRT. ARZT GESCHENK KLEIN ÖFF. } \\
\text { GRANDMA IX }_{R} \text { DOCTOR IX } \mathrm{X}_{\mathrm{L}} \text { FLIRT. DOCTOR PRESENT LITTLE OPEN. }\end{array}$ \\
\hline S16s & subject & 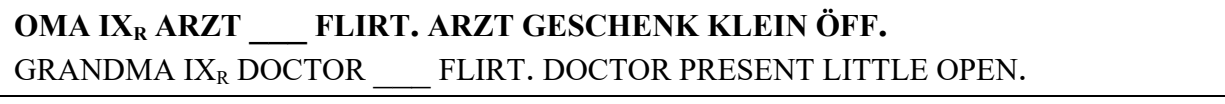 \\
\hline S16o & object & $\begin{array}{l}\text { OMA _ ARZT IX } \mathbf{X}_{\mathbf{L}} \text { FLIRT. ARZT GESCHENK KLEIN ÖFF. } \\
\text { GRANDMA _ DOCTOR IX }{ }_{L} \text { FLIRT. DOCTOR PRESENT LITTLE OPEN. }\end{array}$ \\
\hline S16n & none & $\begin{array}{l}\text { OMA __ ARZT _ FLIRT. ARZT GESCHENK KLEIN ÖFF. } \\
\text { GRANDMA__ DOCTOR__ FLIRT. DOCTOR PRESENT LITTLE OPEN. }\end{array}$ \\
\hline S17so & both & $\begin{array}{l}\text { KOCH IX } \mathbf{X}_{\mathbf{R}} \text { KELLNER IX } \mathbf{X}_{\mathbf{L}} \text { UNTERHALT. KELLNER VIEL GEHALT WÜNSCH. } \\
\text { COOK IX } \mathrm{X}_{\mathrm{R}} \text { WAITER IX } \mathrm{X}_{\mathrm{L}} \text { TALK. WAITER A-LOT SALARY WISH. }\end{array}$ \\
\hline $\mathrm{S} 17 \mathrm{~s}$ & subject & $\begin{array}{l}\text { KOCH IX } \mathbf{R}_{\mathbf{R}} \text { KELLNER } \\
\text { COOK IX UNTERHALT. KELLNER VIEL GEHALT WÜNSCH. }\end{array}$ \\
\hline S17o & object & $\begin{array}{l}\text { KOCH _ KELLNER IX } \mathbf{X}_{\mathbf{L}} \text { UNTERHALT. KELLNER VIEL GEHALT WÜNSCH. } \\
\text { COOK _ WAITER IX } \mathrm{X}_{\text {L TALK. WAITER A-LOT SALARY WISH. }}\end{array}$ \\
\hline $\mathrm{S} 17 \mathrm{n}$ & none & $\begin{array}{l}\text { KOCH _ KELLNER _ UNTERHALT. KELLNER VIEL GEHALT WÜNSCH. } \\
\text { COOK__ WAITER_TALK. WAITER A-LOT SALARY WISH. }\end{array}$ \\
\hline S18so & both & $\begin{array}{l}\text { OMA IX }{ }_{\mathbf{R}} \text { DETEKTIV IX } \mathbf{L}_{\mathbf{L}} \text { UNTERHALT. DETEKTIV RESTAURANT ITALIENISCH } \\
\text { HASS. } \\
\text { GRANDMA IX } \mathrm{X}_{\mathrm{R}} \text { DETECTIVE IX } \mathrm{X}_{\mathrm{L}} \text { TALK. DETECTIVE RESTAURANT ITALIAN HATE. }\end{array}$ \\
\hline S18s & subject & $\begin{array}{l}\text { OMA IX } X_{\mathbf{R}} \text { DETEKTIV _ UNTERHALT. DETEKTIV RESTAURANT ITALIENISCH } \\
\text { HASS. } \\
\text { GRANDMA IX } X_{R} \text { DETECTIVE _ TALK. DETECTIVE RESTAURANT ITALIAN HATE. }\end{array}$ \\
\hline S18o & object & $\begin{array}{l}\text { OMA__ DETEKTIV IX } \mathbf{L}_{\text {L UNTERHALT. DETEKTIV RESTAURANT ITALIENISCH }} \\
\text { HASS. } \\
\text { GRANDMA__ DETECTIVE IX I LALK. DETECTIVE RESTAURANT ITALIAN HATE. }\end{array}$ \\
\hline S18n & none & $\begin{array}{l}\text { OMA__ DETEKTIV__ UNTERHALT. DETEKTIV RESTAURANT ITALIENISCH } \\
\text { HASS. } \\
\text { GRANDMA__ DETECTIVE__TALK. DETECTIVE RESTAURANT ITALIAN HATE. }\end{array}$ \\
\hline S19so & both & $\begin{array}{l}\text { PRIESTER IX } \text { K }_{\mathbf{R}} \text { KÖNIG IX } \mathbf{L}_{\mathbf{L}} \text { UNTERHALT. KÖNIG ENGELSFIGUR BEWUNDER. } \\
\text { PRIEST IX } \mathrm{X}_{\mathrm{R}} \text { KING IX } \mathrm{X}_{\mathrm{L}} \text { TALK. KING ANGEL FIGURE ADMIRE. }\end{array}$ \\
\hline
\end{tabular}




\begin{tabular}{|c|c|c|}
\hline S19s & subject & $\begin{array}{l}\text { PRIESTER IX } \mathbf{X}_{\mathbf{R}} \text { KÖNIG _ } \_ \text {UNTERHALT. KÖNIG ENGELSFIGUR BEWUNDER. } \\
\text { PRIEST IX } \mathrm{I}_{\mathrm{R}} \mathrm{KING} \_ \text {TALK. KING ANGEL FIGURE ADMIRE. }\end{array}$ \\
\hline S19o & object & $\begin{array}{l}\text { PRIESTER } \\
\text { PRIEST } \_ \text {KÖNIG IX } \text { I }_{\mathbf{L}} \text { UNTERHALT. KÖNIG ENGELSFIGUR BEWUNDER. } \\
\end{array}$ \\
\hline S19n & none & $\begin{array}{l}\text { PRIESTER } \_ \text {KÖNIG _ UNTERHALT. KÖNIG ENGELSFIGUR BEWUNDER. } \\
\text { PRIEST _ KING__TALK. KING ANGEL FIGURE ADMIRE. }\end{array}$ \\
\hline S20so & both & $\begin{array}{l}\text { JÄGER IX }_{\mathbf{R}} \text { MANN IX } \mathbf{L}_{\mathbf{L}} \text { UNTERHALT. MANN AUTO GROß MAG(LIEB). } \\
\text { HUNTER IX }_{\text {R }} \text { MAN IX } \mathrm{X}_{L} \text { TALK. MAN CAR BIG LOVE. }\end{array}$ \\
\hline S20s & subject & $\begin{array}{l}\text { JÄGER IX } \mathbf{R}_{\mathbf{R}} \text { MANN_— UNTERHALT. MANN AUTO GROß MAG(LIEB). } \\
\text { HUNTER IX } X_{R} \text { MAN_ TALK. MAN CAR BIG LOVE. }\end{array}$ \\
\hline $\mathrm{S} 20 \mathrm{o}$ & object & $\begin{array}{l}\text { JÄGER__ MANN IX } \mathbf{X}_{\mathbf{L}} \text { UNTERHALT. MANN AUTO GROß MAG(LIEB). } \\
\text { HUNTER_ MAN IX }{ }_{\text {L TALK. MAN CAR BIG LOVE. }}\end{array}$ \\
\hline S20n & none & $\begin{array}{l}\text { JÄGER_ MANN__ UNTERHALT. MANN AUTO GROB MAG(LIEB). } \\
\text { HUNTER_ MAN__ TALK. MAN CAR BIG LOVE. }\end{array}$ \\
\hline S21so & both & $\begin{array}{l}\text { PRIESTER IX } \text { R NONNE IX }_{L} \text { DISKUTIER. NONNE REGELN NEU KRITISIER. } \\
\text { PRIEST IX } X_{R} \text { NUN IX } X_{L} \text { DISCUSS. NUN RULES NEW CRITICIZE. }\end{array}$ \\
\hline $\mathrm{S} 21 \mathrm{~s}$ & subject & $\begin{array}{l}\text { PRIESTER IX }_{R} \text { NONNE } \\
\text { PRIEST IX } X_{R} \text { NUN__ DISKUTIER. NONNE REGELN NEU KRITISIER. }\end{array}$ \\
\hline S21o & object & $\begin{array}{l}\text { PRIESTER _ NONNE IX } \text { IL }_{\text {LISKUTIER. NONNE REGELN NEU KRITISIER. }} \\
\text { PRIEST _ NUN IX } X_{L} \text { DISCUSS. NUN RULES NEW CRITICIZE. }\end{array}$ \\
\hline $\mathrm{S} 21 \mathrm{n}$ & none & $\begin{array}{l}\text { PRIESTER } \\
\text { PRIEST NONUNE }\end{array}$ \\
\hline S22so & both & $\begin{array}{l}\text { OPA IX } \mathbf{X}_{\mathbf{R}} \text { KELLNER IX } \mathbf{L}_{\mathrm{L}} \text { DISKUTIER. KELLNER GENAUE INFORMATIONEN } \\
\text { BRAUCH. } \\
\text { GRANDPA IX } \mathrm{X}_{\mathrm{R}} \text { WAITER IX } \mathrm{L}_{\mathrm{L}} \text { DISCUSS. WAITER EXACT INFORMATION NEED. } \\
\end{array}$ \\
\hline S22s & subject & 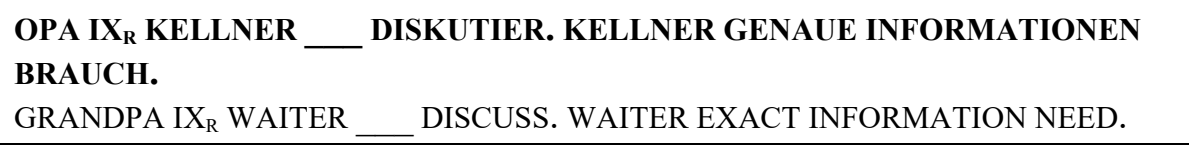 \\
\hline S22o & object & $\begin{array}{l}\text { OPA_ KELLNER IX }{ }_{\text {L }} \text { DISKUTIER. KELLNER GENAUE INFORMATIONEN } \\
\text { BRAUCH. } \\
\text { GRANDPA__ WAITER IX }{ }_{L} \text { DISCUSS. WAITER EXACT INFORMATION NEED. }\end{array}$ \\
\hline $\mathrm{S} 22 \mathrm{n}$ & none & $\begin{array}{l}\text { OPA_KELLNER__ DISKUTIER. KELLNER GENAUE INFORMATIONEN } \\
\text { BRAUCH. } \\
\text { GRANDPA__ WAITER _ DISCUSS. WAITER EXACT INFORMATION NEED. }\end{array}$ \\
\hline S23so & both & $\begin{array}{l}\text { HEXE IX } \mathbf{X}_{\mathbf{R}} \text { PIRAT IX } \mathbf{X}_{\mathbf{L}} \text { DISKUTIER. PIRAT ERSTEN PREIS GEWINN. } \\
\text { WITCH IXR }_{\text {PIRATE IX }} \text { DISCUSS. PIRATE FIRST PRICE WIN. }\end{array}$ \\
\hline $\mathrm{S} 23 \mathrm{~s}$ & subject & 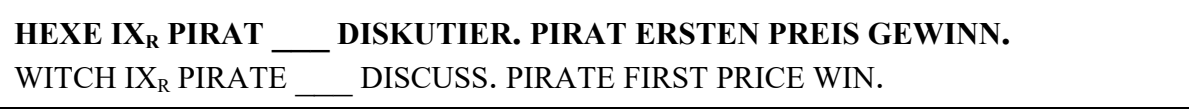 \\
\hline S23o & object & $\begin{array}{l}\text { HEXE _ PIRAT IX } \mathbf{L}_{\mathrm{L}} \text { DISKUTIER. PIRAT ERSTEN PREIS GEWINN. } \\
\text { WITCH _ PIRATE IX } \mathrm{X}_{\mathrm{L}} \text { DISCUSS. PIRATE FIRST PRICE WIN. }\end{array}$ \\
\hline $\mathrm{S} 23 \mathrm{n}$ & none & $\begin{array}{l}\text { HEXE __ PIRAT __ DISKUTIER. PIRAT ERSTEN PREIS GEWINN. } \\
\text { WITCH_ PIRATE__DISCUSS. PIRATE FIRST PRICE WIN. }\end{array}$ \\
\hline S24so & both & $\begin{array}{l}\text { KÖNIG IX } \mathbf{X}_{\mathbf{R}} \text { KOCH IX } \mathbf{X}_{\mathbf{L}} \text { DISKUTIER. KOCH FEIER ABLAUF VORSCHLAG. } \\
\text { KING IX }{ }_{R} \text { COOK IX }{ }_{L} \text { DISCUSS. COOK PARTY PROCEDURE SUGGEST. }\end{array}$ \\
\hline S24s & subject & 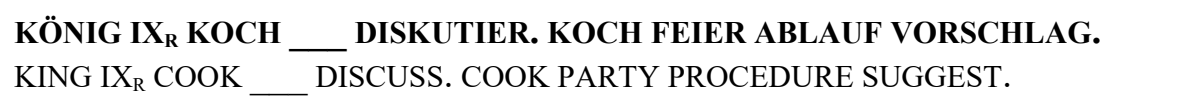 \\
\hline
\end{tabular}




\begin{tabular}{|c|c|c|}
\hline $\mathrm{S} 24 \mathrm{o}$ & object & $\begin{array}{l}\text { KÖNIG __ KOCH IX } \mathbf{X}_{\mathbf{L}} \text { DISKUTIER. KOCH FEIER ABLAUF VORSCHLAG. } \\
\text { KING _COOK IX }{ }_{\text {L }} \text { DISCUSS. COOK PARTY PROCEDURE SUGGEST. }\end{array}$ \\
\hline $\mathrm{S} 24 \mathrm{n}$ & none & $\begin{array}{l}\text { KÖNIG IX }{ }_{\mathrm{R}} \text { KOCH IX } \mathbf{L}_{\mathbf{L}} \text { DISKUTIER. KOCH FEIER ABLAUF VORSCHLAG. } \\
\text { KING _COOK__ DISCUSS. COOK PARTY PROCEDURE SUGGEST. }\end{array}$ \\
\hline $\mathrm{S} 25 \mathrm{so}$ & both & $\begin{array}{l}\text { CLOWN IX } \mathbf{X}_{\mathrm{R}} \text { ARZT IX } \mathrm{X}_{\mathbf{L}} \text { BEGEG. ARZT NEUIGKEIT GUT ERZÄHL. } \\
\text { CLOWN IX } \mathrm{X}_{\mathrm{R}} \text { DOCTOR IX } \mathrm{X}_{L} \text { ENCOUNTER. DOCTOR NEWS GOOD TELL. }\end{array}$ \\
\hline $\mathrm{S} 25 \mathrm{~s}$ & subject & $\begin{array}{ll}\text { CLOWN IX } & \text { ARZT } \\
\text { CLOWN IX } & \text { BEGEG. ARZT NEUIGKEIT GUT ERZÄHL. } \\
\text { DOCTOR _ } \quad \text { ENCOUNTER. DOCTOR NEWS GOOD TELL. }\end{array}$ \\
\hline $\mathrm{S} 250$ & object & $\begin{array}{l}\text { CLOWN — ARZT IX } \mathbf{L}_{\text {L BEGEG. ARZT NEUIGKEIT GUT ERZÄHL. }} \\
\text { CLOWN — DOCTOR IX } X_{L} \text { ENCOUNTER. DOCTOR NEWS GOOD TELL. }\end{array}$ \\
\hline $\mathrm{S} 25 \mathrm{n}$ & none & $\begin{array}{l}\text { CLOWN _ ARZT _ _ BEGEG. ARZT NEUIGKEIT GUT ERZÄHL. } \\
\text { CLOWN _ DOCTOR__ENCOUNTER. DOCTOR NEWS GOOD TELL. }\end{array}$ \\
\hline $\mathrm{S} 26 \mathrm{so}$ & both & $\begin{array}{l}\text { PILOT IX } \mathbf{X}_{\mathbf{R}} \text { OPA IX } \mathbf{X}_{\mathbf{L}} \text { BEGEG. OPA SCHACHSPIEL KONZENTRIER. } \\
\text { PILOT IX } \mathrm{X}_{\mathrm{R}} \text { GRANDPA IX } \mathrm{X}_{\mathrm{L}} \text { ENCOUNTER. GRANDPA CHESS GAME CONCENTRATE. }\end{array}$ \\
\hline S26s & subject & $\begin{array}{l}\text { PILOT IX } \mathbf{X}_{\mathbf{R}} \text { OPA } \_ \text {BEGEG. OPA SCHACHSPIEL KONZENTRIER. } \\
\text { PILOT IX }{ }_{R} \text { GRANDPA _ ENCOUNTER. GRANDPA CHESS GAME CONCENTRATE. }\end{array}$ \\
\hline S26o & object & $\begin{array}{l}\text { PILOT — OPA IX } \text { L }_{\text {LEGEG. OPA SCHACHSPIEL KONZENTRIER. }} \\
\text { PILOT_ GRANDPA IX I ENCOUNTER. GRANDPA CHESS GAME CONCENTRATE. }\end{array}$ \\
\hline S26n & none & $\begin{array}{l}\text { PILOT _ OPA _ _ BEGEG. OPA SCHACHSPIEL KONZENTRIER. } \\
\text { PILOT _ GRANDPA _ ENCOUNTER. GRANDPA CHESS GAME CONCENTRATE. }\end{array}$ \\
\hline S27so & both & $\begin{array}{l}\text { MALER IX } \mathbf{X}_{\mathbf{R}} \text { FRAU IX } \mathbf{X}_{\mathbf{L}} \text { BEGEG. FRAU WANDFARBE AUSSUCH. } \\
\text { PAINTER IX } X_{R} \text { WOMAN IX } X_{L} \text { ENCOUNTER. WOMAN WALL COLOR CHOOSE. }\end{array}$ \\
\hline $\mathrm{S} 27 \mathrm{~s}$ & subject & $\begin{array}{l}\text { MALER IX } \mathbf{X}_{\mathbf{R}} \text { FRAU __ BEGEG. FRAU WANDFARBE AUSSUCH. } \\
\text { PAINTER IX }{ }_{\mathrm{R}} \text { WOMAN _ ENCOUNTER. WOMAN WALL COLOR CHOOSE. }\end{array}$ \\
\hline $\mathrm{S} 27 \mathrm{o}$ & object & $\begin{array}{l}\text { MALER _ FRAU IX } \mathbf{X}_{\mathbf{L}} \text { BEGEG. FRAU WANDFARBE AUSSUCH. } \\
\text { PAINTER — WOMAN IX } \mathrm{X}_{\text {L }} \text { ENCOUNTER. WOMAN WALL COLOR CHOOSE. }\end{array}$ \\
\hline $\mathrm{S} 27 \mathrm{n}$ & none & $\begin{array}{l}\text { MALER __ FRAU _ _ BEGEG. FRAU WANDFARBE AUSSUCH. } \\
\text { PAINTER _ WOMAN__ ENCOUNTER. WOMAN WALL COLOR CHOOSE. }\end{array}$ \\
\hline S28so & both & $\begin{array}{l}\text { KOCH IX } \mathbf{X}_{\mathbf{R}} \text { PLOIZIST IX } \mathbf{X}_{\mathbf{L}} \text { BEGEG. POLIZIST DORF ALT ARBEIT. } \\
\text { COOK IX } \mathrm{X}_{\mathrm{R}} \text { POLICEMAN IX } \mathrm{X}_{\mathrm{L}} \text { ENCOUNTER. POLICEMAN VILLAGE OLD WORK. }\end{array}$ \\
\hline S28s & subject & 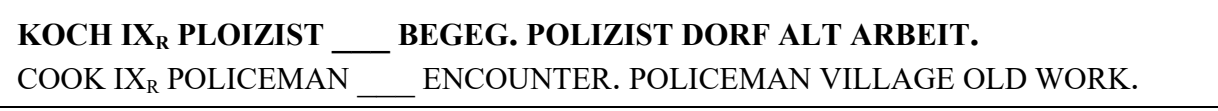 \\
\hline $\mathrm{S} 280$ & object & $\begin{array}{l}\text { KOCH _ PLOIZIST IX } \mathbf{L}_{\mathbf{L}} \text { BEGEG. POLIZIST DORF ALT ARBEIT. } \\
\text { COOK_ POLICEMAN IX } X_{L} \text { ENCOUNTER. POLICEMAN VILLAGE OLD WORK. }\end{array}$ \\
\hline $\mathrm{S} 28 \mathrm{n}$ & none & $\begin{array}{l}\text { KOCH _ PLOIZIST __ BEGEG. POLIZIST DORF ALT ARBEIT. } \\
\text { COOK__ POLICEMAN__ENCOUNTER. POLICEMAN VILLAGE OLD WORK. }\end{array}$ \\
\hline S29so & both & $\begin{array}{l}\text { POLIZIST IX } \mathbf{R}_{\mathbf{R}} \text { DIEB IX } \mathbf{X}_{\mathbf{L}} \text { VERFOLG. DIEB GUTE AUSDAUER DA. } \\
\text { POLICEMAN IX } \mathrm{X}_{\mathrm{R}} \text { THIEF IX } \mathrm{L}_{\mathrm{L}} \text { FOLLOW. THIEF GOOD ENDURANCE HAVE. }\end{array}$ \\
\hline S29s & subject & $\begin{array}{l}\text { POLIZIST IX } \text { R }_{\mathrm{R}} \text { DIEB __ VERFOLG. DIEB GUTE AUSDAUER DA. } \\
\text { POLICEMAN IX } X_{\mathrm{R}} \text { THIEF_ FOLLOW. THIEF GOOD ENDURANCE HAVE. }\end{array}$ \\
\hline $\mathrm{S} 290$ & object & $\begin{array}{l}\text { POLIZIST __ DIEB IX } \mathbf{X}_{\mathbf{L}} \text { VERFOLG. DIEB GUTE AUSDAUER DA. } \\
\text { POLICEMAN_ THIEF IX } X_{\text {L FOLLOW. THIEF GOOD ENDURANCE HAVE. }}\end{array}$ \\
\hline S29n & none & $\begin{array}{l}\text { POLIZIST __ DIEB _ VERFOLG. DIEB GUTE AUSDAUER DA. } \\
\text { POLICEMAN__ THIEF _ FOLLOW. THIEF GOOD ENDURANCE HAVE. }\end{array}$ \\
\hline S30so & both & $\begin{array}{l}\text { MÄDCHEN IX }{ }_{R} \text { COWBOY IX } X_{L} \text { VERFOLG. COWBOY BAUMSTAMM STOLPER. } \\
\text { GIRL IX } X_{R} \text { COWBOY IX } X_{L} \text { FOLLOW. COWBOY TREE TRUNK STUMBLE. }\end{array}$ \\
\hline
\end{tabular}




\begin{tabular}{|c|c|c|}
\hline S30s & subject & $\begin{array}{l}\text { MÄDCHEN IX }{ }_{R} \text { COWBOY _ VERFOLG. COWBOY BAUMSTAMM STOLPER. } \\
\text { GIRL IX } X_{R} \text { COWBOY___FOLLOW. COWBOY TREE TRUNK STUMBLE. }\end{array}$ \\
\hline $\mathrm{S} 30 \mathrm{o}$ & object & $\begin{array}{l}\text { MÄDCHEN _ COWBOY IX } \mathbf{L}_{\mathbf{L}} \text { VERFOLG. COWBOY BAUMSTAMM STOLPER. } \\
\text { GIRL__ COWBOY IX }{ }_{L} \text { FOLLOW. COWBOY TREE TRUNK STUMBLE. }\end{array}$ \\
\hline S30n & none & $\begin{array}{l}\text { MÄDCHEN _ COWBOY __ VERFOLG. COWBOY BAUMSTAMM STOLPER. } \\
\text { GIRL__COWBOY_ FOLLOW. COWBOY TREE TRUNK STUMBLE. }\end{array}$ \\
\hline S31so & both & 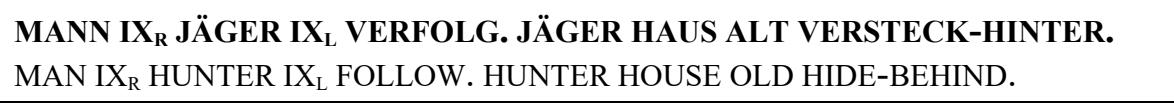 \\
\hline $\mathrm{S} 31 \mathrm{~s}$ & subject & $\begin{array}{l}\text { MANN IX } \mathbf{X}_{\mathbf{R}} \text { JÄGER _ VERFOLG. JÄGER HAUS ALT VERSTECK-HINTER. } \\
\text { MAN IX }{ }_{\text {R }} \text { HUNTER _ FOLLOW. HUNTER HOUSE OLD HIDE-BEHIND. }\end{array}$ \\
\hline S31o & object & $\begin{array}{l}\text { MANN__ JÄGER IX } \mathbf{X}_{\mathbf{L}} \text { VERFOLG. JÄGER HAUS ALT VERSTECK-HINTER. } \\
\text { MAN__ HUNTER IX }{ }_{\text {L }} \text { FOLLOW. HUNTER HOUSE OLD HIDE-BEHIND. }\end{array}$ \\
\hline $\mathrm{S} 31 \mathrm{n}$ & none & $\begin{array}{l}\text { MANN _ JÄGER _ VERFOLG. JÄGER HAUS ALT VERSTECK-HINTER. } \\
\text { MAN__ HUNTER_ FOLLOW. HUNTER HOUSE OLD HIDE-BEHIND. }\end{array}$ \\
\hline S32so & both & $\begin{array}{l}\text { PIRAT IX } \mathbf{X}_{\mathbf{R}} \text { JUNGE IX }_{\mathbf{L}} \text { VERFOLG. JUNGE TUNNEL DUNKELDURCHQUER. } \\
\text { PIRATE IX } \mathrm{X}_{\mathrm{R}} \text { BOY IX } \mathrm{I}_{\mathrm{L}} \text { FOLLOW. JUNGE TUNNEL DARK RUN-THROUGH. }\end{array}$ \\
\hline S32s & subject & $\begin{array}{l}\text { PIRAT IX }_{\mathbf{R}} \text { JUNGE _ VERFOLG. JUNGE TUNNEL DUNKELDURCHQUER. } \\
\text { PIRATE IX } \mathrm{X}_{\mathrm{R}} \text { BOY } \_ \text {FOLLOW. JUNGE TUNNEL DARK RUN-THROUGH. }\end{array}$ \\
\hline S32o & object & 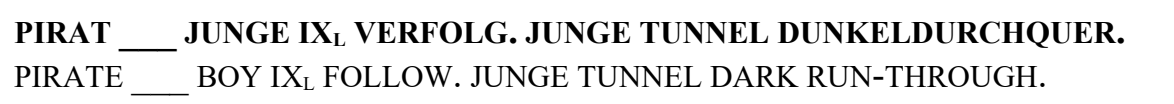 \\
\hline S32n & none & $\begin{array}{l}\text { PIRAT___JUNGE _ VERFOLG. JUNGE TUNNEL DUNKELDURCHQUER. } \\
\text { PIRATE__ BOY_FOLLOW. JUNGE TUNNEL DARK RUN-THROUGH. }\end{array}$ \\
\hline S33so & both & 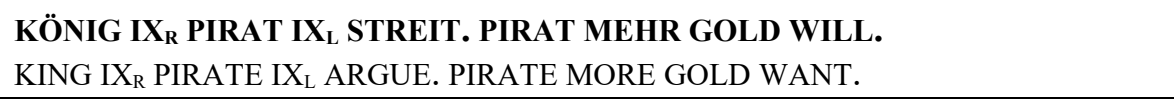 \\
\hline S33s & subject & $\begin{array}{l}\text { KÖNIG IX } \mathbf{X}_{\mathbf{R}} \text { PIRAT __ STREIT. PIRAT MEHR GOLD WILL. } \\
\text { KING IX }_{\text {R PIRATE__ ARGUE. PIRATE MORE GOLD WANT. }}\end{array}$ \\
\hline S33o & object & $\begin{array}{l}\text { KÖNIG __ PIRAT IX } \mathbf{X}_{\mathrm{L}} \text { STREIT. PIRAT MEHR GOLD WILL. } \\
\text { KING___PIRATE IX ARGUE. PIRATE MORE GOLD WANT. }\end{array}$ \\
\hline S33n & none & $\begin{array}{l}\text { KÖNIG___PIRAT __ STREIT. PIRAT MEHR GOLD WILL. } \\
\text { KING__PIRATE__ ARGUE. PIRATE MORE GOLD WANT. }\end{array}$ \\
\hline S34so & both & $\begin{array}{l}\text { LEHRER IX }_{\mathbf{R}} \text { JUNGE IX } \mathbf{X}_{\mathbf{L}} \text { STREIT. JUNGE THEMA SCHLECHT ERKLÄR. } \\
\text { TEACHER IX }_{R} \text { BOY IX } \mathrm{X}_{L} \text { ARGUE. BOY TOPIC BAD EXPLAIN. }\end{array}$ \\
\hline S34s & subject & $\begin{array}{l}\text { LEHRER IX } \mathbf{R}_{\mathbf{R}} \text { JUNGE _ } \text { STREIT. JUNGE THEMA SCHLECHT ERKLÄR. } \\
\text { TEACHER IX } X_{\mathrm{R}} \text { BOY _ ARGUE. BOY TOPIC BAD EXPLAIN. }\end{array}$ \\
\hline S34o & object & $\begin{array}{l}\text { LEHRER__ JUNGE IX } \text { L }_{\text {L STREIT. JUNGE THEMA SCHLECHT ERKLÄR. }} \text { _ BOY IX ARGUE. BOY TOPIC BAD EXPLAIN. } \\
\text { TEACHER _ B }\end{array}$ \\
\hline S34n & none & $\begin{array}{l}\text { LEHRER__JUNGE _ STREIT. JUNGE THEMA SCHLECHT ERKLÄR. } \\
\text { TEACHER__BOY_ARGUE. BOY TOPIC BAD EXPLAIN. }\end{array}$ \\
\hline S35so & both & $\begin{array}{l}\text { COWBOY IX } \mathbf{X}_{\mathbf{R}} \text { OPA IX } \mathbf{X}_{\mathbf{L}} \text { STREIT. OPA KARTENSPIEL VERLIER. } \\
\text { COWBOY IX } \mathrm{X}_{\mathrm{R}} \text { GRANDPA IX } \mathrm{X}_{\mathrm{L}} \text { ARGE. GRANDPA CARD GAME LOOSE. }\end{array}$ \\
\hline S35s & subject & $\begin{array}{l}\text { COWBOY IX } \mathbf{R}_{\mathbf{R}} \text { OPA } \_ \text {STREIT. OPA KARTENSPIEL VERLIER. } \\
\text { COWBOY IX } \mathrm{X}_{\mathrm{R}} \text { GRANDPA _ ARGUE. GRANDPA CARD GAME LOOSE. }\end{array}$ \\
\hline S35o & object & $\begin{array}{l}\text { COWBOY __ OPA IX } \mathrm{IX}_{\mathrm{L}} \text { STREIT. OPA KARTENSPIEL VERLIER. } \\
\text { COWBOY _ GRANDPA IX } \mathrm{X}_{\mathrm{L}} \text { ARGUE. GRANDPA CARD GAME LOOSE. }\end{array}$ \\
\hline
\end{tabular}




\begin{tabular}{|c|c|c|}
\hline S35n & none & $\begin{array}{l}\text { COWBOY __ OPA _ STREIT. OPA KARTENSPIEL VERLIER. } \\
\text { COWBOY _ GRANDPA _ ARGUE. GRANDPA CARD GAME LOOSE. }\end{array}$ \\
\hline S36so & both & $\begin{array}{l}\text { KRANKENSCHWESTER IX }{ }_{\mathbf{R}} \text { MÄDCHEN IX } \mathbf{X}_{\text {L STREIT. MÄDCHEN TABLETTEN }} \\
\text { WICHTIG VERGESS. } \\
\text { NURSE IX }{ }_{R} \text { GIRL IX }{ }_{L} \text { ARGUE. GIRL PILLS IMPORTANT FORGET. }\end{array}$ \\
\hline S36s & subject & $\begin{array}{l}\text { KRANKENSCHWESTER IX } X_{R} \text { MÄDCHEN _ _ STREIT. MÄDCHEN TABLETTEN } \\
\text { WICHTIG VERGESS. } \\
\text { NURSE IX }{ }_{R} \text { GIRL__ ARGUE. GIRL PILLS IMPORTANT FORGET. }\end{array}$ \\
\hline S36o & object & $\begin{array}{l}\text { KRANKENSCHWESTER _ MÄDCHEN IX } X_{\text {L }} \text { STREIT. MÄDCHEN TABLETTEN } \\
\text { WICHTIG VERGESS. } \\
\text { NURSE__ GIRL IX } X_{L} \text { ARGUE. GIRL PILLS IMPORTANT FORGET. }\end{array}$ \\
\hline S36n & none & $\begin{array}{l}\text { KRANKENSCHWESTER _ MÄDCHEN__ STREIT. MÄDCHEN TABLETTEN } \\
\text { WICHTIG VERGESS. } \\
\text { NURSE_ GIRL__ARGUE. GIRL PILLS IMPORTANT FORGET. }\end{array}$ \\
\hline $\mathrm{S} 37 \mathrm{so}$ & both & $\begin{array}{l}\text { COWBOY IX }_{R} \text { INDIANER IX }_{\text {L }} \text { PRÜGEL. INDIANER PFERD KRANK TÖT. } \\
\text { COWBOY IX } X_{R} \text { AMERINDIAN IX }{ }_{L} \text { BEAT. AMERINDIAN HORSE SICK KILL. }\end{array}$ \\
\hline S37s & subject & 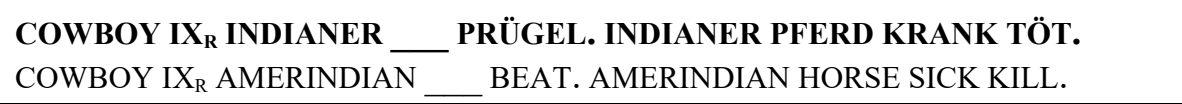 \\
\hline S37o & object & $\begin{array}{l}\text { COWBOY — INDIANER IX } \text { I }_{\mathrm{L}} \text { PRÜGEL. INDIANER PFERD KRANK TÖT. } \\
\text { COWBOY _ AMERINDIAN IX } X_{\mathrm{L}} \text { BEAT. AMERINDIAN HORSE SICK KILL. }\end{array}$ \\
\hline S37n & none & $\begin{array}{l}\text { COWBOY __ INDIANER _ PRÜGEL. INDIANER PFERD KRANK TÖT. } \\
\text { COWBOY__ AMERINDIAN__ BEAT. AMERINDIAN HORSE SICK KILL. }\end{array}$ \\
\hline S38so & both & $\begin{array}{l}\text { DIEB IX }_{\mathbf{R}} \text { DETEKTIV IX } \mathbf{L}_{\mathbf{L}} \text { PRÜGEL. DETEKTIV SCHNELL FLÜCHT WILL. } \\
\text { THIEF IX }_{R} \text { DETECTIVE IX } \mathrm{X}_{\mathrm{L}} \text { BEAT. DETECTIVE FAST ESCAPE WANT. }\end{array}$ \\
\hline S38s & subject & $\begin{array}{l}\text { DIEB IX } \mathbf{X}_{\mathbf{R}} \text { DETEKTIV__ PRÜGEL. DETEKTIV SCHNELL FLÜCHT WILL. } \\
\text { THIEF IX }_{\mathrm{R}} \text { DETECTIVE_ _ BEAT. DETECTIVE FAST ESCAPE WANT. }\end{array}$ \\
\hline S38o & object & $\begin{array}{l}\text { DIEB __ DETEKTIV IX } \text { L }_{\text {L }} \text { PRÜGEL. DETEKTIV SCHNELL FLÜCHT WILL. } \\
\text { THIEF__ DETECTIVE IX } \text { L }_{\text {L BEAT. DETECTIVE FAST ESCAPE WANT. }}\end{array}$ \\
\hline S38n & none & $\begin{array}{l}\text { DIEB__ DETEKTIV___ PRÜGEL. DETEKTIV SCHNELL FLÜCHT WILL. } \\
\text { THIEF__ DETECTIVE_ BEAT. DETECTIVE FAST ESCAPE WANT. }\end{array}$ \\
\hline S39so & both & $\begin{array}{l}\text { PIRAT IX } \mathbf{X}_{\mathbf{R}} \mathbf{J A ̈ G E R} \text { IX }_{\mathbf{L}} \text { PRÜGEL. JÄGER GUT VERTEIDIG KANN. } \\
\text { PIRATE IX } \mathrm{X}_{\mathrm{R}} \text { HUNTER IX } \mathrm{X}_{\mathrm{L}} \text { BEAT. HUNTER GOOD DEFEND CAN. }\end{array}$ \\
\hline S39s & subject & $\begin{array}{l}\text { PIRAT IX } \mathbf{X}_{\mathbf{R}} \text { JÄGER___ PRÜGEL. JÄGER GUT VERTEIDIG KANN. } \\
\text { PIRATE IX }{ }_{\mathrm{R}} \text { HUNTER__ BEAT. HUNTER GOOD DEFEND CAN. }\end{array}$ \\
\hline S39o & object & $\begin{array}{l}\text { PIRAT___ JÄGER IX } \mathbf{X}_{\mathbf{L}} \text { PRÜGEL. JÄGER GUT VERTEIDIG KANN. } \\
\text { PIRATE__ HUNTER IX } X_{\text {L BEAT. HUNTER GOOD DEFEND CAN. }}\end{array}$ \\
\hline S39n & none & $\begin{array}{l}\text { PIRAT___ JÄGER___PRÜGEL. JÄGER GUT VERTEIDIG KANN. } \\
\text { PIRATE_ _ HUNTER__ BEAT. HUNTER GOOD DEFEND CAN. }\end{array}$ \\
\hline S40so & both & $\begin{array}{l}\text { POLIZIST IX }{ }_{R} \text { SOLDAT IX } \text { PRÜGEL. SOLDAT GLASTÜR FALLEN-DURCH. }_{\text {POLICEMAN IX }} \text { SOLDIER IX } X_{L} \text { BEAT. SOLDIER GLASS DOOR FALL-THROUGH. } \\
\text { POLICEN }\end{array}$ \\
\hline $\mathrm{S} 40 \mathrm{~s}$ & subject & $\begin{array}{l}\text { POLIZIST IX } \text { R }_{\mathbf{R}} \text { SOLDAT __ PRÜGEL. SOLDAT GLASTÜR FALLEN-DURCH. } \\
\text { POLICEMAN IX } X_{R} \text { SOLDIER _ BEAT. SOLDIER GLASS DOOR FALL-THROUGH. }\end{array}$ \\
\hline S40o & object & $\begin{array}{l}\text { POLIZIST___SOLDAT IX } \mathbf{X}_{\text {L }} \text { PRÜGEL. SOLDAT GLASTÜR FALLEN-DURCH. } \\
\text { POLICEMAN___SOLDIER IX BEAT. SOLDIER GLASS DOOR FALL-THROUGH. }\end{array}$ \\
\hline S40n & none & 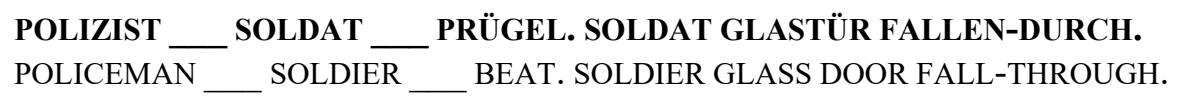 \\
\hline
\end{tabular}


Table 2: Stimulus material for sentences continuing with the subject

\begin{tabular}{|c|c|c|}
\hline $\begin{array}{l}\text { Video } \\
\text { name }\end{array}$ & $\begin{array}{l}\text { Condi } \\
\text {-tion } \\
\end{array}$ & Sentence set \\
\hline F01so & both & $\begin{array}{l}\text { HEXE IX } \mathbf{R}_{\mathbf{R}} \text { KOCH IX } \mathbf{L}_{\mathrm{L}} \text { KÜSS. HEXE STARK MUNDGERUCH DA. } \\
\text { WITCH IX }_{\mathrm{R}} \text { COOK IX } \mathrm{X}_{\mathrm{L}} \text { KISS. WITCH STRONG MOUTH-ODOR HAVE. }\end{array}$ \\
\hline $\mathrm{F} 01 \mathrm{~s}$ & subject & $\begin{array}{l}\text { HEXE IX } \mathbf{X}_{\mathbf{R}} \text { KOCH__ KÜSS. HEXE STARK MUNDGERUCH DA. } \\
\text { WITCH IX }_{\mathrm{R}} \text { COOK__ KISS. WITCH STRONG MOUTH-ODOR HAVE. }\end{array}$ \\
\hline F01o & object & $\begin{array}{l}\text { HEXE _ KOCH IX } \mathbf{X}_{\mathbf{L}} \text { KÜSS. HEXE STARK MUNDGERUCH DA. } \\
\text { WITCH _ COOK IX } \mathrm{X}_{\mathrm{L}} \text { KISS. WITCH STRONG MOUTH-ODOR HAVE. }\end{array}$ \\
\hline F01n & none & $\begin{array}{l}\text { HEXE _ KOCH _ KÜSS. HEXE STARK MUNDGERUCH DA. } \\
\text { WITCH_ COOK_ KISS. WITCH STRONG MOUTH-ODOR HAVE. }\end{array}$ \\
\hline F02so & both & $\begin{array}{l}\text { CLOWN IX } \mathbf{X}_{\mathbf{R}} \text { COYBOW IX } \mathbf{X}_{\mathbf{L}} \text { KÜSS. CLOWN SCHNELL ABHAU WILL. } \\
\text { CLOWN IX }{ }_{R} \text { COWBOY IX }{ }_{R} \text { KISS. CLOWN FAST RUN-AWAY WANT. }\end{array}$ \\
\hline F02s & subject & $\begin{array}{l}\text { CLOWN IX } \mathbf{X}_{\mathrm{R}} \text { COYBOW __ KÜSS. CLOWN SCHNELL ABHAU WILL. } \\
\text { CLOWN IX }{ }_{\mathrm{R}} \text { COWBOY_ KISS. CLOWN FAST RUN-AWAY WANT. }\end{array}$ \\
\hline F02o & object & 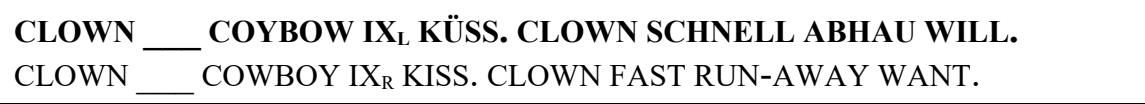 \\
\hline $\mathrm{F} 02 \mathrm{n}$ & none & $\begin{array}{l}\text { CLOWN _ COYBOW _ KÜSS. CLOWN SCHNELL ABHAU WILL. } \\
\text { CLOWN_ COWBOY _ KISS. CLOWN FAST RUN-AWAY WANT. }\end{array}$ \\
\hline F03so & both & $\begin{array}{l}\text { MALER IX } \mathbf{X}_{\mathbf{R}} \text { NONNE IX } \mathbf{X}_{L} \text { KÜSS. MALER NOCHMAL MACHEN WÜNSCH. } \\
\text { PAINTER IX }{ }_{R} \text { NUN IX }{ }_{L} \text { KISS. PAINTER AGAIN DO WANT. }\end{array}$ \\
\hline F03s & subject & $\begin{array}{l}\text { MALER IX }_{\mathbf{R}} \text { NONNE } \\
\text { PAINTER IX }{ }_{R} \text { NUN }\end{array}$ \\
\hline F03o & object & $\begin{array}{l}\text { MALER _ NONNE IX } \mathbf{X}_{\mathrm{L}} \text { KÜSS. MALER NOCHMAL MACHEN WÜNSCH. } \\
\text { PAINTER _ NUN IX } X_{L} \text { KISS. PAINTER AGAIN DO WANT. }\end{array}$ \\
\hline F03n & none & $\begin{array}{l}\text { MALER _ NONNE _ KÜSS. MALER NOCHMAL MACHEN WÜNSCH. } \\
\text { PAINTER_ NUN_KISS. PAINTER AGAIN DO WANT. }\end{array}$ \\
\hline F04so & both & $\begin{array}{l}\text { POLIZIST IX } \text { INDIANER IX }_{\mathbf{L}} \text { KÜSS. POLIZIST LIPPEN WEICH MAG. } \\
\text { POLICEMAN IX } \mathrm{X}_{\mathrm{R}} \text { AMERINDIAN IX } \mathrm{X}_{\mathrm{L}} \text { KISS. POLICEMAN LIPS SOFT LIKE. }\end{array}$ \\
\hline $\mathrm{F} 04 \mathrm{~s}$ & subject & $\begin{array}{l}\text { POLIZIST IX } \mathbf{R}_{\mathbf{R}} \text { INDIANER } \_ \text {KÜSS. POLIZIST LIPPEN WEICH MAG. } \\
\text { POLICEMAN IX } X_{R} \text { AMERINDIAN _ KISS. POLICEMAN LIPS SOFT LIKE. }\end{array}$ \\
\hline F04o & object & 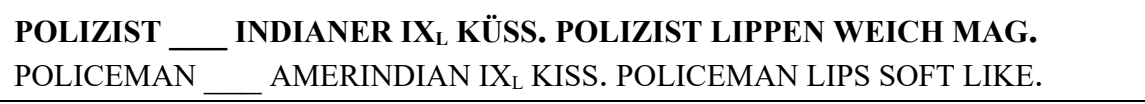 \\
\hline F04n & none & $\begin{array}{l}\text { POLIZIST _ INDIANER__ KÜSS. POLIZIST LIPPEN WEICH MAG. } \\
\text { POLICEMAN__ AMERINDIAN__ KISS. POLICEMAN LIPS SOFT LIKE. }\end{array}$ \\
\hline F05so & both & $\begin{array}{l}\text { KRANKENSCHESTER I } X_{R} \text { OPA I } X_{L} \text { TREFF. KRANKENSCHWESTER } \\
\text { INTERESSANTE UNTERHALTUNG WÜNSCH. } \\
\text { NURSE IX } X_{R} \text { GRANDPA IX } X_{L} \text { MEET. NURSE INTERSTING CONVERSATION WISH. }\end{array}$ \\
\hline $\mathrm{F} 05 \mathrm{~s}$ & subject & $\begin{array}{l}\text { KRANKENSCHESTER IX } X_{R} \text { OPA } \_ \text {TREFF. KRANKENSCHWESTER } \\
\text { INTERESSANTE UNTERHALTUNG WÜNSCH. } \\
\text { NURSE IX } X_{R} \text { GRANDPA__ MEET. NURSE INTERSTING CONVERSATION WISH. }\end{array}$ \\
\hline F05o & object & $\begin{array}{l}\text { KRANKENSCHESTER__ OPA IX } \mathrm{L}_{\mathrm{L}} \text { TREFF. KRANKENSCHWESTER } \\
\text { INTERESSANTE UNTERHALTUNG WÜNSCH. } \\
\text { NURSE__ GRANDPA IX }{ }_{L} \text { MEET. NURSE INTERSTING CONVERSATION WISH. }\end{array}$ \\
\hline
\end{tabular}




\begin{tabular}{|c|c|c|}
\hline F05n & none & $\begin{array}{l}\text { KRANKENSCHESTER } \_ \text {OPA } \_ \text {TREFF. KRANKENSCHWESTER } \\
\text { INTERESSANTE UNTERHALTUNG WÜNSCH. } \\
\text { NURSE _ GRANDPA_ MEET. NURSE INTERSTING CONVERSATION WISH. }\end{array}$ \\
\hline F06so & both & $\begin{array}{l}\text { MANN IX } \mathbf{X}_{\mathbf{R}} \text { DETEKTIV IX } \mathbf{X}_{\mathbf{L}} \text { TREFF. MANN WICHTIGE INFORMATIONEN SUCH. } \\
\text { MAN IX }_{R} \text { DETECTIVE IX }{ }_{L} \text { MEET. MAN IMPORTANT INFORMATION SEARCH. }\end{array}$ \\
\hline F06s & subject & $\begin{array}{l}\text { MANN IX } \mathbf{X}_{\mathbf{R}} \text { DETEKTIV — TREFF. MANN WICHTIGE INFORMATIONEN SUCH. } \\
\text { MAN IX }{ }_{\mathrm{R}} \text { DETECTIVE_ MEET. MAN IMPORTANT INFORMATION SEARCH. }\end{array}$ \\
\hline F06o & object & $\begin{array}{l}\text { MANN _ DETEKTIV IX } \mathbf{X}_{\mathbf{L}} \text { TREFF. MANN WICHTIGE INFORMATIONEN SUCH. } \\
\text { MAN } \_ \text {DETECTIVE IX } \mathrm{X}_{\mathrm{L}} \text { MEET. MAN IMPORTANT INFORMATION SEARCH. }\end{array}$ \\
\hline F06n & none & $\begin{array}{l}\text { MANN _ DETEKTIV _ _ TREFF. MANN WICHTIGE INFORMATIONEN SUCH. } \\
\text { MAN _ DETECTIVE__ MEET. MAN IMPORTANT INFORMATION SEARCH. }\end{array}$ \\
\hline F07so & both & $\begin{array}{l}\text { MÄDCHEN IX } \text { KÖNIG IX }_{\text {L }} \text { TREFF. MÄDCHEN SCHMUCK TEUER BESITZ. } \\
\text { GIRL IX }_{R} \text { KING IX }{ }_{L} \text { MEET. GIRL JEWELRY EXPENSIVE OWN. }\end{array}$ \\
\hline $\mathrm{F} 07 \mathrm{~s}$ & subject & $\begin{array}{l}\text { MÄDCHEN IX }{ }_{R} \text { KÖNIG__ TREFF. MÄDCHEN SCHMUCK TEUER BESITZ. } \\
\text { GIRL IX }_{R} \text { KING__ MEET. GIRL JEWELRY EXPENSIVE OWN. }\end{array}$ \\
\hline F07o & object & 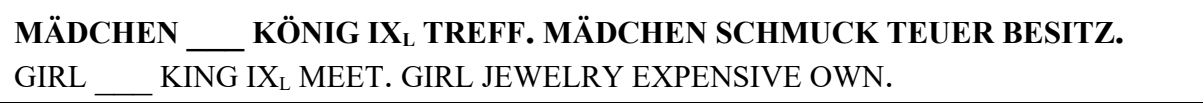 \\
\hline F07n & none & $\begin{array}{l}\text { MÄDCHEN__ KÖNIG__ TREFF. MÄDCHEN SCHMUCK TEUER BESITZ. } \\
\text { GIRL___KING__ MEET. GIRL JEWELRY EXPENSIVE OWN. }\end{array}$ \\
\hline F08so & both & $\begin{array}{l}\text { INDIANER IX } \mathbf{X}_{\mathbf{R}} \text { JUNGE IX }_{\mathbf{L}} \text { TREFF. INDIANER SPIEL SCHÖN ÜBERLEG. } \\
\text { AMERINDIAN IX } \mathrm{X}_{\mathrm{R}} \text { BOY IX } \mathrm{X}_{\mathrm{L}} \text { MEET. AMERINDIAN GAME NICE CONSTRUCT. }\end{array}$ \\
\hline F08s & subject & $\begin{array}{l}\text { INDIANER IX } \mathbf{X}_{\mathbf{R}} \text { JUNGE — TREFF. INDIANER SPIEL SCHÖN ÜBERLEG. } \\
\text { AMERINDIAN IX } \text { I }_{\mathrm{R}} \text { BOY } \\
\end{array}$ \\
\hline F08o & object & 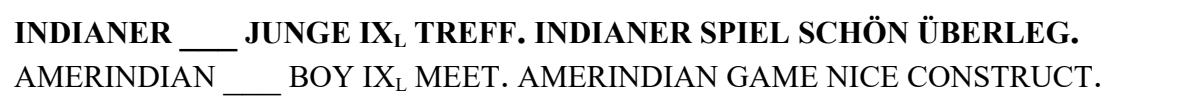 \\
\hline F08n & none & $\begin{array}{l}\begin{array}{l}\text { INDIANER _ JUNGE } \\
\text { AMERINDIAN }\end{array} \text { BOY TREFF. INDIANER SPIEL SCHÖN ÜBERLEG. } \\
\text { MEET. AMERINDIAN GAME NICE CONSTRUCT. }\end{array}$ \\
\hline F09so & both & $\begin{array}{l}\text { FRAU IX } \mathbf{X}_{\mathbf{R}} \mathbf{J A ̈ G E R} \text { IX }_{\mathbf{L}} \text { KENNENLERN. FRAU VIEL FLEISCH MAG(LIEB). } \\
\text { WOMAN IX } \mathrm{X}_{\mathrm{R}} \text { HUNTER IX } \mathrm{X}_{\mathrm{L}} \text { GET-TO-KNOW. WOMAN A-LOT MEAT LOVE. }\end{array}$ \\
\hline F09s & subject & $\begin{array}{l}\text { FRAU IX } \mathbf{X}_{\mathbf{R}} \text { JÄGER __ KENNENLERN. FRAU VIEL FLEISCH MAG(LIEB). } \\
\text { WOMAN IX } \mathrm{X}_{\mathrm{R}} \text { HUNTER __ GET-TO-KNOW. WOMAN A-LOT MEAT LOVE. }\end{array}$ \\
\hline F09o & object & $\begin{array}{l}\text { FRAU __ JÄGER IX } \text { I }_{\text {LENNENLERN. FRAU VIEL FLEISCH MAG(LIEB). }} \\
\text { WOMAN _ } \quad \text { HUNTER IX } \mathrm{X}_{\text {L }} \text { GET-TO-KNOW. WOMAN A-LOT MEAT LOVE. }\end{array}$ \\
\hline F09n & none & 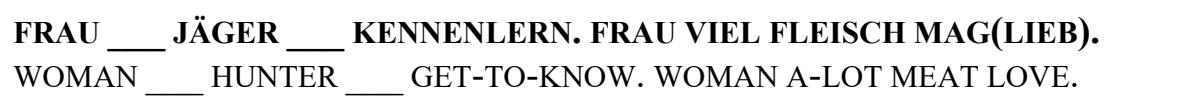 \\
\hline F10so & both & $\begin{array}{l}\text { COWBOY IX } \mathbf{X}_{\mathbf{R}} \text { OMA IX } \mathbf{X}_{\mathbf{L}} \text { KENNENLERN. COWBOY GESCHICHTEN ALT KENN. } \\
\text { COWBOY IX } \mathrm{X}_{\mathrm{R}} \text { GRANDMA IX } \mathrm{X}_{\mathrm{L}} \text { GET-TO-KNOW. COWBOY STORIES OLD KNOW. }\end{array}$ \\
\hline F10s & subject & $\begin{array}{l}\text { COWBOY IX } \mathbf{X}_{\mathbf{R}} \text { OMA } \_ \text {KENNENLERN. COWBOY GESCHICHTEN ALT KENN. } \\
\text { COWBOY IX } \mathrm{IX}_{\mathrm{R}} \text { GRANDMA _ GET-TO-KNOW. COWBOY STORIES OLD KNOW. }\end{array}$ \\
\hline F10o & object & 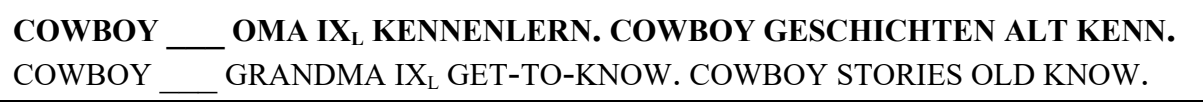 \\
\hline F10n & none & $\begin{array}{l}\text { COWBOY _ OMA __ KENNENLERN. COWBOY GESCHICHTEN ALT KENN. } \\
\text { COWBOY _ GRANDMA__ GET-TO-KNOW. COWBOY STORIES OLD KNOW. }\end{array}$ \\
\hline F11so & both & $\begin{array}{l}\text { DIEB IX }_{\text {R }} \text { HEXE IX } \mathbf{X}_{L} \text { KENNENLERN. DIEB ZUKUNFT WISSEN WILL. } \\
\text { THIEF IX } X_{R} \text { WITCH IX } I_{L} \text { GET-TO-KNOW. THIEF FUTURE KNOW WANT. }\end{array}$ \\
\hline
\end{tabular}




\begin{tabular}{|c|c|c|}
\hline F11s & subject & $\begin{array}{l}\text { DIEB IX } \text { R }_{\mathbf{R}} \text { HEXE __ KENNENLERN. DIEB ZUKUNFT WISSEN WILL. } \\
\text { THIEF IX }_{\mathrm{R}} \text { WITCH _ } \\
\end{array}$ \\
\hline F11o & object & $\begin{array}{l}\text { DIEB _ HEXE IX } \mathbf{X}_{\mathrm{L}} \text { KENNENLERN. DIEB ZUKUNFT WISSEN WILL. } \\
\text { THIEF_ WITCH IX } \mathrm{X}_{\text {L GET-TO-KNOW. THIEF FUTURE KNOW WANT. }} \text { _ W }\end{array}$ \\
\hline F11n & none & $\begin{array}{l}\text { DIEB __ HEXE __ KENNENLERN. DIEB ZUKUNFT WISSEN WILL. } \\
\text { THIEF_ WITCH__ GET-TO-KNOW. THIEF FUTURE KNOW WANT. }\end{array}$ \\
\hline F12so & both & $\begin{array}{l}\text { MANN IX }{ }_{\mathbf{R}} \text { OPA IX } \mathbf{L}_{\mathbf{L}} \text { KENNENLERN. MANN GLEICHES HOTEL BUCH. } \\
\text { MAN IX } \text { RRANDPA IX } \mathrm{X}_{\mathrm{L}} \text { GET-TO-KNOW. MAN SAME HOTEL BOOK. }\end{array}$ \\
\hline F12s & subject & $\begin{array}{l}\text { MANN IX } \mathbf{X}_{\mathbf{R}} \text { OPA } \_ \text {KENNENLERN. MANN GLEICHES HOTEL BUCH. } \\
\text { MAN IX }_{\mathrm{R}} \text { GRANDPA_ GET-TO-KNOW. MAN SAME HOTEL BOOK. }\end{array}$ \\
\hline F12o & object & $\begin{array}{l}\text { MANN _ } \text { OPA IX } \mathbf{X}_{\mathbf{L}} \text { KENNENLERN. MANN GLEICHES HOTEL BUCH. } \\
\text { MAN _ GRANDPA IX } \mathrm{X}_{\mathrm{L}} \text { GET-TO-KNOW. MAN SAME HOTEL BOOK. }\end{array}$ \\
\hline F12n & none & $\begin{array}{l}\text { MANN _ _ OPA___ KENNENLERN. MANN GLEICHES HOTEL BUCH. } \\
\text { MAN__ GRANDPA _ GET-TO-KNOW. MAN SAME HOTEL BOOK. }\end{array}$ \\
\hline F13so & both & $\begin{array}{l}\text { MÄDCHEN IX } \mathbf{X}_{\mathbf{R}} \text { MALER IX } \mathbf{X}_{\mathrm{L}} \text { FLIRT. MÄDCHEN VIEL HOFFNUNG DA. } \\
\text { GIRL IX } \mathrm{X}_{\mathrm{R}} \text { PAINTER IX } \mathrm{X}_{\mathrm{L}} \text { FLIRT. GIRL A-LOT HOPE HAVE. }\end{array}$ \\
\hline F13s & subject & 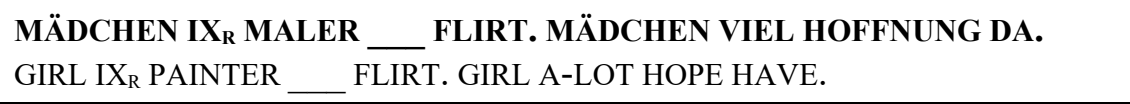 \\
\hline F13o & object & $\begin{array}{l}\text { MÄDCHEN _ MALER IX } \mathbf{X}_{\mathbf{L}} \text { FLIRT. MÄDCHEN VIEL HOFFNUNG DA. } \\
\text { GIRL__ PAINTER IX } X_{L} \text { FLIRT. GIRL A-LOT HOPE HAVE. }\end{array}$ \\
\hline F13n & none & $\begin{array}{l}\text { MÄDCHEN IX_ MALER__ FLIRT. MÄDCHEN VIEL HOFFNUNG DA. } \\
\text { GIRL__ PAINTER__ FLIRT. GIRL A-LOT HOPE HAVE. }\end{array}$ \\
\hline F14so & both & $\begin{array}{l}\text { JUNGE IX } \mathbf{R}_{\mathbf{R}} \text { DIEB IX } \mathbf{X}_{\mathbf{L}} \text { FLIRT. JUNGE SPANNED SITUATION MAG. } \\
\text { BOY IX } \mathrm{R}_{\mathrm{R}} \text { THIEF IX } \mathrm{X}_{\mathrm{L}} \text { FLIRT. BOY EXCITING SITUATION LIKE. }\end{array}$ \\
\hline F14s & subject & 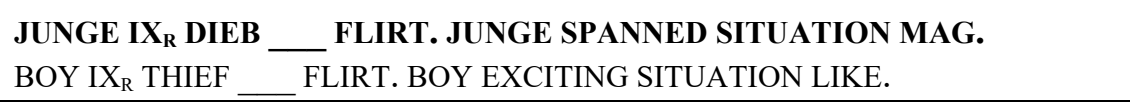 \\
\hline F14o & object & $\begin{array}{l}\text { JUNGE } \_ \text {DIEB IX } \mathbf{L}_{\text {L }} \text { FLIRT. JUNGE SPANNED SITUATION MAG. } \\
\text { BOY _ THIEF IX } \mathrm{L}_{\mathrm{L}} \text { FLIRT. BOY EXCITING SITUATION LIKE. }\end{array}$ \\
\hline $\mathrm{F} 14 \mathrm{n}$ & none & $\begin{array}{l}\text { JUNGE __ DIEB __ FLIRT. JUNGE SPANNED SITUATION MAG. } \\
\text { BOY _ _ THIEF_ FLIRT. BOY EXCITING SITUATION LIKE. }\end{array}$ \\
\hline F15so & both & $\begin{array}{l}\text { OPA IX } \mathbf{R}_{\mathbf{R}} \text { NONNE IX } \mathbf{X}_{\mathbf{L}} \text { FLIRT. OPA LANG ALLEIN GEWES. } \\
\text { GRANDPA IX } X_{R} \text { NUN IX } X_{L} \text { FLIRT. GRANDPA LONG ALONE WAS. }\end{array}$ \\
\hline F15s & subject & $\begin{array}{ll}\text { OPA IX }_{\mathbf{R}} \text { NONNE } & \text { FLIRT. OPA LANG ALLEIN GEWES. } \\
\text { GRANDPA IX } \mathrm{X}_{\mathrm{R}} \mathrm{NUN} \_ \text {FLIRT. GRANDPA LONG ALONE WAS. }\end{array}$ \\
\hline F15o & object & $\begin{array}{l}\text { OPA } \_ \text {NONNE IX } \mathbf{X}_{\mathbf{L}} \text { FLIRT. OPA LANG ALLEIN GEWES. } \\
\text { GRANDPA _ NUN IX } X_{\text {L FLIRT. GRANDPA LONG ALONE WAS. }}\end{array}$ \\
\hline F15n & none & $\begin{array}{l}\text { OPA __ NONNE } \_ \text {FLIRT. OPA LANG ALLEIN GEWES. } \\
\text { GRANDPA__ NUN _ FLIRT. GRANDPA LONG ALONE WAS. }\end{array}$ \\
\hline F16so & both & $\begin{array}{l}\text { PIRAT IX } \mathbf{X}_{\mathbf{R}} \text { INDIANER IX } \mathbf{X}_{\mathbf{L}} \text { FLIRT. PIRAT INTERESSE SCHNELL VERLIER. } \\
\text { PIRATE IX }_{\text {R }} \text { AMERINDIAN IX }{ }_{L} \text { FLIRT. PIRATE INTEREST FAST LOOSE. }\end{array}$ \\
\hline F16s & subject & 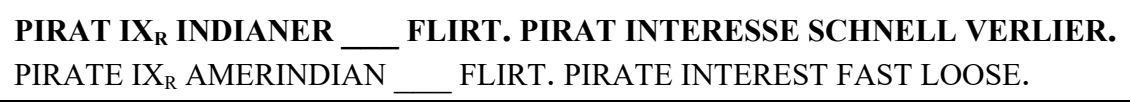 \\
\hline F16o & object & $\begin{array}{l}\text { PIRAT__ INDIANER IX } \text { I }_{\mathbf{L}} \text { FLIRT. PIRAT INTERESSE SCHNELL VERLIER. } \\
\text { PIRATE__ AMERINDIAN IX } \text { L }_{\text {L FLIRT. PIRATE INTEREST FAST LOOSE. }}\end{array}$ \\
\hline F16n & none & $\begin{array}{l}\text { PIRAT___ INDIANER _ } \_ \text {FLIRT. PIRAT INTERESSE SCHNELL VERLIER. } \\
\text { PIRATE__AMERINDIAN _ FLIRT. PIRATE INTEREST FAST LOOSE. }\end{array}$ \\
\hline
\end{tabular}




\begin{tabular}{|c|c|c|}
\hline F17so & both & $\begin{array}{l}\text { LEHRER IX }{ }_{\mathbf{R}} \text { MÄDCHEN IX } \mathbf{L}_{\mathbf{L}} \text { UNTERHALT. LEHRER ANDERE STADT GEBOR. } \\
\text { TEACHER IX } \mathrm{K}_{\mathrm{R}} \text { GIRL IX } \mathrm{X}_{\mathrm{L}} \text { TALK. TEACHER ANOTHER CITY BORN. }\end{array}$ \\
\hline F17s & subject & $\begin{array}{l}\text { LEHRER IX } \mathbf{X}_{\mathbf{R}} \text { MÄDCHEN } \_ \text {UNTERHALT. LEHRER ANDERE STADT GEBOR. } \\
\text { TEACHER IX }{ }_{R} \text { GIRL _ TALK. TEACHER ANOTHER CITY BORN. }\end{array}$ \\
\hline F17o & object & $\begin{array}{l}\text { LEHRER_—_ MÄDCHEN IX } \text { L }_{\text {LNTERHALT. LEHRER ANDERE STADT GEBOR. }} \\
\text { TEACHER _ GIRL IX } \mathrm{X}_{\text {L TALK. TEACHER ANOTHER CITY BORN. }}\end{array}$ \\
\hline F17n & none & $\begin{array}{l}\text { LEHRER _ MÄDCHEN _ _ UNTERHALT. LEHRER ANDERE STADT GEBOR. } \\
\text { TEACHER__ GIRL__ TALK. TEACHER ANOTHER CITY BORN. }\end{array}$ \\
\hline F18so & both & $\begin{array}{l}\text { POLIZIST IX } \mathbf{R}_{\mathbf{R}} \text { KRANKENSCHWESTER IX } \mathrm{X}_{\text {L }} \text { UNTERHALT. POLIZIST AUTO NEU } \\
\text { KAUF. } \\
\text { POLICEMAN IX } \mathrm{X}_{R} \text { NURSE IX }{ }_{R} \text { TALK. POLICEMAN CAR NEW BUY. }\end{array}$ \\
\hline F18s & subject & $\begin{array}{l}\text { POLIZIST IX } X_{R} \text { KRANKENSCHWESTER _ UNTERHALT. POLIZIST AUTO NEU } \\
\text { KAUF. } \\
\text { POLICEMAN IX }{ }_{R} \text { NURSE __ TALK. POLICEMAN CAR NEW BUY. }\end{array}$ \\
\hline F18o & object & $\begin{array}{l}\text { POLIZIST _ KRANKENSCHWESTER IX } \text { L }_{\text {L UNTERHALT. POLIZIST AUTO NEU }} \\
\text { KAUF. } \\
\text { POLICEMAN__ NURSE IX }{ }_{R} \text { TALK. POLICEMAN CAR NEW BUY. }\end{array}$ \\
\hline F18n & none & $\begin{array}{l}\text { POLIZIST _ KRANKENSCHWESTER _ _ UNTERHALT. POLIZIST AUTO NEU } \\
\text { KAUF. } \\
\text { POLICEMAN _ NURSE _ TALK. POLICEMAN CAR NEW BUY. }\end{array}$ \\
\hline F19so & both & 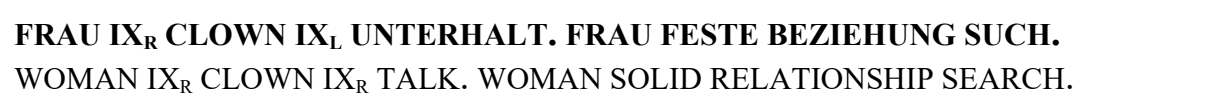 \\
\hline F19s & subject & $\begin{array}{l}\text { FRAU IX } \mathbf{R}_{\mathbf{R}} \text { CLOWN _ UNTERHALT. FRAU FESTE BEZIEHUNG SUCH. } \\
\text { WOMAN IX } \mathrm{X}_{\mathrm{R}} \text { CLOWN _ TALK. WOMAN SOLID RELATIONSHIP SEARCH. }\end{array}$ \\
\hline F19o & object & 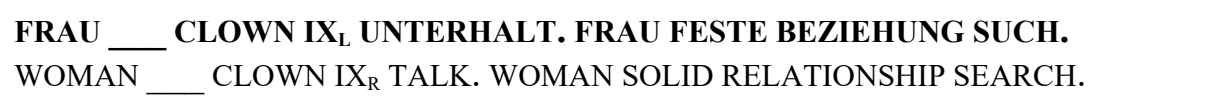 \\
\hline F19n & none & $\begin{array}{lll}\text { FRAU } & \text { CLOWN } \\
\text { WOMAN _ } & \text { CLOWN } \_ \text {UNTERHALT. FRAU FESTE BEZIEHUNG SUCH. } \\
\end{array}$ \\
\hline F20so & both & $\begin{array}{l}\text { SOLDAT IX } \mathbf{R}_{\mathbf{R}} \text { PILOT IX } \mathbf{X}_{\mathbf{L}} \text { UNTERHALT. SOLDAT SOMMERPARTY ORGANISIER. } \\
\text { SOLDIER IX } \mathrm{X}_{\mathrm{R}} \text { PILOT IX } \mathrm{X}_{\mathrm{L}} \text { TALK. SOLDIER SUMMER PARTY ORGANIZE. }\end{array}$ \\
\hline F20s & subject & $\begin{array}{l}\text { SOLDAT IX } \mathbf{R}_{\mathbf{R}} \text { PILOT_ _ UNTERHALT. SOLDAT SOMMERPARTY ORGANISIER. } \\
\text { SOLDIER IX }{ }_{\mathrm{R}} \text { PILOT_ }\end{array}$ \\
\hline $\mathrm{F} 20 \mathrm{o}$ & object & $\begin{array}{l}\text { SOLDAT__ PILOT IX } \mathbf{X}_{\mathrm{L}} \text { UNTERHALT. SOLDAT SOMMERPARTY ORGANISIER. } \\
\text { SOLDIER _ PILOT IX }{ }_{\text {L }} \text { TALK. SOLDIER SUMMER PARTY ORGANIZE. }\end{array}$ \\
\hline F20n & none & $\begin{array}{l}\text { SOLDAT _ PILOT _ UNTERHALT. SOLDAT SOMMERPARTY ORGANISIER. } \\
\text { SOLDIER _ PILOT_ TALK. SOLDIER SUMMER PARTY ORGANIZE. }\end{array}$ \\
\hline F21so & both & $\begin{array}{l}\text { CLOWN IX } \mathbf{R}_{\mathbf{R}} \text { MÄDCHEN IX } \mathbf{X}_{\mathbf{L}} \text { DISKUTIER. CLOWN BESSER RECHNEN KANN. } \\
\text { CLOWN IX } \mathrm{X}_{\mathrm{R}} \text { GIRL IX } \mathrm{I}_{\mathrm{L}} \text { DISCUSS. CLOWN BETTER CALCULATE CAN. }\end{array}$ \\
\hline $\mathrm{F} 21 \mathrm{~s}$ & subject & 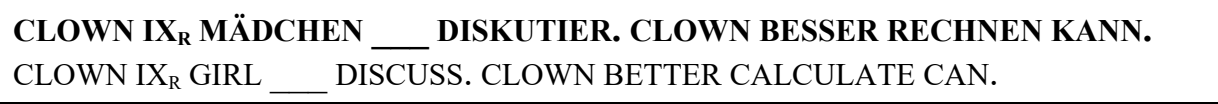 \\
\hline F21o & object & 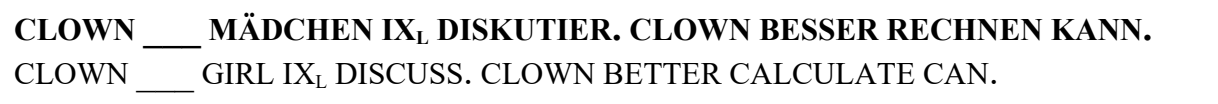 \\
\hline F21n & none & $\begin{array}{l}\text { CLOWN _ MÄDCHEN _ DISKUTIER. CLOWN BESSER RECHNEN KANN. } \\
\text { CLOWN__ GIRL__ DISCUSS. CLOWN BETTER CALCULATE CAN. }\end{array}$ \\
\hline F22so & both & $\begin{array}{l}\text { JÄGER IX }_{\mathbf{R}} \text { POLIZIST IX } \mathbf{X}_{\mathbf{L}} \text { DISKUTIER. JÄGERN FUBBALLSPIEL ORGANISIER. } \\
\text { HUNTER IX }{ }_{R} \text { POLICEMAN IX }{ }_{\text {L }} \text { DISCUSS. HUNTER SOCCER GAME ORGANIZE. }\end{array}$ \\
\hline
\end{tabular}




\begin{tabular}{|c|c|c|}
\hline F $22 s$ & subject & $\begin{array}{l}\begin{array}{l}\text { JÄGER IX }_{\mathbf{R}} \text { POLIZIST } \\
\text { HUNTER IX }\end{array} \text { ROLICEMAN_DISKUTIER. JÄGERN FUBBALLSPIEL ORGANISIER. } \\
\text { DISCUSS. HUNTER SOCCER GAME ORGANIZE. }\end{array}$ \\
\hline F22o & object & 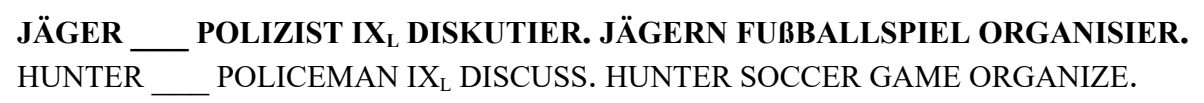 \\
\hline F22n & none & 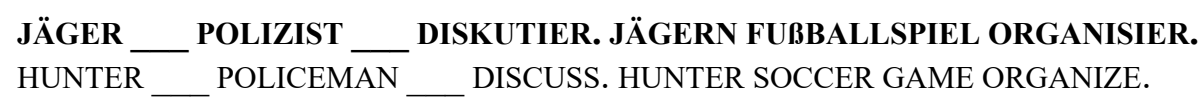 \\
\hline F23so & both & $\begin{array}{l}\text { DETEKTIV IX }_{R} \text { ARZT IX }{ }_{L} \text { DISKUTIER. DETEKTIV TERMIN NEU BRAUCH. } \\
\text { DETECTIVE IX } X_{R} \text { DOCTOR IX } X_{L} \text { DISCUSS. DETECTIVE APPOINTMENT NEW NEED. }\end{array}$ \\
\hline $\mathrm{F} 23 \mathrm{~s}$ & subject & \begin{tabular}{|l} 
DETEKTIV IX $_{\text {R ARZT }} \_$DISKUTIER. DETEKTIV TERMIN NEU BRAUCH. \\
DETECTIVE IX $_{R}$ DOCTOR__ DISCUSS. DETECTIVE APPOINTMENT NEW NEED.
\end{tabular} \\
\hline F23o & object & 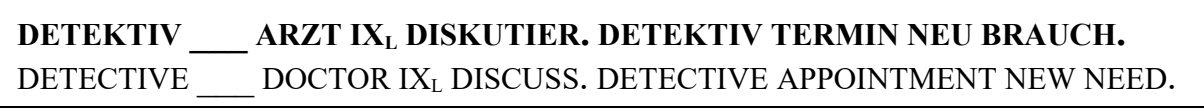 \\
\hline F23n & none & 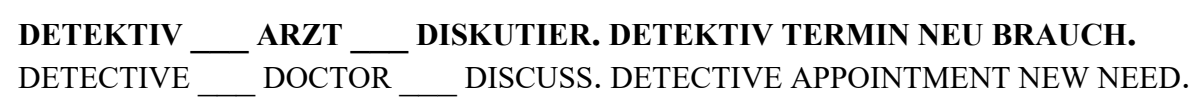 \\
\hline F24so & both & $\begin{array}{l}\text { KELLNER IX } \mathbf{R}_{\mathbf{R}} \text { FRAU IX } \mathbf{X}_{\mathbf{L}} \text { DISKUTIER. KELLNER MEHR ARBEIT MUSS. } \\
\text { WAITER IX }_{R} \text { WOMAN IX } \\
\text { LISCUSS. WAITER MORE WORK HAVE-TO. }\end{array}$ \\
\hline $\mathrm{F} 24 \mathrm{~s}$ & subject & $\begin{array}{l}\text { KELLNER IX } \mathbf{X}_{\mathbf{R}} \text { FRAU — DISKUTIER. KELLNER MEHR ARBEIT MUSS. } \\
\text { WAITER IX } \mathrm{X}_{\mathrm{R}} \text { WOMAN } \\
\text { DISCUSS. WAITER MORE WORK HAVE-TO. }\end{array}$ \\
\hline F24o & object & $\begin{array}{l}\text { KELLNER _ } \text { FRAU IX }_{\text {L }} \text { DISKUTIER. KELLNER MEHR ARBEIT MUSS. } \\
\text { WAITER _ WOMAN IX } \mathrm{X}_{\text {L }} \text { DISCUSS. WAITER MORE WORK HAVE-TO. }\end{array}$ \\
\hline F24n & none & $\begin{array}{l}\text { KELLNER _ FRAU _ DISKUTIER. KELLNER MEHR ARBEIT MUSS. } \\
\text { WAITER_WOMAN _ DISCUSS. WAITER MORE WORK HAVE-TO. }\end{array}$ \\
\hline F25so & both & $\begin{array}{l}\text { PIRAT IX } \mathbf{X}_{\mathbf{R}} \text { PRIESTER IX } \mathbf{X}_{\mathbf{L}} \text { BEGEG. PIRAT KLEIDUNG WASCHEN MUSS. } \\
\text { PIRATE IX }_{R} \text { PRIEST IX } \mathrm{X}_{L} \text { ENCOUNTER. PIRATE CLOTHES WASH HAVE-TO. }\end{array}$ \\
\hline $\mathrm{F} 25 \mathrm{~s}$ & subject & 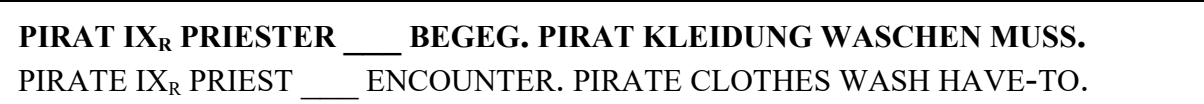 \\
\hline $\mathrm{F} 25 \mathrm{o}$ & object & $\begin{array}{l}\text { PIRAT___ PRIESTER IX } \text { L }_{\text {LEGEG. PIRAT KLEIDUNG WASCHEN MUSS. }} \\
\text { PIRATE__PRIEST IX } X_{L} \text { ENCOUNTER. PIRATE CLOTHES WASH HAVE-TO. }\end{array}$ \\
\hline F25n & none & $\begin{array}{l}\text { PIRAT__ PRIESTER } \\
\text { PIRATE__ PRIEST } \_ \text {ENCOUNTER. PIRATE CLOTHES WASH HAVE-TO. }\end{array}$ \\
\hline F26so & both & $\begin{array}{l}\text { HEXE IX } \mathbf{X}_{\mathrm{R}} \text { KÖNIG IX }{ }_{\mathrm{L}} \text { BEGEG. HEXE KIRCHE ALT BESICHTIG. } \\
\text { WITCH IX } \mathrm{X}_{\mathrm{R}} \text { KING IX } \mathrm{X}_{\mathrm{L}} \text { ENCOUNTER. WITCH CHURCH OLD VISIT. } \\
\end{array}$ \\
\hline F26s & subject & $\begin{array}{l}\text { HEXE IX } \mathbf{X}_{\mathbf{R}} \text { KÖNIG — BEGEG. HEXE KIRCHE ALT BESICHTIG. } \\
\text { WITCH IX } X_{R} \text { KING_ ENCOUNTER. WITCH CHURCH OLD VISIT. }\end{array}$ \\
\hline F26o & object & 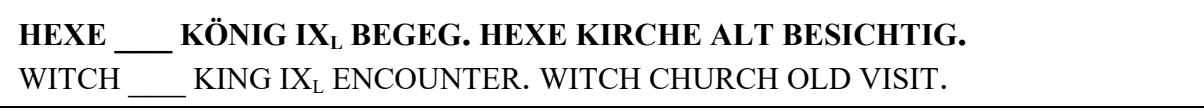 \\
\hline F26n & none & $\begin{array}{l}\text { HEXE__ KÖNIG__ BEGEG. HEXE KIRCHE ALT BESICHTIG. } \\
\text { WITCH_KING__ENCOUNTER. WITCH CHURCH OLD VISIT. }\end{array}$ \\
\hline F27so & both & $\begin{array}{l}\text { NONNE IX } \mathbf{X}_{\mathrm{R}} \text { KELLNER IX } \mathbf{X}_{\mathrm{L}} \text { BEGEG. NONNE SCHIRM ROT VERGESS. } \\
\text { NUN IX } \mathrm{X}_{\mathrm{R}} \text { WAITER IX } \mathrm{X}_{\mathrm{L}} \text { ENCOUNTER. NUN UMBRELLA RED FORGET. }\end{array}$ \\
\hline $\mathrm{F} 27 \mathrm{~s}$ & subject & $\begin{array}{l}\text { NONNE IX } \mathbf{X}_{\mathbf{R}} \text { KELLNER _ BEGEG. NONNE SCHIRM ROT VERGESS. } \\
\text { NUN IX }{ }_{R} \text { WAITER __ENCOUNTER. NUN UMBRELLA RED FORGET. }\end{array}$ \\
\hline $\mathrm{F} 27 \mathrm{o}$ & object & $\begin{array}{l}\text { NONNE } \_ \text {KELLNER IX } \mathbf{X}_{\mathbf{L}} \text { BEGEG. NONNE SCHIRM ROT VERGESS. } \\
\text { NUN _ WAITER IX }{ }_{L} \text { ENCOUNTER. NUN UMBRELLA RED FORGET. }\end{array}$ \\
\hline $\mathrm{F} 27 \mathrm{n}$ & none & $\begin{array}{l}\text { NONNE } \\
\text { NUN_ KELLNER _ WAITER__ BNCOUNTER. NUN UMBRELLA RED FORGET. }\end{array}$ \\
\hline
\end{tabular}




\begin{tabular}{|c|c|c|}
\hline F28so & both & $\begin{array}{l}\text { LEHRER IX } \mathbf{X}_{\mathbf{R}} \text { SOLDAT IX } \mathbf{X}_{\mathbf{L}} \text { BEGEG. LEHRER WEGBESCHREIBUNG BRAUCH. } \\
\text { TEACHER IX }_{\mathrm{R}} \text { SOLDIER IX }{ }_{\mathrm{R}} \text { ENCOUNTER. TEACHER WAY DIRECTION NEED. }\end{array}$ \\
\hline $\mathrm{F} 28 \mathrm{~s}$ & subject & $\begin{array}{l}\text { LEHRER IX } \mathbf{X}_{\mathbf{R}} \text { SOLDAT — BEGEG. LEHRER WEGBESCHREIBUNG BRAUCH. } \\
\text { TEACHER IX }_{\mathrm{R}} \text { SOLDIER — ENCOUNTER. TEACHER WAY DIRECTION NEED. }\end{array}$ \\
\hline F28o & object & $\begin{array}{l}\text { LEHRER__ SOLDAT IX } \mathbf{X}_{\mathbf{L}} \text { BEGEG. LEHRER WEGBESCHREIBUNG BRAUCH. } \\
\text { TEACHER__ SOLDIER IX } X_{R} \text { ENCOUNTER. TEACHER WAY DIRECTION NEED. }\end{array}$ \\
\hline $\mathrm{F} 28 \mathrm{n}$ & none & $\begin{array}{l}\text { LEHRER__ SOLDAT__ BEGEG. LEHRER WEGBESCHREIBUNG BRAUCH. } \\
\text { TEACHER__ SOLDIER__ ENCOUNTER. TEACHER WAY DIRECTION NEED. }\end{array}$ \\
\hline F29so & both & 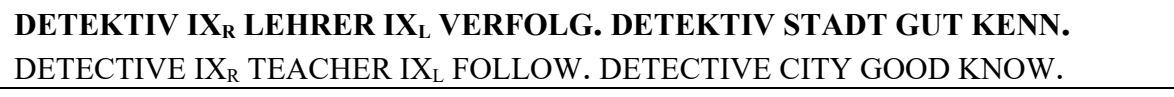 \\
\hline F29s & subject & $\begin{array}{l}\text { DETEKTIV IX } \text { R }_{\mathbf{R}} \text { LEHRER__ VERFOLG. DETEKTIV STADT GUT KENN. } \\
\text { DETECTIVE IX }_{\text {R TEACHER _ _ FOLLOW. DETECTIVE CITY GOOD KNOW. }}\end{array}$ \\
\hline F29o & object & $\begin{array}{l}\text { DETEKTIV _ LEHRER IX } \mathbf{X}_{\text {L VERFOLG. DETEKTIV STADT GUT KENN. }} \text { LETECTIVE_ TEACHER IX }{ }_{\text {L }} \text { FOLLOW. DETECTIVE CITY GOOD KNOW. } \\
\text { DET }\end{array}$ \\
\hline F29n & none & $\begin{array}{l}\text { DETEKTIV__ LEHRER__ VERFOLG. DETEKTIV STADT GUT KENN. } \\
\text { DETECTIVE_ TEACHER_ FOLLOW. DETECTIVE CITY GOOD KNOW. }\end{array}$ \\
\hline F30so & both & 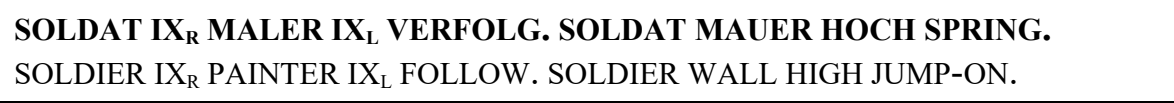 \\
\hline F30s & subject & $\begin{array}{l}\text { SOLDAT IX } \mathbf{X}_{\mathbf{R}} \text { MALER__ VERFOLG. SOLDAT MAUER HOCH SPRING. } \\
\text { SOLDIER IX }_{\mathrm{R}} \text { PAINTER__ FOLLOW. SOLDIER WALL HIGH JUMP-ON. }\end{array}$ \\
\hline F30o & object & $\begin{array}{l}\text { SOLDAT _ MALER IX } \mathbf{X}_{\mathbf{L}} \text { VERFOLG. SOLDAT MAUER HOCH SPRING. } \\
\text { SOLDIER _ PAINTER IX } X_{\text {L }} \text { FOLLOW. SOLDIER WALL HIGH JUMP-ON. }\end{array}$ \\
\hline F30n & none & $\begin{array}{l}\text { SOLDAT_ MALER__ VERFOLG. SOLDAT MAUER HOCH SPRING. } \\
\text { SOLDIER__ PAINTER _ FOLLOW. SOLDIER WALL HIGH JUMP-ON. }\end{array}$ \\
\hline F31so & both & $\begin{array}{l}\text { OPA IX } \mathbf{X}_{\mathbf{R}} \text { PRIESTER IX } \mathbf{X}_{\mathbf{L}} \text { VERFOLG. OPA FLUSS TIEF (REIN)FALL. } \\
\text { GRANDPA IX } \mathrm{X}_{\mathrm{R}} \text { PRIEST IX } \mathrm{I}_{\mathrm{L}} \text { FOLLOW. GRANDPA RIVER DEEP FALL-INTO. }\end{array}$ \\
\hline F31s & subject & $\begin{array}{l}\text { OPA IX }_{\mathbf{R}} \text { PRIESTER __ VERFOLG. OPA FLUSS TIEF (REIN)FALL. } \\
\text { GRANDPA IX }_{R} \text { PRIEST _ FOLLOW. GRANDPA RIVER DEEP FALL-INTO. }\end{array}$ \\
\hline F31o & object & 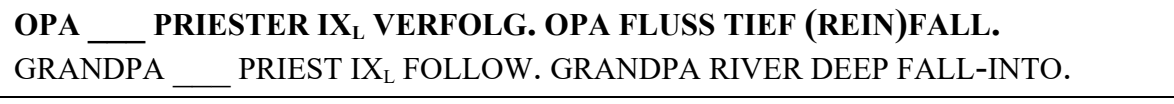 \\
\hline F31n & none & $\begin{array}{l}\text { OPA___PRIESTER __ VERFOLG. OPA FLUSS TIEF (REIN)FALL. } \\
\text { GRANDPA__PRIEST__ FOLLOW. GRANDPA RIVER DEEP FALL-INTO. }\end{array}$ \\
\hline F32so & both & $\begin{array}{l}\text { ARZT IX } \mathbf{X}_{\mathbf{R}} \text { KOCH IX } \mathbf{X}_{\mathbf{L}} \text { VERFOLG. ARZT BANANE MATSCHIG AUSRUTSCH. } \\
\text { DOCTOR IX }{ }_{\mathrm{R}} \text { COOK IX } \mathrm{X}_{\mathrm{L}} \text { FOLLOW. DOCTOR BANANA MUDDY SLIP. }\end{array}$ \\
\hline F32s & subject & 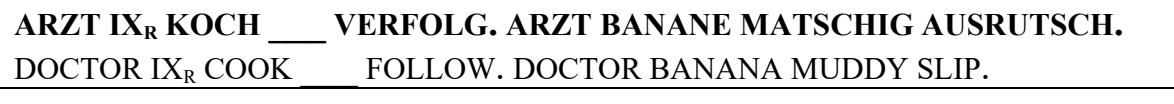 \\
\hline F32o & object & $\begin{array}{l}\text { ARZT _ KOCH IX } \mathbf{X}_{\mathbf{L}} \text { VERFOLG. ARZT BANANE MATSCHIG AUSRUTSCH. } \\
\text { DOCTOR _ COOK IX } \mathrm{L}_{\mathrm{L}} \text { FOLLOW. DOCTOR BANANA MUDDY SLIP. }\end{array}$ \\
\hline F32n & none & $\begin{array}{l}\text { ARZT _ KOCH _ VERFOLG. ARZT BANANE MATSCHIG AUSRUTSCH. } \\
\text { DOCTOR_ COOK } \_ \text {FOLLOW. DOCTOR BANANA MUDDY SLIP. }\end{array}$ \\
\hline F33so & both & $\begin{array}{l}\text { DIEB IX } \mathbf{X}_{\mathbf{R}} \text { OMA IX } \mathbf{X}_{\mathbf{L}} \text { STREIT. DIEB INNERLICH GROßE ANGST. } \\
\text { THIEF IX }_{R} \text { GRANDMA IX } \mathrm{X}_{\mathrm{L}} \text { ARGUE. THIEF INTERNAL A-LOT FEAR. }\end{array}$ \\
\hline F33s & subject & $\begin{array}{l}\text { DIEB IX } \mathbf{X}_{\mathbf{R}} \text { OMA _ STREIT. DIEB INNERLICH GROßE ANGST. } \\
\text { THIEF IX }_{R} \text { GRANDMA__ ARGUE. THIEF INTERNAL A-LOT FEAR. }\end{array}$ \\
\hline F33o & object & $\begin{array}{l}\text { DIEB __ OMA IX } \text { L }_{\mathbf{L}} \text { STREIT. DIEB INNERLICH GROßE ANGST. } \\
\text { THIEF_ GRANDMA IX ARGUE. THIEF INTERNAL A-LOT FEAR. }\end{array}$ \\
\hline
\end{tabular}




\begin{tabular}{|c|c|c|}
\hline F33n & none & $\begin{array}{l}\text { DIEB __ OMA _ _ STREIT. DIEB INNERLICH GROßE ANGST. } \\
\text { THIEF__ GRANDMA__ ARGUE. THIEF INTERNAL A-LOT FEAR. }\end{array}$ \\
\hline F34so & both & $\begin{array}{l}\text { PRIESTER IX } X_{\text {R }} \text { ARZT IX } \text { I }_{L} \text { STREIT. PRIESTER KISTE SCHWER WERF. } \\
\text { PRIEST IX } X_{R} \text { DOCTOR IX }{ }_{L} \text { ARGUE. PRIEST BOX HEAVY THROW. }\end{array}$ \\
\hline F34s & subject & 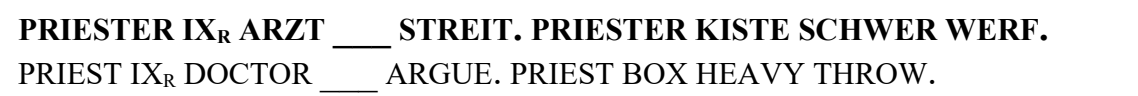 \\
\hline F34o & object & 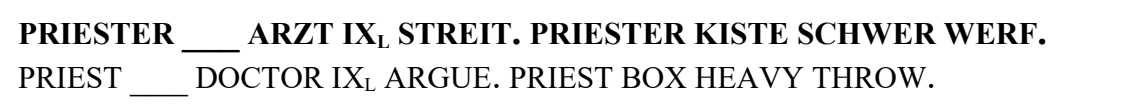 \\
\hline F34n & none & $\begin{array}{l}\text { PRIESTER _ ARZT _ STREIT. PRIESTER KISTE SCHWER WERF. } \\
\text { PRIEST _ DOCTOR _ ARGUE. PRIEST BOX HEAVY THROW. }\end{array}$ \\
\hline F35so & both & $\begin{array}{l}\text { PILOT IX } \mathbf{X}_{\mathbf{R}} \text { FRAU IX } \mathbf{X}_{\mathrm{L}} \text { STREIT. PILOT WORT SCHLIMM BENUTZ. } \\
\text { PILOT IX } \text { WOMAN IX } \\
\end{array}$ \\
\hline F35s & subject & 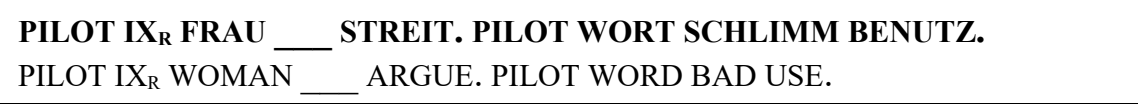 \\
\hline F35o & object & $\begin{array}{l}\text { PILOT__ FRAU IX } \text { I }_{\text {L }} \text { STREIT. PILOT WORT SCHLIMM BENUTZ. } \\
\text { PILOT_ WOMAN IX } \text { L }_{\text {L ARGUE. PILOT WORD BAD USE. }}\end{array}$ \\
\hline F35n & none & $\begin{array}{l}\text { PILOT _ FRAU _ _ STREIT. PILOT WORT SCHLIMM BENUTZ. } \\
\text { PILOT_ WOMAN__ ARGUE. PILOT WORD BAD USE. }\end{array}$ \\
\hline F36so & both & $\begin{array}{l}\text { INDIANER IX } \mathbf{R}_{\mathbf{R}} \text { MANN IX } \mathbf{X}_{\mathbf{L}} \text { STREIT. INDIANER ALKOHOL HART TRINK. } \\
\text { AMERINDIAN IX } \mathrm{X}_{\mathrm{R}} \text { MAN IX } \mathrm{X}_{\mathrm{L}} \text { ARGUE. AMERINDIAN ALCOHOL HARD DRINK. }\end{array}$ \\
\hline F36s & subject & 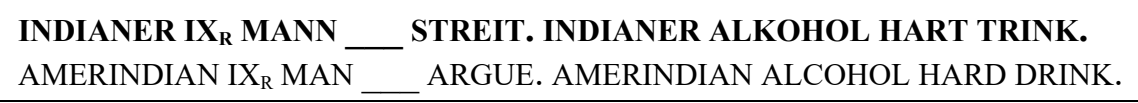 \\
\hline F36o & object & $\begin{array}{l}\text { INDIANER _ MANN IX } \text { X }_{\mathrm{L}} \text { STREIT. INDIANER ALKOHOL HART TRINK. } \\
\text { AMERINDIAN _ MAN IX } \mathrm{L}_{\mathrm{L}} \text { ARGUE. AMERINDIAN ALCOHOL HARD DRINK. }\end{array}$ \\
\hline F36n & none & $\begin{array}{l}\text { INDIANER _ MANN__ STREIT. INDIANER ALKOHOL HART TRINK. } \\
\text { AMERINDIAN _ MAN__ ARGUE. AMERINDIAN ALCOHOL HARD DRINK. }\end{array}$ \\
\hline F37so & both & $\begin{array}{l}\text { KOCH IX } \mathbf{X}_{\mathbf{R}} \text { CLOWN IX } \\
\text { L PRÜGEL. KOCH HASS STARK FÜHL. } \\
\text { COOK IX }{ }_{R} \text { CLOWN IX } \\
\text { LEAT. COOK HATE STRONG FEEL. }\end{array}$ \\
\hline F37s & subject & $\begin{array}{l}\text { KOCH IX } \mathbf{X}_{\mathbf{R}} \text { CLOWN _ PRÜGEL. KOCH HASS STARK FÜHL. } \\
\text { COOK IX } \mathrm{X}_{\mathrm{R}} \text { CLOWN_ BEAT. COOK HATE STRONG FEEL. }\end{array}$ \\
\hline F37o & object & $\begin{array}{l}\text { KOCH _ CLOWN IX } \mathbf{X}_{\mathbf{L}} \text { PRÜGEL. KOCH HASS STARK FÜHL. } \\
\text { COOK__ CLOWN IX BEAT. COOK HATE STRONG FEEL. }\end{array}$ \\
\hline F37n & none & $\begin{array}{l}\text { KOCH _ CLOWN _ PRÜGEL. KOCH HASS STARK FÜHL. } \\
\text { COOK__LOWN_ BEAT. COOK HATE STRONG FEEL. }\end{array}$ \\
\hline F38so & both & $\begin{array}{l}\text { PILOT IX } \mathbf{X}_{\mathbf{R}} \text { KELLNER IX } \mathbf{X}_{\text {L }} \text { PRÜGEL. PILOT TELLER SCHÖN ZERBRECH. } \\
\text { PILOT IX } \mathrm{X}_{\mathrm{R}} \text { WAITER IX } \mathrm{X}_{\mathrm{L}} \text { BEAT. PILOT PLATE NICE CRACK. }\end{array}$ \\
\hline F38s & subject & $\begin{array}{l}\text { PILOT IX }_{\mathbf{R}} \text { KELLNER } \\
\text { PILOT IX }{ }_{\mathrm{R}} \text { WAITER } \\
\end{array}$ \\
\hline F38o & object & $\begin{array}{l}\text { PILOT__ KELLNER IX } \mathbf{X}_{\text {L }} \text { PRÜGEL. PILOT TELLER SCHÖN ZERBRECH. } \\
\text { PILOT_ WAITER IX } X_{\text {L BEAT. PILOT PLATE NICE CRACK. }}\end{array}$ \\
\hline F38n & none & $\begin{array}{l}\text { PILOT _ KELLNER _ PRÜGEL. PILOT TELLER SCHÖN ZERBRECH. } \\
\text { PILOT _ WAITER BEAT. PILOT PLATE NICE CRACK. }\end{array}$ \\
\hline F39so & both & $\begin{array}{l}\text { ARZT IX }_{\mathbf{R}} \text { LEHRER IX } \mathbf{L}_{\mathrm{L}} \text { PRÜGEL. ARZT TREPPE LANG HERUNTER-ROLL. } \\
\text { DOCTOR IX } \mathrm{X}_{\mathrm{R}} \text { TEACHER IX } \mathrm{X}_{\mathrm{L}} \text { BEAT. DOCTOR STAIRS LONG TUMBLE-DOWN. }\end{array}$ \\
\hline F39s & subject & $\begin{array}{l}\text { ARZT IX }_{\text {R LEHRER __ PRÜGEL. ARZT TREPPE LANG HERUNTER-ROLL. }} \text { P } \\
\begin{array}{l}\text { DOCTOR IX } \\
\text { REACHER }\end{array} \text { BEAT. DOCTOR STAIRS LONG TUMBLE-DOWN. }\end{array}$ \\
\hline
\end{tabular}




\begin{tabular}{|c|c|c|}
\hline F39o & object & $\begin{array}{l}\text { ARZT__ LEHRER IX } \mathbf{X}_{\mathbf{L}} \text { PRÜGEL. ARZT TREPPE LANG HERUNTER-ROLL. } \\
\text { DOCTOR _ TEACHER IX }{ }_{\text {L }} \text { BEAT. DOCTOR STAIRS LONG TUMBLE-DOWN. }\end{array}$ \\
\hline F39n & none & $\begin{array}{l}\text { ARZT _ _ LEHRER__ PRÜGEL. ARZT TREPPE LANG HERUNTER-ROLL. } \\
\text { DOCTOR _ TEACHER _ BEAT. DOCTOR STAIRS LONG TUMBLE-DOWN. }\end{array}$ \\
\hline F40so & both & $\begin{array}{l}\text { MALER IX } \mathbf{X}_{\mathbf{R}} \text { MANN IX } \mathbf{X}_{\mathbf{L}} \text { PRÜGEL. MALER DREI ZÄHNE VERLIER. } \\
\text { PAINTER IX }{ }_{R} \text { MAN IX }{ }_{L} \text { BEAT. PAINTER THREE TEETH LOOSE. }\end{array}$ \\
\hline $\mathrm{F} 40 \mathrm{~s}$ & subject & $\begin{array}{l}\text { MALER IX }_{\mathbf{R}} \text { MANN___ PRÜGEL. MALER DREI ZÄHNE VERLIER. } \\
\text { PAINTER IX }{ }_{\text {R }} \text { MAN__ BEAT. PAINTER THREE TEETH LOOSE. }\end{array}$ \\
\hline F40o & object & $\begin{array}{l}\text { MALER___ MANN IX } \mathbf{X}_{\mathbf{L}} \text { PRÜGEL. MALER DREI ZÄHNE VERLIER. } \\
\text { PAINTER__ MAN IX } \mathrm{X}_{\text {L }} \text { BEAT. PAINTER THREE TEETH LOOSE. }\end{array}$ \\
\hline F40n & none & $\begin{array}{l}\text { MALER___ MANN — PRÜGEL. MALER DREI ZÄHNE VERLIER. } \\
\text { PAINTER_ MAN_ BEAT. PAINTER THREE TEETH LOOSE. }\end{array}$ \\
\hline
\end{tabular}

Table 3: Stimulus material used as practice items

\begin{tabular}{|c|c|c|c|}
\hline $\begin{array}{l}\text { Video } \\
\text { name }\end{array}$ & Condition & Continuation type & Sentence set \\
\hline P01so & both & object & $\begin{array}{l}\text { ZAUBERER IX }_{\mathbf{R}} \text { GÄRTNER IX } \mathbf{X}_{\mathbf{L}} \text { BEOBACHT. } \\
\text { GÄRTNER SOFA SCHWARZ DRAUF-SITZ. } \\
\text { WIZARD IX }_{R} \text { GARDENER IX } \text { OBSERVE. GARDENER }_{\text {OBS }} \\
\text { SOFA BLACK SIT-ON. }\end{array}$ \\
\hline $\mathrm{P} 02 \mathrm{~s}$ & subject & subject & $\begin{array}{l}\text { BAUER IX } \mathbf{R}_{\mathbf{R}} \text { RICHTER —_ KENN. BAUER ANDERE } \\
\text { WOHNUNG UMZIEH. } \\
\text { FARMER IX } X_{R} \text { JUDGE —_ KNOW. FARMER ANOTHER } \\
\text { APARTMENT MOVE. }\end{array}$ \\
\hline P03n & none & subject & $\begin{array}{l}\text { BÄCKER___SEKRETÄRIN__ EINLAD. BÄCKER } \\
\text { GEBURTSTAGSPARTY VORBEREIT. } \\
\text { BAKER__ SECRETARY__ INVITE. BAKER } \\
\text { BIRTHDAY PARTY PREPARE. }\end{array}$ \\
\hline P04so & both & subject & $\begin{array}{l}\text { FEUERWEHRMANN IX }{ }_{\mathbf{R}} \text { MAZWERGNN IX } \mathbf{X}_{\mathbf{L}} \text { SUCH. } \\
\text { FEUERWEHRMANN DACH HINAUF-KLETTER. } \\
\text { FIREFIGHTER IX }{ }_{R} \text { DWARF IX } \mathrm{X}_{\mathrm{L}} \text { SEARCH. } \\
\text { FIREFIGHTER ROOF CLIMB-UP. }\end{array}$ \\
\hline P05so & both & object & $\begin{array}{l}\text { KAPITÄN IX } \mathbf{X}_{\mathbf{R}} \text { TISCHLER IX } \mathbf{X}_{\text {L ANRUF. TISCHLER }} \\
\text { BED SCHLAF. } \\
\text { CAPTAIN IX }_{R} \text { CARPENTER IX }{ }_{\text {L CALL. CARPENTER }} \\
\text { BED SLEEP. }\end{array}$ \\
\hline P06o & object & object & $\begin{array}{l}\text { ASTRONAUT } \_ \text {RITTER IX } \mathbf{X}_{\mathbf{L}} \text { GRÜß. RITTER } \\
\text { FREUNDE BESUCH. } \\
\text { ASTRONAUT __ KNIGHT IX }{ }_{\text {L GREET. KNIGHT }} \\
\text { FRIENDS VISIT. }\end{array}$ \\
\hline
\end{tabular}


Appendix F: Chapter 3 - Figure of the Elog-transformed data

The figure displays the data for object (left panel) and subject (right panel)

continuation types and each condition (orange $=$ no localization, blue $=$ object localization, green $=$ subject localization, purple $=$ localization both) for $1000 \mathrm{~ms}$ following the first fixation to one of the presented pictures.

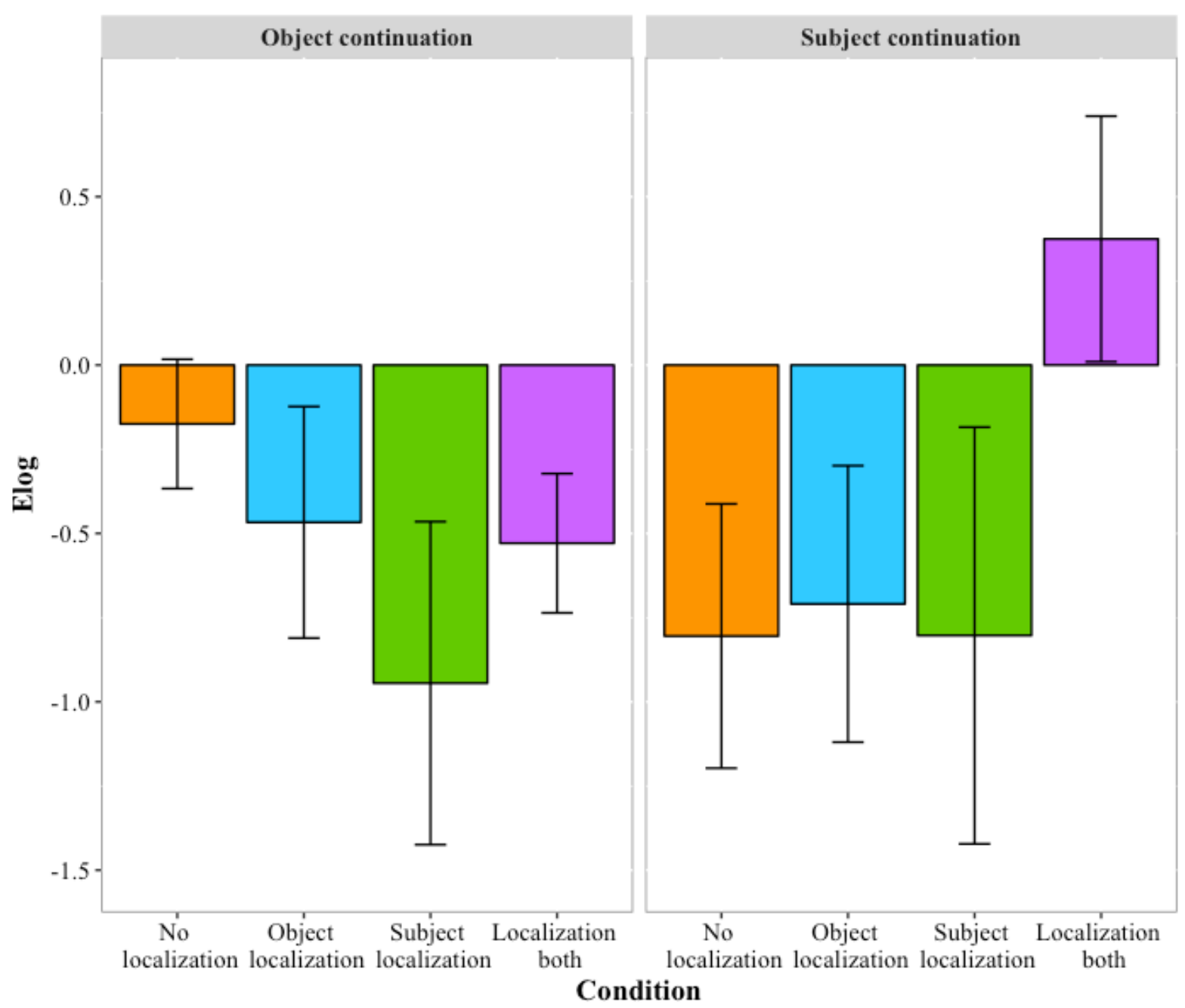


Appendix G: Chapter 4 - Complete list of stimulus material

The material for each parameter condition (denoting the differing parameter) and the practice items are presented below. Columns (from left to right) show: (i) the item number; (ii) condition ( $\mathrm{m}=$ priming; $\mathrm{o}=$ no priming); (iii) distractor; (iv) the stimulus sentence in glosses if German Sign Language with an English gloss below. Within each sentence, the bold-faced glosses specify prime (leftmost) and target (rightmost). Item 53 and 54 were excluded prior to testing due to an error in the video and are therefore marked in grey.

\begin{tabular}{|c|c|c|c|}
\hline $\begin{array}{c}\text { Item } \\
\text { ID }\end{array}$ & $\begin{array}{c}\text { Condi } \\
\text {-tion }\end{array}$ & $\begin{array}{c}\text { Distractor } \\
\text { picture }\end{array}$ & Sentence in priming and no priming condition \\
\hline & & & Sentence structure: TIME/PLACE + SUBJ $+\mathrm{CL} / \mathrm{ADJ} / \ldots+\mathrm{OBJ}+\mathrm{VERB}$ \\
\hline \multicolumn{4}{|c|}{ Parameter condition: HANDSHAPE } \\
\hline 1 & $\mathrm{~m}$ & \multirow{2}{*}{$\begin{array}{c}\text { BRILLE } \\
\text { (GLASSES) }\end{array}$} & $\begin{array}{l}\text { TÄGLICH MITTAG IX1 WASSER TRINK SOLL. } \\
\text { EVERY-DAY NOON IX1 WATER DRINK SHOULD. }\end{array}$ \\
\hline 2 & o & & $\begin{array}{l}\text { GESTERN ARBEIT IX1 WASSER VERGESS. } \\
\text { YESTERDAY WORK IX1 WATER FORGET. }\end{array}$ \\
\hline 3 & $\mathrm{~m}$ & \multirow{2}{*}{$\begin{array}{l}\text { SCHMETTERLING } \\
\text { (BUTTERFLY) }\end{array}$} & $\begin{array}{l}\text { NÄCHSTE WOCHE RAUM IX FEIER GIB. } \\
\text { NEXT WEEK ROOM IX FAMILY PARTY THERE-IS. }\end{array}$ \\
\hline 4 & o & & $\begin{array}{l}\text { LETZTES JAHR WEIHNACHTEN IX FAMILIE FROH. } \\
\text { LAST YEAR CHRISTMAS IX FAMILY HAPPY. }\end{array}$ \\
\hline 5 & $\mathrm{~m}$ & \multirow{2}{*}{$\begin{array}{l}\text { APFEL } \\
\text { (APPLE) }\end{array}$} & $\begin{array}{l}\text { IX1 URLAUB LONDON IX TOILETTE SUCH. } \\
\text { IX1 HOLIDAY LONDON IX TOILET SEARCH. }\end{array}$ \\
\hline 6 & o & & $\begin{array}{l}\text { IX1 KONFERENT BESUCH IX TOILETTE FIND } \\
\text { IX1 CONFERENCE VISIT IX TOILET FIND. }\end{array}$ \\
\hline 7 & $\mathrm{~m}$ & \multirow{2}{*}{$\begin{array}{l}\text { FROSCH } \\
\text { (FROG) }\end{array}$} & $\begin{array}{l}\text { PARKPLATZ IX AUTO WIE ROBOTER AUSSEH. } \\
\text { PARKING SPOT CAR LIKE ROBOT LOOK-LIKE. }\end{array}$ \\
\hline 8 & o & & $\begin{array}{l}\text { FIRMA IX CHEF WIE ROBOTER SPRECH. } \\
\text { COMPANY IX BOSS LIKE ROBOT TALK. }\end{array}$ \\
\hline 9 & $\mathrm{~m}$ & \multirow{2}{*}{$\begin{array}{c}\text { PINSEL } \\
\text { (BRUSH) }\end{array}$} & $\begin{array}{l}\text { GESTERN IX1 MIT FERNGLAS IX EULE SUCH. } \\
\text { YESTERDAY IX1 WITH SPYGLAS IX OWL SEARCH. }\end{array}$ \\
\hline 10 & o & & $\begin{array}{l}\text { LETZE WOCHE IX MIT TELESKOP IX EULE FIND. } \\
\text { LAST WEEK IX1 WITH TELESCOPE IX OWL FIND. }\end{array}$ \\
\hline 11 & $\mathrm{~m}$ & \multirow{2}{*}{$\begin{array}{l}\text { BUCH } \\
(\mathrm{BOOK})\end{array}$} & $\begin{array}{l}\text { EBEN ZENTRUM IX STADT BESICHTIG. } \\
\text { JUST CENTRE IX CITY VISIT. }\end{array}$ \\
\hline 12 & o & & $\begin{array}{l}\text { GESTERN IX1 KRANKENHAUS IX STADT BESUCH. } \\
\text { YESTERDAY IX1 HOSPITAL IX CITY GO-TO. }\end{array}$ \\
\hline 13 & $\mathrm{~m}$ & \multirow{2}{*}{$\begin{array}{l}\text { HEMD } \\
\text { (SHIRT) }\end{array}$} & $\begin{array}{l}\text { ALLGEMEIN IX THEATER ODER ZIRKUS MAG. } \\
\text { IN-GENERAL IX THEATER OR CIRCUS LIKE. }\end{array}$ \\
\hline 14 & o & & $\begin{array}{l}\text { FRÜHER IX1 KINO ODER ZIRKUS BESUCH. } \\
\text { BEFORE IX1 CINEMA OR CIRCUS VISIT. } \\
\end{array}$ \\
\hline 15 & $\mathrm{~m}$ & $\begin{array}{c}\text { PILZ } \\
\text { (MUSHROOM) }\end{array}$ & $\begin{array}{l}\text { VIELE PERSONEN STRATEGIE FÜR AUTOBAHN BRAUCH. } \\
\text { A-LOT PEOPLE STRATEGY FOR HIGHWAY NEED. }\end{array}$ \\
\hline
\end{tabular}




\begin{tabular}{|c|c|c|c|}
\hline 16 & $\mathrm{o}$ & & $\begin{array}{l}\text { FAHRANFÄNGER KLAR REGEL FÜR AUTOBAHN BEFOLG MUSS. } \\
\text { NEW-DRIVER CLEAR RULE FOR HIGHWAY FOLLOW HAVE-TO. }\end{array}$ \\
\hline 17 & $\mathrm{~m}$ & \multirow{2}{*}{$\begin{array}{l}\text { PUPPE } \\
\text { (DOLL) }\end{array}$} & $\begin{array}{l}\text { DIESES JAHR KONFERENZ IX STADION DA. } \\
\text { THIS YEAR CONFERENCE IX STADIUM IS-AT. }\end{array}$ \\
\hline 18 & o & & $\begin{array}{l}\text { LETZTEN MONAT ABSTIMMUNG IX STADION GEWES. } \\
\text { LAST MONTH REFERENDUM IX STADIUM TAKE-PLACE. }\end{array}$ \\
\hline 19 & $\mathrm{~m}$ & \multirow{2}{*}{$\begin{array}{l}\text { BANANE } \\
\text { (BANANA) }\end{array}$} & $\begin{array}{l}\text { FÜR ARBEIT ARZT IX UHR DA. } \\
\text { FOR WORK DOCTOR IX WATCH HAS. }\end{array}$ \\
\hline 20 & o & & $\begin{array}{l}\text { FÜR TERMINE KRANKENSCHWESTER IX UHR WÜNSCH. } \\
\text { FOR APPOINTMENTS NURSE IX WATCH WISH. }\end{array}$ \\
\hline \multicolumn{4}{|c|}{ Parameter condition: MOVEMENT } \\
\hline 21 & $\mathrm{~m}$ & \multirow{2}{*}{$\begin{array}{l}\text { GABEL } \\
\text { (FORK) }\end{array}$} & $\begin{array}{l}\text { GESTSERN THEMA VORTRAG INHALT BAUM FÄLL. } \\
\text { YESTERDAY TOPIC PRESENTATION CONTENT TREE CUT-DOWN. }\end{array}$ \\
\hline 22 & o & & $\begin{array}{l}\text { EBEN IX1 FERNSEHER BERICHT INHALT BAUM SEH. } \\
\text { JUST IX1 TV IX REPORT CONTENT TREE SEE. }\end{array}$ \\
\hline 23 & $\mathrm{~m}$ & \multirow{2}{*}{$\begin{array}{l}\text { FLASCHE } \\
\text { (BOTTLE) }\end{array}$} & $\begin{array}{l}\text { NORMALERWEISE GESCHÄFT IX TIERE BESUCH DARF-NEG. } \\
\text { USUALLY SHOP IX ANIMALS ENTER ALLOWED-NOT. }\end{array}$ \\
\hline 24 & o & & $\begin{array}{l}\text { OFT NACHTS IX TIERE SCHLAF BRAUCH-NEG. } \\
\text { OFTEN AT-NIGHT IX ANIMALS SLEPP NEED-NOT. }\end{array}$ \\
\hline 25 & $\mathrm{~m}$ & \multirow{2}{*}{$\begin{array}{l}\text { MÜTZE } \\
\text { (CAP) }\end{array}$} & $\begin{array}{l}\text { INDIEN POSS } 3 \text { KULTUR IX BLUME WICHTIG. } \\
\text { INDIA POSS3 CULTURE IX FLOWER IMPORTANT. }\end{array}$ \\
\hline 26 & 0 & & $\begin{array}{l}\text { HOCHZEIT POSS } 3 \text { TRADITION IX BLUME BELIEBT. } \\
\text { WEDDING POSS } 3 \text { TRADITION IX FLOWER POPULAR. }\end{array}$ \\
\hline 27 & $\mathrm{~m}$ & \multirow{2}{*}{$\begin{array}{l}\text { BALL } \\
(\text { BALL) }\end{array}$} & $\begin{array}{l}\text { IX1 BALKNO SCHIRM UNTER KAFFEE TRINK. } \\
\text { IX1 BALCONY UMBRELLA UNDER COFFEE DRINK. }\end{array}$ \\
\hline 28 & o & & $\begin{array}{l}\text { IX1 GARTEN DACH UNTER KAFFEE GENIEß. } \\
\text { IX1 GARDEN ROOF UNDER COFFEE ENJOY. }\end{array}$ \\
\hline 29 & $\mathrm{~m}$ & \multirow{2}{*}{$\begin{array}{l}\text { SCHLIPS } \\
\text { (TIE) }\end{array}$} & $\begin{array}{l}\text { FAHRSCHÜLER IX VIEL ÜBUNG MIT AUTO BRAUCH. } \\
\text { STUDENT-DRIVER IX A-LOT PRACTICE WITH CAR NEED. }\end{array}$ \\
\hline 30 & o & & $\begin{array}{l}\text { BERUFSFAHRER IX VIEL ERFAHRUNG MIT AUTO DA. } \\
\text { PROFESSIONAL-DRIVER IX A-LOT EXPERINECE WITH CAR HAS. }\end{array}$ \\
\hline 31 & $\mathrm{~m}$ & \multirow{2}{*}{$\begin{array}{c}\text { BRIEF } \\
\text { (LETTER) }\end{array}$} & $\begin{array}{l}\text { GESTERN IX1 POSS1 ARBEIT IX SCHIRM VERGESS. } \\
\text { YESTERDAY IX1 POSS1 WORK IX UMBRELLA FORGET. }\end{array}$ \\
\hline 32 & o & & $\begin{array}{l}\text { OFT IX1 POSS1 INTERNAT IX SCHIRM LASS. } \\
\text { OFTEN IX1 POSS1 COLLEGE IX UMBRELLA LEAVE. }\end{array}$ \\
\hline 33 & $\mathrm{~m}$ & $\begin{array}{c}\text { SQUIRREL } \\
\text { (EIHHÖRNCHEN) }\end{array}$ & $\begin{array}{l}\text { ITALIEN STADT IX ZITRONE KAUF KANN. } \\
\text { ITALY CITY IX LEMON BUY CAN. }\end{array}$ \\
\hline 34 & o & & $\begin{array}{l}\text { NORMALERWEISE KIND IX ZITRONE ESS MAG-NEG. } \\
\text { USUALLY CHILD IX LEMON EAT LIKE-NOT. }\end{array}$ \\
\hline 35 & $\mathrm{~m}$ & \multirow{2}{*}{$\begin{array}{l}\text { KUH } \\
(\mathrm{COW})\end{array}$} & $\begin{array}{l}\text { ZEITUNG BÜRGERMEISTER INTERVIEW ÜBER ALLEE } \\
\text { VERÖFFENTLICH. } \\
\text { NEWSPAPER MAYOR INTERVIEW OVER ALLEY PUBLISH. }\end{array}$ \\
\hline 36 & o & & $\begin{array}{l}\text { INTERNET IX REPORTER FOTO ÜBER ALLEE REINSETZ. } \\
\text { INTERNET IX REPORTER PHOTO OVER ALLEY UPLOAD. }\end{array}$ \\
\hline 37 & $\mathrm{~m}$ & \multirow{2}{*}{$\begin{array}{c}\text { ZAUN } \\
(\text { FENCE })\end{array}$} & $\begin{array}{l}\text { GESTERN ABEND POSS1 FREUND BUS IX COCKTEIL TRINK. } \\
\text { YESTERDAY EVENING POSS1 FRIEND BUS IX COCKTAIL DRINK. }\end{array}$ \\
\hline 38 & 0 & & $\begin{array}{l}\text { URLAUB IX POSS1 VATER STRAND IX COCKTAIL GENIEß. } \\
\text { HOLIDAY IX POSS1 FATHER BEACH IX COCKTAIL ENJOY. }\end{array}$ \\
\hline
\end{tabular}




\begin{tabular}{|c|c|c|c|}
\hline 39 & $\mathrm{~m}$ & \multirow{2}{*}{$\begin{array}{l}\text { SCHLEIFE } \\
(\mathrm{RIBBON})\end{array}$} & $\begin{array}{l}\text { LANDSCHAFT GRÜN IX DORF WIE INSEL SCHÖN AUSSEH. } \\
\text { LANDSCAPE GREEN IX VILLAGE LIKE ISLAND NICE LOOK. }\end{array}$ \\
\hline 40 & o & & $\begin{array}{l}\text { PARKPLATZ GRAU IX BLUME WIE INSEL WIRK. } \\
\text { PARKING PLACE GREY IX FLOWER LIKE ISLAND APPEAR. }\end{array}$ \\
\hline \multicolumn{4}{|c|}{ Parameter condition: LOCATION } \\
\hline 41 & $\mathrm{~m}$ & \multirow{2}{*}{$\begin{array}{l}\text { FERNSEHER } \\
\qquad(\mathrm{TV})\end{array}$} & $\begin{array}{l}\text { ZWANZIG MINUTEN IX ARZT KOMM. } \\
\text { TWENTY MINUTES IX DOCTOR COME. }\end{array}$ \\
\hline 42 & 0 & & $\begin{array}{l}\text { TÄGLICH SEKRETÄRIN IX ARZT UNTERSTÜTZ. } \\
\text { EVERY-DAY SECRETARY IX DOCTOR SUPPORT. }\end{array}$ \\
\hline 43 & $\mathrm{~m}$ & \multirow{2}{*}{$\begin{array}{l}\text { BOOT } \\
(\text { BOAT) }\end{array}$} & $\begin{array}{l}\text { BAUERNHOF IX SCHWEIN POSS1 OMA IMMER BEIß. } \\
\text { FARM IX PIG POSS1 GRANDMA ALWAYS BITE. }\end{array}$ \\
\hline 44 & 0 & & $\begin{array}{l}\text { ZUHAUSE IX NACHBAR POSS } 1 \text { OMA IMMER ÄRGER. } \\
\text { AT-HOME IX NEIGHBOR POSS1 GRANDMA ALWAYS TEASE. }\end{array}$ \\
\hline 45 & $\mathrm{~m}$ & \multirow{2}{*}{$\begin{array}{l}\text { KATZE } \\
(\text { CAT })\end{array}$} & $\begin{array}{l}\text { THEMA GESETZ REPORTER IX RICHTER NOCHMAL FRAG. } \\
\text { TOPIC LAW REPORTER IX JUDGE AGAIN ASK. }\end{array}$ \\
\hline 46 & 0 & & $\begin{array}{l}\text { GERADE POSS1 FREUND IX RICHTER ZUSCHAU. } \\
\text { AT-THE-MOMENT POSS1 FRIEND IX JUDGE WATCH. }\end{array}$ \\
\hline 47 & $\mathrm{~m}$ & \multirow{2}{*}{$\begin{array}{l}\text { SCHLÜSSEL } \\
\quad(\text { KEY })\end{array}$} & $\begin{array}{l}\text { NORMALERWEISE KINDERDARTEN IX BIKINI KOMISCH AUFFALL. } \\
\text { USUALLY KINDERGARTEN IX BIKINI ODD ATTRACT-ATTENTION. }\end{array}$ \\
\hline 48 & 0 & & $\begin{array}{l}\text { GESTERN POSS1 SCHATZ IX BIKINI NEU KAUF. } \\
\text { YESTERDAY POSS1 DARLING IX BIKINI NEW BUY. }\end{array}$ \\
\hline 49 & $\mathrm{~m}$ & \multirow{2}{*}{$\begin{array}{l}\text { ROCK } \\
(\text { SKIRT) }\end{array}$} & $\begin{array}{l}\text { ZOO KÄFIG LEER BEDEUT IX VOGEL FLÜCHT. } \\
\text { ZOO CAGE EMPTY MEANING IX BIRD RUN-AWAY. }\end{array}$ \\
\hline 50 & 0 & & $\begin{array}{l}\text { BAUM IX NEST DA GRUND IX VOGEL BRÜT. } \\
\text { TREE IX NEST THERE-IS REASON IX BIRD HATCH. }\end{array}$ \\
\hline 51 & $\mathrm{~m}$ & \multirow{2}{*}{$\begin{array}{l}\text { RUCKSACK } \\
\text { (BACKPACK) }\end{array}$} & $\begin{array}{l}\text { VOR SONNE IX1 DUSCHE BRAUCH. } \\
\text { BEFORE SUN IX1 SHOWER NEED. }\end{array}$ \\
\hline 52 & 0 & & $\begin{array}{l}\text { NACH OPERATION IX1 DUSCHE BENUTZ DARF-NEG. } \\
\text { AFTER OPERATION IX1 SHOWER USE ALLOWED-NOT. }\end{array}$ \\
\hline 53 & $\mathrm{~m}$ & \multirow{2}{*}{$\begin{array}{l}\text { LINEAL } \\
\text { (RULER) }\end{array}$} & $\begin{array}{l}\text { KINDERGESCHICHTEN IX SCHEIN PLÖTZLICH KÖNIG VERÄNDER. } \\
\text { CHILDREN-STORIES IX PIG SUDDENLY KING TURN-INTO. }\end{array}$ \\
\hline 54 & 0 & & $\begin{array}{l}\text { REALITÄT IX BETTLER PLÖTZLICH KÖNIG KAUM PASSIER. } \\
\text { REALITY IX BEGGAR SUDDENLY KIND BARELY HAPPEN. }\end{array}$ \\
\hline 55 & $\mathrm{~m}$ & \multirow{2}{*}{$\begin{array}{c}\text { KIRCHE } \\
(\mathrm{CHURCH})\end{array}$} & $\begin{array}{l}\text { VERKEHR IX ROHR IX BUS STÖR. } \\
\text { TRAFFIC IX PIPE IX BUS DISRUPT. }\end{array}$ \\
\hline 56 & 0 & & $\begin{array}{l}\text { STRAßE IX ZEITUNG IX BUS DARÜBERFAHR. } \\
\text { STREET IX NEWSPAPER IX BUS DRIVE-OVER. }\end{array}$ \\
\hline 57 & $\mathrm{~m}$ & \multirow{2}{*}{$\begin{array}{l}\text { HOSE } \\
\text { (TROUSERS) }\end{array}$} & $\begin{array}{l}\text { KINDER IX FLAGGE MIT FISCH MAL. } \\
\text { CHILDREN IX FLAG WITH FISH PAINT. }\end{array}$ \\
\hline 58 & o & & $\begin{array}{l}\text { KUNST IX VIEL BILD MIT FISCH GIBT. } \\
\text { ART IX A-LOT PICTURE WITH FISH THERE-IS. }\end{array}$ \\
\hline 59 & $\mathrm{~m}$ & \multirow{2}{*}{$\begin{array}{l}\text { STUHL } \\
(\text { CHAIR })\end{array}$} & $\begin{array}{l}\text { ZIRKUS AUFTRITT POSS1 OMA IX CLOWN MAG-NEG. } \\
\text { CIRCUS PERFORMANCE POSS1 GRANDMA IX CLOWN LIKE-NOT }\end{array}$ \\
\hline 60 & 0 & & $\begin{array}{l}\text { GESCHICHTEN IX POSS1 TOCHTER IX CLOWN LIEB. } \\
\text { STORIES IX POSS1 DAUGHTER IX CLOWN LOVE. }\end{array}$ \\
\hline 61 & ueb01 & $\begin{array}{c}\text { SCHERE } \\
(\text { SCISSORS) }\end{array}$ & $\begin{array}{l}\text { OFT SUPERMARKT IX1 ORANGE KAUF. } \\
\text { OFTEN SUPERMARKET IX1 ORANGE BUY. }\end{array}$ \\
\hline 62 & ueb02 & $\begin{array}{l}\text { MÜLLEIMER } \\
\text { (TRASH BIN) }\end{array}$ & $\begin{array}{l}\text { GESTERN BAYERN IX HÜGEL IX FRAU STAND. } \\
\text { YEASTERDAY BAVARIA IX HILL IX WOMAN STAND. }\end{array}$ \\
\hline
\end{tabular}




\begin{tabular}{|c|c|c|l|}
\hline $\mathbf{6 3}$ & ueb03 & $\begin{array}{c}\text { NADEL } \\
\text { (NEEDLE) }\end{array}$ & $\begin{array}{l}\text { MEER IX KAPITÄN POSS3 GRAB WENN SCHIFF UNTERGEH. } \\
\text { SEA IX CAPTAIN POSS3 GRAVE IF SHIP SINK. }\end{array}$ \\
\hline $\mathbf{6 4}$ & ueb04 & $\begin{array}{c}\text { GIEßKANNE } \\
\text { (WATERING CAN) }\end{array}$ & $\begin{array}{l}\text { JEDEN TAG PARK IX HUND BALL SUCH. } \\
\text { EVERY DAY PARK IX DOG BALL SEARCH. }\end{array}$ \\
\hline $\mathbf{6 5}$ & ueb05 & $\begin{array}{c}\text { TOMATE } \\
\text { (TOMATO) }\end{array}$ & $\begin{array}{l}\text { GESTERN POSS1 MUTTER IX SHUHE NEU KAUF. } \\
\text { YESTERDAY POSS1 MOTHER IX SHOES NEW BUY. }\end{array}$ \\
\hline
\end{tabular}





\section{Selbstständigkeitserklärung}

Hiermit erkläre ich an Eides statt, dass ich die vorliegende Dissertation Processing of referential expressions in German Sign Language

selbstständig verfasst und keine anderen als die angegebenen Quellen und Hilfsmittel verwendet habe. Außerdem versichere ich, dass anderweitig keine entsprechende Promotion beantragt und die hierbei eingereichte Dissertation oder Teile daraus vorgelegt worden sind.

Des weiteren bestätige ich hiermit verbindlich, dass die digital eingereichte Version mit der schriftlichen Abhandlung übereinstimmt.

Göttingen, den 01.03.2018

Ort, Datum

Anne Wienholz 